\title{
Biological Detoxification of Enniatins
}

\author{
Dissertation \\ to obtain the $\mathrm{Ph}$. $\mathrm{D}$. degree \\ in the International Ph. D. Program for Agricultural Sciences \\ in Göttingen (IPAG) \\ at the Faculty of Agricultural Sciences, \\ Georg-August-University Göttingen, Germany
}

\author{
Presented by \\ Rosine Ghislaine Suchfort (born Fotso) \\ Born in Yaoundé (Cameroon)
}

Göttingen, September 2016 
D7

1. Name of supervisor: Prof. Dr. Petr Karlovsky

2. Name of co-supervisor: Prof. Dr. Tapani Yli-Mattila

Date of dissertation: $7^{\text {th }}$ November 2016 


\section{Contents}

\section{Chapter 1}

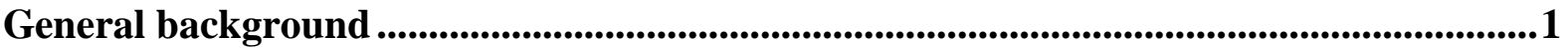

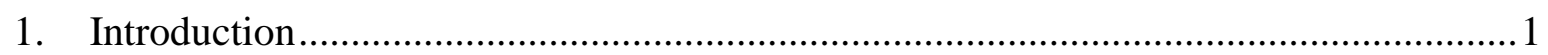

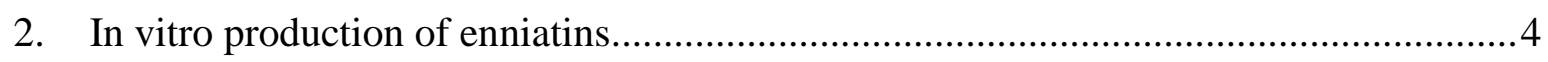

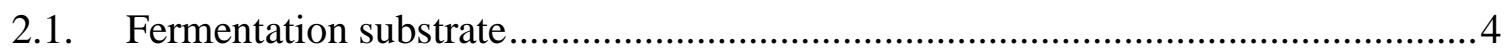

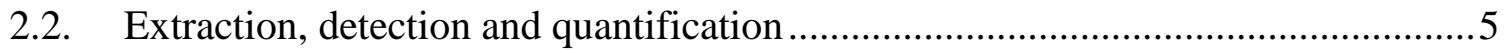

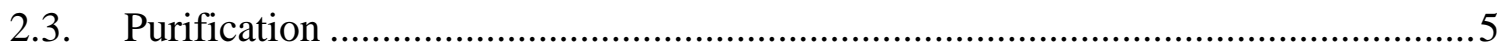

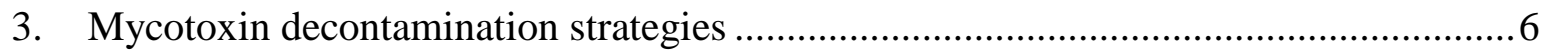

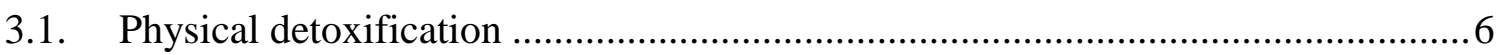

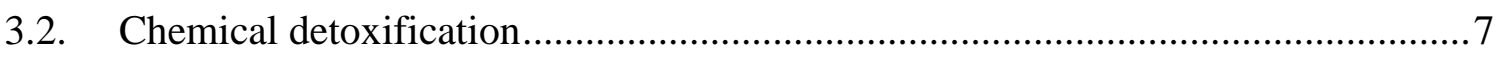

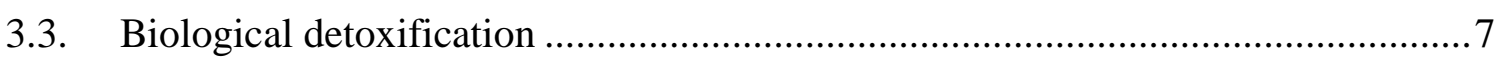

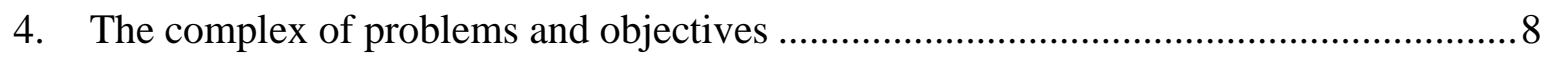

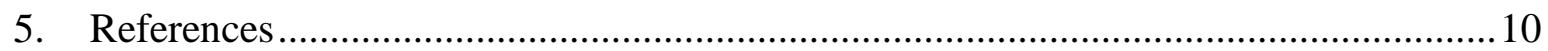

\section{Chapter 2}

Enniatins production by $F$. tricinctum on white bean medium and purification using

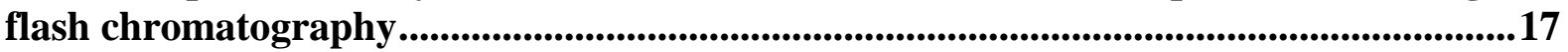

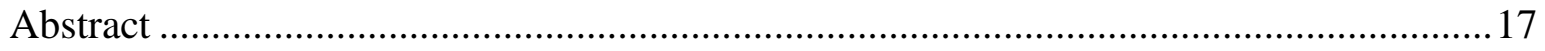

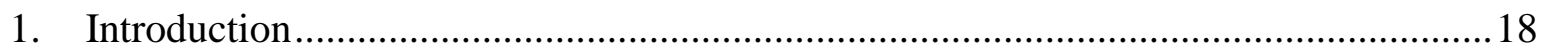

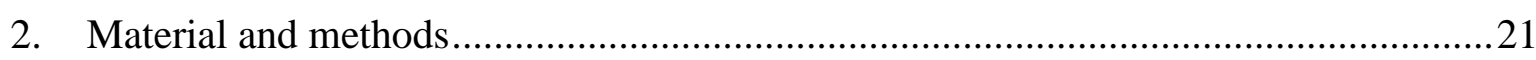

2.1. Examination of two Fusarium tricinctum strains for enniatin production ............2 21

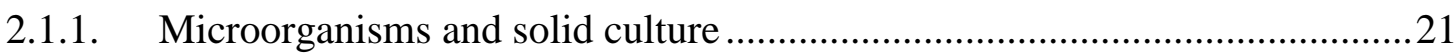

2.1.2. Extraction of enniatins and High Performance Liquid Chromatography

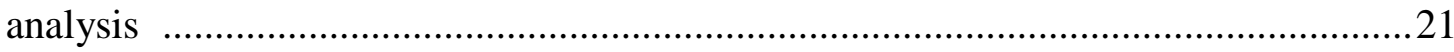

2.1.3. HPLC-MS confirmation of produced enniatins .......................................22

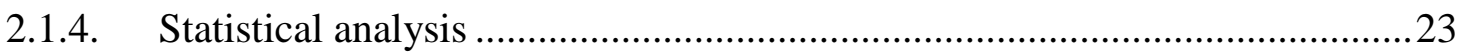

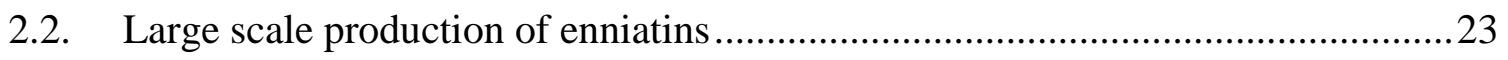

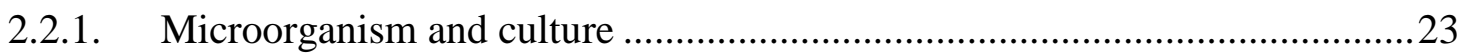

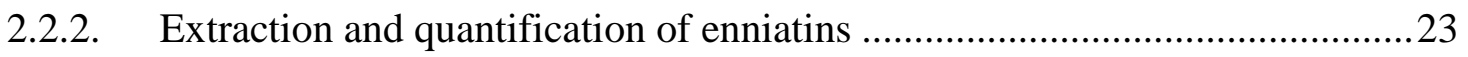

2.2.3. Preliminary purification of enniatins using flash chromatography ................24

2.2.4. Screening of flash chromatography fractions............................................... 25

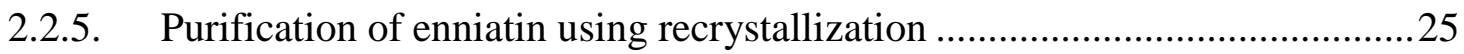

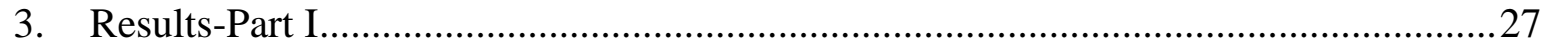

3.1. Detection of enniatin standards and crude extract using HPLC ..........................27

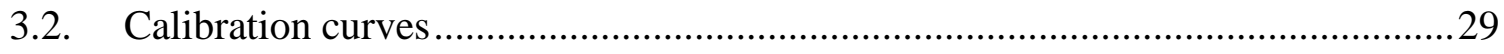

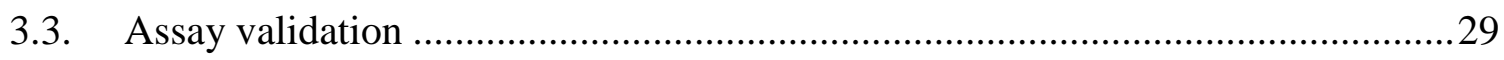


3.4. Enniatin production by $F$. tricinctum strains O32 and DSM 23357.................... 30

3.5. HPLC-MS confirmation of produced enniatins................................................. 32

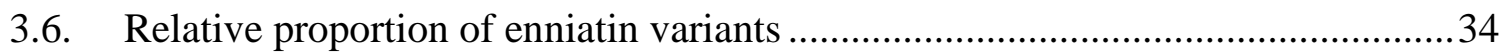

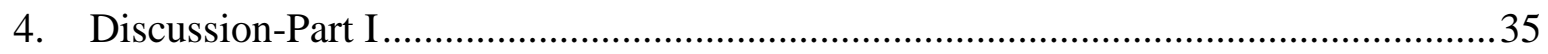

4.1. Enniatin production by F. tricinctum strains O32 and DSM 23357...................... 35

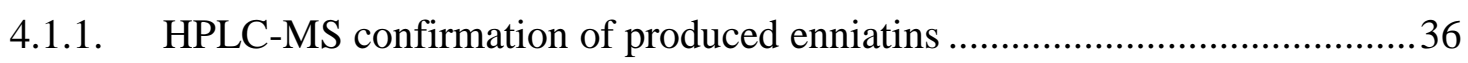

4.2. Relative proportion of enniatin variants produced by F. tricinctum $\mathrm{O} 32$ and DSM

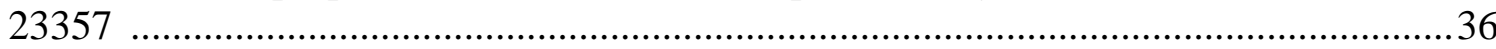

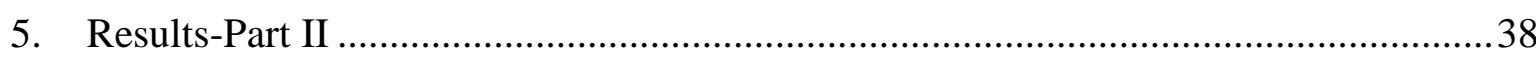

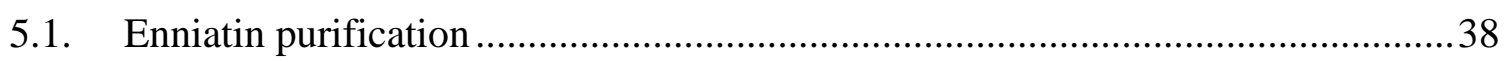

5.1.1. Screening of positive fractions after flash chromatography separation ..........38

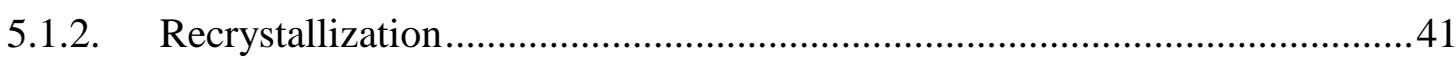

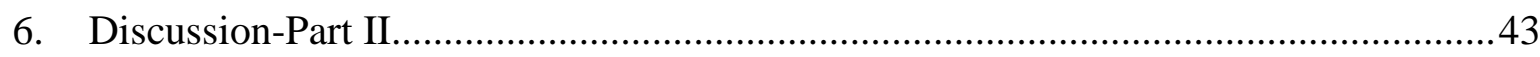

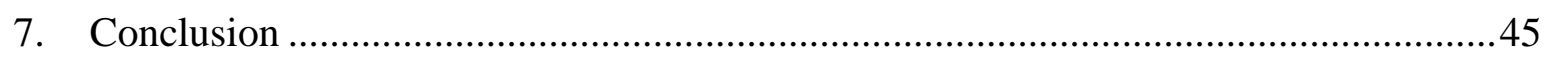

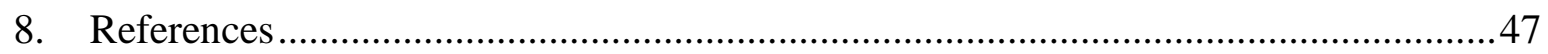

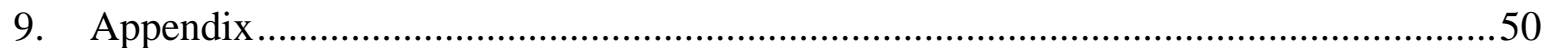

\section{Chapter 3}

Biological detoxification of enniatins and identification of degradation products using HPLC-MS

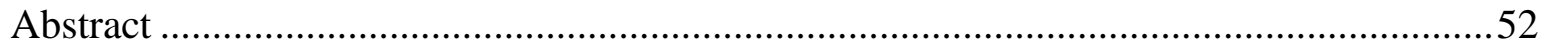

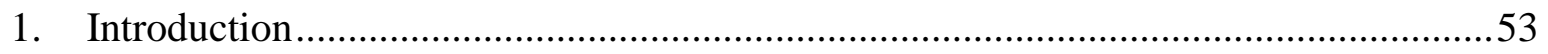

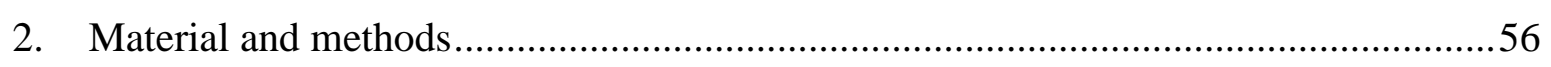

2.1. Selection of microorganisms for enniatin detoxification ...................................56

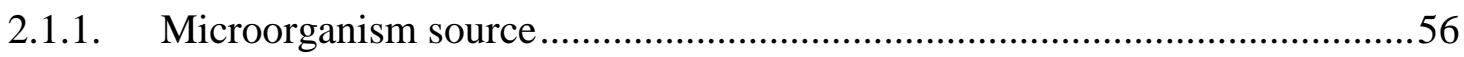

2.1.2. Selection of microorganisms from mixed culture ........................................58

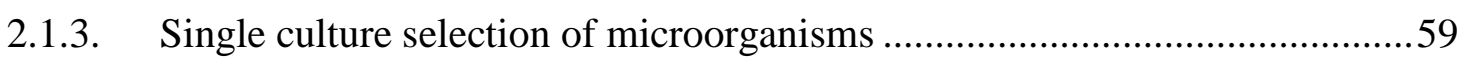

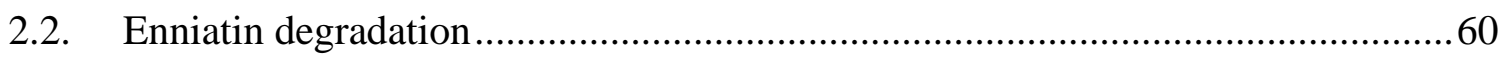

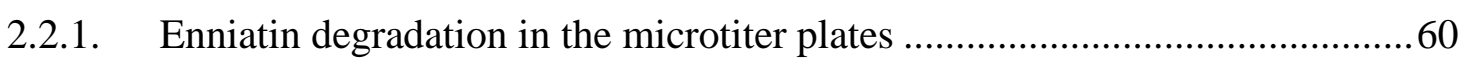

2.2.2. Bacterial and Fungal enniatin degradation under shaking conditions.............60

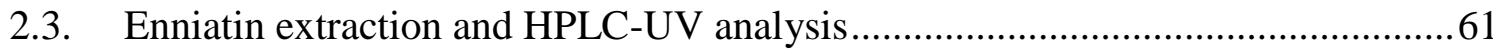

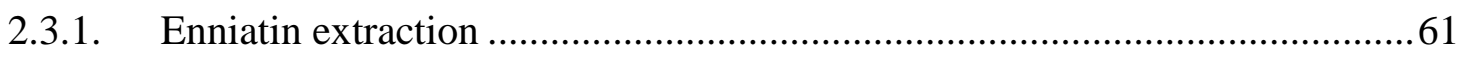

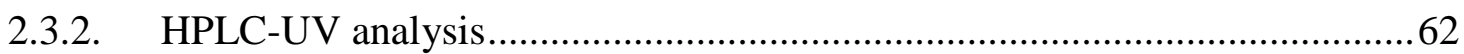

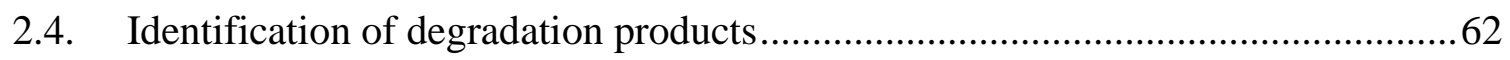

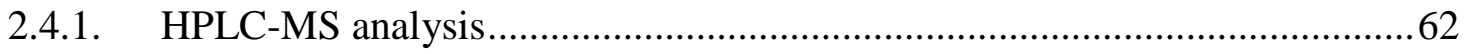

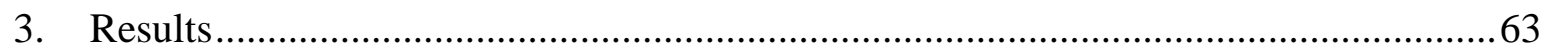


3.1. Selection of Microorganisms from mixed cultures-Binocular microscopic observation of the microtiter plates

3.2. Microorganisms isolated from mixed cultures .................................................65

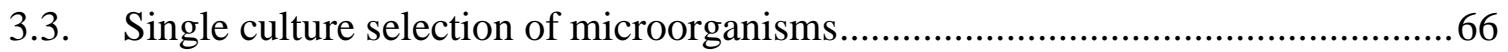

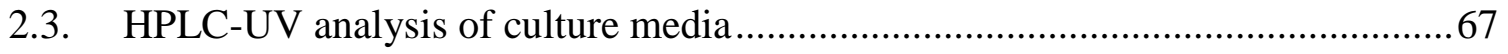

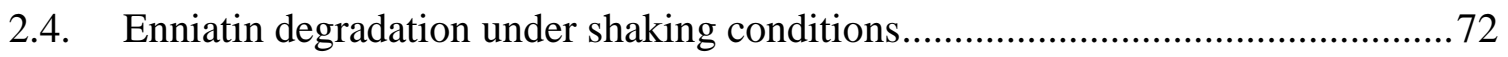

2.5. Identification of degradation products using HPLC-MS .................................... 73

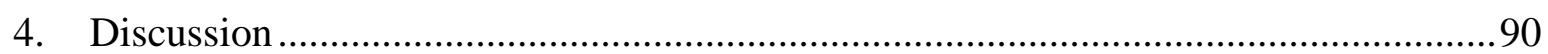

4.1. Selection of microorganisms from mixed cultures .............................................90

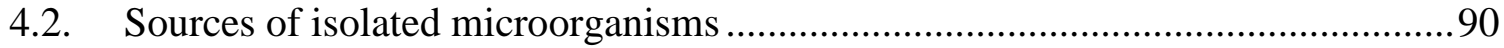

4.3. HPLC-UV chromatograms and enniatin reduction in culture media ....................91

4.4. Identification of degradation products using HPLC-MS .................................... 92

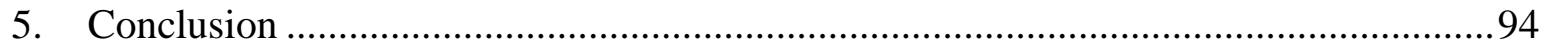

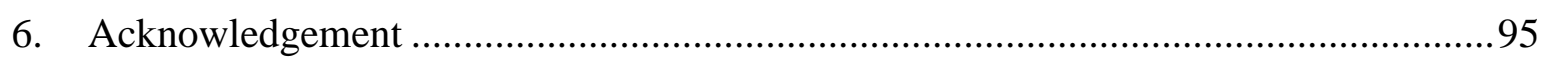

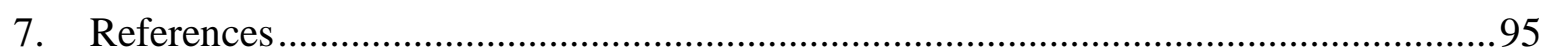

\section{Chapter 4}

Identification of a bacterium and two fungi capable of degrading enniatins..................101

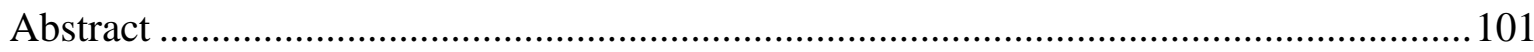

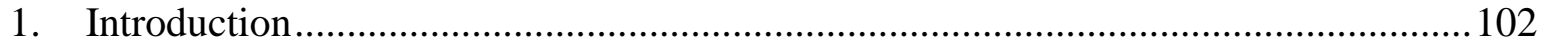

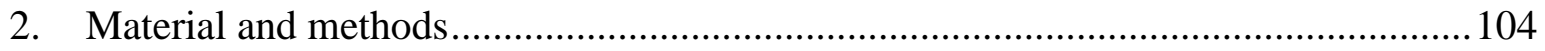

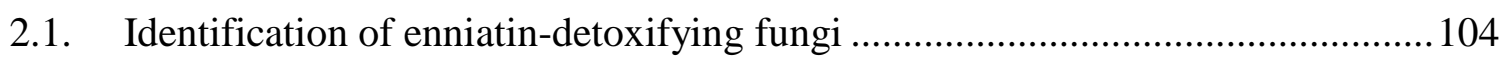

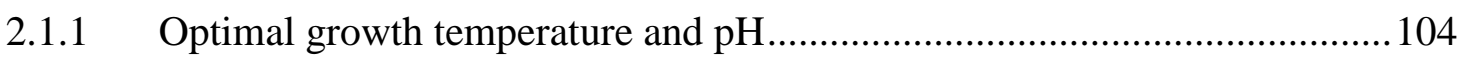

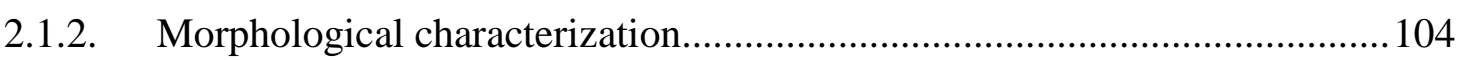

2.1.3. Molecular characterization of fungi isolates ............................................ 105

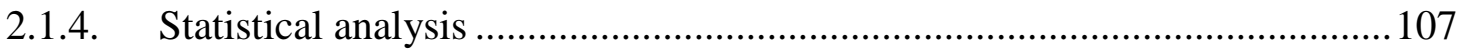

2.2. Identification of enniatin-detoxifying bacterium............................................. 107

2.2.1. Morphological characterization and optimal growing temperature of the

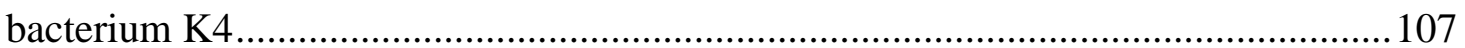

2.2.2. Biochemical characterization of the bacterium K4 f................................. 108

2.2.3. Molecular identification of bacterial isolate.............................................. 109

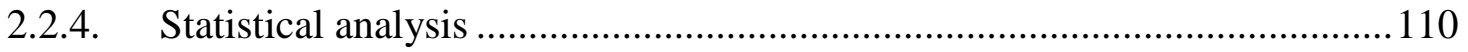

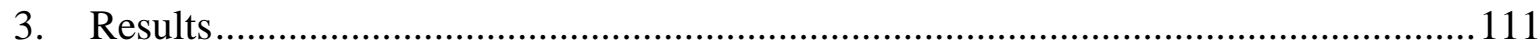

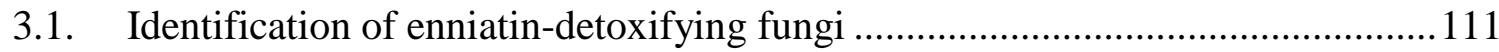

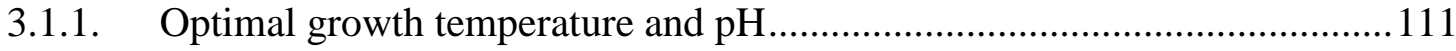

3.1.2. Morphological characterization of fungal isolates .................................... 113

3.1.3. Molecular characterization of fungal isolates ......................................... 117 
3.2. Identification of enniatin detoxifying bacterium 120

3.2.1. Morphological characterization and optimal growing temperature of the bacterium $\mathrm{K} 4$ 120

3.2.2. Biochemical characterization of the bacterium $\mathrm{K} 4$................................... 121

3.2.3. Molecular identification of bacterial isolate................................................ 122

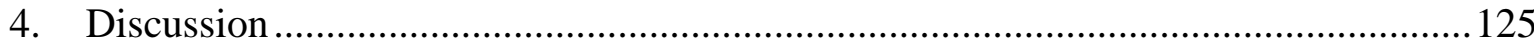

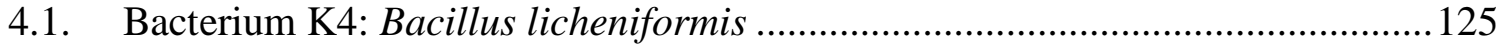

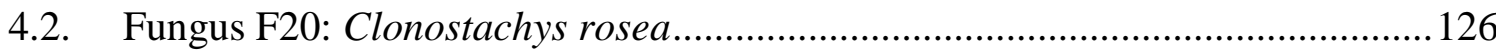

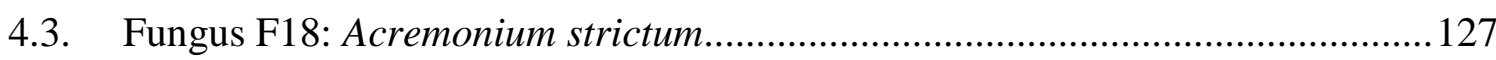

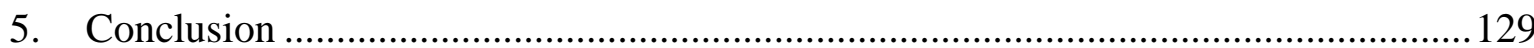

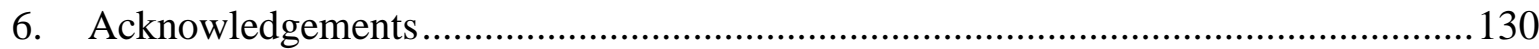

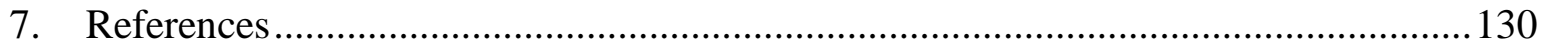

\section{Chapter 5}

General discussion ..................................................................................................................... 137

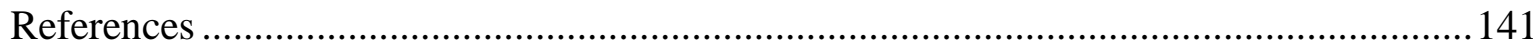

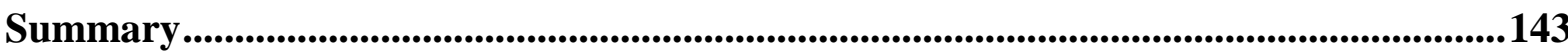

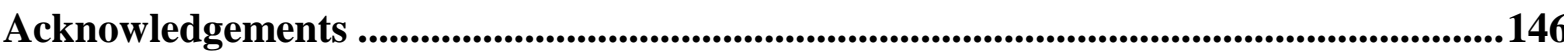

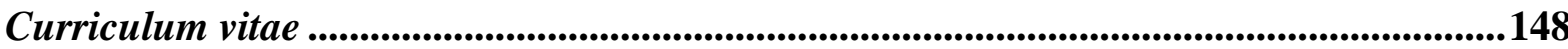

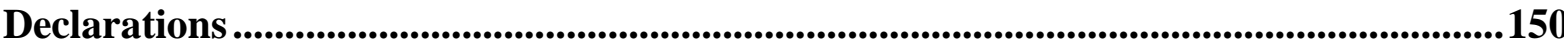




\section{Chapter 1}

\section{General background}

\section{Introduction}

Enniatins are secondary metabolites produced by several Fusarium species such as $F$. orthocerar (Gäumann et al., 1947) F. tricinctum, F. lateritium, F. oxysporum, F. avenaceum (Blais et al., 1992; Herrmann et al., 1996; Wang et al., 2013) and a few other genera of fungi such as Alternaria (Mckee et al., 1997), Verticillium (Nilanonta et al., 2003) and Halosarpheia (Lin et al., 2002). More recently, Zobel et al. (2005) also demonstrated the production of enniatin B by a recombinant Bacillus subtillis and Richter et al. (2014) who engineered Aspergillus niger for the production of several variants of enniatin. According to Jestoi et al. (2008) the main enniatin producers are $F$. subglutinans, $F$. proliferatum and $F$ tricinctum.

Enniatins are a group of cyclic hexadepsipepdides which have alternated residues of three Nmethyl amino acids in their structure, often valine, leucine and isoleucine and three hydroxyisovaleric acids. The branched chain amino acids are linked by peptide and ester bonds. Sy-Cordero et al. (2012) reported that 29 variants of enniatins have been isolated and characterized, they differ from each other in their amino acid and hydroxyacid composition, but more variants could be found due to the multiple possibilities of incorporation of amino acids and the hydroxyl acids by the enzyme enniatin synthetase and D-2-hydroxyisovalerate dehydrogenase. Beauvericin and destruxin mycotoxins are structurally similar to enniatins. Destruxins contain only one hydroxy acid and five amino acid residues in their structure (Pedras et al., 2002), while beauvericin is composed of alternating $\mathrm{N}$-methylphenylalanine and hydroxy acid residues (Hamill et al. 1969).

High concentrations of enniatins in food commodities have been reported in several countries mainly in northern Europe and the Mediterranean. The main tested foods were cereal and cereal-based products. Enniatins B, B1, B4, A and A1 were quantified in baby food including infant formula milks, dairy products (cheese and yogurt), cereal based baby food, fruits and vegetables compotes, fruits and vegetable puree from the Italian market. The detected concentrations ranged from 11.8 to $832 \mu \mathrm{g} / \mathrm{kg}$ in cereal-based baby food, containing wheat as 
main cereal. The highest value was enniatin B (Juan et al., 2014). According to a study carried out by Serrano et al. (2013), organic pasta from Spain was more contaminated with enniatin (A, A1, B1 and B) than the conventional counterpart. Although the majority of the enniatin concentrations found in organic and conventional dry or fresh samples in Spain were below $25 \mu \mathrm{g} / \mathrm{kg}$, concentrations up to $979.56 \mu \mathrm{g} / \mathrm{kg}$ of enniatin A, A1 B1 and B were detected in conventional dry pasta. In cereal (wheat, barley, maize, sorghum) and cereal- derived products (mainly pasta and couscous) originating from Tunisia, high levels of enniatins A, A1, B and B1 were found. A maximum concentration of the four enniatin variants of 683.9 $\mathrm{mg} / \mathrm{kg}$ was detected in sorghum. Among the tested enniatin variants, enniatin A1 had the highest prevalence with concentrations ranging from 11.1 to $480 \mathrm{mg} / \mathrm{kg}$ (Oueslati et al., 2011). A survey carried out by Uhlig et al. (2006) in Norwegian oat, barley and wheat grain samples from 2000 to 2002 revealed that concentrations of enniatin B above $1000 \mu \mathrm{g} / \mathrm{kg}$ are common and similarly, enniatin B occurred with the highest prevalence and a maximum concentration of $5800 \mu \mathrm{g} / \mathrm{kg}$ was found in wheat. Finnish barley and wheat were also investigated by Yli-Mattila et al. (2004, 2008, 2009), Jestoi et al. (2004) and Hietaniemi et al. (2016). Besides the studies made on the occurrence of enniatins in cereal and cereal-based products, enniatins have been also been found in egg and wine from Finland and China respectively. Enniatin B and B1 were found in whole eggs and yolk egg in trace-levels (< limit of quantification) of samples from 2004 (Jestoi et al., 2009). Enniatin B (Wang et al., 2011) was detected in wine samples made out of grapes harvested in a vineyard contaminated by Fusarium tricinctum (Corda). Nuts and dried fruits from Spain were screened for the occurrence of enniatins by Tolosa et al. (2013), the samples included peanuts fruit and shell, almonds fruit and shell, Pistachios fruits and shell, walnuts fruit and shell, fried maize, hazelnuts fruit and shell, sunflower seeds fruit and shell, dried fruits (dried apricots, dried figs, dried raisins, dried plums, blueberries and dates). Enniatin A and B were the most prevalent in the analyzed samples. The percentages of total enniatin contamination of samples analyzed ranged were $35.7 \%, 50 \%$ and $83.3 \%$ for dried fruits, nuts and dates respectively. The highest concentration of enniatins (A, A1, B and B1) was found in peanuts, almonds and dates reaching a maximum of $1.855 \mathrm{mg} / \mathrm{kg}, 1.323 \mathrm{mg} / \mathrm{kg}$ and $1.494 \mathrm{mg} / \mathrm{kg}$ respectively.

Various biological activities of enniatins have been described. Some damaging effects on plants have been associated to enniatins, for instance they affect water uptake by cells in tomato shoots, leading to toxic wilt and necrosis (Gäumann et al., 1960). An in vitro toxicity 
of a mixture of enniatins $\mathrm{A}, \mathrm{A} 1, \mathrm{~B}$ and $\mathrm{B} 1$ characterized by necrosis was also observed in potato tubers, depending on the incubation time and the amount of enniatins applied (Herrmann et al., 1996). Moreover, enniatins (mainly B and B1) concentrations ranging from $10-80 \mu \mathrm{g} / \mathrm{mL}$ have been directly associated to a growth reduction of the wheat seed during germination, characterized by a higher inhibition of the root elongation compared to the leaf development (Burmeister and Plattner, 1987). Logrieco et al. (1998) associated some insecticidal activities and apoptosis in mammalian cells to beauvericin exposure in mammals. The toxicity of enniatins is assumed to derive from their ionophoric properties and to their action as uncouplers of oxidative phosphorylation (Shemyakin et al., 1969). Individual and combined toxicity of enniatins A, A1, B and B1 were evaluated with CHO-K1. The highest half maximal inhibition concentration $\left(\mathrm{IC}_{50}\right)$ was attributable to enniatin $\mathrm{B}$. Furthermore, the mixture of enniatin $\mathrm{A}+\mathrm{B} 1, \mathrm{~A} 1+\mathrm{B}, \mathrm{B}+\mathrm{B} 1$ led to an increase of the observed effect ( $\mathrm{Lu}$ et al., 2013)

Besides the above mentioned harmful effects of enniatins, they also possess some antibiotic properties which have been exploited in the production of an antibiotic known as fusafungine -a mixture of enniatin A, B and C-produced by Fusarium lateritium (Moffat, 1986). More recently, some researchers confirmed the role of enniatins as antibiotic. Enniatins B, B1 and $\mathrm{G}$ were effective against some bacterial strains (Bacillus subtilis, Candida albicans, Trychosporom cutaneum and Cryptococcus neoformans) in agar diffusion test by lowering the activity of the 12- Lipoxygenase and therefore proved an antibiotic activity (Firákova et al., 2008). Another study highlights the fact that enniatins $\mathrm{J}_{1}$ and $\mathrm{J}_{3}$ could be potentially effective antibacterial agents against several pathogenic and lactic acid bacteria (Sebastià et $a l ., 2011)$. Enniatin B induced the inhibition of the microorganisms of some normal intestinal tract pathogens such as Escherichia coli, Salmonella enterica, Clostridium perfringens, Pseudomonas aeruginosa (Meca et al., 2011). The antibiotic properties conferred by fusafungine make it the active compound of a medicine commercially available in Germany under the name Locabiosol. Locabiosol which is used for the treatment of bacterial upper respiratory infections for example for a sore throat or a blocked nose (German-Fattal and Mösges, 2004).

The broad range of biological activity of enniatins is ascribed to their ionophoric properties. It was demonstrated that a mixture of enniatin A, A1, B and B1 is easily incorporated into the 
cell membrane through passive channels and form cation selective pores. Enniatins induce changes in intracellular ion concentration and disrupt cell function (Kamyar et al., 2014).

\section{In vitro production of enniatins}

Early in 1974, Audhya and Russell produced enniatins using Fusarium sambucinum in chemically defined liquid media. The enniatin production was favored by various factors, such as supplementation with lactose and tryptone, moderate temperatures and light exposure. Quantitative paper chromatography or spectrophotometry was used to quantify enniatin A with a yield of $1.6 \mathrm{~g} / \mathrm{L}$. Madry et al. (1983) carried out a similar study where the impact of various media components were studied for the production of enniatins using $F$. oxysporum in liquid culture. The production of enniatins was promoted by the addition of sucrose, glucose and amino acids (valine, leucine and isoleucine) leading to a yield up to $5 \mathrm{~g} / \mathrm{L}$ of several enniatins after analysis of the chloroform extract using spectrophotometry methods. The first study about the production of enniatin by $F$. tricinctum was reported by Burmeister and Plattner (1987). F. tricinctum was incubated on a semi solid medium of corn grit for 16 days at ambient temperature and cultures were extracted using methanol and analyzed using mass spectrometry/ mass spectrometry (MS/MS) to give a yield of 3-3.270 $\mu \mathrm{g} / \mathrm{g}$ of enniatin B and B1.

\subsection{Fermentation substrate}

Initially the main substrates used for enniatin productions were chemically defined liquid media (Audhya and Russell, 1974; Madry et al., 1983; Strongman et al., 1988; Visconti et al., 1992; Herrmann et al. 1996), and semi solid media such as corn grit (Burmeister and Plattner 1987). More recently, several authors have reported the production of high amounts of enniatins using solid matrices such as rice (Moretti et al. 2007), corn (Meca et al. 2010), or several solid substrates including white beans, soybeans, radish, potato, wheat, corn and rice (Wang et al. 2013). According to Wang et al. (2013) enniatins production was highest in the white bean media without any supplementation of amino acids $(1.365 \mathrm{mg} / \mathrm{L}$ enniatins $\mathrm{A}, \mathrm{A} 1$, B1 and B after 18 days). 


\subsection{Extraction, detection and quantification}

Enniatins are strong hydrophobic compounds and their extraction from fungal extracts have been done using mild to strong hydrophobic solvents such as water/methanol and ethyl acetate (Cuomo et al., 2013), methanol (Burmeister and Plattner 1987), acetonitrile (Serrano et al., 2013), hexane (Firáková et al., 2008), chloroform media (Audhya and Russell, 1974; Madry et al., 1983), ethyl acetate (Wang et al., 2013). Among the techniques used so far for the detection and quantification of enniatins from extracts are quantitative paper chromatography or spectrophotometry (Audhya and Russell, 1974; Madry et al., 1983), mass spectrometry/mass spectrometry (Burmeister and Plattner 1987), HPLC-UV (Firáková et al., 2008 Wang et al., 2013), LC-MS-MS (Sørensen et al. 2008), LC-MS-LIT (Serrano et al., 2013).

\subsection{Purification}

Generally preparative chromatographic techniques have been used for the purification of enniatins from fungal extract. Visconti et al. (1992) reported the purification of enniatins A, A1, B, B1, B2, B3 and B4 from liquid culture of Fusarium acuminatum strains, these authors made a liquid-liquid extraction of the culture broth using hexane, then separated enniatins using two-step preparative reverse phase HPLC and acetonitrile water as mobile phase to get pure enniatins in the range of 2 to $30 \mathrm{mg}$ from $2 \mathrm{~L}$ liquid culture. Enniatins A, A1, B and B1 were purified from Fusarium tricinctum grown on solid corn medium using low pressure liquid chromatography, followed by semipreparative liquid chromatography both on reverse stationary phase to obtain enniatins with a yield varying from 20 to $56 \mathrm{mg}$ from $100 \mathrm{~g}$ solid corn medium (Meca et al., 2010). Similarly, The purification of enniatins A, A1, B, B1, B4 and $\mathrm{J}$ produced by Fusarium tricinctum in solid corn culture has been reported by Cuomo et al. (2013), the fermented solid culture was extracted first using water/methanol (50/50, v/v), then by ethyl acetate and the extract was separated using a two steps process including low pressure liquid chromatography (normal phase silica gel and an isocratic gradient of chloroform/isopropylalcohol 80/20 v/v) and semi-preparative HPLC (a Gemini phenomenex column and a gradient elution with water and acetonitrile) to get mixtures of six enniatins with yields of 30-300 mg per compound. 


\section{Mycotoxin decontamination strategies}

In order to limit the exposure of human and animals to mycotoxins in food and feed, methods for mitigating the mycotoxin content have been developed. The techniques used for detoxification of mycotoxins in food can be classified into three categories: physical, chemical and biological methods (He and Zhou, 2010).

\subsection{Physical detoxification}

Among the physical methods used to remove mycotoxins a method based on the separation of zearalenone-deoxynivalenol- infected wheat, corn and sorghum grains was studied by Huff $e t$ al., (1982) and Babadoost et al., (1987). Milling provides a partial removal of mycotoxins as a higher amount will be found in the bran than in the flour, but the process does not destroy mycotoxins. It was shown that during dry milling of corn, fumonisin B1 was found in highest amounts in the bran fraction that is used as animal feed followed by the germ fraction, which may be used as animal feed or for oil extraction (Katta et al., 1997). Aflatoxin B1 and Ochratoxin A were relatively stable at boiling temperatures of the mash cooking step, but were more sensitive to mash malting (protein hydrolysis) with removal of 12-27\% (Chu et $a l ., 1975$ ). A decrease of the enniatin (A, A1, B and B1) content of $30 \%$ was observed during bread baking, while no enniatins were found in final beers probably due to their high hydrophobic character (Vaclavikova et al., 2013). In a study of the variation of the enniatin content in naturally contaminated wheat and rye grains during milling, the distribution of enniatins B and B1 was about 70- 82\% in the bran, while the rest was present in the flour. Similarly, 24 hour sourdough fermentation at $40^{\circ} \mathrm{C}$ led to $17-19 \%$ and $10 \%$ reduction of enniatins and beauvericin respectively compared to $7-10 \%$ enniatins reduction at $30^{\circ} \mathrm{C}$. Generally, enniatins and beauvericin concentrations were $25-41 \%$ lower in the bread compared to unprocessed material (Hu et al., 2014). Food processing techniques can reduce the amount of some mycotoxins in food, by diluting and distributing mycotoxins into certain fractions, but this might be used for animal feed and then still enter the human food chain. The process of reducing mycotoxins in food using heat treatments is temperature and time dependant. Most mycotoxins are moderately stable in most food processing systems (Bullerman and Bianchini, 2007). 


\subsection{Chemical detoxification}

Chemical detoxification involving alkalis and acids to deactivate aflatoxins was developed in raw material intended for animal and human food products including cottonseed, peanuts, coconuts and corn grains. Treatment of one fraction of the raw material was made with a base and the other fraction was treated with an acid, then the two fractions are combined to bring the $\mathrm{pH}$ back to neutral (Emetco, 1995).

\subsection{Biological detoxification}

Biological detoxification can be defined as the enzymatic degradation or transformation of toxins that leads to less toxic products (Bhatnagar, 1991). Methods of detoxification of mycotoxins using microorganisms generally used enrichment cultures based on a single substrate. Fumonisins B1 and B2 were detoxified as unique carbon source by fungi Exophilia spinifera (ATCC 74269) and Rhinocladiella atrovirens (ATCC 74270) and the bacterium ATCC 55552, the microorganisms were isolated from field-grown maize kernels and stalks (Duvick and Rood, 1998). In the scientific literature a US patent on the biological detoxification of beauvericin is available. A method allowing the reduction of beauvericin in vitro and in vivo was found and beauvericin contaminated corn was detoxified employing strains of Norocardia glubera (Duvick and Rood, 2000). The detoxifying action of fungus $C$. rosea was demonstrated to be due to the enzyme zearalenone lactonohydrolase that cleaves the lactone rings from the backbone and transforms zearalenone to a far less potent compound (Kakeya et al., 2002). Two Pseudomonas strains were able to degrade zearalenone by about $57-68 \%$ using an enrichment technique with zearalenone as the sole carbon source (Tan et al., 2014). A bacterium isolated from a soil sample from a wheat field classified as Nocardioides was able to completely remove $1000 \mu \mathrm{g} / \mathrm{mL}$ of DON after 10 incubation days from a liquid culture medium (Ikunaga et al., 2011). The use of Saccharomyces strains have led to a reduction of enniatins A, A1, B and B1 in vitro or in food system, LC-MS-LIT was used to find three degradation products (Roig et al., 2013). A reduction of enniatins A, A1, B and B1 was induced by six Bacillus subtilis strains in tryptic soy broth after incubation for 48 hours at $37^{\circ} \mathrm{C}$ under aerobic conditions. Two degradation products were identified using LC-MS resulting from the loss of hydroxyisovaleric acid in the structures of enniatin B and B1 (Meca et al., 2014). 
Food processing techniques such as sorting, trimming, cleaning, milling, brewing, cooking, baking, frying, roasting, canning, flaking, alkaline cooking, nixtamalization and extrusion may have an impact of the mycotoxin content in food (Bullerman and Bianchini, 2007). But these methods present some disadvantages like possible losses of important nutrients. From the opinion of many scientists, biological detoxification is a promising technique enabling the removal of mycotoxins under mild conditions and without the use of harmful chemicals and significant losses in nutritive value and palatability of the decontaminated feed and food (Karlovsky, 1999; Bata and Lásztity, 1999; Kabak et al., 2006; Wu et al., 2009). Furthermore, the genes responsible for some of the detoxification activities can be cloned and expressed in heterologous hosts.

\section{The complex of problems and objectives}

Due to the relatively high price of enniatins as raw products on the market (and in our case the large quantity involved in the detoxification assay), it has been common for many authors to purify the enniatins that were required for further use such as for toxicity tests for instance. Moreover, some authors have attempted to optimize the in vitro conditions for the production of enniatins.

The first objective of this study will be to optimize the enniatin production by examining two strains of $F$. tricinctum and to find the best harvesting time point after incubation of the strains on solid white bean media. Then enniatins will be purified from the fungal crude extract.

The data reported in the literature about enniatins contents in food products are obtained using different extraction techniques as well as analytical instruments and thus might vary from each other in terms on sensitivity and precision. However, the values in general are very high and should raise concern about the long term effect of those metabolites on consumer health, data about the chronic exposure of humans through diet to such high concentrations of enniatins are still lacking. Maximum levels of Enniatins concentrations in food has not been set by the European Union suggesting the ignorance related to the possible risks to which the population may be exposed. This has led to some investigations targeting the degradation of enniatin in food by some microorganisms. 
In the second part of this thesis, microorganisms (bacteria and/or yeast and/or fungi) able to degrade enniatins into less toxic compounds will be screened, the degradation products will be identified and the degradation reaction will be deduced.

Since random screening of unknown microorganisms will be used as a strategy to isolate the microorganisms with the ability to degrade enniatins into less toxic compounds, the third part of this work will consist in the taxonomical identification of the active microorganisms with the aid of phenotypic and molecular means. 


\section{References}

Audhya, T.K. and Russell, D.W. (1974): Production of enniatins by Fusarium sambucinum: selection of high-yield conditions from liquid surface cultures, Journal of General Microbiology, 82: 181-190.

Babadoost, T.M., Hagler Jr, W.M., Bowman, D.T., Nelson, P.E. (1987): Field contamination of sorghum with zearalenone and deoxynivalenol in North Carolina, Density segregation to remove mycotoxins, Biodetetioration Research, 1: 99-1095.

Bata, Á. and Lásztity, R. (1999): Detoxification of mycotoxin-contaminated food and feed by microorganisms, Trends in Food Science and Technology, 10: 223-228.

Bhatnagar, D., Lillehoj E.B., Bennett J.W. (1991): Biological detoxification of mycotoxins In: Smith, JE, Henderson, RS, eds. Mycotoxins and Animal Foods, Boston: CRC Press, 816826.

Blais, L.A., Apsimon, J.W., Blackwell, B.A., Greenhalgh, R., Miller, J.D. (1992): Isolation and characterization of enniatins from Fusarium avenaceum Daom- 196490, Canadian Journal of Chemistry, 70: 1281-1287.

Bullerman, L.B., Bianchini, A., (2007): Stability of mycotoxins during food processing, International Journal of Food Microbiology, 119: 140-146.

Burmeister, H.R., Plattner, R.D. (1987): Enniatin production by Fusarium tricinctum and its effect on germinating wheat seeds, Phytopathology, 77: 1483-1487.

Chu, F.S., Chang, C.C., Ashoor, S.H., Prentice, N. (1975): Stability of aflatoxin B1 and ochratoxin A in brewing, Applied Microbiology, 29(3): 313-316.

Cuomo, V., Randazzo, A., Meca, G., Moretti, A., Cascone, A., Eriksson, O., Novellino, E., Ritieni, A. (2013): Production of enniatin A, A1, B, B1, B4, J1 by Fusarium tricinctum in solid corn culture: structural analysis and effect on mitochondrial respiration, Food chemistry, 140: 784-793.

Duvick, J., Rood, T. A. (2000): Beauvericin detoxification methods using bacteria, US 6126934 A. 
Duvick, J., Rood, T., Wang, X. (1998): Fumonisin detoxification enzymes, US 5716820 A.

Emetco, B.V. (1995): Method for de-activating mycotoxin. EPO4507221B1.

Firáková, S, Šturdíková, M., Liptaj, T., Prónayová, N., Bezáková, L., B. Proksa, B. (2008): Enniatins produced by Fusarium dimerum, an endophytic fungal strain, Pharmazie, 63: 539541.

Gäumann, E., Naef-Roth, S., Kern, H. (1960): Zur phytotoxischen Wirksamkeit der Enniatine, Phytopathologie Zeitung, 40: 45-51.

German-Fattal, M., Mösges, R. (2004): How to improve current therapeutic standards in upper respiratory infections: value of fusafungine, Current Medical Research and Opinion, 20(11): 1769-1776.

Hamill R.L., Higgens, C.E., Boaz H.E, Gorman M., (1969): Structures of beauvericin, a new depsipeptide antibiotic toxic to alternaria salina, Tetrahedron Letters, 10: 4255- 4258.

He, J. and Zhou, T. (2010): Patented techniques for detoxification of mycotoxins in feeds and food matrices, Recent Patents on Food, Nutrition and Agriculture, 2: 96-104.

Herrmann, M., Zocher, R., Haese, A. (1996): Enniatin production by Fusarium strains and its effect on potato tuber tissue, Applied and Environmental Microbiology, 62 (2): 393-398.

Hietaniemi, V, Rämö, S., Yli-Mattila, T., Jestoi, M., Peltonen, S., Kartio, M., Sieviläinen, E., Koivisto, T. and Parikka, P.: (2016): Updated survey of Fusarium species and toxins in Finnish cereal grains, Food Additives and Contaminants: Part A, 33(5): 831-848.

Hu, L., Koehler P., Rychlik M. (2014): Effects of sourdough processing and baking on the content of enniatins and beauvericin in wheat and rye bread, European Food Research and Technology, 238: 581-587.

Huff, W.E. and Hagler Jr, W.M. (1982): Evaluation of density segregation as a means to estimate the degree of Aflatoxin contamination of corn, Cereal Chemistry, 59: 152-153.

Ikunaga, Y., Sato, I., Grond, S., NUmaziri, N., Yoshida, S., Yamaya, H. et al. (2011): Nocardioides sp. strain WSN05-2, isolated from a wheat field, degrades deoxynivalenol, producing the novel intermediate 3-epi- deoxynivalenol, Applied Microbiology, 89: 419-427. 
Jestoi, M., Rokka, M., Yli-Mattila, T., Parikka, P., Rizzo A., Peltonen, K. (2004): Presence and concentrations of the Fusarium-related mycotoxins beauvericin, enniatins and moniliformin in Finnish grain samples, Food Additives and Contaminants, 21(8): 794-802.

Jestoi, M, Rokka, M, Järvenpää, E, Peltonen, K. (2009): Determination of Fusarium mycotoxins beauvericin and enniatins (A, A1, B, B1) in eggs of laying hens using liquid chromatography-tandem mass spectrometry (LC-MS/MS), Food Chemistry, 115: 1120-1127.

Jestoi, M. (2008): Emerging Fusarium-mycotoxins fusaproliferin, beauvericin, enniatins and moniliformin- a review, Critical reviews in Food Science and Nutrition, 48: 21-49.

Juan, C., Assunta, R., Mañes J., Ritieni, A. (2014): Presence of mycotoxins in commercial infant formulas and baby foods from Italian market, Short communication, Food Control, 39: 227-236.

Kabak, B., Dobson, A.D., Var, I. (2006): Strategies to prevent mycotoxin contamination of food and animal feed: a review, Critical Review in Food Science and Nutrition, 46(8): 593619.

Kakeya, H., Takahashi-Ando, N., Kimura, M., Onose, R., Yamaguchi, I., Osada, H. (2002): Biotransformation of the mycotoxin zearalenone, to a non-oestrogenic compound by the fungal strain of Clonostachys sp. Bioscience, Biotechnology and Biochemistry, 66:27232726.

Kamyar, M., Rawnduzi, P., Studenik, C.R., Kouri, K., Lemmens- Gruber, R. (2004): Investigation of the electrophysiological properties of enniatins, Archives of Biochemistry and Biophysics, 428: 215- 223.

Karlovsky, P. (1999): Biological Detoxification of Fungal Toxins and its Use in Plant Breeding, Feed and Food Production, Natural Toxins, 7: 1-23.

Katta, S.K., Cagampang, A.E., Jackson, L.S., Bullerman, L.B. (1997): Distribution of Fusarium molds and fumonisins in dry-milled corn fractions, Cereal Chemistry, 74: 858-863.

Lin, Y., Wang, J., Wu, X., Zhou, S., Vrijmoed, L.L.P., Jones, E.B.G. (2002): A novel compound enniatin $\mathrm{G}$ from the mangrove fungus Halosarpheia sp. (strain \#732) from the south china sea, Australian Journal of Chemistry, 55: 225-227. 
Madry, N., Zocher, R., Kleinkauf, H. (1983): Enniatin production by Fusarium oxysporum in chemically defined media, European Journal Applied Microbiology and Biotechnology, 17: 75-79.

Mckee, T.C., Bokesch, H.R., McCormick, J.L., Rashid, M.A., Spielvogel, D. et al. (1997): Isolation and characterization of new anti-HIV and cytotoxic leads from plants, marine and microbial origins, Journal of Natural Products, 60(5): 431-438.

Meca, G., Roig, M., Ferrer, E., Mañes, J. (2014): Degradation of the bioactive compounds enniatins A, A1, B, B1 employing different strains of Bacillus subtilis, Journal of Food Processing and Technology, 5: 334.

Meca, G., Ruiz, M.J., Soriano, J.M., Ritieni, A., Moretti, A., Font, G., Mañes, J. (2010): Isolation and purification of enniatins $\mathrm{A}, \mathrm{A} 1, \mathrm{~B}, \mathrm{~B} 1$, produced by Fusarium tricinctum in solid culture and cytotoxicity effects on Caco-2 cells, Toxicon, 56: 418-424.

Meca, G., Sospedra, I., Valero, M.A., Mañes, J., Front, G., Ruiz, M.J. (2011): Antibacterial activity of the enniatin B, produced by Fusarium tricinctum in liquid culture, and cytotoxic effects on Caco-2 cells, Toxicology Mechanisms and Methods, 21(7): 503-512.

Moffat, A.C. (1986): Clarke's isolation and Identification of Drugs, The pharmaceutical Press, London.

Moretti, A., Mulè, G., Ritieni, A., Logrieco, A. (2007): Further data on the production of beauvericin, enniatins and fusaproliferin and toxicity to Artemia salina by Fusarium species of Gibberella fujikuroi species complex, International Journal of Food Microbiology, 118:158-163.

Nilanonta, C., Isaka, M., Chanphen, R., Thong-orn, N., Tanticharoen, M., Thebtaranonth, Y. (2003): Unusual enniatins produced by insect pathogenic fungus Verticillium hemipterigenum: isolation and studies on precursor-directed biosynthesis, Tetrahedron, 59: 1015-1020.

Oueslati, S., Meca, G., Mliki, A., Ghorbel A., Jordi Mañes, J. (2011): Determination of Fusarium mycotoxins enniatins, beauvericin and fusaproliferin in cereals and derived products from Tunisia, Food Control, 22: 1373-1377. 
Pedras, M.S., Irina Zaharia, L, Ward, D.E. (2002): The dextruxins: synthesis, biosynthesis, biotransformation and biological activity, Phytochemistry, 59: 579- 596.

Richter, L., Wanka, F., Boecker, S., Storm, D., Kurt, T. et al. (2014): Engineering of Aspergillus niger for the production of secondary metabolites, Fungal Biology and Biotechnology, 1:4.

Roig, M., Meca G., Ferrer, E., Mañes J. (2013): Reduction of the enniatins A, A1, B, B1 by an in vitro degradation employing different strains of probiotic bacteria: Identification of degradation products by LC- MS- LIT, Toxicon, 70: 44-53.

Sebastià, A., Meca, G., Soriano, J.M., Mañes, J. (2011): Antibacterial effects of enniatins J 1 and $\mathrm{J}_{3}$ on pathogenic and lactic acid bacteria, Brief Communication, Food and Chemical Toxicology, 49: 2710-2717.

Serrano, A.B., Meca, G., Font, G., Ferrer, E. (2013): Degradation study of enniatins by liquid chromatography-triple quadrupole linear ion trap mass spectrometry, Food Chemistry, 141: 4215-4225.

Shemyakin, M.M., Ovchinnikov, Y.A., Ivanov, N.T., Antonov, V.K., Vinogradova, E.I., Shkrob, A.M., Malenkov, G.G., Evstratov, A.V., Laine, I.A., Melnik, E.I., Ryabova, I.D. (1969): Cyclodepsipeptide as chemical tools for studying ionic transport through membranes, Journal of Membrane Biology, 1: 402- 430.

Sørensen, J.L., Nielsen, K.F., Rasmussen, P.H., Thrane, U. (2008): Development of a LCMS/MS method for the analysis of enniatins and beauvericin in whole fresh and in ensiled maize samples, Journal of Agricultural and Food Chemistry, 56(21): 10439-10443.

Strongman, D. B., Strunz, G. M., Giguere, P., Yu. C.-M., Calhoun, L. (1988): Enniatins from Fusarium avenaceum isolated from balsam fir foliage and their toxicity to spruce budworm larvae, Choristoneura fumiferana (Clem.), Journal of Chemical Ecology, 14(3): 753-764.

Sy-Cordero, A.A., Pearce, C.J., Oberlies, N.H. (2012): Revisiting the enniatins: a review of their isolation, biosynthesis, structure determination and biological activities, The Journal of Antibiotics, 65(11): 541-549. 
Tan, H., Hu, Y., He, J., Wu, L., Liao, F., Luo, B., et al. (2014): Zearalenone degradation by two Pseudomonas strains from soil, Mycotoxin Research, 30(4): 191-196.

Tolosa, J, Font, G. Mañes, J, Ferrer, E. (2013): Nuts and dried fruits: Natural occurrence of emerging Fusarium mycotoxins, Food control, 33: 215-220.

Uhlig, S., Torp, M., Heier, B. (2006): Beauvericin and enniatin A, A1, B and B1 in Norwegian grain: a survey, Food chemistry, 94: 193-201.

Vaclavikova, M., Malachova, A., Veprikova, Z., Dzuman, Z., Zachariasova, M. (2013): "Emerging" mycotoxins in cereals processing chains: Changes of enniatins during beer and bread making, Food chemistry, 136: 750-757.

Visconti, A., Blais, L.A., Apsimon, J.W., Greenhalgh, R., Miller, J.D. (1992): Production of enniatins by Fusarium acuminatum and Fusarium compactum in liquid culture: isolation and characterization of three new enniatins B2, B3, and B4, Journal of Agricultural and Food Chemistry, 40: 1076-1082.

Wang, J.P., Debbab, A., Hemphill, C.F., Proksch, P. (2013): Optimization of enniatin production by solid-phase fermentation of Fusarium tricinctum, Zeitschrift für Naturforschung C, 68(5-6): 223-230.

Wang, Z., Lin, X., Yu, M., Huang, Y., Qian, X., Shen, Y. (2011): Wine contamination by mycotoxin enniatin B from Fusarium tricinctum (Corda) Sacc., Journal of Food, Agriculture and Environment, 9: 182-185.

Wu, Q.H., Jezkova, A., Yuan, Z.H., Pavlikova, L., Dohnal, V., Kuca, K. (2009): Biological degradation of aflatoxins, Drug Metabolism Reviews, 41(1): 1-7.

Yli-Mattila, T., Paavanen-Huhtala, S., Jestoi, M., Parikka, P., Hietaniemi, V., Gagkaeva, T., Sarlin,T., Haikara, A., Laaksonen, S. and Rizzo, A. (2008): Real-time PCR detection and quantification of Fusarium poae, F.graminearum, F. sporotrichioides and F. langsethiae in cereal grains in Finland and Russia, Archives of Phytopathology and Plant Protection, 41(4): 243-260.

Yli-Mattila, T., Paavanen-Huhtala, S., Parikka, P., Hietaniemi, V., Jestoi, M., Rizzo, A., (2004): Real-time PCR detection and quantification of Fusarium poae as compared to 
mycotoxin production in grains in Finland, In: Canty S.M., Boeing T., Versdahl K., Wardwell J., Ward R.W (editors), Proceedings of the 2nd International Symposium on Fusarium Head Blight, Orlando, 422-425.

Yli-Mattila, T., Parikka, P., Lahtinen, T., Ramo, S., Kokkonen, M., Rizzo, A., Jestoi, M., Hietaniemi, V. (2009): Fusarium DNA levels in Finnish cereal grains, In: Gherbawy Y., Mach R.L., Rai M.K., editors, Current Advances in Molecular Mycology, Nova Science Publishers, Inc.; New York, USA, 107-138.

Zobel, S., Kumpfmüller, J., Süssmuth, R.D., Schweder, T. (2015): Bacillus subtilis as heterologous host for the secretory production of the non-ribosomal cyclodepsipeptide enniatin, Applied Microbial Biotechnology, 99: 681-691. 


\title{
Chapter 2
}

\section{Enniatins production by $\boldsymbol{F}$. tricinctum on white bean medium and purification using flash chromatography}

\author{
Rosine G. Suchfort, Petr Karlovsky* \\ Molecular Phytopathology and Mycotoxin Research, Georg-August-University Göttingen, \\ Grisebachstr. 6, 37077 Göttingen, Germany \\ *Corresponding author: pkarlov@gwdg.de
}

\begin{abstract}
Strains $F$. tricinctum DSM 23357 and F. tricinctum $\mathrm{O} 32$ were examined for the production of enniatins A, A1, B and B1 on solid white bean (Phaseolus vulgaris ) (Wang et al., 2013) medium at $25^{\circ} \mathrm{C}$ for 30 days. A method was established for the quantification of enniatins content using HPLC UV-visible photodiode array detector (HPLC-DAD) with a reverse phase column, elution with a methanol-water gradient and detection by light absorption at $210 \mathrm{~nm}$. The enniatin content increased up to day 24. Total enniatin content of $F$. tricinctum O32 culture reached $614 \mathrm{mg} / \mathrm{kg}$, which was about two times more as compared to enniatin produced by $F$. tricinctum DSM 23357. F. tricinctum $\mathrm{O} 32$ was therefore used for the production of enniatins in white bean solid medium. Enniatins were extracted with ethyl acetate and the extract was fractionated using flash chromatography on a reverse phase column with a step gradient consisting of water-methanol mixtures. In total 92 fractions were collected and enniatins were detected using thin-layer chromatography (TLC) and HPLCDAD in fractions 50-74. Fractions containing enniatins (about $4.75 \mathrm{~g}$ per kg culture) were combined and further purified using recrystallization from methanol and water. $3.18 \mathrm{~g}$ enniatins containing about $77 \%$ enniatin $\mathrm{B}, 17 \%$ enniatin $\mathrm{B} 1,6 \%$ enniatin $\mathrm{A} 1$ and $0.3 \%$ enniatin A were obtained per $1 \mathrm{~kg}$ culture. The mixture of enniatin B, B1, A1 and A had a purity of $96.25 \%$.
\end{abstract}

Keywords: Enniatins, F. tricinctum, production, HPLC-DAD, flash chromatography. 


\section{Introduction}

The enniatin first discovered and known as enniatin A was isolated from the fungus Fusarium orthoceras by Gäumann et al. (1947). Enniatins are a group of cyclohexadepsipeptides composed of alternating residues of three $\mathrm{N}$-methyl amino acids, mostly valine, leucine and isoleucine depending on the variant, as well as three hydroxy acids, typically hydroxyisovaleric acid. The branched chain amino acids are linked by peptide and ester bonds. Enniatins are produced by several Fusarium species such as F. sambucinum (Audhya and Russell, 1974), F. avenaceum (Blais et al., 1992), F. oxysporum, F. acuminatum, $F$. arthrosporiodes, F. merismoides, F. tricinctum (Herrmann et al., 1996) and a few other genera like Verticillium hemipterigenum (Nilalonta et al., 2003). Twenty-nine variants of enniatins have been isolated and characterized; they differ from each other in their amino acid and hydroxy acid composition (Sy-Cordero et al., 2012). Figure 1 shows the general structure of enniatins as summarized by Sy-Cordero et al., 2012.

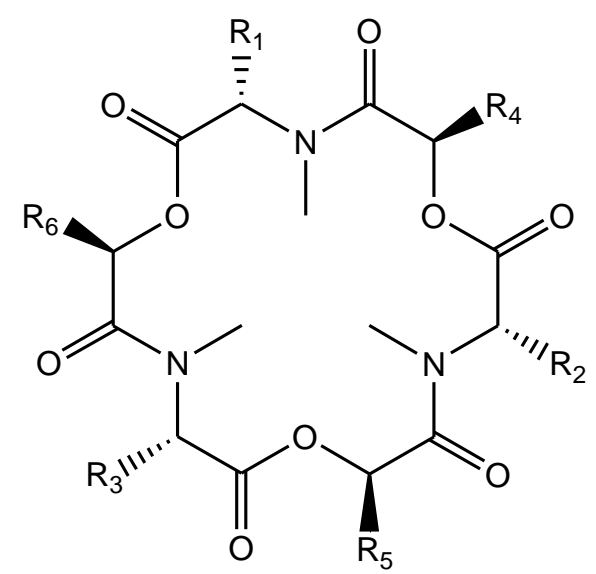

\section{Compound Radicals}

Enniatin A $\mathrm{R} 1=\mathrm{R} 2=\mathrm{R} 3=-\mathrm{CH}\left(\mathrm{CH}_{3}\right) \mathrm{CH}_{2} \mathrm{CH}_{3} ; \mathrm{R} 4=\mathrm{R} 5=\mathrm{R} 6=-\mathrm{CH}\left(\mathrm{CH}_{3}\right)_{2}$

Enniatin A1 $\mathrm{R} 1=\mathrm{R} 2=-\mathrm{CH}\left(\mathrm{CH}_{3}\right) \mathrm{CH}_{2} \mathrm{CH}_{3} ; \mathrm{R} 3=-\mathrm{CH}\left(\mathrm{CH}_{3}\right)_{2} ; \mathrm{R} 4=\mathrm{R} 5=\mathrm{R} 6=-\mathrm{CH}\left(\mathrm{CH}_{3}\right)_{2}$

Enniatin B $\mathrm{R} 1=\mathrm{R} 2=\mathrm{R} 3=-\mathrm{CH}\left(\mathrm{CH}_{3}\right)_{2} ; \mathrm{R} 4=\mathrm{R} 5=\mathrm{R} 6=-\mathrm{CH}\left(\mathrm{CH}_{3}\right)_{2}$

Enniatin B1 R1= R2 = $-\mathrm{CH}\left(\mathrm{CH}_{3}\right)_{2} ; \mathrm{R} 3=-\mathrm{CH}\left(\mathrm{CH}_{3}\right) \mathrm{CH}_{2} \mathrm{CH}_{3} ; \mathrm{R} 4=\mathrm{R} 5=\mathrm{R} 6=-\mathrm{CH}\left(\mathrm{CH}_{3}\right)_{2}$

Figure 1: General structure of enniatins, $\mathrm{R}_{1}$ to $\mathrm{R}_{6}$ could be sec-butyl, isobutyl, isopropyl, hydroxy-sec-butyl, ethyl or methyl radicals depending on the variant. 
In vitro production of enniatins using fungi has been reported in several studies using a variety of media including solid and chemically defined liquid media. Mainly analytical chromatographic and preparative chromatographic methods have been used for the detection, quantification and purification of enniatins. Early in 1974, Audhya and Russell demonstrated the production of enniatins in a semi-defined liquid culture by $F$. sambucinum, the process was light and temperature dependant, also the enrichment of cultures with carbon and nitrogen sources such as lactose, glycerol and tryptone supported enniatin production after detection and quantification with paper chromatography and spectrometry. The fermentation of $F$. avenaceum DAOM in liquid media resulted in the production of a mixture of enniatins containing predominantly enniatin A, followed by enniatins A1 and B1 (Blais et al., 1991). Wang et al. 2013 examined the production of F. tricinctum in seven solid media and in one liquid medium with variation in the supplementation of peptone or amino acid precursors. It was demonstrated that white bean solid media led to the highest enniatins yield in the growing condition chosen, after analysis using HPLC with UV-visible photodiode array detector. Solid corn was also used as substrate for enniatin production using $F$. tricinctum, the separation of the crude extract produced enniatins A, A1, B, B1, B4 and J (Cuomo et al., 2013). Furthermore, extraction of enniatins from crude liquid or solid media has been carried out using non polar solvents such as hexane and dichloromethane (Blais et al., 1991), ethyl acetate (Wang et al., 2013), chloroform (Madry et al., 1983) or moderate non polar solvent mixtures of water and methanol (Cuomo et al., 1991).

Biological activities of enniatins are mostly due to their ionophoric properties enabling them to translocate cations through bilayer membranes without the formation of membrane pores (Jestoi, 2008). Enniatins have shown antibiotic, insecticidal, anthelmintic and herbicidal activity in biological systems (Tomoda et al., 1992). A severe risk for humans associated with consumption of enniatins has been suggested by Cuomo et al. (2013) due to the induction of mitochondrial respiration resulting in the uncoupling of the oxidative phosphorylation.

Recently high enniatin concentrations in the range of several $\mathrm{mg}$ have been detected in food commodities with total enniatin (A, A1, B and B1) content up to $683.9 \mathrm{mg} / \mathrm{kg}$ in Tunisian sorghum (Oueslati et al., 2011), up to $1.1 \mathrm{mg} / \mathrm{kg}$ in commercial baby food samples (Juan et $a l ., 2014$ ), up to $0.10 \mathrm{mg} / \mathrm{kg}$ enniatins in Italian cereal and cereal products (Serrano et al., 2013), up to $1.8 \mathrm{mg} / \mathrm{kg}$ enniatins in nuts, dried fruits and dates from Spanish markets (Tolosa et al., 2013). 
Due to the multiple effects of enniatins encompassing the possible medical uses and the toxic effects to human resulting from high concentration in food commodities, we intended to purify enniatins for the purpose of searching some potential detoxifying microorganisms.

This study was carried out with two general objectives. First we intended to examine two $F$. tricinctum strains for enniatins (A, A1, B and B1) production on solid white bean media. Our specific objectives were: to establish a HPLC-DAD protocol for the detection and quantification of enniatin from fungal crude extract matrices after fermentation on solid white bean media; to quantify enniatins A, A1, B and B1 in the crude extract of fungi and establish a kinetics of enniatin production by the two strains over 30 days in order to find the best enniatins (A, A1, B and B1) producer strain. Secondly we intended to purify enniatins from solid white bean culture using the best enniatin (A, A1, B and B1) producer strain found from the previous part of the study. Enniatins were purified for further use in microorganisms screening with the capacity of detoxifying enniatins (as described in chapter 3 of this thesis). For the second part of this study, the specific objectives involved: the separations of enniatins in the crude extract using flash chromatography, the identification of positive fractions using TLC and HPLC-DAD; further purification of enniatins using recrystallization and finally the determination of the level of purity and the percentage of each enniatin variant in the purified mixture. 


\section{Material and methods}

\section{Part I}

\subsection{Examination of two Fusarium tricinctum strains for enniatin production}

\subsubsection{Microorganisms and solid culture}

F. tricinctum strains DSM 23357 and O32 used in this study originated from the Institute of Sugar Beet Research (Göttingen) and were isolated from sugar beet. To examine the production of enniatins (A, A1, B1 and A) by the two above mentioned Fusarium strains, three agar plugs ( $5 \mathrm{~mm}$ of diameter) of each of the two $F$. tricinctum strains grown on potato dextrose agar (PDA, from Merck, Darmstadt, Germany) plates for 5 days at $25^{\circ} \mathrm{C}$ were inoculated on white bean (Phaseolus vulgaris, Bio Bohnen, weiß, Ökologische Landwirtschaft DE-ÖKO- 001 Nicht EU- Landwirtschaft) solid medium. White bean solid medium was prepared by autoclaving $\left(120^{\circ} \mathrm{C}, 15 \mathrm{psi}, 15 \mathrm{~min}\right.$; Tuttnauer- Systec/ $\left.3870 \mathrm{EL}\right)$ $30 \mathrm{~g}$ white beans and $30 \mathrm{~mL}$ distilled water in $300 \mathrm{~mL}$ Erlenmeyer flasks. The cultures were incubated at $25^{\circ} \mathrm{C}$ (Schütt Lichtthermostat) for 30 days. Every 3 days, 3 flasks were collected and frozen at $-20^{\circ} \mathrm{C}$ until the extraction date. Three repetitions were made for each Fusarium strain and each time point.

\subsubsection{Extraction of enniatins and High Performance Liquid Chromatography analysis}

The total culture of each flask was ground in a mortar; two extractions were performed using $75 \mathrm{~mL}$ of EtOAc each by shaking using a shaker (Adolf Kühner AG Basel, Switzerland) at 175 RPM at room temperature for 14 hours and 6 hours respectively. The cultures were filtered under vacuum, the two extracts were mixed together and the solvent was initially evaporated in the rotary evaporator (Büchi 011, Switzerland, at $35^{\circ} \mathrm{C}$ ) equipped with a vacuum pump V-700 and vacuum controller V-855 (Büchi, Switzerland, at $150 \mathrm{mbar}$ ) and then in the SpeedVac (Christ RVC 2-25 CD plus, $35^{\circ} \mathrm{C}, 30 \mathrm{mbar}$ ) to obtain an oily extract.

High Performance Liquid Chromatography (HPLC) analysis of the ethyl acetate extracts was performed using a HPLC system equipped with a diode array detector (DAD) at $210 \mathrm{~nm}$. The HPLC instrument consisted of: a pump (PU-2085 Plus Semi-Micro HPLC Pump, Jasco Inc.), a detector (Varian Prostar PDA Detector), a sample injecting system (AS-2059 Autosampler, Jasco), a proportioning valve (LG-2080-04, 4-solvents), a degasser (DG-2080-54, 4solvents), a hardware interface between the computer and the system components (Jasco LC 
Net II / ADC), a C18 reversed phase column (Polaris C18-Ether, HPLC column, 180A, 5 $\mu \mathrm{m}, 100 \times 2.0 \mathrm{~mm}$, Agilent) in an oven (set at $40^{\circ} \mathrm{C}$ ). The separation was achieved using a gradient elution with distilled water (solvent A) and methanol (solvent B) at a flow rate of 0.2 $\mathrm{ml} / \mathrm{min}$. The composition of mobile phase was: solvent $\mathrm{A}: \mathrm{H}_{2} \mathrm{O}+7 \mathrm{mM}$ acetic acid $+5 \%$ ACN; solvent $\mathrm{B}: \mathrm{MeOH}+7 \mathrm{mM}$ acetic acid. The mobile phase gradient was as follows: $0-4$ $\min 70 \%$ B-98\% B; $4-13 \min 98 \%$ B-98\% B; $13-14 \min 98 \%$ B- $70 \%$ B $14-20$ min $70 \%$ B$70 \% \mathrm{~B}$.

Each extract was dissolved in $15 \mathrm{~mL}$ methanol; diluted 1:100 in methanol HPLC grade, filtered using micro-filters ( $25 \mathrm{~mm}, 0.20 \mu \mathrm{m}$, WICOM PERFECT-FLOW®) and $10 \mu \mathrm{L}$ were injected into the column. The enniatin variants (A, A1, B, B1) were identified according to comparison of the retention times and mass spectra with the standards, the concentrations were calculated with the use of the calibration curve of enniatin A, A1, B and B1 standards (from Gnomonia errabunda; $\geq 95 \%$ HPLC; Sigma-Aldrich Chemie GmbH Riedstr. 2D-89555 Steinheim). The quantification of enniatins was carried out on the basis of a comparison of peak areas with the calibration curves of standards.

\subsubsection{HPLC-MS confirmation of produced enniatins}

Confirmation of enniatin presence was carried out using a mass spectrometer system made of solvent delivery module (Varian Prostar 210), an autosampler (Varian Prostar 410), an ion trap $800 \mathrm{MS}$, an oven (Varian Model 510 Prostar). A C18 reversed phase column (Polaris C18-Ether, HPLC column, $180 \AA$, $5 \mu \mathrm{m}, 100$ x $2.0 \mathrm{~mm}$, Agilent) in an oven (temperature set at $40{ }^{\circ} \mathrm{C}$ ) was used. Liquid chromatography conditions were set up using a constant flow rate at $0.2 \mathrm{ml} / \mathrm{min}$ and the injection volume was $10 \mu \mathrm{L}$. Methanol and water $(7 \mathrm{mM}$ acetic acid + $0.01 \mathrm{mM}$ sodium acetate) were used as mobile phase in gradient elution consisting of a linear increase of methanol $70 \%$ to $98 \%$ for 4 minutes, then the methanol was maintained at $98 \%$ for 9 minutes, and decreased to $70 \%$ for 2 minutes and finally hold for 5 minutes at $70 \%$. The instrument was configured in the positive ionization mode. The injection volume was $10 \mu \mathrm{L}$. The sodium adduct ions 662.6; 676.6; 690.6; 704.5 were used as precursor ions for enniatin B, B1, A1 and A respectively. The daughter ions were: 549.4, 449.4 and 336.4 for enniatin B; 563.4, 463.4 and 336.4 for enniatin B1; 563.4, 463.4 and 350.5 for enniatin A1; 577.5 and 350.3 for enniatin A. 


\subsubsection{Statistical analysis}

Evaluation of the enniatins A, A1, B, B1 production by two $F$. Tricinctum strains DSM 23357 and $\mathrm{O} 32$ was done in three replicates for each fungi and each time point (3 days). The data was analyzed statistically using the software IBM SPSS for Macintosh version 22.0 (2013). Analysis of Variance (ANOVA) and post-hoc Tukey test in a completely randomized design were performed for the comparison of means of dependent variables, two tailed $\mathrm{P}$ values were considered as statistically significant on the level 0.05 .

\section{Part II}

\subsection{Large scale production of enniatins}

\subsubsection{Microorganism and culture}

According to the results obtained in the screening test, F. tricinctum $\mathrm{O} 32$ was retained for the large scale production of enniatin. The solid culture medium was prepared by autoclaving $\left(120^{\circ} \mathrm{C}, 15 \mathrm{psi}, 15 \mathrm{~min}\right.$; Tuttnauer- Systec/ $\left.3870 \mathrm{EL}\right) 200 \mathrm{~g}$ white beans (Phaseolus vulgaris) (Bio Bohnen, weiß, Ökologische Landwirtschaft DE-ÖKO- 001 Nicht EU-Landwirtschaft) and $200 \mathrm{~mL}$ distilled water in $1 \mathrm{~L}$ Erlenmeyer flasks. Five flasks were prepared. Twenty-five agar pieces $(5 \mathrm{~cm}$ diameter) of $F$. tricinctum $\mathrm{O} 32$ grown for 5 days on PDA (Merck, Darmstadt, Germany) plates at $25^{\circ} \mathrm{C}$ were inoculated under sterile conditions in one Erlenmeyer flask containing the solid white bean medium. The fungus was grown at $25^{\circ} \mathrm{C}$ for 30 days followed by extraction.

\subsubsection{Extraction and quantification of enniatins}

At time of harvest, the culture media containing mycelia were cut into small pieces with a knife inside the flask and $400 \mathrm{~mL}$ of ethyl acetate (EtOAc) were added to each flask. Enniatins were extracted on a shaker (Adolf Kühner AG Basel, Switzerland) at 175 RPM, at room temperature for 4 days and filtered under vacuum, the extraction was repeated a second time with the same volume of EtOAc for 3 days. The two filtrates were mixed together and the solvent was concentrated in a rotary evaporator (Büchi 011, Switzerland, at $35^{\circ} \mathrm{C}$ ) equipped with a vacuum pump V-700 and vacuum controller V-855 (Büchi, Switzerland, at 150 mbar) and finally dried overnight inside the SpeedVac (Christ RVC $2-25$ CD plus, $35^{\circ} \mathrm{C}$, 
30 mbar) to remove the residual solvent from the extract. The solvent evaporation resulted in an oily extract of $15 \mathrm{~mL}$ which was diluted with $2.5 \mathrm{~mL}$ methanol. A serial dilution was performed on a fraction of the extract and $10 \mu \mathrm{L}$ were injected in HPLC for the quantification of the enniatins in the crude extract as described previously (subheading 2.1.2).

\subsubsection{Preliminary purification of enniatins using flash chromatography}

The crude extract was purified with flash chromatography (Sepacore® Flash system $\mathrm{X} 10 / \mathrm{X} 50)$ using a constant flow rate of $25 \mathrm{ml} / \mathrm{min}$. The flash chromatography device consisted of: a pump Module C-601/C-605, disposable plastic cartridge of $31 \times 194 \mathrm{~mm}$ (Diameter $\times$ Length, Buchi Sepacore $\AA$ ) (loaded with $\mathrm{C} 18$, average pore size $60 \AA$, pore volume $0.8 \mathrm{~mL} / \mathrm{g}$, specific surface area $500 \mathrm{~m}^{2} / \mathrm{g}$, particle size $40-63 \mu \mathrm{m}$ ), a UV-Visible Detector C-640, a fraction collector C-660 and a computer with the SepacoreControl software. The mobile phase was composed of methanol and distilled water. The elution was performed with a step gradient starting with $100 \%$ water for $15 \mathrm{~min}$, then changed to $75 \%$ in $5 \mathrm{~min}$, held for $25 \mathrm{~min}$ at $75 \%$ and changed to $40 \%$ in $5 \mathrm{~min}$, the gradient was maintained at $40 \% \mathrm{MeOH}$ for $35 \mathrm{~min}$ before being reduced to $20 \%$ in $5 \mathrm{~min}$ and kept for $50 \mathrm{~min}$ at this concentration, methanol percentage was changed again to $0 \%$ for $5 \mathrm{~min}$ and kept at this concentration for $35 \mathrm{~min}$. The pressure was constant between 2-6 bars during the whole separation. The injection volume was $10 \mathrm{~mL}$ and the detection wavelength was $210 \mathrm{~nm}$. The collection time was 2 minutes. In total, 92 fractions were collected and aliquots of $1 \mathrm{~mL}$ of each was collected and transferred in a $1.5 \mathrm{~mL}$ Eppendorf tube, the elution solvent was evaporated in the SpeedVac (Christ RVC 2-25 CD plus, $35^{\circ} \mathrm{C}, 30 \mathrm{mbar}$ ) and the content of each Eppendorf tube was re-suspended in $1 \mathrm{~mL}$ methanol (HPLC grade) then diluted 1/10 and 1/1000 for checking the positive fractions using TLC and HPLC with UV-visible photodiode array detector (HPLC-DAD) respectively. 


\subsubsection{Screening of flash chromatography fractions}

TLC

The fractions eluted from the flash chromatography separation were monitored by TLC on normal phase silica gel plates $\left(40 \times 80 \mathrm{~mm}\right.$, ALUGRAM ${ }^{\circledR}$ Xtra SIL G/UV $\left.{ }_{254}\right)$ using DCM:MeOH (10:1; v/v) as mobile phase. The crude extract was diluted 1/100 and $1 \mu \mathrm{L}$ was spotted on the TLC plate, for the mixture of enniatin A, A1, B and B1 standards $(1 \mathrm{mg} / \mathrm{mL})$ and the flash fractions, $3 \mu \mathrm{L}$ and $1 \mu \mathrm{L}$ were spotted respectively. After development, the TLC plate was placed in a tank with iodine granules. The spots were visualized as brown spot and photographed under UV light at $254 \mathrm{~nm}$. The retention factor (Rf) of each fraction was calculated according to the formula below. In order to find the positive fractions, Rf value of the flash chromatography fractions was compared with that of enniatin standards. The weight of each positive fraction was recorded and they were mixed together.

$$
R f=\frac{\text { Distance travelled by the compound }}{\text { Distance travelled by the solvent front }}
$$

\section{HPLC-DAD}

HPLC-DAD analysis for the detection of positive fraction resulting from flash chromatography separation was performed using the protocol described previously (subheading 2.1.2). Enniatins identification was performed by comparing retention times and UV spectra of compounds present in each fraction and the pure standards.

\subsubsection{Purification of enniatin using recrystallization}

For purification of enniatins using recrystallization, $500 \mathrm{mg}$ of positive fractions were mixed with $3 \mathrm{~mL}$ heated methanol (about $50^{\circ} \mathrm{C}$ ) and allowed to cool down for 10 minutes at room temperature, then $1.5 \mathrm{~mL}$ distilled water was added. The enniatins contained in the mixtures were allowed to recrystallize at room temperature overnight and then kept on ice for 6 hours. The crystals were filtered under vacuum using a filter paper (Ederol quality, Nr. 75, Ø11cm) and rinsed with a cold mixture of methanol:water ( $\mathrm{v} / \mathrm{v} 2: 1$ ), then dried in a desiccator for 3 days. Recrystallization was carried out as described above to obtain a mixture of pure enniatins A, A1, B1 and B. The proportion of each enniatin variant in the recrystallized fractions was determined using HPLC-DAD as described previously (subheading 2.1.2). 
Purity check of the purified enniatin mixture was done using HPLC-DAD with the same conditions as mentioned previously (subheading 2.1.2) except that the mobile phase gradient used was as follows: 0-100 $\min 2 \% \mathrm{~B}-98 \% \mathrm{~B} ; 100-115 \min 98 \% \mathrm{~B}-98 \% \mathrm{~B} ; 115-120 \mathrm{~min}$ 98\% B-2\% B; 120-130 min 2\%B-2\% B. Solvent B was $\mathrm{MeOH}+7 \mathrm{mM}$ acetic acid. The level of purity was calculated by quantification of enniatins present in the mixture and comparison to the mass of the mixture used. 


\section{Results-Part I}

\subsection{Detection of enniatin standards and crude extract using HPLC}

Typical chromatograms of enniatin B, B1, A1 and A are presented in Figure 2, showing a clear separation of enniatin variants using HPLC with UV-visible detector. A separation and detection method was successfully developed for the separation of enniatins. The retention times were $8.13 \mathrm{~min}$; $8.45 \mathrm{~min}$; $8.85 \mathrm{~min}$; and $9.04 \mathrm{~min}$ for enniatins $\mathrm{B}, \mathrm{B} 1, \mathrm{~A} 1$ and $\mathrm{A}$ respectively. The optimal detection wavelength was found to be $210 \mathrm{~nm}$.
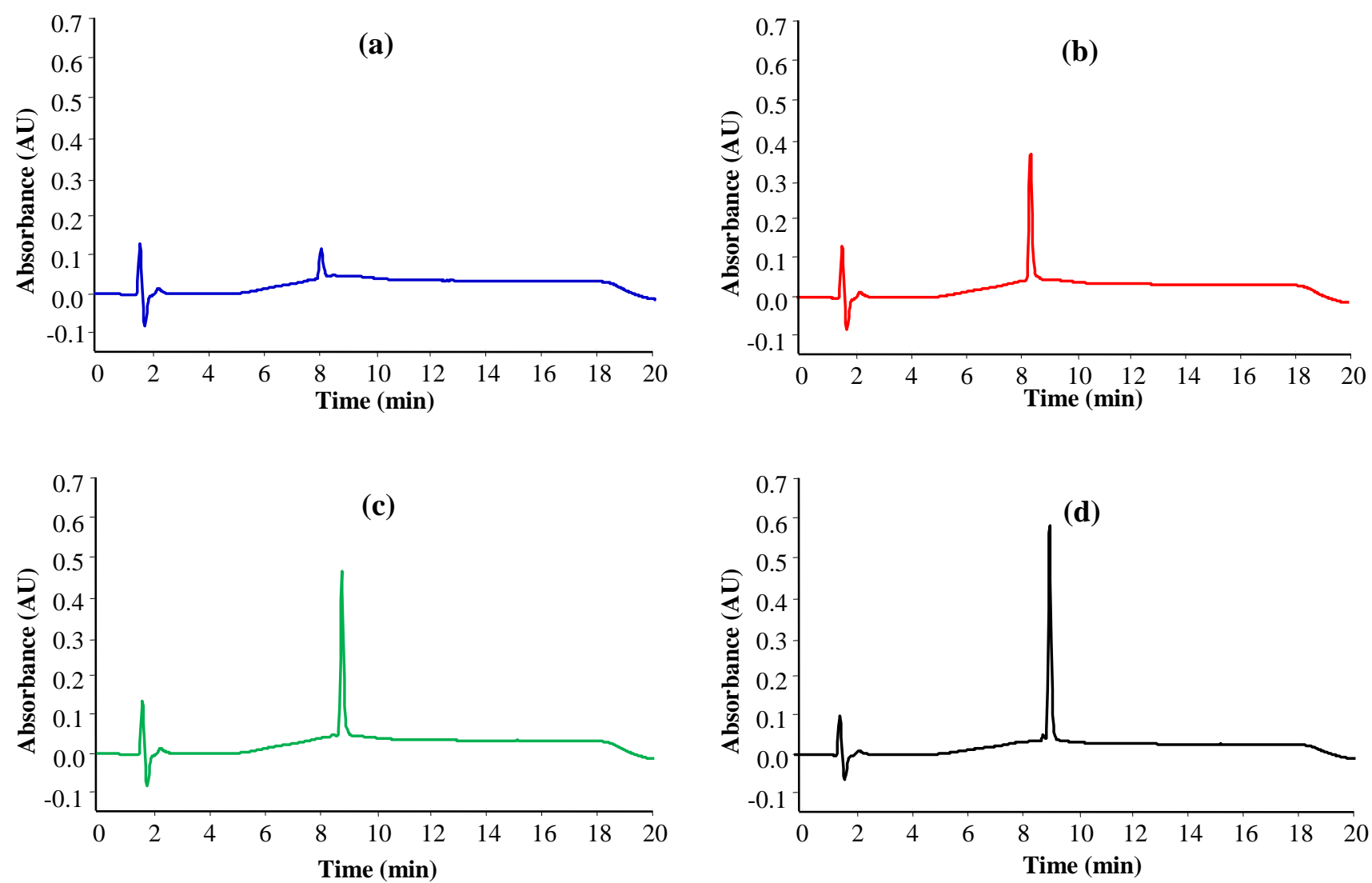

Figure 2: HPLC-UV Chromatograms of enniatin B (a), B1 (b), A1 (c) and A (d) standards at concentration 5 ppm. 
Figure 3 below shows a typical HPLC-UV chromatogram of ethyl acetate crude extract originating from fermentation of $F$. tricinctum $\mathrm{O} 32$ on solid white bean medium. The chromatogram patterns of crude extracts from both $F$. tricinctum strains used were similar. The fermentation of the two Fusarium strains on solid white bean medium produced enniatin $\mathrm{B}, \mathrm{B} 1, \mathrm{~A} 1$ and $\mathrm{A}$. The peaks were attributed to enniatin variants according to the retention times and UV spectra. The retention times were $8.13 \mathrm{~min}$; $8.53 \mathrm{~min} ; 8.85 \mathrm{~min}$; and $9.12 \mathrm{~min}$ for enniatin $\mathrm{B}, \mathrm{B} 1, \mathrm{~A} 1$ and A respectively.

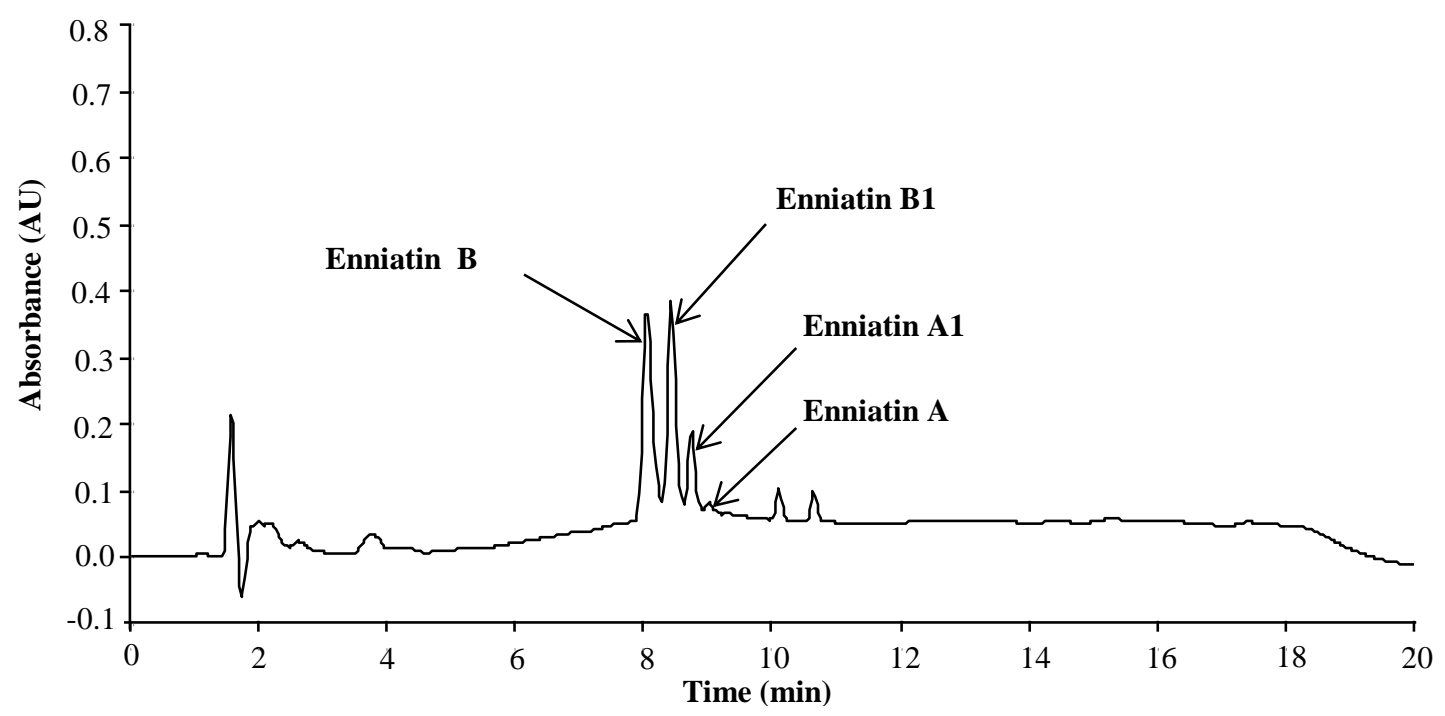

Figure 3: HPLC-UV chromatogram of a sample of $F$. tricinctum $\mathrm{O} 32$ after an incubation of 24 days grown on white beans and showing enniatins B, B1, A1 and A. 


\subsection{Calibration curves}

Calibration curves of enniatin B, B1, A1 and A are presented in Figure 4. Correlation coefficients ranging from 0.992 to 0.999 indicated linearity within the used concentration ranges.

(a)

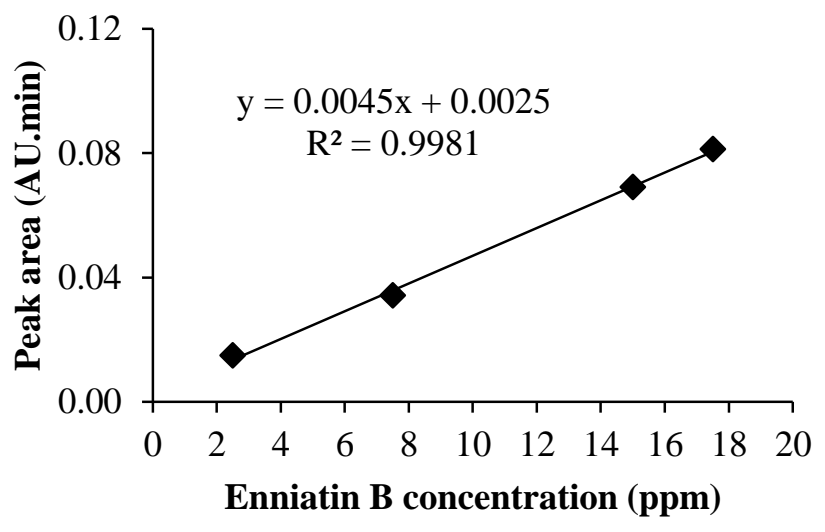

(c)

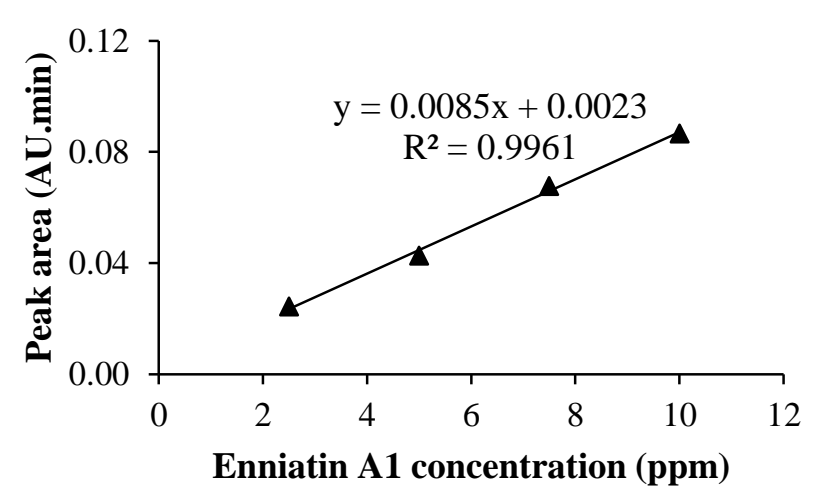

(b)

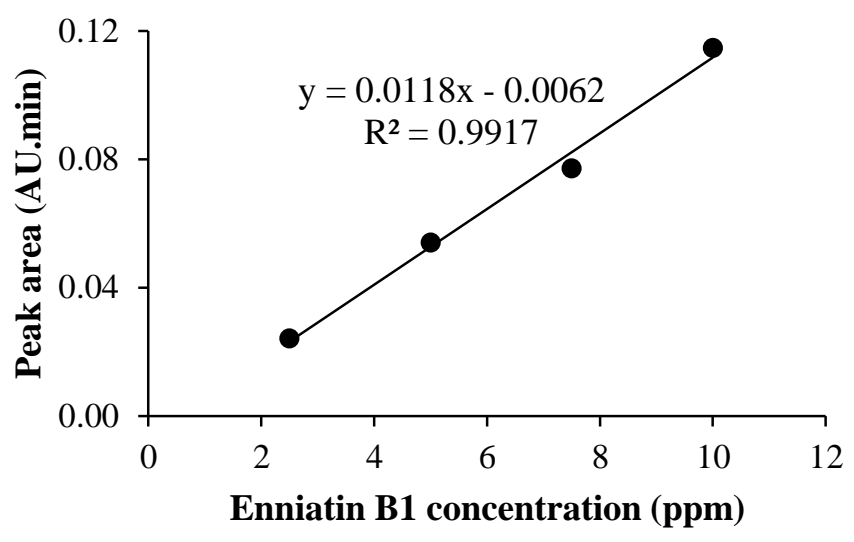

(d)

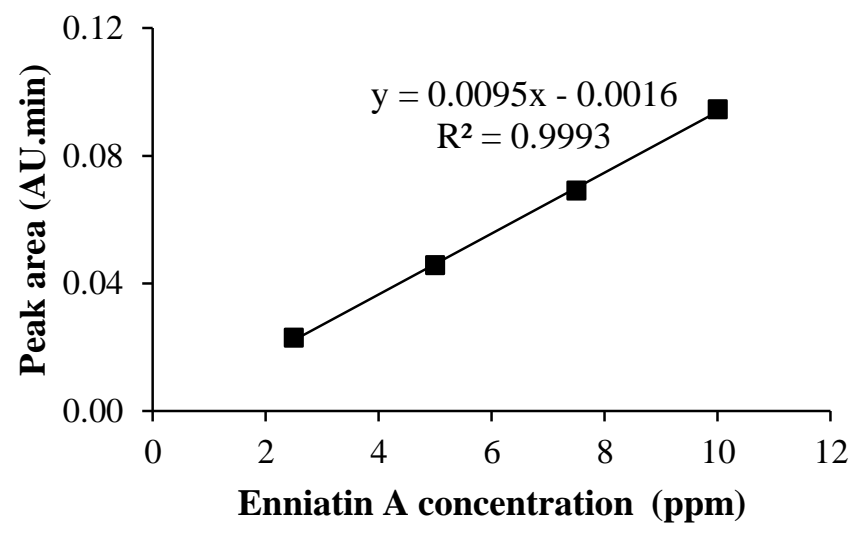

Figure 4: Calibration curves of enniatin standards B (a), B1 (b), A1 (c) and A (d).

\subsection{Assay validation}

Limit of detection and limit of quantification for enniatin A, A1, B1 and B were determined according to a signal/noise ratio of 3:1 and 10:1 respectively. The limit of detection was 1.23 ppm; 3.98 ppm; 1.78 ppm; 0.78 ppm for enniatins B, B1, A1 and A respectively. The limit of quantification was $4.091 \mathrm{ppm} ; 13.26 \mathrm{ppm} ; 5.94 \mathrm{ppm} ; 2.61 \mathrm{ppm}$ for enniatins B, B1, A1 and A respectively. The recoveries were calculated by diluting enniatin B, B1, A1 and A standards 
in pure methanol and quantifying them using HPLC-DAD. The mean recovery value from four replicates in concentrations ranging from 2.5 to $10 \mathrm{ppm}$ for enniatins $\mathrm{B} 1, \mathrm{~A} 1$ and $\mathrm{A}$ and 2.5- 17.5 for enniatin B was 100.47\%; 100.35\%; 99.97\%; $93.70 \%$ for enniatins B, B1, A1 and A respectively. All the samples were pretreated and the enniatin concentrations were within the measureable range. Values of the data analysis of calibration curve are summarized in Table 1.

Table 1: Construction of calibration curve and analysis of the data

\begin{tabular}{lcccc}
\hline Parameters & Enniatin B & Enniatin B1 & Enniatin A1 & Enniatin A \\
\hline Accuracy (\%) & $100.47 \pm 6.62$ & $100.35 \pm 4.11$ & $99.97 \pm 3.95$ & $93.70 \pm 3.46$ \\
Slope & 0.005 & 0.012 & 0.009 & 0.010 \\
Intercept & 0.003 & -0.006 & 0.001 & -0.002 \\
Correlation of determination $\mathbf{R}^{2}$ & 0.998 & 0.992 & 0.996 & 0.999 \\
Correlation coefficient R & 0.999 & 0.996 & 0.999 & 1.000 \\
Linearity range & $2.5-17.5$ & $2.5-10$ & $2.5-10$ & $2.5-10$ \\
Standard error of intercept & 0.002 & 0.005 & 0.003 & 0.001 \\
Standard deviation of intercept & 0.003 & 0.010 & 0.005 & 0.002 \\
LOD (ppm) & 2.211 & 2.661 & 1.772 & 0.776 \\
LOQ (ppm) & 7.369 & 8.870 & 5.906 & 2.585 \\
\hline
\end{tabular}

\subsection{Enniatin production by $F$. tricinctum strains $\mathrm{O32}$ and DSM 23357}

Production of enniatin A, A1, B1 and B by Fusarium strains O32 and DSM 23357 was monitored over a period of 30 days (Figure 5). In Appendix, Figure i shows the time course of enniatin production by two studied Fusarium strains expressed in ppm. The concentrations of enniatin B and A1 after 3 culture days were below the limit of detection and therefore were not considered in these calculations, for the same reason the concentrations of enniatin A were not considered at any time point. Total enniatin content mentioned refers to enniatin B, $\mathrm{B} 1$ and $\mathrm{A} 1$. The amount of total enniatin content produced by strain $\mathrm{O} 32$ over 30 days was significantly different $(\mathrm{P}<0.05)$ from the total enniatin content produced by strain DSM 23357 (statistics not shown). For Fusarium strains O32 and DSM 23357, the total enniatin concentration reached a maximum of $1228 \mathrm{mg} / \mathrm{L}$ and $959 \mathrm{mg} / \mathrm{L}$ respectively after 24 culture 
days corresponding to $614 \mathrm{~g} / \mathrm{kg}$ and $479 \mathrm{~g} / \mathrm{kg}$ white bean used respectively. Total enniatin production increased gradually until day 18 amounting $950 \mathrm{mg} / \mathrm{L}$ and $479 \mathrm{mg} / \mathrm{L}$ for strains O32 and DSM 23357 respectively and declined at day 21. For both strains, no significant difference $(\mathrm{P}>0.05)$ was detected among the values of the total enniatin concentrations at day 18 and day 21. The total enniatin concentrations peaked at day $24(1228 \mathrm{mg} / \mathrm{mL}, 959 \mathrm{mg} / \mathrm{mL}$ for strains O32 and DSM 23357 respectively) and declined to reach about $1095 \mathrm{mg} / \mathrm{L}$ and $674 \mathrm{mg} / \mathrm{L}$ for strains O32 and DSM 23357 respectively at day 30. However, no significant $(\mathrm{P}>0.05)$ difference was observed among total enniatin contents at day 24, day 27 and day 30 for strain O32. While no significant difference $(\mathrm{P}>0.05)$ was observed among the mean values of total enniatin content produced by strain $\mathrm{O} 32$ at day 24 and day 30, the mean values of total enniatin content produced by strain DSM 23357 at day 30 and day 24 were significantly different $(\mathrm{P}<0.05)$. Under the culture conditions set for this study and the Fusarium strains used, the optimal harvest time for enniatin A1, B and B1 production is between days 24 and 30. In general, throughout the 30 days, F. tricinctum strain $\mathrm{O} 32$ produced about two times more enniatins (B, B1 and A1) than the strain DSM 23357; therefore it was used for the large scale production of enniatins in the next step of this study.

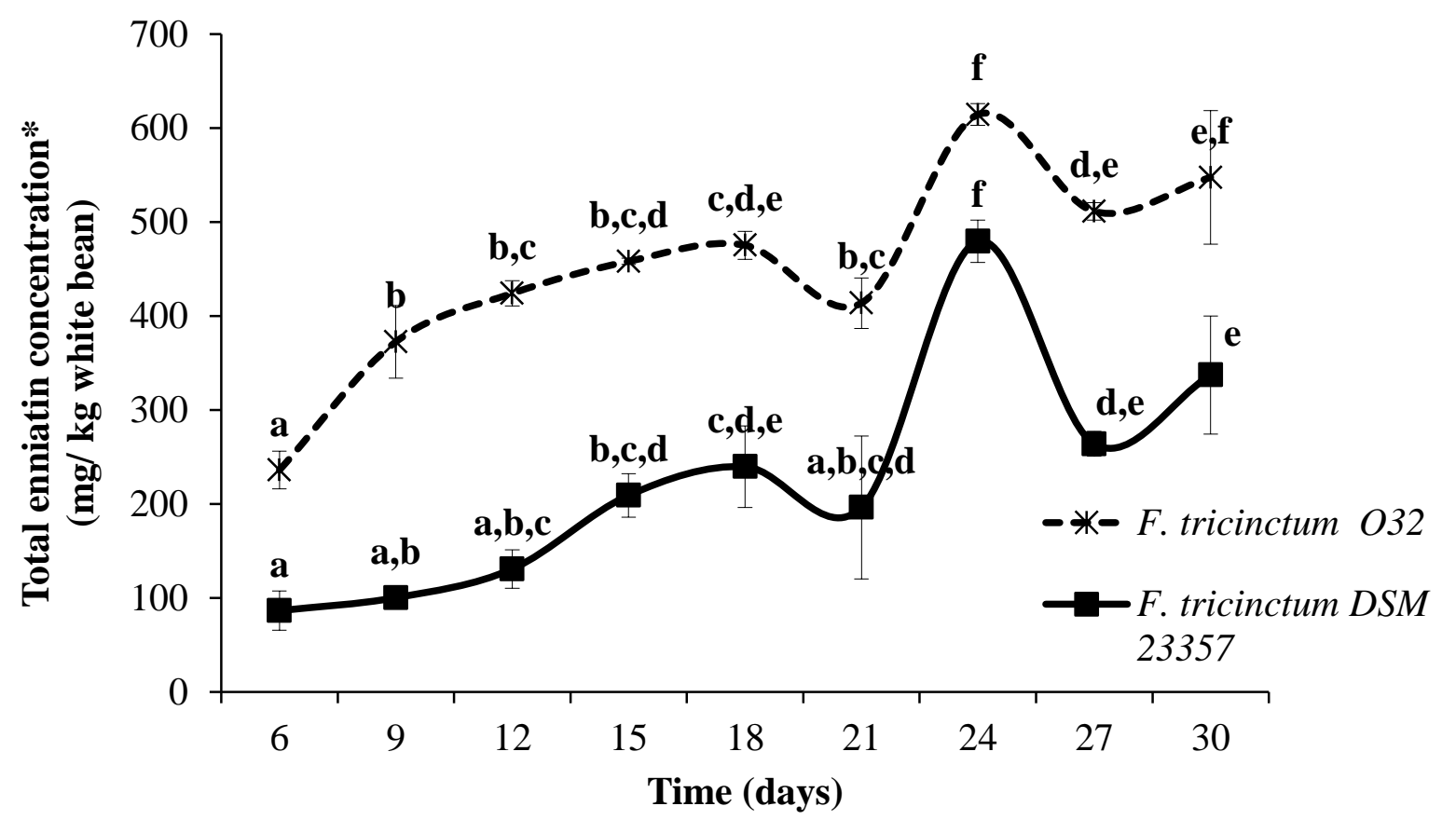

Figure 5: Time course of mean values of total enniatin (* refers to enniatins B, B1, and A) content in $\mathrm{mg} / \mathrm{kg}$ medium produced by $F$. tricinctum strains O32 and DSM 23357 on solid white bean medium from day 6 to day 30. a,b,c,d,e,f: Markers carrying the same letter within the same the line chart represent mean \pm Standard deviation statistically not different $P>0.05 ; n=3$. 


\subsection{HPLC-MS confirmation of produced enniatins}

The analysis of the ions seen in HPLC-MS showed $[\mathrm{M}+\mathrm{Na}]^{+}$ions at $\mathrm{m} / \mathrm{z}$ 662.6, 676.6, 690.6 and 704.5. The daughter ions obtained after fragmentation of each precursor ion are summarized in Table 2. Chromatograms of the above mentioned precursor ions and the mass spectra of daughter ions are shown in Figure 6. The retention times of the analyzed precursor ions were $4.31 \mathrm{~min}, 4.73 \mathrm{~min}, 5.02 \mathrm{~min}$ and 5.20 respectively.
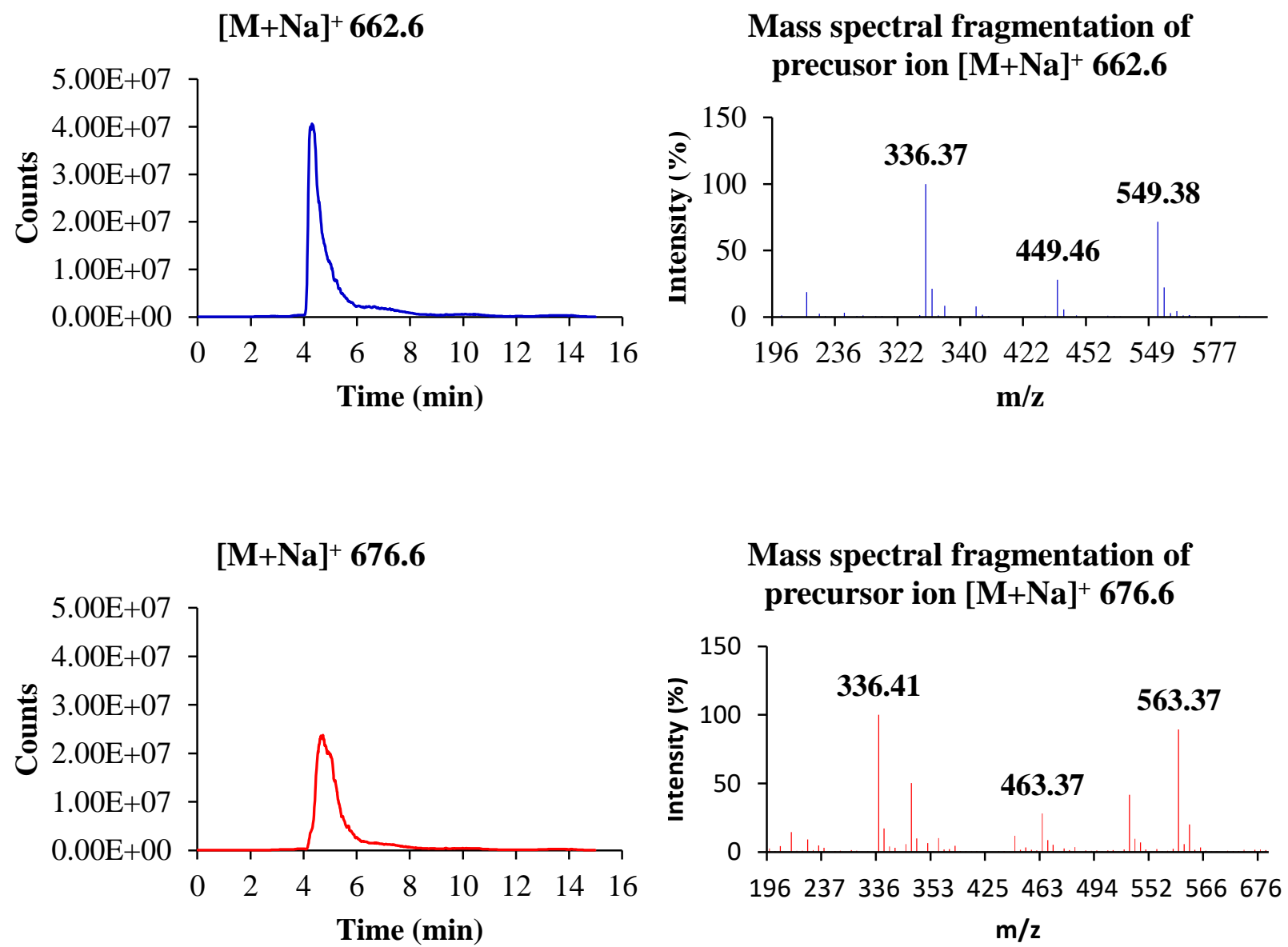

Figure 6: Precursor ions chromatograms and mass spectra of enniatins B, B1, A1 and A from the fungal extract of $F$. tricinctum $\mathrm{O} 32$ grown on solid white bean medium. 


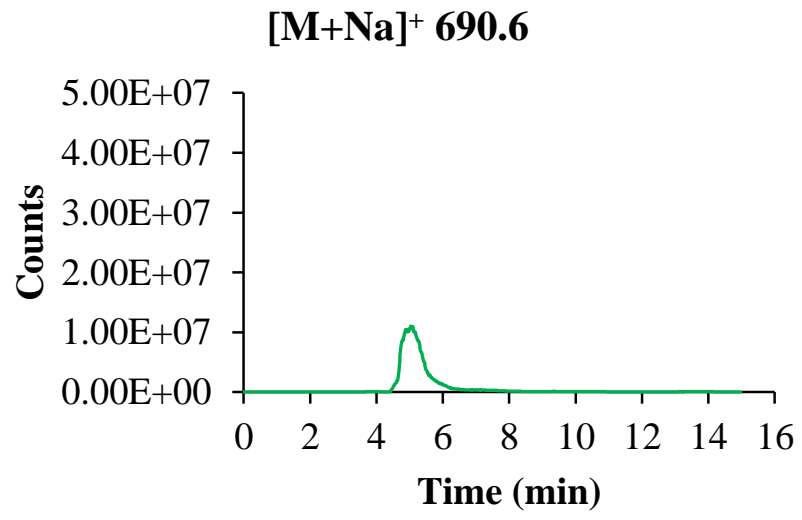

$[\mathrm{M}+\mathrm{Na}]^{+} \mathbf{7 0 4 . 5}$

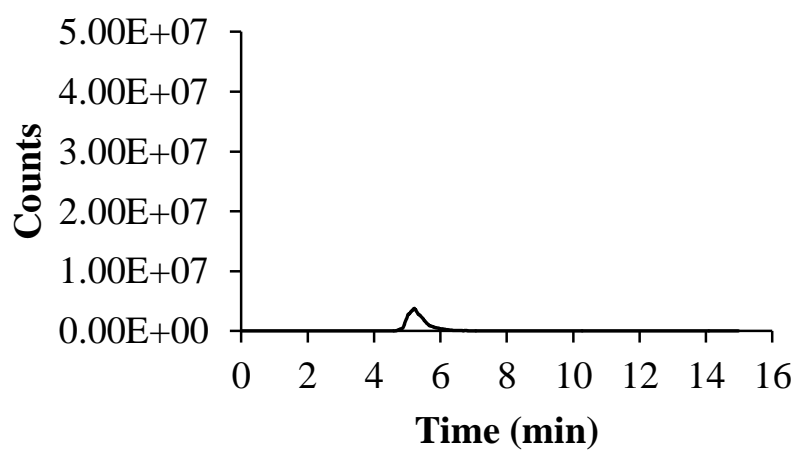

Mass spectral fragmentation of precusor ions $[\mathrm{M}+\mathrm{Na}]^{+} \mathbf{6 9 0 . 6}$

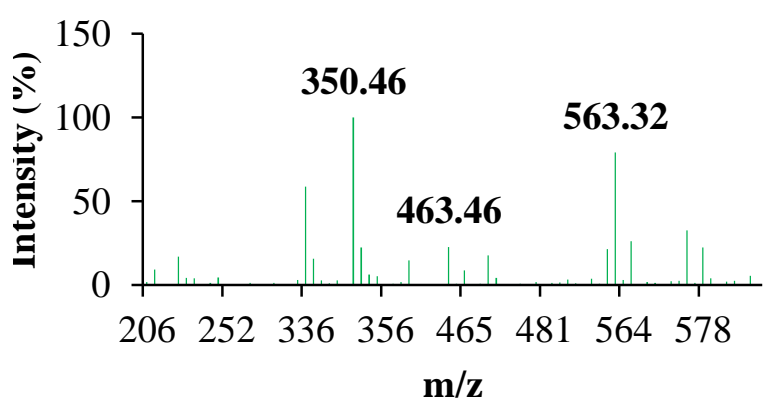

Mass spectral fragmentation of precursor ion $[\mathrm{M}+\mathrm{Na}]^{+} \mathbf{7 0 4 . 5}$

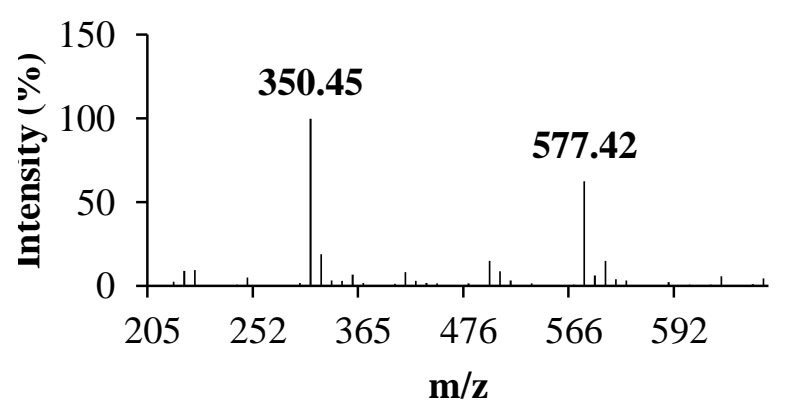

Figure 6 (cont.): Precursor ions chromatograms and mass spectra of enniatins B, B1, A1 and A from the fungal extract of $F$. tricinctum $\mathrm{O} 32$ grown on solid white bean medium.

Table 2: Daughter ions of the analyzed precursor ions

\begin{tabular}{lccc}
\hline Precursor ions $[\mathbf{M}+\mathbf{N a}]^{+}$ & \multicolumn{3}{c}{${\text { Daughter ions }[\mathbf{M}+\mathbf{N a}]^{+}}^{+}$} \\
\hline $\mathbf{6 6 2 . 6}$ & 549.38 & 449.46 & 336.37 \\
$\mathbf{6 7 6 . 6}$ & 563.37 & 463.37 & 336.41 \\
$\mathbf{6 9 0 . 6}$ & 563.32 & 463.46 & 350.46 \\
$\mathbf{7 0 4 . 5}$ & 577.42 & 350.45 & \\
\hline
\end{tabular}




\subsection{Relative proportion of enniatin variants}

In Figure 7 the mean of relative proportion of enniatin B, B1 and A1 content measured every three days from the $6^{\text {th }}$ to $30^{\text {th }}$ culture day is presented. Both F. tricinctum strains $\mathrm{O} 32$ and DSM 23357 show a similar pattern with regards to proportion of each enniatin variant in the cultures. The major variant was enniatin B accounting for more than $65 \%$ of the total enniatin content in the extract, followed by enniatin B1 and A1 accounting for more than $25 \%$ and less than $10 \%$ of the total enniatin content respectively.

The variation of percentage of each enniatin variant over the time was consistent in the crude extract of $F$. tricinctum O32. For enniatin B for example the percentage was between $63 \%$ and $66 \%$ during the study. In contrast to strain $\mathrm{O} 32$, the relative percentage of enniatin $\mathrm{B}$ produced by $F$. tricinctum DSM 23357 was not consistent and varied from 54\% to $79 \%$ during the study (data not shown).
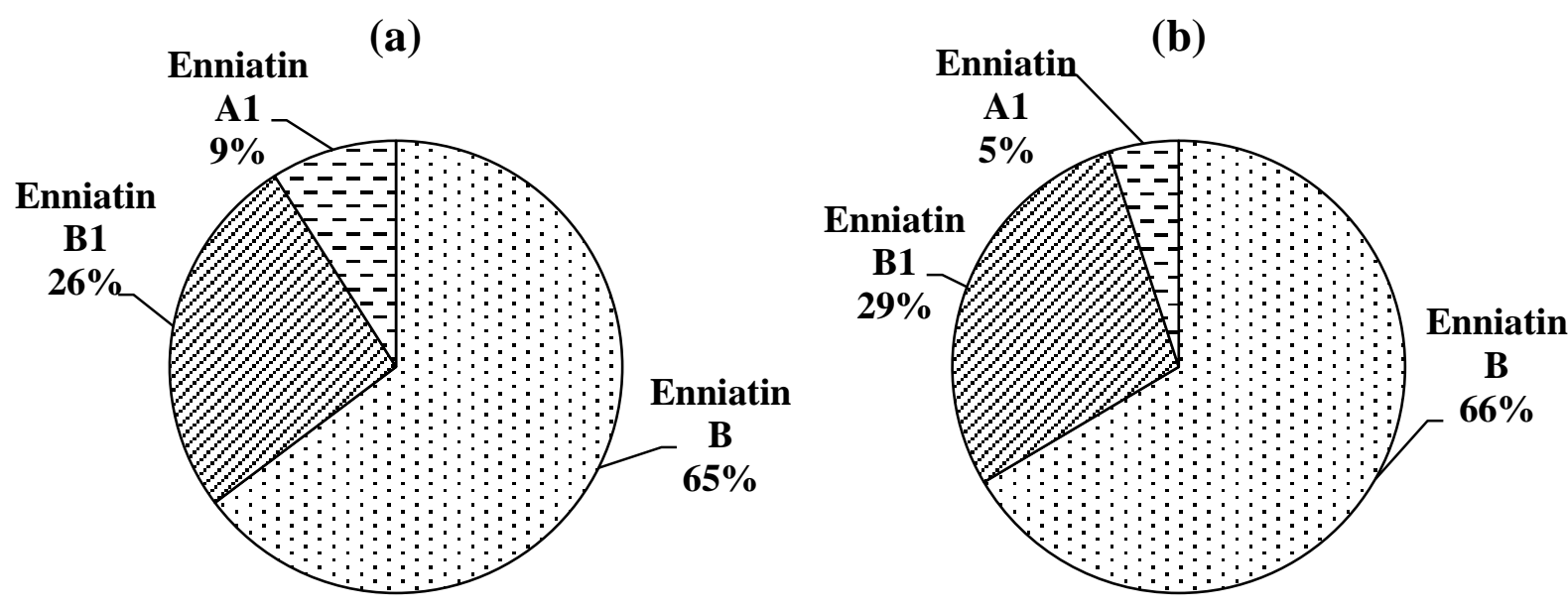

Figure 7: Relative percentage of enniatins (A1, B and B1) produced by strains $F$. tricinctum O32 (a) and F. tricinctum DSM 23357 (b) after 30 days on solid white bean medium. 


\section{Discussion-Part I}

\subsection{Enniatin production by $F$. tricinctum strains 032 and DSM 23357}

This part of the study was carried out in order to compare $F$. tricinctum strains O32 and DSM 23357 regarding their enniatin production on white bean medium over 30 days at $25^{\circ} \mathrm{C}$. Total enniatin produced during this study is comparable with data reported in other studies. For instance, Wang et al. (2013) reported enniatin A, A1, B1 and B yield of $1365 \mathrm{mg} / \mathrm{L}$ by $F$. tricinctum $\mathrm{AB} 470859.1$ cultured on white bean medium during 18 days, while in the present study a yield of enniatins A1, B1 and B reached $1228 \mathrm{mg} / \mathrm{L}$ and $959 \mathrm{mg} / \mathrm{L}$ after 24 days on solid white bean medium by $F$. tricinctum strains O32 and DSM 23357 respectively. Likewise, Meca et al. 2010 cultured F. tricinctum CECT 20150 on white corn solid medium during 30 days and recorded a yield of $1320 \mathrm{mg} / \mathrm{L}$ of enniatin A, A1, B, B1. Some earlier studies reported higher enniatin yields than the yield obtained during our study. For instance, Audhya and Russel (1973) reported a yield of 2000-3000 mg/L of enniatin A, B and C after 28 days fermentation of $F$. sambucinum Fuckel HLX316 in liquid medium supplemented with lactose and tryptone. In a study carried out by Madry et al. (1983), $5000 \mathrm{mg} / \mathrm{L}$ of enniatin A, A1, B, B1 were obtained after incubation of $F$. oxysporum ETH 1536/9 in a chemically defined liquid medium supplemented with amino acids for four days. In a further study, Herrmann et al. (1996) characterized thirteen Fusarium strains capable of producing enniatins in diverse media; of thirty-six tested strains, F. merismoides BBA 64329, F. lateririum BBA 65090 and F. sambucinum BBA 63933 exhibited the highest enniatin concentration of $500 \mathrm{mg} / \mathrm{L}, 400 \mathrm{mg} / \mathrm{L}$ and $350 \mathrm{mg} / \mathrm{L}$ respectively after being cultured in solid chemically defined medium FDM containing fructose for the first two strains and in solid FCM for the third strain during 6 days. These authors assessed enniatins as a group of compounds with the aid of photometric methods, the lack of specificity in the method could be the reason why higher yields were obtained during their study. Besides, Firáková et al. (2008) described a total enniatin $\mathrm{B}, \mathrm{B} 1$ and $\mathrm{G}$ of $350 \mathrm{mg} / \mathrm{L}$ after culturing $F$. dimerum in sabouraud maltose broth for 4 days.

The present study shows a divergence of the total enniatin content produced by two $F$. tricinctum strain O32 and DSM 23357 under the same conditions. In general, the strain O32 produced about two times more enniatins than strain DSM 23357. Similar findings have also been reported by Burmeister and Plattner (1987), after analyzing the production of enniatins by 13 strains of $F$. tricinctum grown on solid maize grit medium. A higher level of 
polimorphism of fifty-eight collection strains from twenty Fusarium strains were detected in a study reported by Stepien et al. (2013). This findings support the idea that, the enniatin synthetase (ESyn1) gene divergence might result in differences in toxin biosynthesis among strains of Fusarium of the same species.

Solid state fermentation (SSF) provides greater advantages than submerged fermentation, therefore is an alternative to submerged fermentation for the production of biologically relevant compounds such as antibiotics, proteins or enzymes (Mojsov, 2010). The technique of SSF is defined as the fermentation involving solids in absence (or near absence) of water, however, the substrate must possess enough moisture to support growth and metabolism of microorganisms (Pandey et al., 2001). This is the case during this study, several authors have reported high yield of enniatins from solid media such as solid corn or white bean (Burmeister and Plattner,1987; Meca et al., 2010; Wang et al., 2013).

\subsubsection{HPLC-MS confirmation of produced enniatins}

The presence of enniatin was confirmed by the presence of $[\mathrm{M}+\mathrm{Na}]^{+}$ions at $\mathrm{m} / \mathrm{z} 662,676.6$, 690 and 704.5 After HPLC-MS analysis of the samples. These compounds corresponded to $[\mathrm{M}+\mathrm{H}]^{+}$ions at m/z 640, 654, 668 and 682 also reported in several previous studies (Monti et al., 2000; Uhlig and Ivanova, 2004, Sørensen et al., 2008, Meca et al., 2010) which were identified as enniatin $\mathrm{B}, \mathrm{B} 1, \mathrm{~A} 1$ and $\mathrm{A}$ respectively. Also, the daughter ions $[\mathrm{M}+\mathrm{Na}]^{+}$for example at $\mathrm{m} / \mathrm{z} 549.38,563.37,563.32$ and 577.42 corresponded to daughter ions $[\mathrm{M}+\mathrm{H}]^{+}$at $\mathrm{m} / \mathrm{z}$ 527.3, 541.5, 541.2 and 55.4 reported by Cuomo et al. (2013).

\subsection{Relative proportion of enniatin variants produced by $F$. tricinctum 032 and DSM 23357}

In our study, enniatin B was the major constituent in the extracts of the two $F$. tricinctum strains, accounting for more than $65 \%$ of the total enniatin content followed by enniatin B1 and enniatin A1 present in about $25 \%$ and $10 \%$ respectively. The amount of enniatin A was not considered since the amounts were below the limit of detection. The high proportion of the other enniatin variants such as enniatin B would have been a risk for the HPLC equipment if the samples were concentrated to a greater degree. 
Several studies report enniatin B as the highest concentration followed by enniatin B1, A1 and $\mathrm{A}$ in food commodities such as wheat grains (Jestoi et al., 2004). The contamination level of the wheat correlated with the presence of $F$. arthrosporoides and F. tricinctum (Jestoi et al., 2004). Uhlig et al. (2006) investigated norwegian oat, barley and wheat samples from 2000 to 2002, enniatin B, B1, A1 and A percentages were respectively about $64 \%, 28 \% 8 \%$ in oat, $70 \%, 24 \%, 5 \%$ and $1 \%$ in barley and finally $79 \%, 18 \%, 2 \%$ and $1 \%$ in wheat. More recently, Wang et al. (2013) demonstrated a similar trend in samples of $F$. tricinctum AB 470859.1 cultured in solid white bean medium for 18 days. The proportions obtained were $43 \%, 38 \%, 12 \%$ and $7 \%$ for enniatin B, B1, A1 and A respectively. Although the proportions are different from ours, the same order of prevalence of enniatin B, B1, A1, A is reported in several studies. No clear explanation about the factors commanding the distribution of enniatin variants produced by Fusarium species are available in the literature at present. 


\section{Results-Part II}

\subsection{Enniatin purification}

Enniatins were purified at an earlier stage using flash chromatography and later on using recrystallization.

\subsubsection{Screening of positive fractions after flash chromatography separation TLC}

In total 92 fractions of $50 \mathrm{~mL}$ each were collected. Results of the TLC of each individual fraction using a mobile phase DCM:MeOH (10:1; v/v) and normal phase silica gel plates are presented in Figure 8. The retention factor of enniatin standards was $\mathrm{Rf}=0.52$. Fractions 50 to 74 contained principally compounds having the same retention factor as the enniatin standards as it can be seen on plates 5, 6 and 7. The positive fractions (50-74) resulting from the flash chromatography separation yielded $3.17 \mathrm{~g}$ product corresponding to $4.75 \mathrm{~g}$ product/kg white bean used. Figure ii in Appendix shows the positive fractions after solvent evaporation.

\section{HPLC-DAD}

Fractions containing enniatins resulting from the analysis of all 92 fractions collected from the flash chromatography separation were analyzed using HPLC-DAD; Figure 9 shows HPLC-UV chromatograms of some fractions namely fractions 50, 54, 55, 58, 61, 64, 70 and 73. Peaks with retention times of $8.08 \mathrm{~min}, 8.64 \mathrm{~min}, 9.09 \mathrm{~min}, 9.81 \mathrm{~min}$ corresponding to enniatin B, B1, A1 and A respectively can be observed. This confirmed a preliminary separation of enniatins from impurities in the fungal extract. 

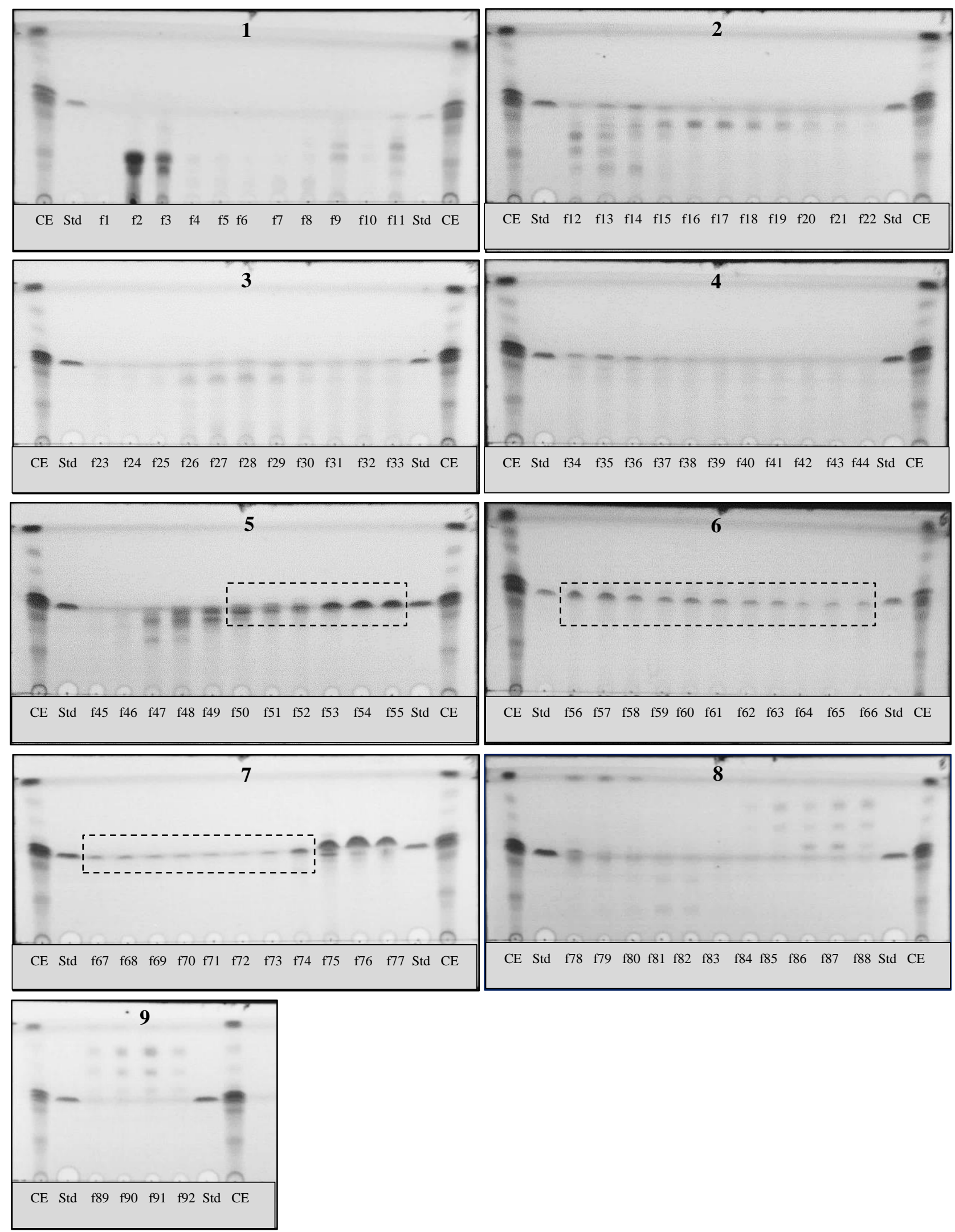

Figure 8: TLC plates of individual fractions resulting from flash chromatography separation; CE: crude extract, Std: enniatin standards, f: fraction. 

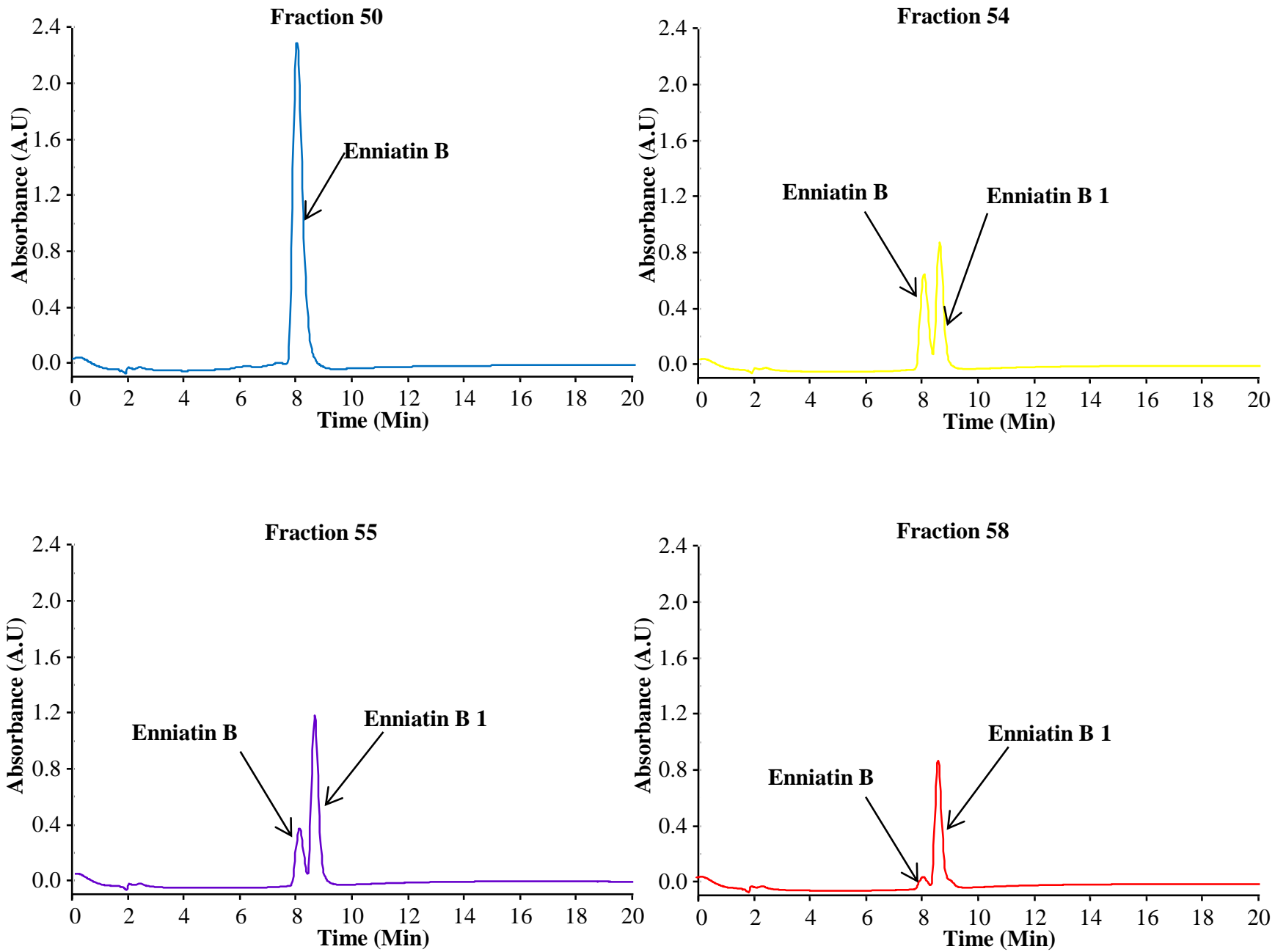

Figure 9: HPLC-UV chromatograms of fractions 50, 54, 55, 58, 61, 64, 70 and 73 resulting from the flash chromatography separation of enniatins in the ethyl acetate crude extract showing peaks corresponding to enniatin $\mathrm{B}, \mathrm{B} 1, \mathrm{~A} 1$ and $\mathrm{A}$. 

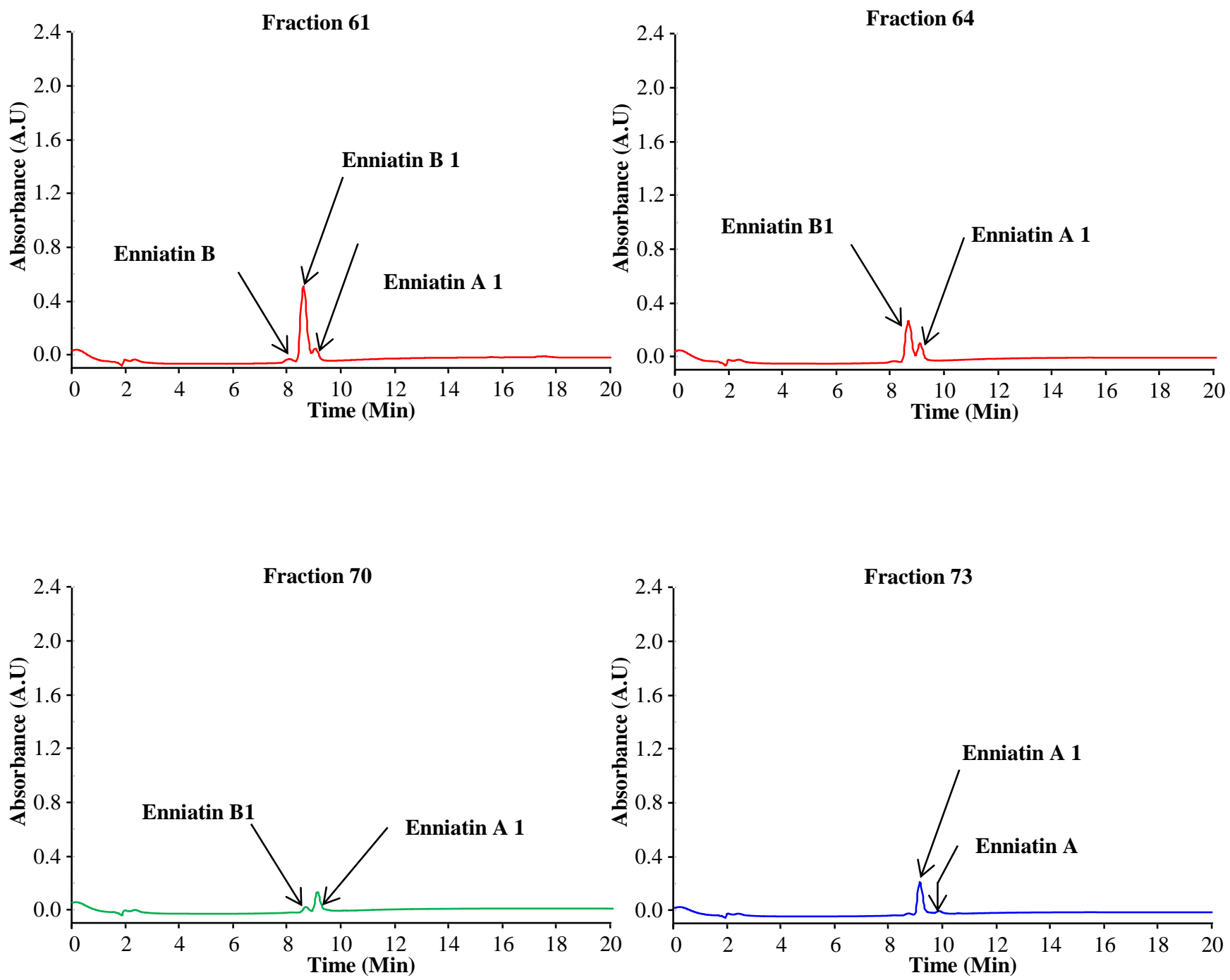

Figure 9 (cont.): HPLC-UV chromatograms of fractions 50, 54, 55, 58, 61, 64, 70 and 73 resulting from the flash chromatography separation of enniatins in the ethyl acetate crude extract showing peaks corresponding to enniatin B, B1, A1 and A.

\subsubsection{Recrystallization}

When subjected to recrystallization, the positive fractions yielded $2.12 \mathrm{~g}$ enniatins corresponding to 67\% yield (Figure iii in Appendix) containing about $77 \%$ enniatin B, 17\% enniatin B1, 6\% enniatin A1 and $0.3 \%$ enniatin A. This corresponds to $3.18 \mathrm{~g}$ enniatins $/ \mathrm{kg}$ white bean culture. The purity of enniatins B, B1, A1 and A in the recrystallized fraction was 96.25\%. Figure 10 shows HPLC-UV chromatograms of the crude extract and that of enniatins after recrystallization. The retention times of enniatins B, B1, A1 and A were 
$73.81 \mathrm{~min}, 75.97 \mathrm{~min}, 78.00 \mathrm{~min}$ and $79.81 \mathrm{~min}$ respectively. As it can be observed in Figure 10, chromatogram of recrystallized enniatins shows a more or less pure compound compared to that of the crude extract. Several peaks of compounds more hydrophilic than enniatins such as those eluting at $24.37 \mathrm{~min}, 40.55 \mathrm{~min}, 54.45 \mathrm{~min}$, and other compounds more hydrophobic than enniatins with the retention times $85.41 \mathrm{~min}, 88.80 \mathrm{~min}, 102.55 \mathrm{~min}$, $113.07 \mathrm{~min}$ and $115.37 \mathrm{~min}$ were no longer present in the purified fraction.

(a)
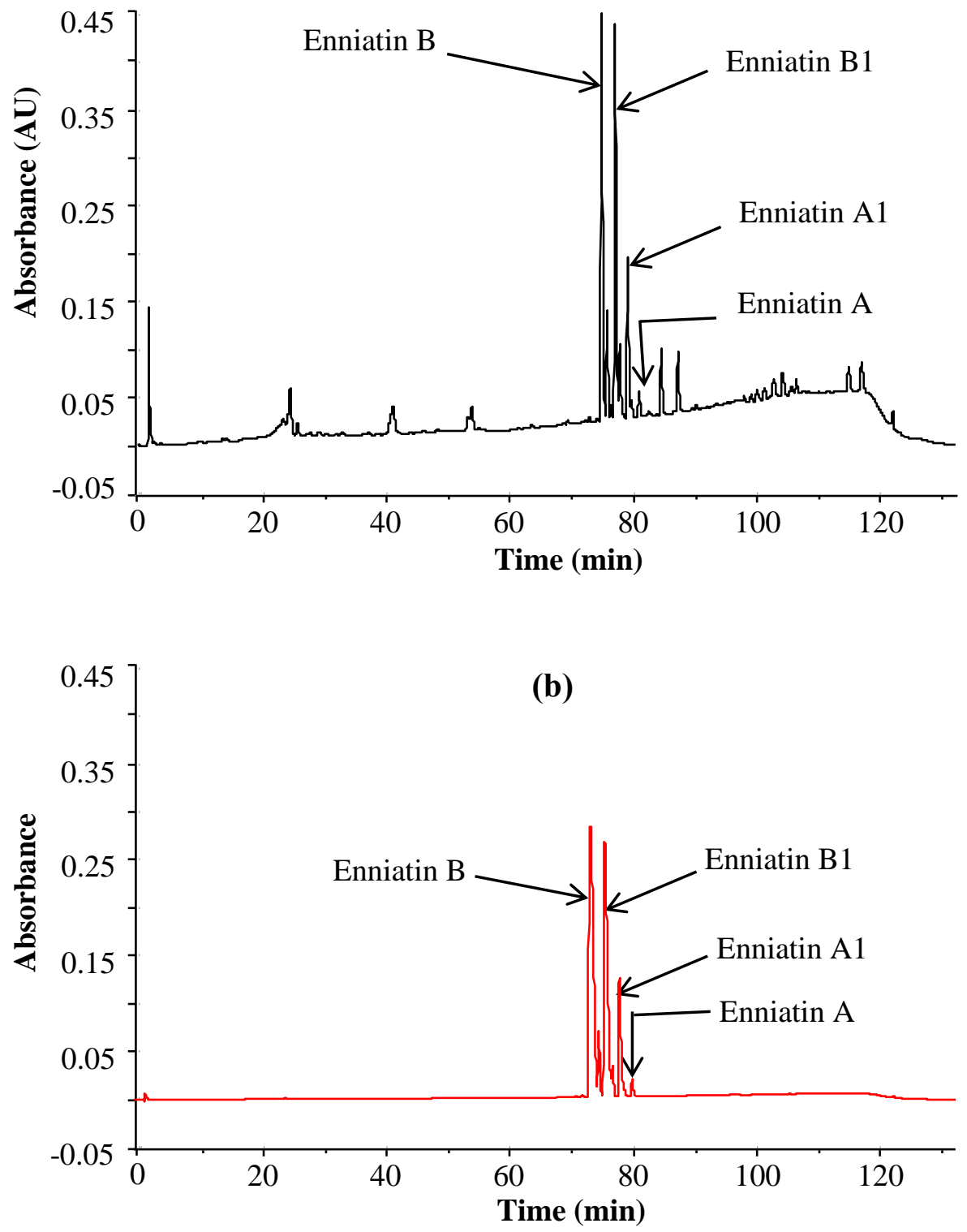

Figure 10: HPLC-UV chromatograms of the ethyl acetate crude extract of $F$. tricinctum $\mathrm{O} 32$ grown on solid white bean medium for 30 days (a) and that of enniatins purified by flash chromatography and recrystallization (b). 


\section{Discussion-Part II}

F. tricinctum $\mathrm{O} 32$ was grown on solid white bean medium for 30 days, then extracted using ethyl acetate, the crude extract was submitted to flash chromatography, followed by recrystallization in order to obtain a pure mixture of enniatins $\mathrm{A}, \mathrm{A} 1, \mathrm{~B}$ and $\mathrm{B} 1$. During this study our aim was to obtain a pure enniatin mixture and not individual enniatin variants for further use in detoxification as reported in chapter 3 of this thesis. This was done so as to get a scenario in which the relative percentage of enniatin variants is more or less similar to what is encountered in nature.

Previous studies have reported the purification of individual enniatin variants using combination of various chromatographic technics. For instance, Meca et al., 2010 purified enniatins B, B1, A and A1 from a fungal extract obtained after fermentation of F. tricinctum CECT 20150 on solid culture of corn during 30 days. The purification was achieved by low pressure liquid chromatography (LPLC) coupled to semi-preparative column liquid chromatography. Enniatins A (20 mg), A1 (46 mg), B1 (26 mg) and B (40 mg) were obtained from $100 \mathrm{~g}$ solid corn media. The fractions collected by the authors were analyzed using LCMS/MS. According to the report of Meca et al., 2010, during enniatin purification, enniatin A eluted before enniatin A1 using LC-UV, regarding this fact the results obtained by those authors were different to the one obtained during our study and also during studies carried out by several other authors (Sørensen et al., 2008; Wang et al., 2013). The quantity of total enniatins (including enniatin A, A1, B1 and B) purified by techniques used by Meca et al., (2010) corresponded to $0.132 \mathrm{~g} / \mathrm{kg}$ medium, whereas the yield obtained with the present method (using $F$. tricinctum $\mathrm{O} 32$, solid white bean medium, flash chromatography and recrystallization) was $3.2 \mathrm{~g} / \mathrm{kg}$ medium. Flash chromatography is a faster preparative chromatographic technique for the routine purification of complex mixtures ranging in several grams (Still et al., 1978). By comparison of the results obtained during the present study and those of Meca et al. (2010), flash chromatography is a fast and automated technique that is appropriate for the large scale purification of enniatins, the flash cartridge of $31 \times 194 \mathrm{~mm}$ (Diameter $\times$ Length, Buchi Sepacore ${ }^{\circledR}$, loaded with C18, average pore size 60 $\AA$, pore volume $0.8 \mathrm{~mL} / \mathrm{g}$, specific surface area $500 \mathrm{~m}^{2} / \mathrm{g}$, particle size $40-63 \mu \mathrm{m}$ ) used in our case was recommended for the separation of compounds ranging from 0.1-8 g. Thin Layer Chromatography was an easy and cheap method to find fractions containing mainly enniatins after flash chromatography separation. Moreover, precise insight on the composition of flash chromatography fractions was provided by HPLC-DAD showing that a separation of 
individual enniatin variants from each other and from other contaminants in the fungal extract was possible (although the fractions were intentionally mixed together). This again highlighted the efficacy of flash chromatography in purification of enniatins.

Recrystallization is a procedure for purifying compounds involving the separation of a small amount of contaminant from desired solid compound (Harwood and Moody, 1989), provided the desired compound has the capability to form crystals. During the present study, a double solvent recrystallization with methanol and water was used as a second step for the purification of enniatins. A mixture of enniatins B, B1, A1 and A crystals composed of about $77 \%$ enniatin $\mathrm{B}, 17 \%$ enniatin $\mathrm{B} 1,6 \%$ enniatin $\mathrm{A} 1$ and $0.3 \%$ was obtained. Burmeister and Plattner (1987) reported the production of enniatins B and B1 by $11 \mathrm{~F}$. tricinctum strains using MS/MS out of 13 tested, enniatins were purified using a combination of processes including methanol extract of the fungi cultured on white corn grit medium, a partitioning between chloroform and water, then a liquid extraction of the dried chloroform fraction using hexane and acetonitrile. Enniatins were then recrystallized by dissolution in boiling acetonitrile, followed by filtration. The compounds in the filtrate were allowed to recrystallize at $-18^{\circ} \mathrm{C}$ during 16 hours, after this the crystals were rinsed with hexane and acetonitrile. Enniatin were confirmed by MS, MS/MS and NMR. About 1-3 g/kg enniatin B and B1 were prurified from the culture of F. tricinctum NRRL 26430. In contratry to our study, no enniatin A and A1 was purified by these authors probably due to the many hydrophilic extractions performed in the purification process, given that $F$. tricinctum produced relatively low amount of enniatins A and A1 compared to enniatin B and B1. 


\section{Conclusion}

From this study, the following conclusions can be drawn:

The total enniatin B, B1 and A1 concentrations peaked at day 24 with $1228 \pm 23 \mathrm{mg} / \mathrm{mL}$, $959 \pm 45 \mathrm{mg} / \mathrm{mL}$ equivalent to $614 \pm 12$ and $479 \pm 23 \mathrm{mg} / \mathrm{kg}$ medium used for $F$. tricinctum strains O32 and DSM 23357 respectively.

The total enniatin B, B1 and A1 concentrations after 30 days were $1095 \pm 142 \mathrm{mg} / \mathrm{mL}$, $674 \pm 126 \mathrm{mg} / \mathrm{mL}$ equivalent to $547 \pm 71$ and $337 \pm 63 \mathrm{mg} / \mathrm{kg}$ medium used for $F$. tricinctum strains O32 and DSM 23357 respectively.

F. tricinctum strain $\mathrm{O} 32$ produced about two times more enniatin $\mathrm{B}, \mathrm{B} 1$ and $\mathrm{A} 1$ than $F$. tricinctum strain DSM 23357 under same conditions. Enniatin production under same conditions is therefore highly dependent on the strains.

According to the culture conditions set for this study and the Fusarium strains used, the optimal harvest time for enniatin A1, B and B1 production is between days 24 and 30 .

For both $F$. tricinctum strains used in our study, enniatin B was predominant in the fungal extract accounting of about $65 \%$ followed by enniatin B1 and A1 with relative percentages about $27 \%$ and $7 \%$ respectively.

The enniatin yield in the present study in comparison to previous studies shows that enniatin production varies according to species, culture condition and quantification method used.

The technique of purification of the fungal extract by flash chromatography coupled to recrystallization enabled a separation of enniatin $\mathrm{A}, \mathrm{A} 1, \mathrm{~B}$ and $\mathrm{B} 1$ from the impurities present in the extract, giving a mean purity of $96.25 \%$ of enniatin mixture. Criteria for determining the purity of the isolated enniatins were the quantification of enniatin variants using HPLCUV related to the mass of compound analyzed. Flash chromatography also enabled the separation of individual enniatin variants. This is a fast method to achieve large scale purification of enniatins from the complex fungal extracts.

The purified enniatin mixture contained about $77 \%$ of enniatin B, $17 \%$ of enniatin $\mathrm{B} 1,6 \%$ of enniatin $\mathrm{A} 1$ and $0.3 \%$ of enniatin A. 
A yield of about $3 \mathrm{~g}$ of enniatin mixture containing enniatin $\mathrm{B}, \mathrm{B} 1, \mathrm{~A} 1$ and $\mathrm{A}$ was obtained from $1 \mathrm{~kg}$ of white bean medium.

In this study an easy and efficient method for production and purification of enniatins $\mathrm{A}, \mathrm{A} 1$, $\mathrm{B}$ and B1 with high yield and purity has been proposed. 


\section{References}

Blais, L. A., Apsimon, J. W., Blackwell, B.A, Greenhalgh, R., Miller, J. D. (1992): Isolation and characterization of enniatins from Fusarium avenaceum, DAOM 196490, Canadian Journal of Chemistry, 70(5): 1281-1287.

Burmeister, H. R. and Plattner, R.D. (1987): Enniatin production by Fusarium tricinctum and its effects on germinating wheat seeds, Phytopathology, 77: 1483-1487.

Cuomo, V., Randazzo, A., Meca, G., Moretti, A., Cascone, A., Eriksson, O., Novellino, E., Ritieni, A. (2013): Production of enniatin A, A1, B, B1, B4, J1 by Fusarium tricinctum in solid corn culture: structural analysis and effect on mitochondrial respiration, Food chemistry 140: 784-793.

Firáková, S, Šturdíková, M., Liptaj, T., Prónayová, N., Bezáková, L., B. Proksa, B. (2008): Enniatins produced by Fusarium dimerum, an endophytic fungal strain, Pharmazie, 63: 539541.

Gäumann, E., Roth, S., Ettlinger, L., Plattner, P. A. \& Nager, U. (1947): Ionophore antibiotics produced by the fungus Fusarium orthoceras var. enniatum and other Fusaria, Experientia, 3: 202-203.

Harwood, L. M., Moody, C. J. (1989): Experimental organic chemistry: Principles and Practice, Oxford: Blackwell Scientific Publications, 127-132.

Herrmann, M., Zocher, R., Haese, A. (1996): Enniatin production by Fusarium strains and its effect on potato tuber tissue, Applied and environmental microbiology, 62 (2): 393-398.

Jestoi, M. (2008): Emerging Fusarium- mycotoxins fusaproliferin, beauvericin, enniatins and moliniformin- a review. Critical Reviews in Food Science and Nutrition, 48: 21-49.

Jestoi, M., Rokka, M., Yli- Mattila, T., Parikkas, P., Rizzo, A., Peltonen, K. (2004): Presence and concentrations of the Fusarium-related mycotoxins beauvericin, enniatins and moliniformin in Finnish grain samples, Food additives and contaminants, 21(8): 794-802.

Lukasz, S. and Agnieszka, W. (2013): Sequence divergence of the enniatin synthase gene in relation to production of beauvericin and enniatins in Fusarium species, Toxins, 5: 537-555. 
Meca, G., Ruiz, M.J., Soriano, J.M., Ritieni, A., Moretti, A., Font, G., Mañes J. (2010): Isolation and purification of enniatins $\mathrm{A}, \mathrm{A} 1, \mathrm{~B}, \mathrm{~B} 1$, produced by Fusarium tricinctum in solid culture, and cytotoxicity effects on Caco-2 cells, Toxicon, 56: 418-424.

Mojsov, K. (2010): Application of solid-state fermentation for cellulase enzyme production using Trichoderma viride, Perspectives of Innovations, Economics \& Business, 5(2), 108110.

Monti, S.M., Fogliano, V., Logrieco, A., Ferracane, R., Ritieni, A. (2000): Simultaneous determination of beauvericin, enniatins and fusaproliferin by high performance liquid chromatography, Journal of Agricultural and Food Chemistry, 48: 3317-3320.

Nilanonta, C., Isaka, M., Chanphen, R., Thong-orn, N., Tanticharoen, M., Thebtaranonth, Y. (2003): Unusual enniatins produced by insect pathogenic fungus Verticillium hemipterigenum: isolation and studies on precursor-directed biosynthesis, Tetrahedron, 59: 1015-1020.

Pandey, A., Soccol, C.R., Leo, J.A.R., Nigam, P. (2001): Solid-state fermentation in biotechnology, Asiatech Publishers, INc., New Delhi, 221.

Serrano, A.B., Meca, G., Font, G., Ferrer, E. (2013): Degradation study of enniatins by liquid chromatography-triple quadrupole linear ion trap mass spectrometry, Food Chemistry, 141: 4215-4225.

Sørensen, J.L., Nielsen, K.F., Rasmussen, P.H., Thrane, U. (2008): Development of a LCMS/MS method for the analysis of enniatins and beauvericin in whole fresh and in ensiled maize samples, Journal of Agricultural and Food Chemistry, 56(21): 10439-43.

Still, C.W., Kahn, M., Mitra, A. (1978): Rapid chromatographic technique for preparative separations with moderate resolution, Journal of Organic Chemistry, 43 (14): 2923-2925.

Sy-Cordero, A.A., Pearce, C.J., Oberlies, N.H. (2012): Revisiting the enniatins: a review of their isolation, biosynthesis, structure determination and biological activities, The Journal of Antibiotics, 65(11): 541-549. 
Tomoda, H., Huang, X.H. Cao, J., Nishida, H., Nagao, R., Okuda, S., et al. (1992): Inhibition of acyl-Coa: Cholesterol acyltransferase activity in cyclodepsipeptide antibiotics, Journal of Antibiotics, 45: 1626-1632.

Uhlig, S., Ivanova, I. (2004): Determination of beauvericin and four other enniatins in grain by liquid chromatography-mass spectrometry, Journal of Chromatography A, 2: 173-178.

Uhlig, S., Torp, M., Heier, B. (2006): Beauvericin and enniatin A, A1, B and B1 in Norwegian grain: a survey, Food chemistry, 94: 193-201.

Wang, J.P., Debbab, A., Hemphill, C.F., Proksch, P. (2013): Optimization of enniatin production by solid-phase fermentation of Fusarium tricinctum, Zeitschrift für Naturforschung C, 68(5-6): 223-230. 


\section{Appendix}

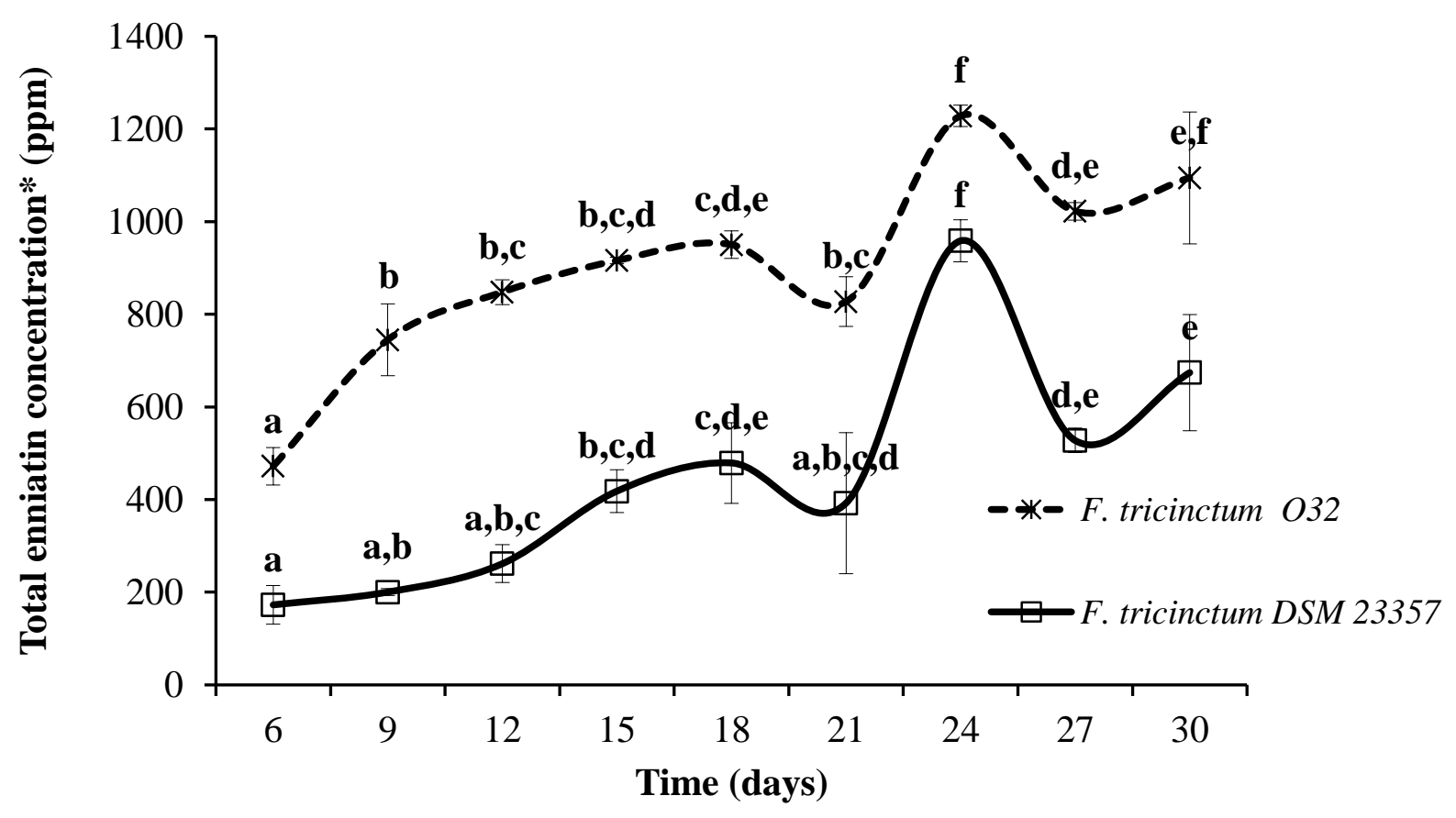

Figure i: Time course of means of total enniatin (B, B1 and A) content in ppm produced by F. tricinctum strains O32 and DSM 23357 on solid white bean medium from day 6 to day 30 . $a, b, c, d, e, f:$ Markers carrying the same letter within the same line chart represent mean \pm Standard deviation statistically not different $P>0.05 ; n=3$.

*Total enniatin concentration refers to enniatin B, B1 and Al content. 

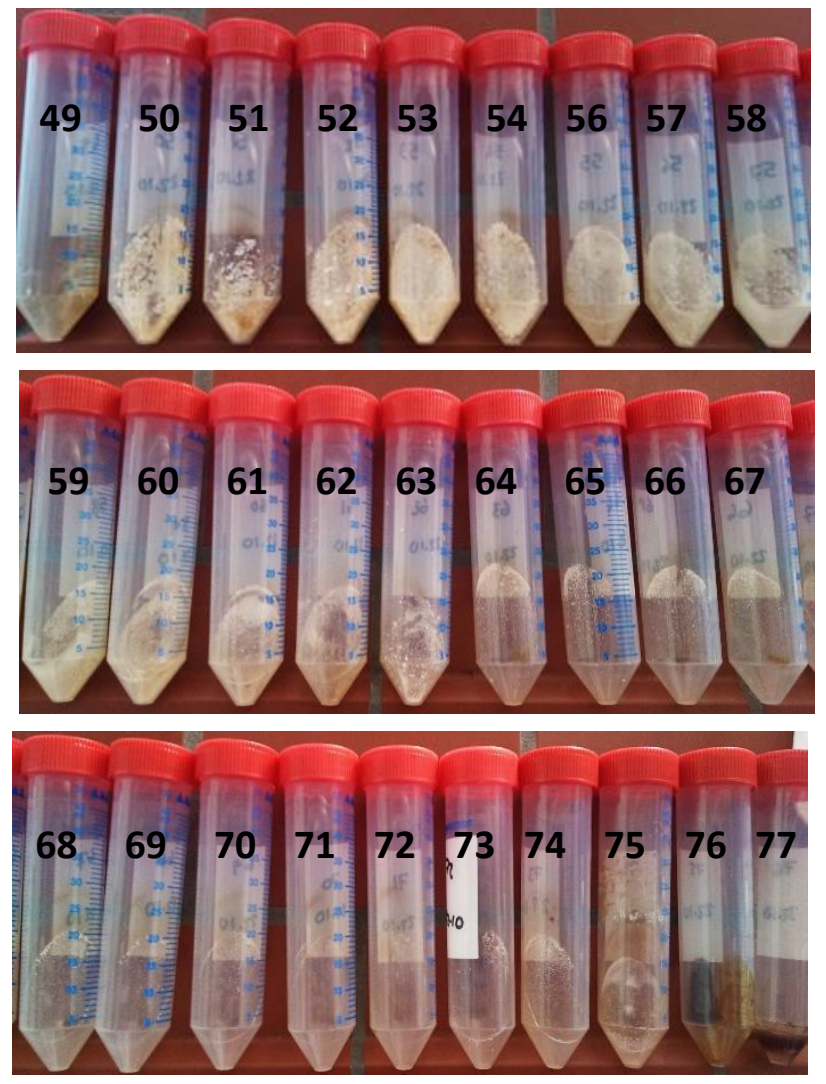

Figure ii: Fractions (including positive fractions, 50-74) resulting from flash chromatography separation after solvent evaporation in the SpeedVac.

The numbers of each fraction is written on the tubes.

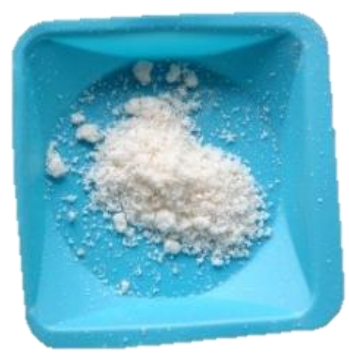

Figure iii: Dried enniatin crystals after recrystallization. 


\title{
Chapter 3
}

\section{Biological detoxification of enniatins and identification of degradation products using HPLC-MS}

\author{
Rosine G. Suchfort, Petr Karlovsky* \\ Molecular Phytopathology and Mycotoxin Research, Georg-August-University Göttingen, \\ Grisebachstr. 6, 37077 Göttingen, Germany \\ *Corresponding author: pkarlov@gwdg.de
}

\begin{abstract}
This study was conducted to isolate enniatin-degrading microorganisms (fungi, yeast or bacteria) from sources including soil, water, grains, cereal-based food and fruits. Wash fluid from these materials was mixed with a minimal medium containing enniatins (final concentration $1 \mathrm{mg} / \mathrm{ml}$ ) as unique carbon source and incubated for 7 days at $20^{\circ} \mathrm{C}$. The cultures were diluted 25-times in a minimal medium with enniatins and incubated for further 21 days. The cultures were plated on full agar media. A total of 114 isolates of bacteria and/or yeast-like microorganisms and 34 fungal isolates were obtained. These strains were inoculated individually into the minimal media and a minimal medium containing a limited amount of glucose and tryptone with enniatins as sole carbon source as described above. After 3 days (bacteria) or 5 days (fungi) enniatins were extracted from culture supernatants and analyzed for enniatin content by HPLC-DAD (RP chromatography, detection at $210 \mathrm{~nm}$ ). One bacterial strain and two fungal strains were able to transform enniatins into new products. Although enniatin content was reduced in most other cultures, new products were identified only in these three cultures using HPLC-DAD. Analysis including HPLC with fullscan-MS detection, differential metabolic profiling (noise reduction, chromatogram alignment, normalization) enabled the distinction of new signals in culture supernatants. Four enniatin degradation products resulting from the hydrolysis of presumably an ester bond in enniatin ring were identified.
\end{abstract}

Keywords: Enniatins, degradation, degradation products, HPLC-MS. 


\section{Introduction}

Enniatins are cyclic hexadepsipeptides composed of alternating D- $\alpha$ - hydroxy- isovaleric acid (HyLv) and several N-methylamino acid residues such as valine (Val), leucine (Leu) and isoleucine (Ile) (Jestoi, 2008). Some damaging effects on plants have been associated to enniatins, for example the water uptake of cells in tomato shoots is affected by enniatins, which leads to toxic wilt and necrosis (Gäumann et al., 1960). In an in vitro toxicity test a mixture of enniatins A, A1, B and B1 characterised by necrosis was also observed in potatoe tubers depending on the incubation time and the concentration of enniatins applied (Hermann et al., 1996). Moreover, a precise concentration of enniatins has been directly associated to a growth reduction of the wheat seed during germination, characterized by a higher inhibition of the root elongation compared to the leaf development (Burmeister and Plattner, 1987). The toxicity of enniatins is assumed to derive from their ionophoric properties and because they uncouple oxidative phosphorylation (Shemyakin et al., 1969). More recently, (Cuomo et al., 2013) showed an increase in the mitochondrial respiration leading to an uncoupling of the oxidative phosphorylation as a result of the flux of $\mathrm{K}^{+}$ions into the mitochondrial matrix was caused by enniatins $\mathrm{A}, \mathrm{A} 1, \mathrm{~B}, \mathrm{~B} 1$ and $\mathrm{J}$. The increase of the mitochondrial respiration suggested a severe risk for humans associated with consumption of enniatins. Besides these harmful effects of enniatins, they also possess some antibiotic properties which have been exploited in the production of an antibiotic known as fusafungine - a mixture of enniatin A, B and C - by Fusarium lateritium (Moffat, 1986). Furthermore, enniatins B, B1 and G were effective against some bacterial strains (Bacillus subtilis, Candida albicans, Trychosporom cutaneum and Cryptococcus neoformans) in agar diffusion tests by lowering the activity of the 12-lipoxygenase and therefore proved an antibiotic activity (Firákova et al., 2008). Another study highlights the fact that enniatins $J_{1}$ and $J_{3}$ could be potentially effective antibacterial agents against several pathogenic and lactic acid bacteria (Sebastià et al., 2011). Likewise, enniatin B induced the inhibition of microorganisms of some normally intestinal tract pathogens such as Escherichia coli, Salmonella enterica, Clostridium perfringens, Pseudomonas aeruginosa (Meca et al., 2011).

High concentrations of enniatins reaching $\mathrm{mg} / \mathrm{kg}$ have been detected in many food products including many cereals and cereal based products, but also in nuts and dried fruits (Jestoi et al., 2004; Yli-Mattila et al., 2008; Oueslati et al., 2011; Meca et al., 2011; Zinedine et al., 2011; Mahnine et al., 2011; Serrano et al., 2013; Tolosa et al., 2013; Juan et al., 2014). Due 
to lack of in vivo data of continual exposure to enniatins through food products, the chronic toxic effects of these compounds to humans are unknown (EFSA panel on contaminants in the food chain, 2014).

Biological detoxification is defined as the enzymatic degradation or transformation of toxins that lead to less toxic products (Bhatnagar, 1991). The enzymes involved in enzymatic degradation can originate from microorganisms, plants and animals. Unlike chemical and physical detoxification methods, biological detoxification has been poorly studied until the great advances in molecular and biotechnology techniques were developed. As mentioned by Karlovsky (1999) with the aid of such techniques it is possible to define the organisms, enzymes and genes responsible for the detoxification under specific conditions for further applications in the detoxification of agricultural systems, animal feed and food production.

Typical examples of the use of microorganisms for the biological detoxification of mycotoxins are reported in the literature. Early in 1966, Ciegler et al. demonstrated the ability of Flavobacterium aurantiacum to detoxify aflatoxin. Fusaric acid has been transformed by Penicillium spp., Aspergillus fumigatus and other fungi (Braun, 1960). The hydrolysis of the peptide bond of ochratoxin has been demonstrated by the activity of symbiotic microorganisms in the stomach of ruminant, leading to less toxicity of ochratoxin in ruminants (Hult et al., 1976). Duvick and Rood (2000) isolated bacterial strains Nocardia globulera or Rhodococcus fascians and Bacillus sphaericus leading to a reduction of beauvericin (structurally similar to enniatins) in vitro and in vivo. Deoxynivalenol (DON) the major toxin produced by Fusarium in grains- has been enzymatically detoxified in several experiments, among others Bacillus subtilis was demonstrated to remove $80 \%$ of DON after incubation with wheat and maize grains under anaerobic conditions (Cheng et al., 2010). Bacillus licheniformis CK1 reduced Zearalenone by $98 \%$ in Lysogeny broth and in corn kernels (Yi et al., 2011). A reduction of zearalenone of about $68 \%$ and $57 \%$ by Pseudomonas alcaliphila $\mathrm{TH}-\mathrm{C} 1$ and Pseudomonas plecoglossicida $\mathrm{TH}-\mathrm{L} 1$ respectively has been reported by Tan et al. (2014).

Considering that less research related to the biological detoxification of enniatins has been reported, the main aims of the present study were to screen microorganisms (fungi, bacteria or yeast) for the ablility to degrade enniatins into less toxic compounds and secondly to identify the degradation products. The specific objectives were to screen microorganisms based on their ability to grow on media containing enniatins as unique carbon source, to 
select microorganisms able to degrade enniatins. Then to find new products from the culture media in which the microorganisms have been incubated in comparison with non inoculated media by looking for new signals after HPLC-MS and then identify the masses of new compounds. Finally our objective was to elucidate the molecular formula of the degradation compounds and to predict the nature of the degradation reaction. 


\section{Material and methods}

\subsection{Selection of microorganisms for enniatin detoxification}

\subsubsection{Microorganism source}

The selection of microorganisms capable of growing on media containing enniatins as unique carbon source was performed using mixed cultures of several samples comprising foodstuff, soil and water from the local environment as summarized in Table 1. Grains and pasta material were crushed in Tissuelyser (Retsch MM300) with 2 wolfram carbide balls for 30 seconds; $0.3 \mathrm{~g}$ of each material was mixed with $2 \mathrm{~mL}$ sterile tap water in a $15 \mathrm{~mL}$ falcon tube. The same amount was taken for soil and fruit; the fruit was sliced into thin pieces, and mixed with $2 \mathrm{~mL}$ sterile tap water. No pretreatment was applied on water and juice samples. The samples used were grown on potato dextrose agar (PDA, Merck, Darmstadt, Germany) at $25^{\circ} \mathrm{C}$ for 7 days, an agar plug was cut out from each agar plate using a cork borer $(5 \mathrm{~mm}$ diameter) and mixed with $2 \mathrm{~mL}$ of sterile tap water. The tubes containing each material mixed with water were shaken at room temperature for two hours at 175 RPM, and then left for one hour to allow the material to settle, the fresh supernatant from each tube was collected inside sterile Eppendorf tubes. The mixed cultures were prepared in three replicates each.

Table 1: Overview of the sources from which microorganisms were obtained.

\begin{tabular}{lll}
\hline Microorganisms sources (S) & Origin \\
\hline Srain & Bio aus Liebe, Austria \\
S2: Summer wheat (var. Taifum) & KWS (Einbeck) \\
S3: Maize (var. Ronaldino) & KWS (Einbeck) \\
S4: Yellow soybean (var. Kanada) & Ewert, Göttingen \\
S5: Weed (Balium elarive) & Botanical gardens, \\
& Göttingen \\
S6: Mini maize (var. Gaspe flint) & KWS (Einbeck) \\
S7: Sorghum bicolor (var. "Tall & Leibniz Institute of plant \\
polish") & Genetics and Crop Plant \\
& Research in Gatersleben, \\
& Germany \\
S8: Rice genotype "Taichung Sen 10 & Africa Rice Center, \\
(TCS 10)" & Cotonou, Benin \\
S9: White beans & Bio Bohnen, weiß, \\
& Ökologische Landwirtschaft \\
& DE-ÖKO- 001 Nicht EU- \\
& Landwirtschaft \\
S10: Summer rape Brassica napus & Department of crop science, \\
var. napus Genom ACaacc & section plant pathology and \\
& crop protection (University \\
& of Göttingen, Germany) \\
\hline
\end{tabular}


Table 1 (cont.): Overview of the sources from which microorganisms were obtained.

\begin{tabular}{|c|c|c|}
\hline & Microorganisms sources (S) & Origin \\
\hline \multirow[t]{10}{*}{ Nut } & S11: Hazelnut (intact) & Trader Joe's \\
\hline & & Haselnusskerne ganz \\
\hline & & (Märsch Importhandels- \\
\hline & & GmbH, Ulm) \\
\hline & S12: Almond & Trader Joe's \\
\hline & & Hazelnusskerne ganz \\
\hline & & (Märsch Importhandels- \\
\hline & & GmbH, Ulm) \\
\hline & S13: Mung beans & $\begin{array}{l}\text { Uzbekistan (Mardin Food, } \\
\text { Denmark) }\end{array}$ \\
\hline & S14: Peanuts & Cameroon \\
\hline \multirow[t]{4}{*}{ Water } & S15: Water from lake & Göttingen \\
\hline & Wendebachstausee & \\
\hline & S16: Water from river Leine Kanal & Göttingen \\
\hline & S17: Water from lake Kiessee & Göttingen \\
\hline \multirow[t]{4}{*}{ Soil } & S18: Soil from a wheat field & $\begin{array}{l}\text { Wendebachstausee } \\
\text { Göttingen }\end{array}$ \\
\hline & S19: Garden soil & Geismar Göttingen \\
\hline & S20: Soil from a wheat Field & Weende Nord Göttingen \\
\hline & S21: Soil from a maize field & Geismar Göttingen \\
\hline \multirow[t]{6}{*}{ Juice } & S22: Apple juice & $\begin{array}{l}\text { Apfelsaft,Vitafit, Moers, } \\
\text { Deutschland }\end{array}$ \\
\hline & S23: Orange juice & Hohes C Eckes- Granini \\
\hline & & Deutschland GmbH \\
\hline & S24: Tomato juice & A. Dohrn \& A. Timm \\
\hline & & GmbH \& Co. KG, \\
\hline & & Diedersdorf, Deutschland \\
\hline \multirow[t]{5}{*}{ Pasta } & S25: Chinese noddle & $\begin{array}{l}\text { Kridsada Food CO., LTD, } \\
\text { Bangkok, Thailand }\end{array}$ \\
\hline & S26: Noodle Penne & $\begin{array}{l}\text { Erfurter Teigwaren GmbH, } \\
\text { Erfurt, Deutschland }\end{array}$ \\
\hline & S27: Vegetarian Noodle Vietnam & $\begin{array}{l}\text { Hoang Tuan Food PTE, } \\
\text { Vietnam }\end{array}$ \\
\hline & S28: Noodle Echt Schwäbische & Schätzle, Gesellschaft für \\
\hline & Frischei-Nudeln & $\begin{array}{l}\text { Nahrungsmittel-Herstellung } \\
\text { und Vertrieb mgH, }\end{array}$ \\
\hline Strains & S29: 5 Fungal strains from forest & Göttingen \\
\hline \multirow[t]{4}{*}{ Fruits } & 30: Plums & Göttingen \\
\hline & S31: Raisins & Tafeltrauben, Thompson \\
\hline & & Seedless, Labros Giorgos \\
\hline & & A.S. Griechenland \\
\hline
\end{tabular}




\subsubsection{Selection of microorganisms from mixed culture}

To select microorganisms able to grow on media containing enniatins as the unique carbon source, the following procedure was used. $10 \%(\mathrm{v} / \mathrm{v})$ solution of minimal medium (MM) (pH 5.5 and $\mathrm{pH}$ 7.2) containing enniatins was prepared using a stock solution of enniatins (10 mg/ $\mathrm{mL}$ ) in order to get a culture medium containing $1 \mathrm{mg} / \mathrm{mL}$ of enniatins. $100 \mu \mathrm{L}$ of the mixture MM/enniatins were put in each well of a microtiter plate (96 well plates). To each well $4 \mu \mathrm{L}$ of fresh supernatant from each source of microorganisms was added, $4 \mu \mathrm{L}$ sterile water was added in the control wells as represented in Table 2. The microtiter plates were incubated at $20^{\circ} \mathrm{C}$ for one week. A 10 times dilution was made from the content of each well and $4 \mu \mathrm{L}$ of each dilution was transferred to a new well containing $100 \mu \mathrm{L}$ of MM/enniatins $(1 \mathrm{mg} / \mathrm{mL})$. The new microtiter plates were incubated for three weeks at $20^{\circ} \mathrm{C}$. Every week, the wells were checked for the disappearance of enniatin crystals and the presence of a colloidal suspension or mycelium using a binocular microscope. Agar plates containing a full medium (FM) having the same $\mathrm{pH}$ either $\mathrm{pH} 5.5$ or 7.2 (depending on the $\mathrm{pH}$ of the MM used for microorganism screening) were used. After incubation, the content of each well was streaked onto the agar plates to obtain single bacterial colonies. Fungi were isolated using the dilution series and the dilution plating method.

The enniatins originated from the process described in chapter 2 of this thesis, the mixture contained about $77 \%$ enniatin B, $17 \%$ enniatin B1, $6 \%$ enniatin A1 and $0.3 \%$ enniatin A had a purity of $96.25 \%$.

The composition of $\mathrm{MM}$ is as follows: $0.8 \mathrm{~g} \mathrm{~K}_{2} \mathrm{HPO}_{4}, 0.2 \mathrm{~g} \mathrm{KH}_{2} \mathrm{PO}_{4}, 0.2 \mathrm{~g} \mathrm{MgSO}_{4}, 1.5 \mathrm{~g}$ $\mathrm{NH}_{4} \mathrm{Cl}, 0.001 \mathrm{~g} \mathrm{CaCl}_{2}, 0.001 \mathrm{~g} \mathrm{FeCl}_{3}$, and $2.0 \mathrm{ml}$ trace element solution (TS2) per liter medium (Völkl et al., 2004). The TS2 contained per liter medium: $100 \mathrm{mg} \mathrm{ZnSO} \mathrm{Zn}_{4} 7 \mathrm{H}_{2} \mathrm{O}, 20$ $\mathrm{mg} \mathrm{Fe}-\mathrm{SO}_{4} \cdot 4 \mathrm{H}_{2} \mathrm{O}, 30 \mathrm{mg} \mathrm{MnCl}_{2} .4 \mathrm{H}_{2} \mathrm{O}, 10 \mathrm{mg} \mathrm{CuCl} 2.2 \mathrm{H} 2 \mathrm{O}, 300 \mathrm{mg} \mathrm{H}_{3} \mathrm{BO}_{3}, 200 \mathrm{mg}$ $\mathrm{CoCl} 2.6 \mathrm{H} 2 \mathrm{O}, 900 \mathrm{mg} \mathrm{Na} 2 \mathrm{MoO}_{4} .2 \mathrm{H} 2 \mathrm{O}, 20 \mathrm{mg} \mathrm{NiCl} 2.6 \mathrm{H} 2 \mathrm{O}, 20 \mathrm{mg} \mathrm{Na} \mathrm{SeO}_{3}, 1000 \mathrm{~mL}$ distilled water (Meyer and Schlegel , 1983). The $\mathrm{pH}$ was adjusted to $\mathrm{pH} 5.5$ or $\mathrm{pH} 7.2 \mathrm{using}$ $\mathrm{HCl} 8 \mathrm{M}$ and $\mathrm{NaOH} 1 \mathrm{M}$ respectively.

The medium FM contained: $5 \mathrm{~g}$ yeast extract, $10 \mathrm{~g}$ nutrient broth, $1 \mathrm{~g}$ glucose, $1.75 \mathrm{~g}$ $\mathrm{K}_{2} \mathrm{HPO}_{4}, 0.75 \mathrm{~g} \mathrm{KH}_{2} \mathrm{PO}_{4}, 1000 \mathrm{~mL}$ distilled water (Völkl et al., 2004). The $\mathrm{pH}$ was adjusted to 5.5 and 7.2 using $\mathrm{HCl} 8 \mathrm{M}$ and $\mathrm{NaOH} 1 \mathrm{M}$ respectively and the medium was solidified with $20 \mathrm{~g}$ agar/L. 
Table 2: Pipetting scheme on microtiter plate for the microorganisms screening

\begin{tabular}{|l|l|l|l|l|l|l|l|l|l|l|l|l|}
\hline & $\mathbf{1}$ & $\mathbf{2}$ & $\mathbf{3}$ & $\mathbf{4}$ & $\mathbf{5}$ & $\mathbf{6}$ & $\mathbf{7}$ & $\mathbf{8}$ & $\mathbf{9}$ & $\mathbf{1 0}$ & $\mathbf{1 1}$ & $\mathbf{1 2}$ \\
\hline A & C & C & C & S1 & S1 & S1 & S2 & S2 & S2 & S3 & S3 & S3 \\
\hline B & S4 & S4 & S4 & S5 & S5 & S5 & S6 & S6 & S6 & S7 & S7 & S7 \\
\hline C & S8 & S8 & S8 & S9 & S9 & S9 & S10 & S10 & S10 & S11 & S11 & S11 \\
\hline D & S12 & S12 & S12 & S13 & S13 & S13 & S14 & S14 & S14 & S15 & S15 & S15 \\
\hline E & S16 & S16 & S16 & S17 & S17 & S17 & S18 & S18 & S18 & S19 & S19 & S19 \\
\hline F & S20 & S20 & S20 & S21 & S21 & S21 & S22 & S22 & S22 & S23 & S23 & S23 \\
\hline G & S24 & S24 & S24 & S25 & S25 & S25 & S26 & S26 & S26 & S27 & S27 & S27 \\
\hline H & S28 & S28 & S28 & S29 & S29 & S29 & S30 & S30 & S30 & S31 & S31 & S31 \\
\hline
\end{tabular}

C: Control

S: Microorganisms source

\subsubsection{Single culture selection of microorganisms}

A second screening of the bacterial and fungal pure culture that are able to grow on media containing enniatin as unique carbon source was made by incubating single cultures obtained from the initial screening. The bacteria were grown on liquid FM for 24 hours after which the wells of a microtiter plate were filled with $100 \mu \mathrm{L}$ of $\mathrm{MM}$ containing enniatins at the concentration $1 \mathrm{mg} / \mathrm{mL}(\mathrm{v} / \mathrm{v})$ and $10 \mu \mathrm{L}$ of bacteria suspension $(\mathrm{OD} 600=0.6)$ were added to the corresponding wells. Fungi were grown on FM agar plate for 5 days at $25^{\circ} \mathrm{C}$, a $5 \mathrm{~cm}$ agar plug was mixed in $2 \mathrm{~mL}$ sterile tap water and diluted 1/100 to get a spore suspension, $10 \mu \mathrm{L}$ of spores suspension were added to wells containing $100 \mu \mathrm{L} \mathrm{MM} / \mathrm{enniatins}(1 \mathrm{mg} / \mathrm{mL})$. The plates were incubated at $20^{\circ} \mathrm{C}$ for 3 and 5 days for bacteria and fungi respectively. The microorganisms obtained from the first selection with mixed cultures were inoculated on the $\mathrm{MM} / \mathrm{enniatins}$ having the same $\mathrm{pH}$ as the minimal media used for the first selection. After incubation, the content of each well was streaked on FM agar plates and the plates were incubated at $25{ }^{\circ} \mathrm{C}$. The number of microorganisms that remained alive was recorded. The control wells contained $100 \mu \mathrm{L}$ of $\mathrm{MM} /$ enniatins $(1 \mathrm{mg} / \mathrm{mL})$ and $10 \mu \mathrm{L}$ of sterile tap water. Three replicates were made for each microorganism and each medium. 


\subsection{Enniatin degradation}

\subsubsection{Enniatin degradation in the microtiter plates}

In order to check which of the microorganisms among those previously isolated was able to degrade enniatins, a minimal medium containing a limited amount of glucose and protein (MMGP) was used in addition to the previously used minimal medium. The composition of MMGP is as follows: MM with 5\% terrific broth (TB) (v/v) and $50 \mathrm{mg}$ glucose per liter medium (Völkl et al., 2004), the $\mathrm{pH}$ was adjusted to 7.2 using $\mathrm{NaOH} 1 \mathrm{M}$. TB consisted of $12.0 \mathrm{~g}$ tryptone, $24.0 \mathrm{~g}$ yeast extract and $2 \mathrm{ml}$ glycerin (Tartof and Hobbs, 1987). MMGP and $\mathrm{MM}(\mathrm{pH} 5.5$ or $\mathrm{pH}$ 7.2) containing enniatins were prepared to a final enniatin concentration of $1 \mathrm{mg} / \mathrm{mL}$.

Most of the microorganisms isolated were growing faster at $28^{\circ} \mathrm{C}$ than at $20^{\circ} \mathrm{C}$ on $\mathrm{FM}$ agar plates. Bacteria and fungi were pretreated as follows: bacteria were streaked on FM agar plates and incubated at $25^{\circ} \mathrm{C}$ for 24 hours. A single colony was mixed with sterile tap water, then the optical density (OD600) was measured and adjusted to $0.6,10 \mu \mathrm{L}$ of each bacteria suspension was added to a well of a 96-microtiter plate containing $100 \mathrm{~mL}$ of MM/enniatin or MMGP/enniatins. Spores of fungi previously isolated were counted using a Thoma chamber $\left(0.1 \mathrm{~mm}\right.$ depth, $0.0025 \mathrm{~mm}^{2}$ square area) and the suspension was diluted in order to have a final spore concentration of 10000 spores $/ \mathrm{mL}$. A $10 \mu \mathrm{L}$ of spores' suspension (100 spores) was mixed with $100 \mathrm{~mL}$ MM/enniatins or MMGP/enniatins in microtiter plates. The cultures were incubated at $28^{\circ} \mathrm{C}$, but no significant growth of microorganisms was observed in the wells after two days. So the experiment was suspended and repeated using $10 \mu \mathrm{L}$ bacterial suspension $(\mathrm{OD} 600=1) / 10 \mu \mathrm{L}$ fungal spores suspension (200 spores) mixed with $100 \mu \mathrm{L}$ MM/enniatins or MMGP/enniatins $\left(1 \mathrm{mg} / \mathrm{mL}\right.$ ) followed by incubation at $25^{\circ} \mathrm{C}$ during 3 and 5 days for bacteria and fungi respectively. The control wells contained $100 \mu \mathrm{L}$ of MM/enniatins $(1 \mathrm{mg} / \mathrm{mL})$ and $10 \mu \mathrm{L}$ of sterile tap water. Three replicates were made for each microorganism and each medium.

\subsubsection{Bacterial and Fungal enniatin degradation under shaking conditions}

Since bacteria and fungi grow faster in liquid media when shaken, this step was carried out in order to see if the degradation process improves. $250 \mu \mathrm{L}$ Bacteria suspension $(\mathrm{OD} 600=1)$ or $250 \mu \mathrm{L}$ spore suspension (50000 spores) were mixed with $2500 \mu \mathrm{L}$ MM/enniatins or 
MMGP/enniatins (final enniatin concentration: $1 \mathrm{mg} / \mathrm{mL}$ ), the cultures were shaken and incubated at $25^{\circ} \mathrm{C}$ during 3 and 5 days for bacteria and fungi respectively. Three replicates were made for each microorganism and each medium. The negative control was made by mixing $250 \mu \mathrm{L}$ of sterile tap water with $2500 \mu \mathrm{L}$ minimal medium containing enniatins at the final concentration $1 \mathrm{mg} / \mathrm{mL}$.

\subsection{Enniatin extraction and HPLC-UV analysis}

\subsubsection{Enniatin extraction}

The liquid culture of each microtiter plate well was collected and transferred into a $1.5 \mathrm{~mL}$ Eppendorf tube for the extraction of enniatins; the wells were rinsed using $100 \mu \mathrm{L} \mathrm{MeOH}$. Methanol was evaporated in the SpeedVac (Christ RVC 2-25 CD plus, $35^{\circ} \mathrm{C}, 30$ mbar). The content of each Eppendorf tube was diluted in $200 \mu \mathrm{L}$ ethyl acetate and $200 \mu \mathrm{L}$ water were added, each mixture was vortexed during $1 \mathrm{~min}$ and left for 2 hours at room temperature, the upper organic phase was carefully collected using a pipette and put into a new tube. Enniatin extraction was repeated twice using the same ethyl acetate volume, the organic phases were mixed and the solvent was evaporated in the SpeedVac as above. The dried samples containing enniatins were resuspended in $200 \mu \mathrm{L}$ methanol HPLC grade then transferred into HPLC vessels which were frozen at $-20^{\circ} \mathrm{C}$ until enniatin analysis by HPLC-DAD.

The cultures from the test-tubes (as mentioned above) were centrifuged at $4{ }^{\circ} \mathrm{C}$ ( $5 \mathrm{~min}, 4000$ RPM), $600 \mu \mathrm{L}$ of the supernatant was collected and transferred to a new tube, enniatins and degradation products were extracted twice using $600 \mu \mathrm{L}$ EtOAc. For extraction, the tubes were vortexed during $2 \mathrm{~min}$ and left at room temperature for $30 \mathrm{~min}$. The upper ethyl acetate phase was carefully collected using a pipette and put in a new tube. The extraction was done twice, then the organic phases were mixed together and the solvent was evaporated in the SpeedVac as above. The dried samples were resuspended in $600 \mu \mathrm{L}$ methanol HPLC grade then transferred into HPLC vessels which were frozen at $-20{ }^{\circ} \mathrm{C}$ until enniatin analysis by HPLCUV. 


\subsubsection{HPLC-UV analysis}

The method used to analyze the ethyl acetate extract from the cultures of microorganisms is identical to the method developed and described under the subtitle "2.1.2. Extraction of enniatins and High Performance Liquid Chromatography analysis" of the previous chapter of this thesis. After HPLC-UV analysis, the percentage of reduction of enniatins by each microorganism for three repetitions was calculated.

\subsection{Identification of degradation products}

Analysis of enniatin degradation products was achieved using the following procedure: first a characterization of the compound isolated using HPLC-MS with the modality full scan was done using $\mathrm{m} / \mathrm{z}$ range from 50 to $800 \mathrm{Da}$ to obtain general spectra of the degradation compounds. Then the results were processed using the script Perl in order to generate a heatmap of the fragments, and new signals present in the treated samples were sorted out. Finally the mass spectra of the potential product were checked and their possible molecular formula was deduced using ChemCalc (2013).

\subsubsection{HPLC-MS analysis}

The separation was carried out using a mass spectrometer system made of solvent delivery module (Varian Prostar 210), an autosampler (Varian Prostar 410), an ion trap 800 MS, an oven (Varian Model 510 Prostar). A C18 reversed phase column (Polaris C18-Ether, HPLC column, $180 \AA, 5 \mu \mathrm{m}, 100 \times 2.0 \mathrm{~mm}$, Agilent) in an oven (temperature set at $40^{\circ} \mathrm{C}$ ) was used. Liquid chromatography conditions were set up using a constant flow rate at $0.2 \mathrm{ml} / \mathrm{min}$. Methanol and water $(7 \mathrm{mM}$ acetic acid $+0.01 \mathrm{mM}$ sodium acetate) were used as mobile phase in gradient elution consisting of a linear increase of methanol from $2 \%$ to $98 \%$ for 40 minutes, methanol was then maintained at $98 \%$ for 5 minutes, decreased to $2 \%$ for 2 minutes and finally held for 8 minutes at $2 \%$. The injection volume was $10 \mu \mathrm{L}$. The instrument was configured in the positive ionization mode. The protonated ions 640.4; 654.4; 668.8; and 682.4 were used as precursor ions for enniatins B, B1, A1 and A respectively. The sodium adduct ions $662.4 ; 676.4 ; 690.8 ; 704.4$ were used as precursor ions for enniatins B, B1, A1 and A respectively. 


\section{Results}

\subsection{Selection of microorganisms from mixed cultures- Binocular microscopic observation of the microtiter plates}

The pictures below show some wells of the microtiter plates containing microorganisms from various sources inoculated in a minimal media (MM) containing enniatin as sole carbon source $(1 \mathrm{mg} / \mathrm{mL})$. The wells present differences relative to color and consistency depending on the origin of the microorganism. The control well remained colorless and translucent, while some other wells showed a colloidal aspect or a change in color varying from creamy, reddish, greenish or blackish. Furthermore, green/grey or white mycelium could be observed in some wells. After incubation of each microorganism in the medium containing enniatin, dilution 1/10 and re-incubation for 21 days, some wells were observed under a binocular microscope. The wells of the control (containing medium and enniatins) (Figure 2-A, B and C) showed aggregated enniatin crystals mostly planning inside liquid medium or at its surface. The wells of some samples under the binocular microscope are shown on Figure 3. In contrary to control wells, a change in texture and turbidity of cultures in some wells was observed containing sample supernatants. Mycelium was present in some wells (example: Figure 3, B, C, D), while in others the turbidity of medium increased or the combination of both changes was observed (example: Figure 3, E, F, D). Also the enniatin crystals in wells containing sample supernatants were dislocated into smaller pieces and scattered all around the medium (example: Figure 3, A, D, E, F). However, the observations on the binocular microscope showed some wells presenting a colloidal solution or a disappearance of enniatin crystals, but no microorganisms were isolated from those wells, some microorganisms were able to grow in the minimal medium containing enniatin as unique carbon source but did not grow on the agar plates used, thus they could not be isolated during these experiments. 

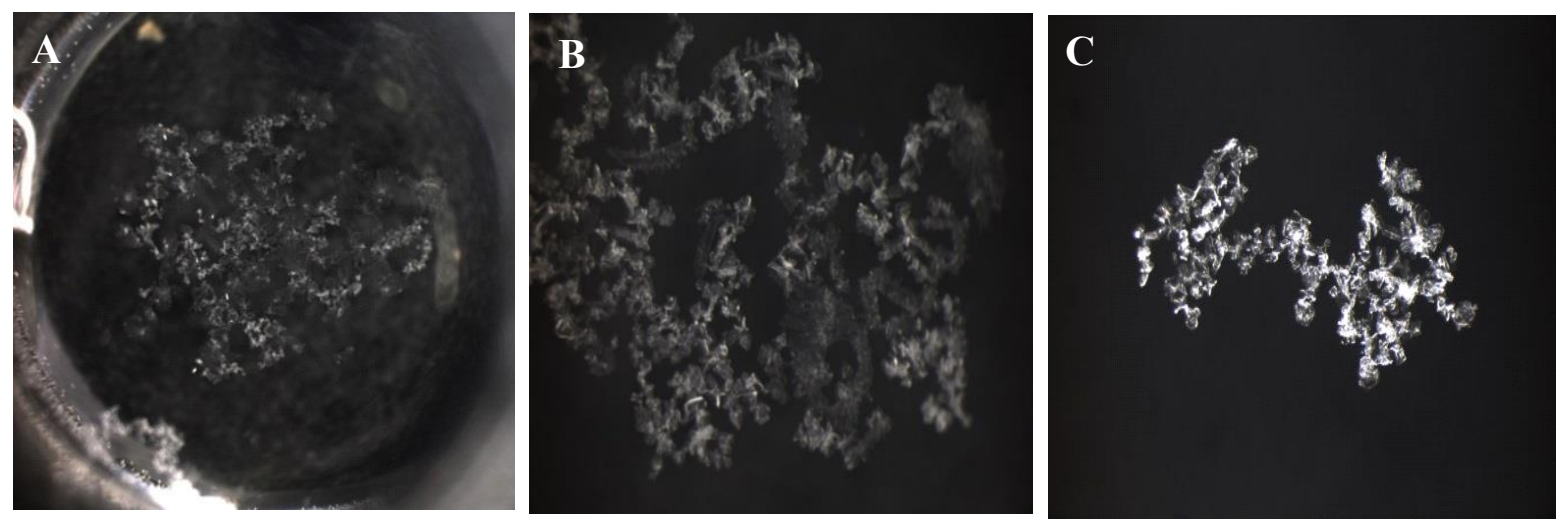

Figure 2: Microtiter plate wells of control under binocular microscope after 1/10 dilution of the cultures and 20 incubation days at $20^{\circ} \mathrm{C}$. A: resolution 20x, B and C: resolution 80x.
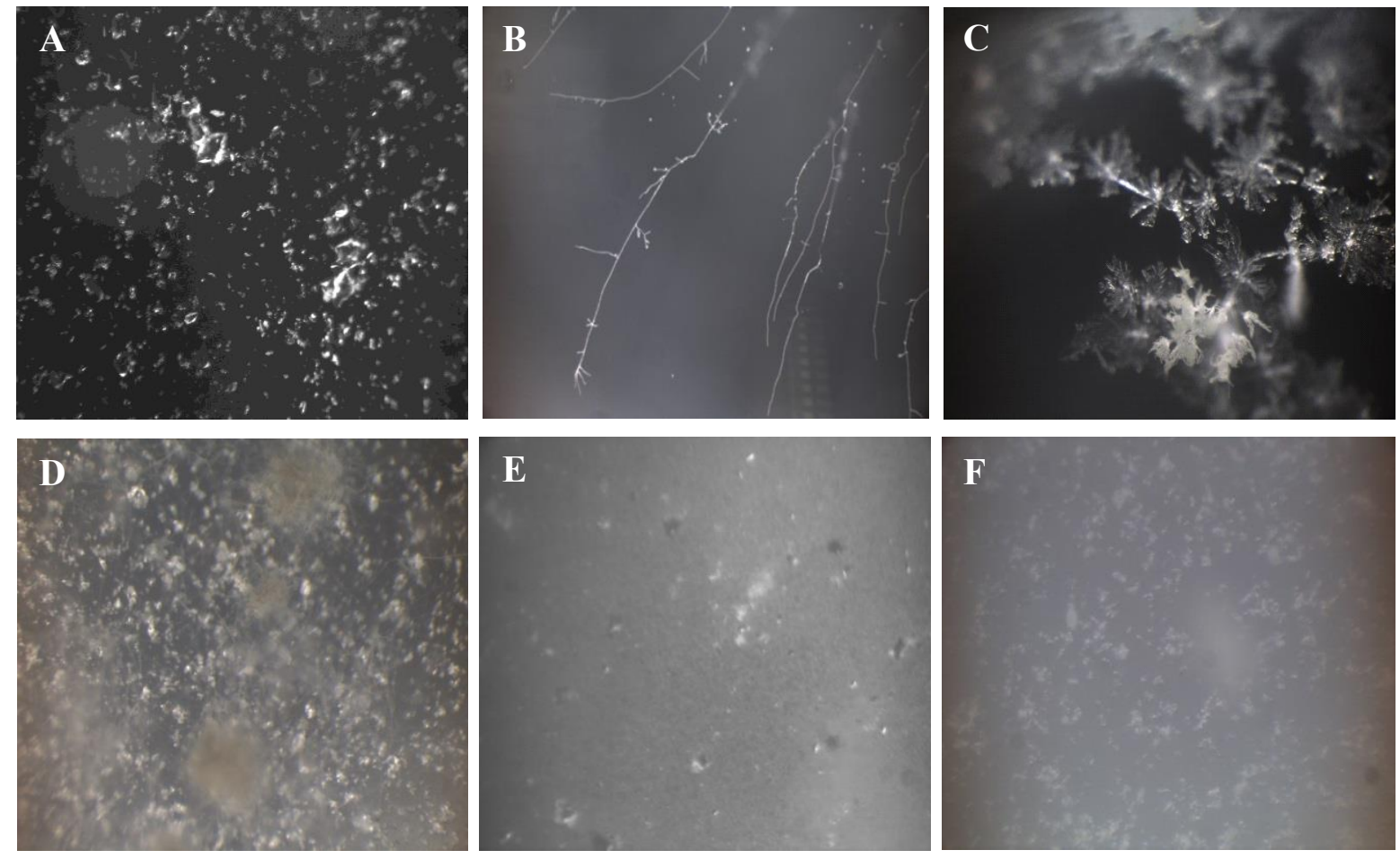

Figure 3: Microtiter plate wells of some samples under the binocular microscope (resolution 80x) after $1 / 10$ dilution of the cultures and 21 incubation days at $20{ }^{\circ} \mathrm{C}$. A \& B: maize sample; C: almond sample, D: peanut sample, E: Water Lake Kiessee sample and F: amaranth sample. 


\subsection{Microorganisms isolated from mixed cultures}

Microorganisms isolated on the basis of their ability to grow on MM containing enniatins as unique carbon source are summed up in Table 3.

Table 3: Number and kind of microorganisms isolated from each source used.

\begin{tabular}{|c|c|c|c|c|c|}
\hline & \multirow[t]{2}{*}{ Microorganism sources (S) } & \multicolumn{2}{|c|}{$\begin{array}{c}\text { Bacteria and Yeast- } \\
\text { like }\end{array}$} & \multicolumn{2}{|c|}{ Fungi } \\
\hline & & PH 7.2 & PH 5.5 & PH 7.2 & PH 5.5 \\
\hline \multirow[t]{10}{*}{ Grains } & S1: Amaranth & 3 & 1 & 0 & 0 \\
\hline & S2: Summer wheat & 0 & 0 & 0 & 2 \\
\hline & S3: Maize & 5 & 4 & 0 & 2 \\
\hline & S4: Yellow soybean & 0 & 0 & 0 & 0 \\
\hline & S5: Balium elarive & 0 & 0 & 1 & 0 \\
\hline & S6: Mini maize & 0 & 0 & 1 & 1 \\
\hline & S7: Sorghum & 2 & 3 & 0 & 0 \\
\hline & S8: Rice & 2 & 0 & 0 & 0 \\
\hline & S9: White beans & 0 & 0 & 0 & 0 \\
\hline & S10: Summer rape & 0 & 0 & 1 & 1 \\
\hline \multirow[t]{4}{*}{ Nuts } & S11: Hazelnut & 2 & 0 & 0 & 0 \\
\hline & S12: Almond & 0 & 0 & 2 & 3 \\
\hline & S13: Mung bean & 2 & 0 & 0 & 1 \\
\hline & S14: Peanuts & 3 & 0 & 2 & 1 \\
\hline \multirow[t]{3}{*}{ Water } & S15: Lake Wendebachstausee & 4 & 8 & 0 & 0 \\
\hline & S16: River Leine Kanal & 6 & 6 & 0 & 0 \\
\hline & S17: Lake Kiessee & 5 & 6 & 0 & 0 \\
\hline \multirow[t]{4}{*}{ Soil } & $\begin{array}{l}\text { S18:Wheat field soil } \quad \text { (around } \\
\text { Wendebachstausee) }\end{array}$ & 5 & 2 & 1 & 0 \\
\hline & S19: Garden soil & 8 & 4 & 0 & 0 \\
\hline & S20: Wheat field soil (Weende Nord) & 6 & 0 & 0 & 0 \\
\hline & S21: Maize field soil & 11 & 2 & 1 & 0 \\
\hline \multirow[t]{3}{*}{ Juices } & S22: Apple juice & 6 & 6 & 0 & 0 \\
\hline & S23: Orange juice & 0 & & 0 & 0 \\
\hline & S24: Tomato juice & 0 & 2 & 0 & 0 \\
\hline \multirow[t]{4}{*}{ Noodle } & S25: Chinese noodle & 0 & 0 & 0 & 0 \\
\hline & S26: Noodle Penne & 0 & 0 & 0 & 1 \\
\hline & S27: Vegetarian Noodle Vietnam & 0 & 0 & 0 & 0 \\
\hline & $\begin{array}{l}\text { S28: Noodle Echt Schwäbische } \\
\text { Frischei-Nudeln }\end{array}$ & 0 & 0 & 0 & 0 \\
\hline Strains & S29: 5 fungal strains from forest & & & & \\
\hline \multirow[t]{2}{*}{ Fruits } & S30: Plums & 0 & 0 & 0 & 1 \\
\hline & S31: Raisin & 0 & 0 & 0 & 0 \\
\hline Total & & 70 & 44 & 10 & 24 \\
\hline
\end{tabular}




\section{Bacteria and Yeast-like microorganisms}

In total 114 bacteria and yeast-like microorganisms were isolated from different sources after incubation in minimal medium (MM) containing enniatin as sole carbon source at $\mathrm{pH}$ 7.2. Bacteria and yeast-like microorganisms were differentiated depending on their colony characteristics on full medium (FM) agar plates. The major sources of bacteria and yeast-like microorganisms were: soil from maize field, followed by garden soil from which respectively 11 and 8 sorts of bacteria and yeast-like microorganisms were isolated. In general, most bacteria and yeast-like microorganisms were isolated from soil samples (38) and water samples (35). The number of bacteria and yeast- like microorganisms isolated from the MM with $\mathrm{pH} 7.2$ (70 bacteria) was higher than those isolated from MM with $\mathrm{pH} 5.5$ (44). No bacteria and yeast-like microorganisms were isolated from noodle and plums and raisin samples.

\section{Fungi}

In total, 34 fungi were isolated on their ability to grow on MM containing enniatin as sole carbon source. The distinction among fungi was made essentially based on colony visual characteristics on FM agar plates. The main sources were nuts (hazelnuts, almonds and peanuts) and grains (summer wheat and maize) from which 15 and 5 fungi were isolated respectively. A majority of fungi were isolated from $\mathrm{MM}$ with $\mathrm{pH}$ value 5.5.

\subsection{Single culture selection of microorganisms}

Out of the 148 microorganisms previously isolated 20 bacteria and 10 fungi could not be cultivated. When the pure cultures previously isolated were incubated as single cultures in MM/enniatin (enniatin final concentration: $1 \mathrm{mg} / \mathrm{mL}$ ), 6 bacteria and 1 fungus did not grow on the MM medium containing enniatin as sole carbon source. The sources of these bacteria were garden soil (2 bacteria), wheat field from Weende Nord in Göttingen (1 bacterium), apple juice (1 bacterium) and the tomato juice (1 bacterium) and wheat field around Wendebachstausee in Göttingen (1 bacterium). The fungus which didn't grow on MM/enniatin as single culture was isolated from the noodle sample. This reduced the number of microorganisms isolated to 88 and 23 for bacteria and fungi respectively. 


\subsection{HPLC-UV analysis of culture media}

Enniatin concentrations were generally reduced in all samples compared to the control (data not shown). The reduction was variable depending on the enniatin variant and the microorganism used. The reduction rate was comparable in both media used (MM and MMGP) for bacteria, whereas with fungi there seemed to be a greater enniatin reduction, when incubated in MMGP compared to MM. The concentration of enniatin A was reduced most. With the exception of one bacterium (K4) and two fungi (F18 and F20) the pattern of the chromatograms peaks and the UV spectra were similar to that of the control sample.

For bacterium K4, the retention times of the peaks observed after HPLC-UV analysis of the cultures are summarized in Table 4. In the control samples, the retention times of enniatins B, $\mathrm{B} 1$, $\mathrm{A} 1$ and $\mathrm{A}$ were $7.68 \mathrm{~min} ; 8.11 \mathrm{~min} ; 8.48 \mathrm{~min}$ and $8.8 \mathrm{~min}$ respectively. In the chromatograms of samples of the bacterium $\mathrm{K} 4$ a new peak was observed with retention time of $7.28 \mathrm{~min}$ which is present neither in the control nor in the chromatogram of the bacterium K4 incubated in the media without enniatin. HPLC-UV chromatograms of control and cultures of bacterium K4 incubated in MM or in MMGP are presented in Figure 4.

Table 4: Retention times of peaks observed on the HPLC-UV chromatograms resulting from the incubation of the control sample and the fungus K4 in MM and MMPG containing enniatins $(1 \mathrm{mg} / \mathrm{mL})$.

\begin{tabular}{lccc}
\hline Peaks & \multicolumn{3}{c}{ Retention times } \\
\cline { 2 - 4 } & Control & K4 in MM & K4 in MMGP \\
\hline New peak & & 7.28 & 7.28 \\
Enniatin B & 7.68 & 7.68 & 7.73 \\
Enniatin B1 & 8.11 & 8.11 & 8.13 \\
Enniatin A1 & 8.48 & 8.48 & 8.48 \\
Enniatin A & 8.80 & 8.85 & 8.85 \\
\hline
\end{tabular}



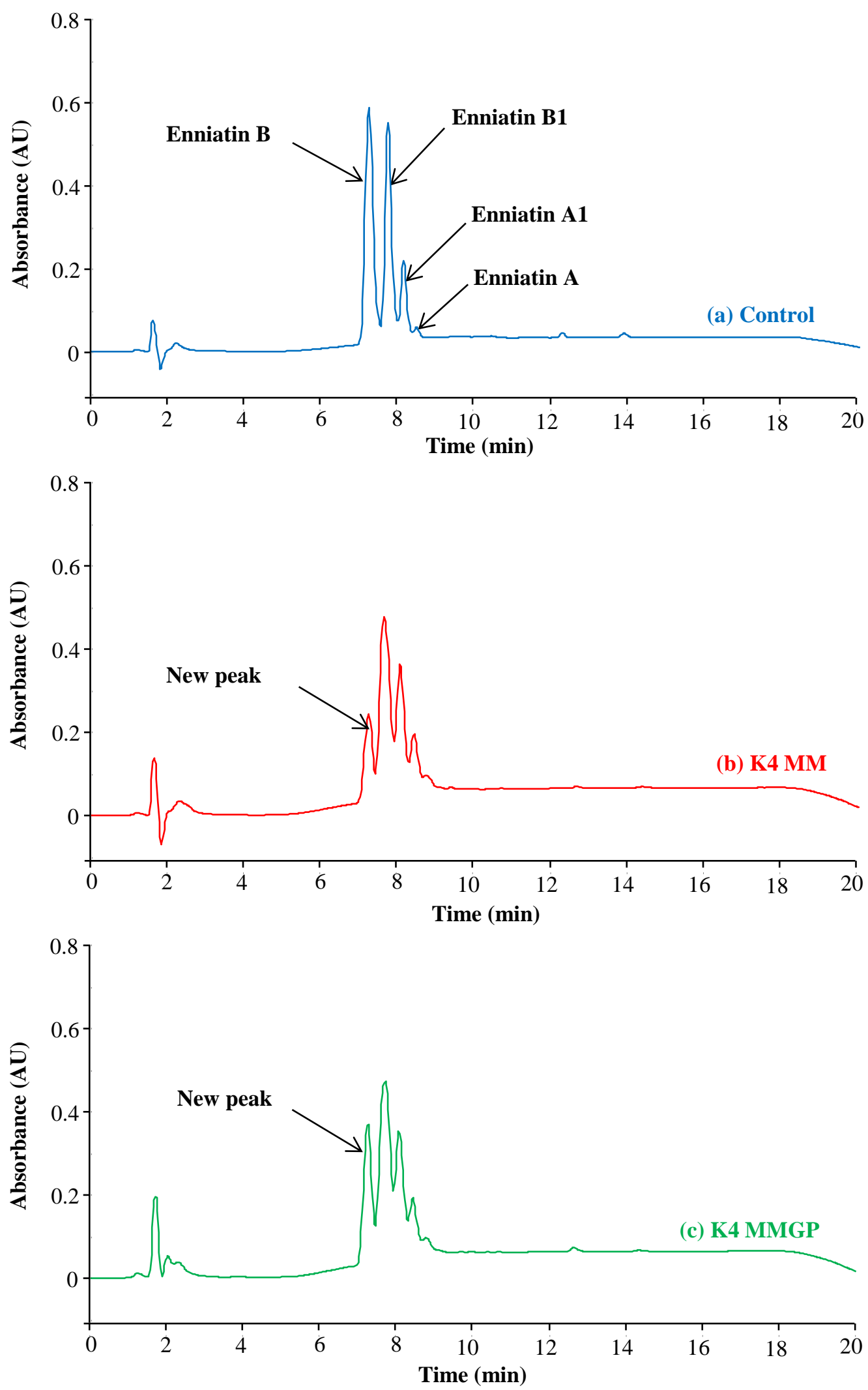

Figure 4: HPLC-UV chromatograms of enniatins and related degradation products present in the growth medium after incubation with bacteria $\mathrm{K} 4$ for 3 days at $25^{\circ} \mathrm{C}$. 
The chromatograms of samples containing the fungi F18 and F20 showed a different pattern when the fungus was incubated in the medium MM compared to the medium MMGP. Table 5 (1 and 2) summarizes the retention times of peaks observed on the chromatograms resulting from the incubation of fungi F18 and F20 in MM and MMPG containing enniatins and the control sample. New peaks eluting before the four enniatin variants were observed. In general it was not simple to match the peaks in the chromatograms of the treated samples and those in the control sample. HPLC-UV chromatograms of treated cultures of fungi F18 and F20 are shown in Figure 5 and Figure 6 respectively.

Table 5: Retention times of peaks observed on the HPLC-UV chromatograms resulting from the incubation of the control sample and the fungus F20 (Table 5.1) and fungus F18 (Table 5.2) in MM and MMPG containing enniatins ( $1 \mathrm{mg} / \mathrm{mL})$.

1.

\begin{tabular}{lccc}
\hline Peaks & \multicolumn{3}{c}{ Retention times } \\
\cline { 2 - 4 } & Control & F20 in MM & F20 in MMGP \\
\hline New peak & & 6.19 & 4.67 \\
& & 6.96 & 5.28 \\
Enniatin B & 7.68 & 7.57 & 6.56 \\
Enniatin B1 & 8.11 & 8.03 & 7.23 \\
Enniatin A1 & 8.48 & 8.40 & 8.03 \\
Enniatin A & 8.80 & 8.72 & 8.51 \\
\hline
\end{tabular}

2.

\begin{tabular}{lccc}
\hline Peaks & \multicolumn{3}{c}{ Retention times } \\
\cline { 2 - 4 } & Control & F18 in MM & F18 in MMGP \\
\hline New peak & & 3.47 \\
& & 3.87 \\
& & & 4.91 \\
& & & 5.68 \\
Enniatin B & 7.31 & 7.96 & 6.64 \\
Enniatin B1 & 7.79 & 7.81 & 7.28 \\
Enniatin A1 & 8.19 & 8.21 & 7.79 \\
Enniatin A & 8.53 & 8.56 & 8.19 \\
\hline
\end{tabular}



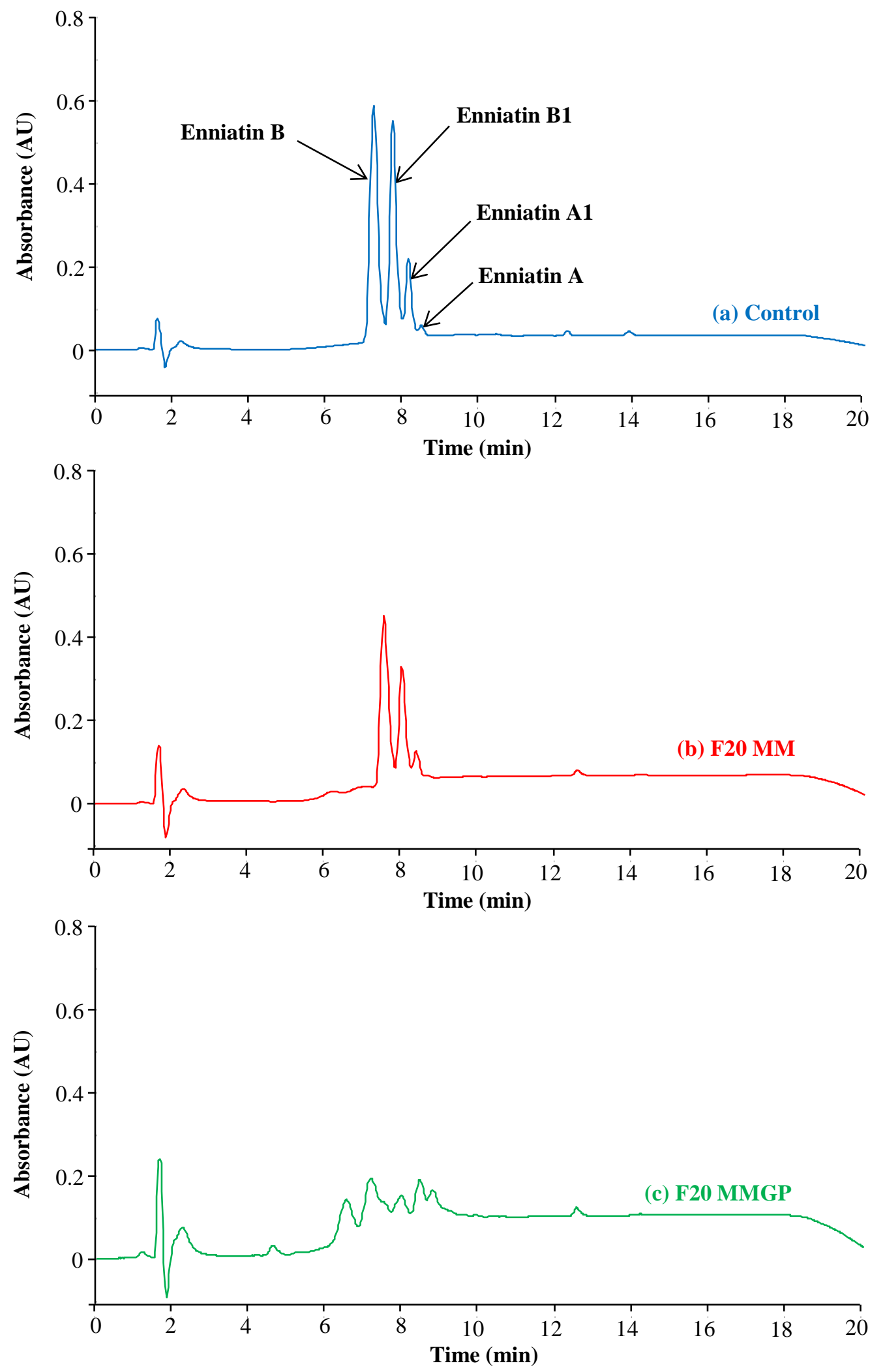

Figure 5: HPLC-UV chromatograms of enniatins and related degradation products present in the growth medium after incubation with fungi $\mathrm{F} 20$ for 5 days at $25^{\circ} \mathrm{C}$. 

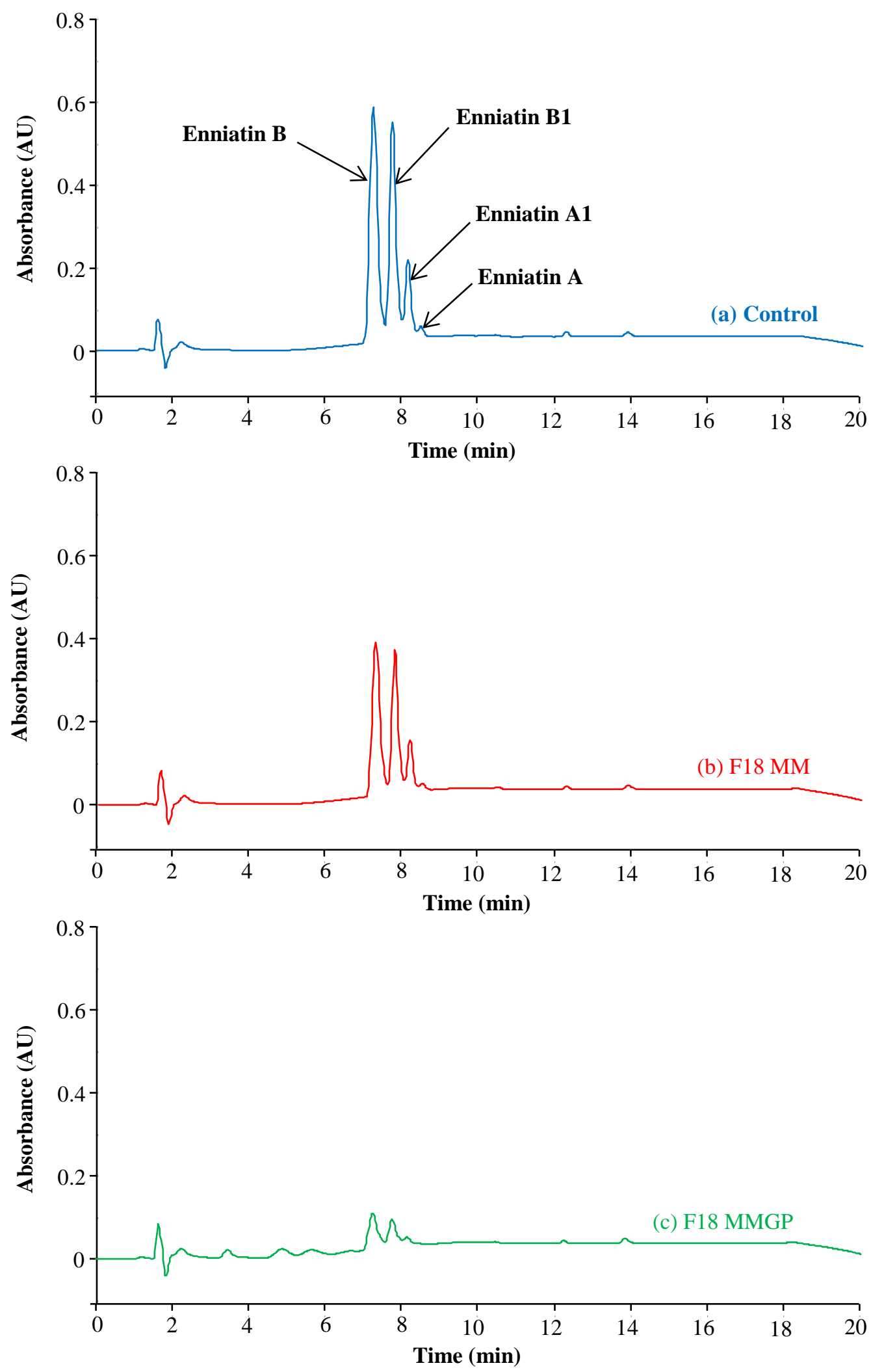

Figure 6: HPLC-UV chromatograms of enniatins and related degradation products present in the growth medium after incubation with fungi $\mathrm{F} 18$ for 5 days at $25^{\circ} \mathrm{C}$. 
In contrast to the HPLC-UV chromatogram of the bacterium K4, the chromatogram patterns of the fungi F20 and F18 were different when the fungi were cultured in MM or in MMGP. When the fungi F18 was incubated in MM containing enniatins, no new peak was observed, while in MMGP, new peaks were detected having lower retention times than those of enniatin variants (Table 5.2). Two and three new peaks (eluted before enniatin peaks) were detected on the HPLC-UV chromatogram of the fungi F20 after incubation in MM and MMGP respectively (Table 5.1). But in general, the matching of the peaks with enniatin peaks in the control was not conclusive.

\subsection{Enniatin degradation under shaking conditions}

When microorganisms isolated on the basis of their property to grow on media containing enniatins as unique carbon source were incubated on MM and on MMGP, growth (turbidity for bacteria/ mycelium for fungi) was generally observed in all the samples of bacteria and fungi. After visual observation, mycelium was more abundant in MMGP than in MM, while the turbidity of bacteria cultures was unaffected by the medium used as shown in Figure 7.

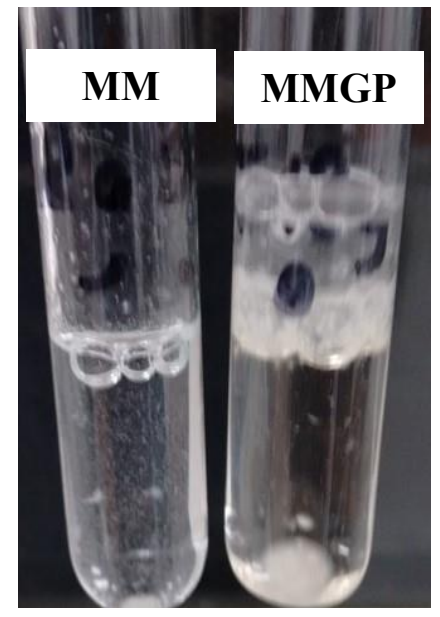

A

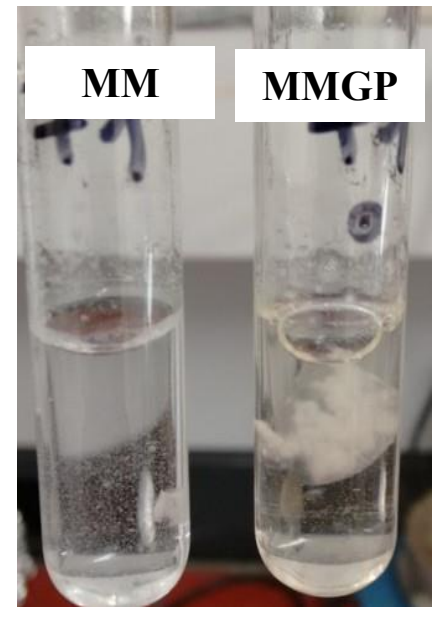

B

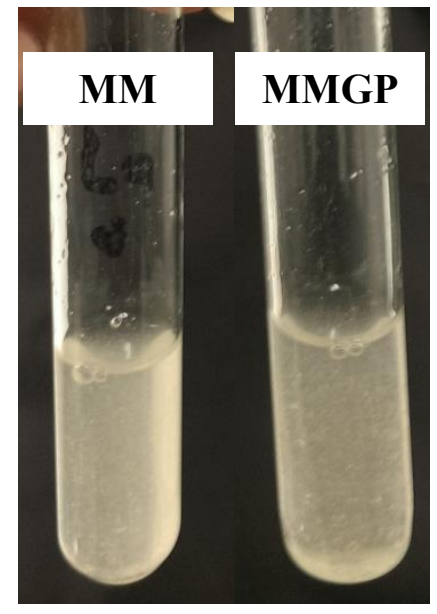

C

Figure 7: Cultures of two fungi (A and B) and one bacterium (C) in MM or in MMGP both containing enniatins after 5 days (fungi) and 3 days (bacterium) incubation at $25^{\circ} \mathrm{C}$.

The HPLC-UV analysis of the pure cultures of the bacterium and the fungi incubated with MM or MMGP while shaking did not show any difference regarding the chromatogram pattern. The chromatograms of bacterium K4 and fungi F18 and F20 were identical to those obtained when the microorganisms were incubated in MM and MMGP in the microtiter plate without shaking during the incubation. 


\subsection{Identification of degradation products using HPLC-MS}

The culture media of fungi F18, F20 and bacterium K4 incubated with enniatins in MMGP for 3 days (bacterium) or 5 days (fungi) were analyzed using LC-MS in full scan mode and a positive ionization mode using the $\mathrm{m} / \mathrm{z}$ range from 50-800 Da. The control was made of the medium MMGP containing enniatins. The total ion chromatograms (TIC) of culture supernatants are presented in Figures 8. The TIC of microorganisms grown in MMGP medium without enniatins is presented in Figure $\mathrm{i}$ in Appendix. Figure 8 shows the presence of enniatins B, B1, A1 and A in the control sample with the retention times 35.379 min; $36.338 \mathrm{~min} ; 37.308 \mathrm{~min} ; 38.113 \mathrm{~min}$ respectively.

ENN: enniatin 


\section{Control}

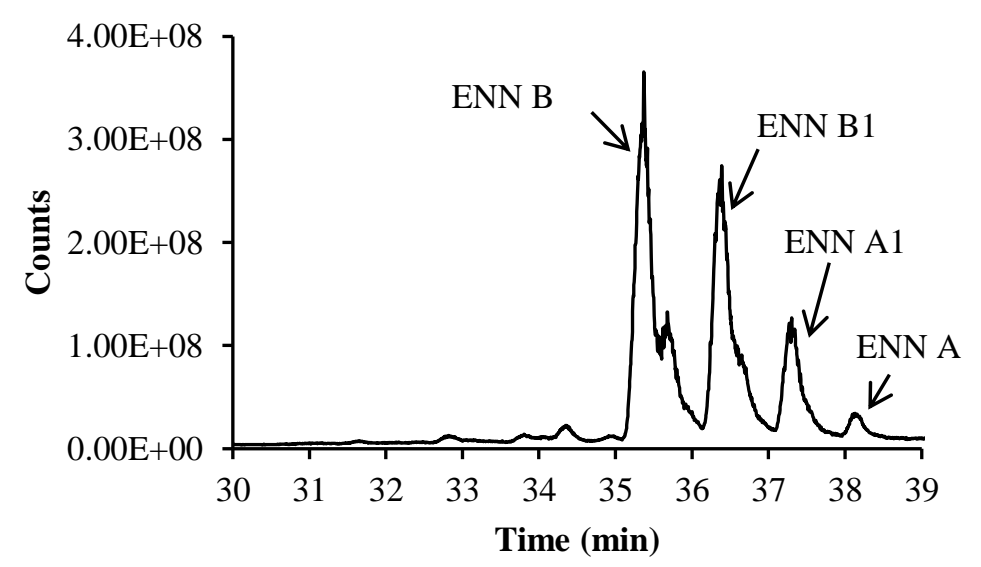

Fungus F18

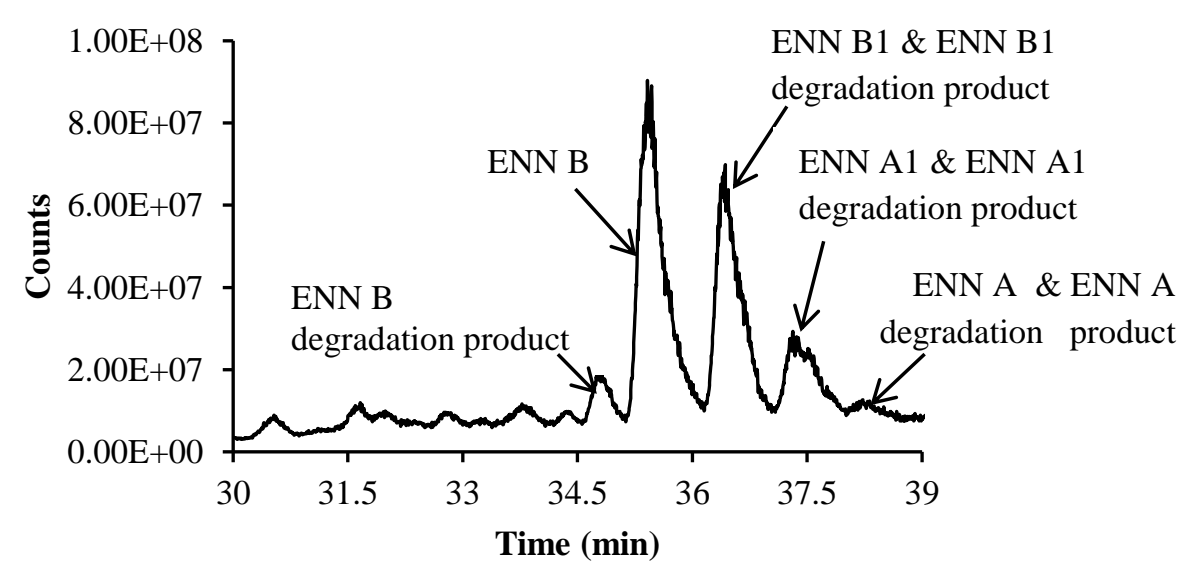

Bacterium K4 ENN A1 degradation

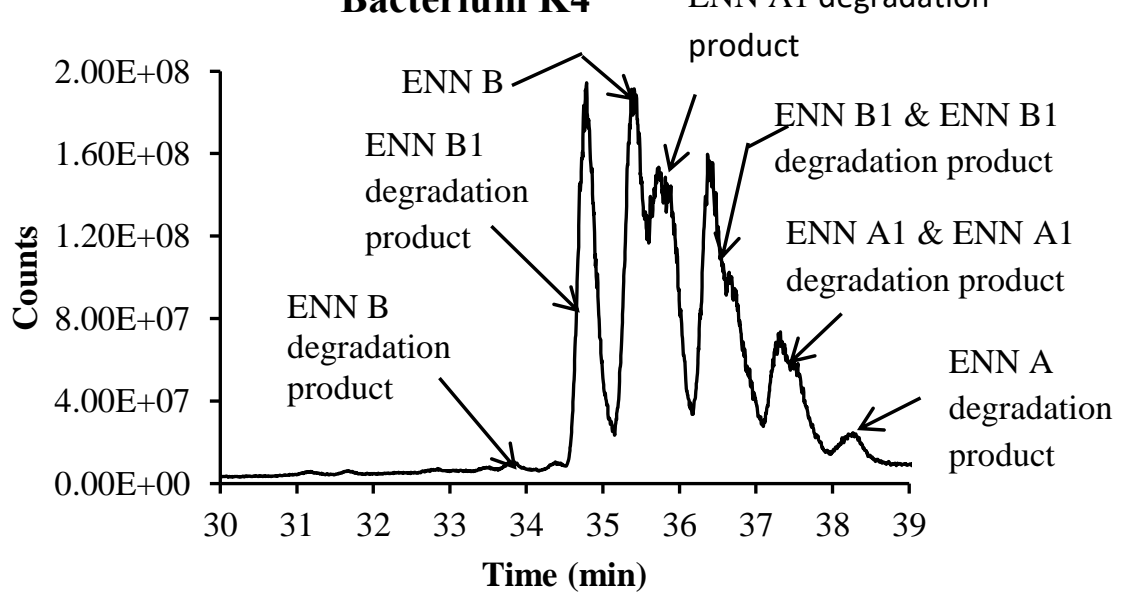

Fungus F20

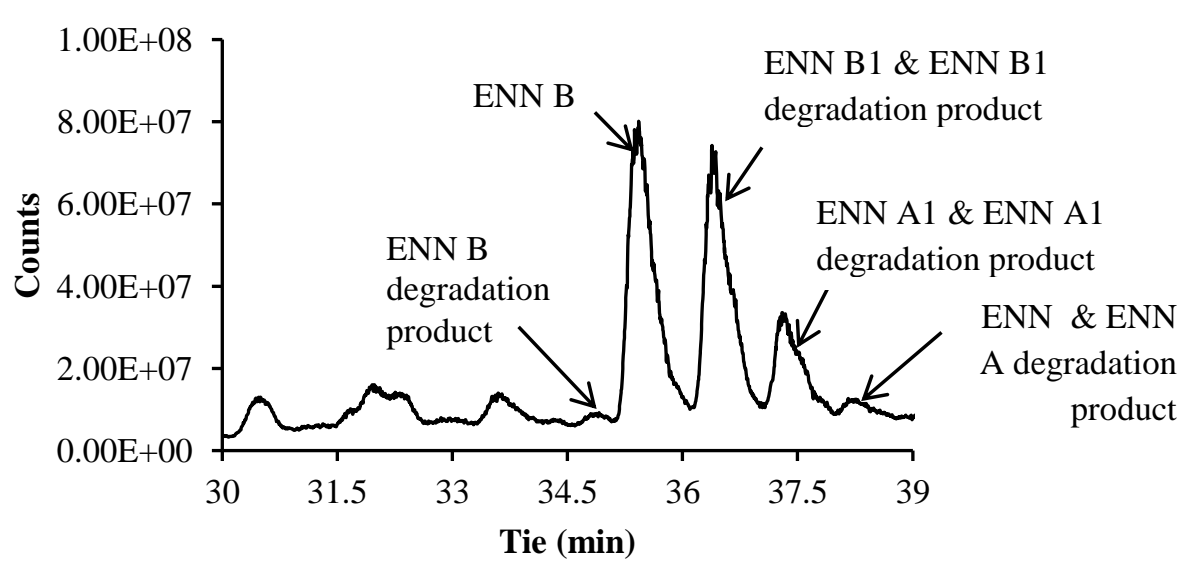

Figure 8: Total ion chromatograms of culture supernatants of control, bacterium K4, fungi F18 and F20 incubated in MMGP for 3 days (K4) and 5 days (fungi) at $25^{\circ} \mathrm{C}$. 


\subsubsection{Degradation product of enniatin B}

The Figures 9, 10 and 11 show the extracted ion chromatograms (EIC) of the degradation product of enniatin B in the culture supernatant of bacterium K4 and fungi F18 and F20 respectively. The mass spectra related to the enniatin B degradation compound identified with $[\mathrm{M}+\mathrm{H}]^{+}$and $[\mathrm{M}+\mathrm{Na}]^{+}$at $\mathrm{m} / \mathrm{z} 658.08$ and 680.4 respectively are also presented. In the EIC of the culture supernatant of bacterium K4 (Figure 9) the enniatin B degradation product appears as a double peak with retention times $33.5 \mathrm{~min}$ and $33.8 \mathrm{~min}$, while the residual enniatin $\mathrm{B}$ in culture is present as a peak with the retention time $35.4 \mathrm{~min}$. The product of enniatin $\mathrm{B}$ degradation in the culture supernatant of the fungi F18 and F20 appears as a double peak eluted at $33.3 \mathrm{~min}$ and $34.9 \mathrm{~min}$ respectively (Figure 10 and 11). In the EIC of enniatin B degradation product of the sample $\mathrm{K} 4$, both peaks representing enniatin B degradation product were dominated by the protonated ion compared to the sodium adduct ion, whereas the opposite was observed for both fungi F18 and F20. The molecular formula of the protonated ion of enniatin $\mathrm{B}$ degradation product is $\mathrm{C}_{33} \mathrm{H} 60 \mathrm{~N}_{3} \mathrm{O} 10$. 


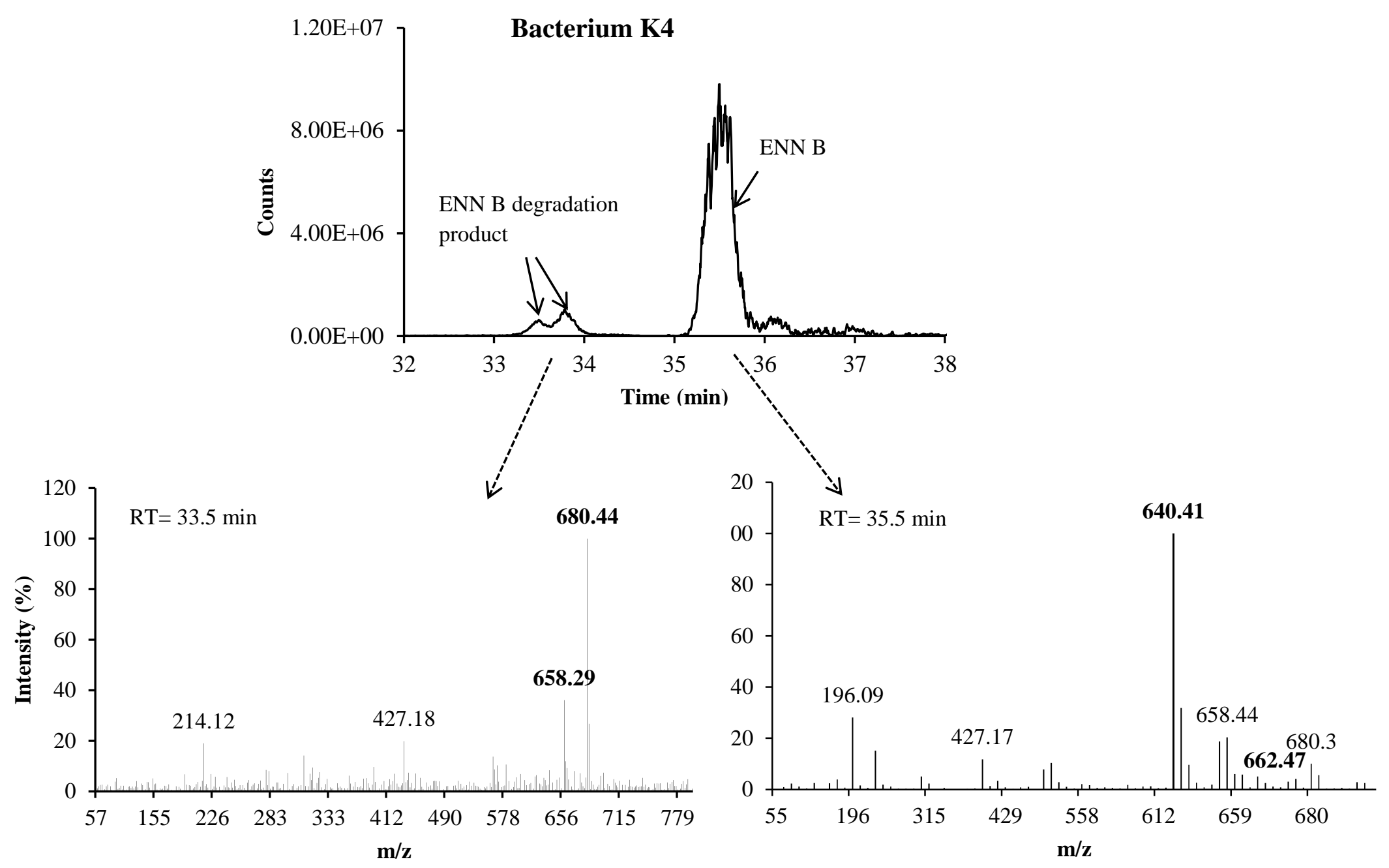

Figure 9: EIC and mass spectra of enniatin B degradation product by bacterium K4 with $[\mathrm{M}+\mathrm{H}]^{+}$658.29. 

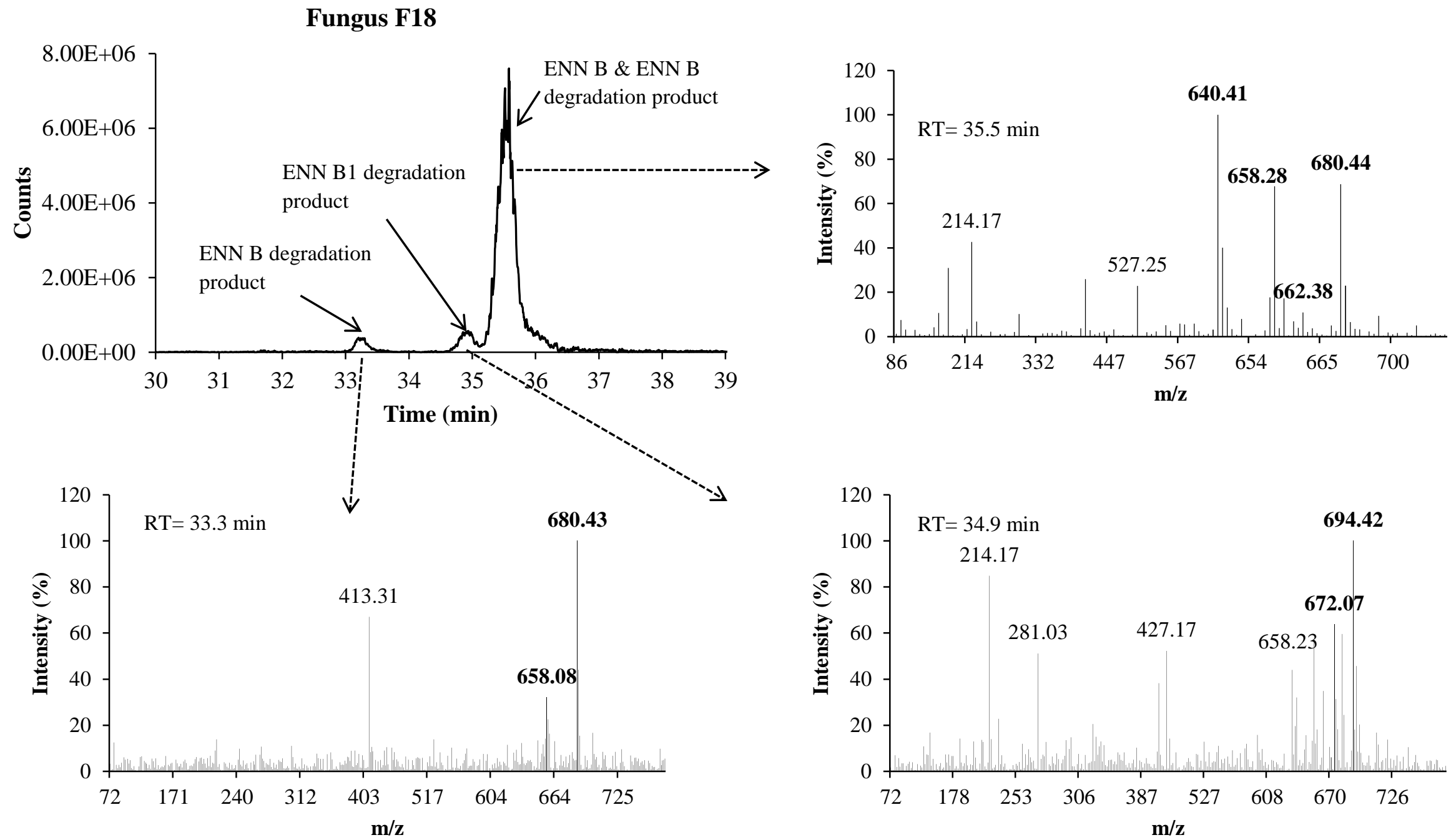

Figure 10: EIC and mass spectra of enniatin B degradation product by fungus F18 with $[\mathrm{M}+\mathrm{H}]^{+} 658.08$ or 658.28 . 


\section{Fungus F20}
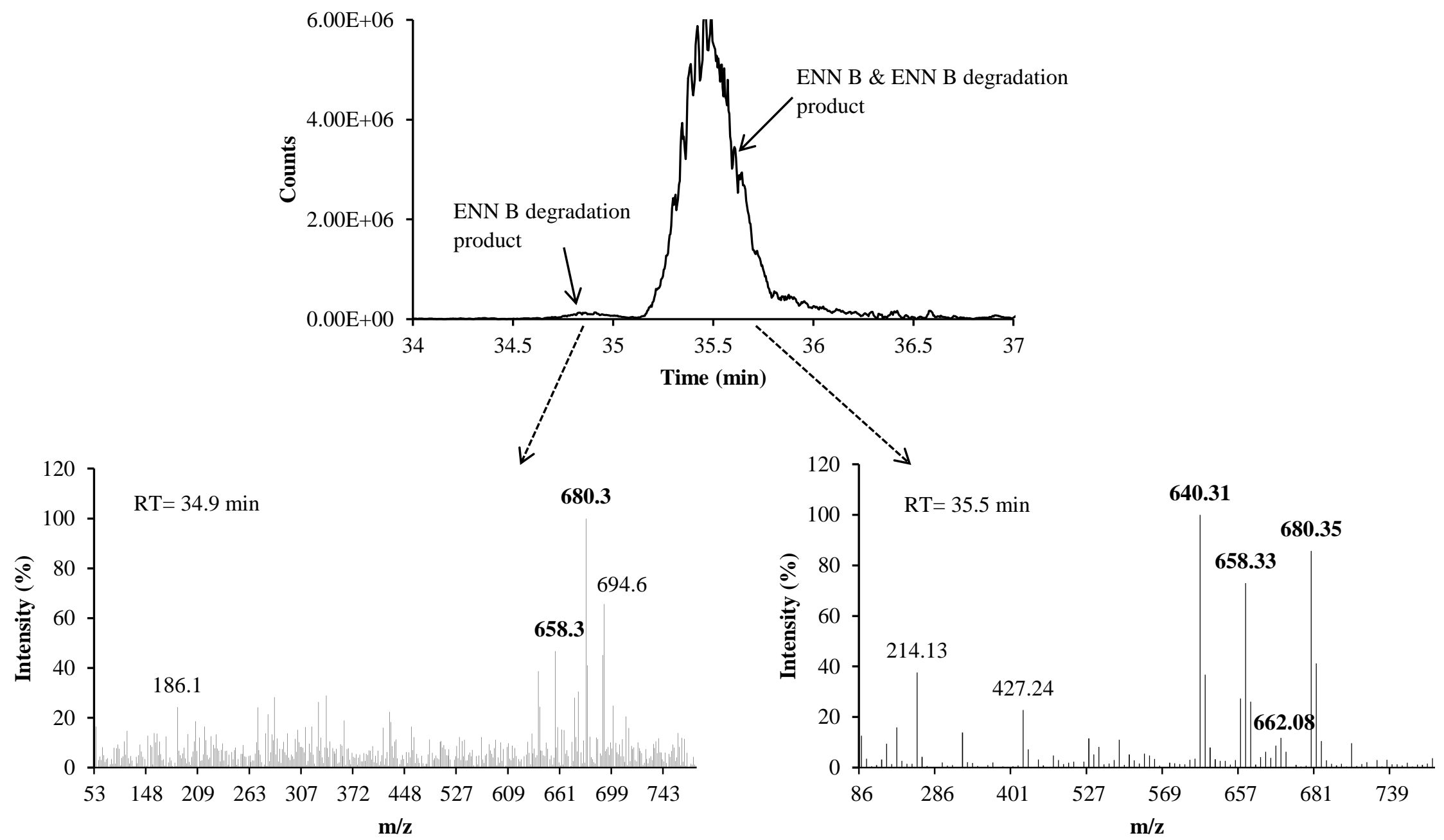

Figure 11: EIC and mass spectra of enniatin B degradation product by fungus $\mathrm{F} 20$ with $[\mathrm{M}+\mathrm{H}]^{+} 658.3$. 


\subsubsection{Degradation product of enniatin B1}

The EIC of the degradation product of enniatin B1 and the related mass spectra of the peaks observed are shown in Figures 12, 13 and 14. The enniatin B1 degradation product resulting from the incubation of bacterium K4 (Figure 12) and both fungi F18 and F20 (Figure 13 and 14 respectively) in MMGP containing a mixture of enniatins $\mathrm{A}, \mathrm{A} 1, \mathrm{~B} 1$ and $\mathrm{B}$ was demonstrated by the extracted ion chromatogram (EIC) of the compound with $\mathrm{m} / \mathrm{z} 672.3$ for the $[\mathrm{M}+\mathrm{H}]^{+}$and 694.4 for $[\mathrm{M}+\mathrm{Na}]^{+}$showing two peaks with identical mass spectrum. The retention time of enniatin $\mathrm{B} 1$ in the culture medium was $36.5 \mathrm{~min}$. The first peak of the enniatin $\mathrm{B}$ degradation product corresponding to the $\mathrm{m} / \mathrm{z} 672.3[\mathrm{M}+\mathrm{H}]^{+}$eluted before enniatin B1 with a retention time of 34.8 min indicating that the compound is more hydrophilic than the parent compound, while the second peak had a retention time of $36.5 \mathrm{~min}$ and overlapped with enniatin B1. In the EIC of enniatin B1 degradation product of the sample $\mathrm{K} 4$, both peaks representing enniatin B1 degradation product were dominated by the protonated ion compared to the sodium adduct ion, whereas the opposite was observed for both fungi F18 and F20. A molecular formula of the protonated ion of enniatin B1 degradation product could be $\mathrm{C}_{34} \mathrm{H}_{62} \mathrm{~N}_{3} \mathrm{O}_{10}$. 


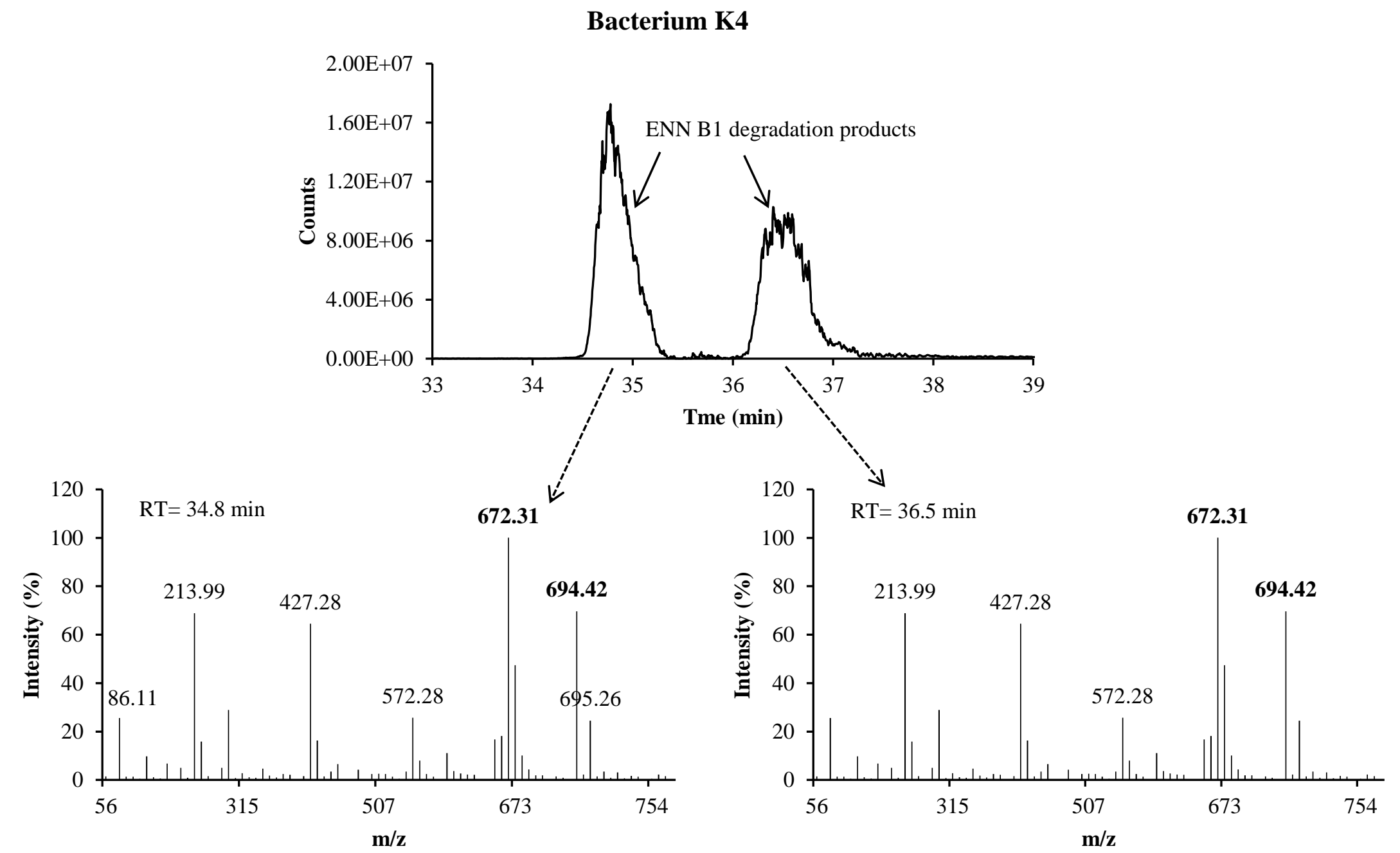

Figure 12: EIC and mass spectra of enniatin B1 degradation product by bacterium $\mathrm{K} 4$ with $[\mathrm{M}+\mathrm{H}]^{+} 672.31$. 


\section{Fungus F18}
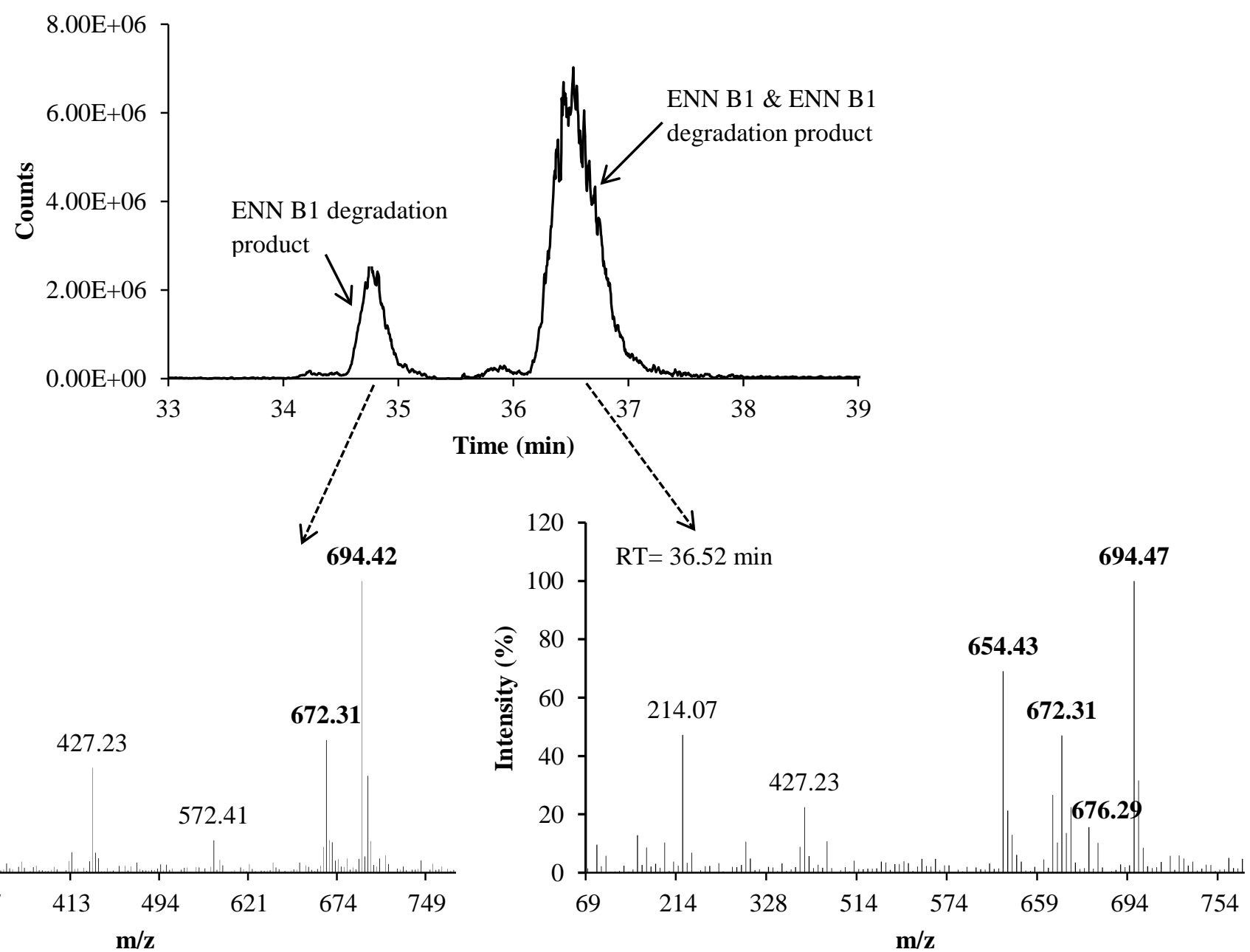

Figure 13: EIC and mass spectra of enniatin B1 degradation product by fungus F18 with $[\mathrm{M}+\mathrm{H}]^{+} 672.31$. 


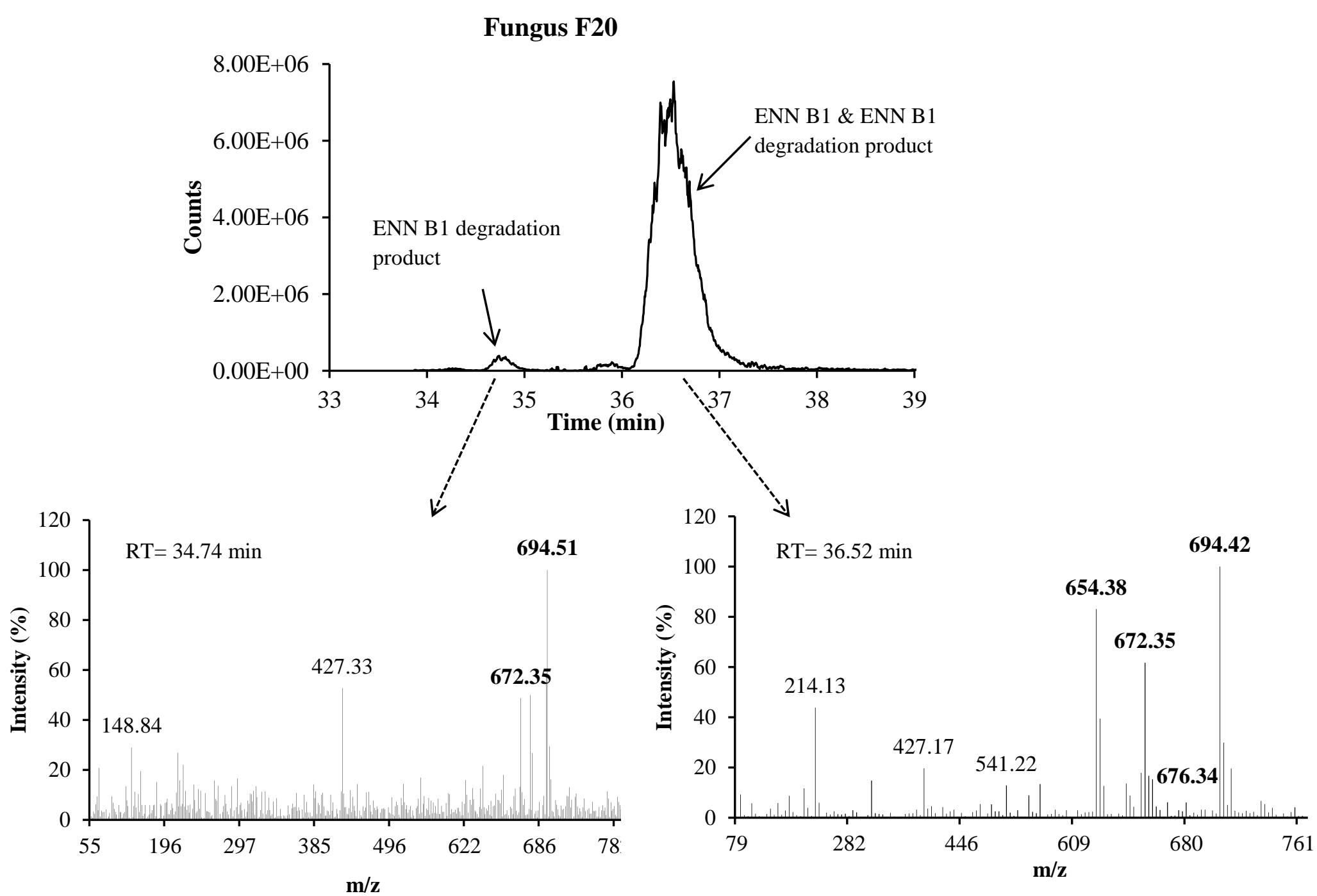

Figure 14: EIC and mass spectra of enniatin B1 degradation product by fungus F20 with $[\mathrm{M}+\mathrm{H}]^{+} 672.35$. 


\subsubsection{Degradation product of enniatin A1}

A product of degradation of enniatin A1 was identified in the culture supernatant of all the 3 microorganisms (K4, F18 and F20). The fragment with a m/z 686.3 corresponded to the molecular weight of the protonated ion of enniatin A1 degradation product and was represented by enniatin A1 with the addition of one molecule of water. The fragment with the $\mathrm{m} / \mathrm{z} 708.5$ represented the sodium adduct of enniatin A1 degradation product. The presence of this compound was confirmed by the extracted ion chromatogram (EIC) presented in Figures 15, 16 and 17. The EIC obtained after the LC-MS showed two peaks for bacterium K4 (Figure 15) and fungus F18 (Figure 16), having the same mass spectrum, while only one peak is observed for the fungus F20 (Figure 17). For K4 and F18, the first peak was more hydrophilic than enniatin A1 due to a lower retention time $(35.9 \mathrm{~min})$ compared to that of enniatin A1 (37.4 min). The second peak had a retention time of $37.4 \mathrm{~min}$ which is comparable to that of enniatin A1. The mass spectrum of the second peak was dominated by the fragments with $[\mathrm{M}+\mathrm{H}]^{+}$and $[\mathrm{M}+\mathrm{Na}]^{+}$at $\mathrm{m} / \mathrm{z} 686.3$ and 708.5 respectively but there were also some fragments with $[\mathrm{M}+\mathrm{H}]^{+}$and $[\mathrm{M}+\mathrm{Na}]^{+}$at $\mathrm{m} / \mathrm{z} 668.4$ and 690.4 respectively corresponding to the protonated ion and the sodium adduct ion of enniatin A1. For both fungi F18 and F20, the sodium adduct ion of the enniatin A1 degradation product was dominant compared to the protonated ion. While for the bacterium $\mathrm{K} 4$, the first peak representing enniatin A1 degradation product was dominated with the protonated ion compared to the sodium adduct ion. The molecular formula of the protonated ion corresponding to the enniatin A1 degradation product is $\mathrm{C}_{35} \mathrm{H}_{64} \mathrm{~N}_{3} \mathrm{O} 10$. 


\section{Bacterium K4}
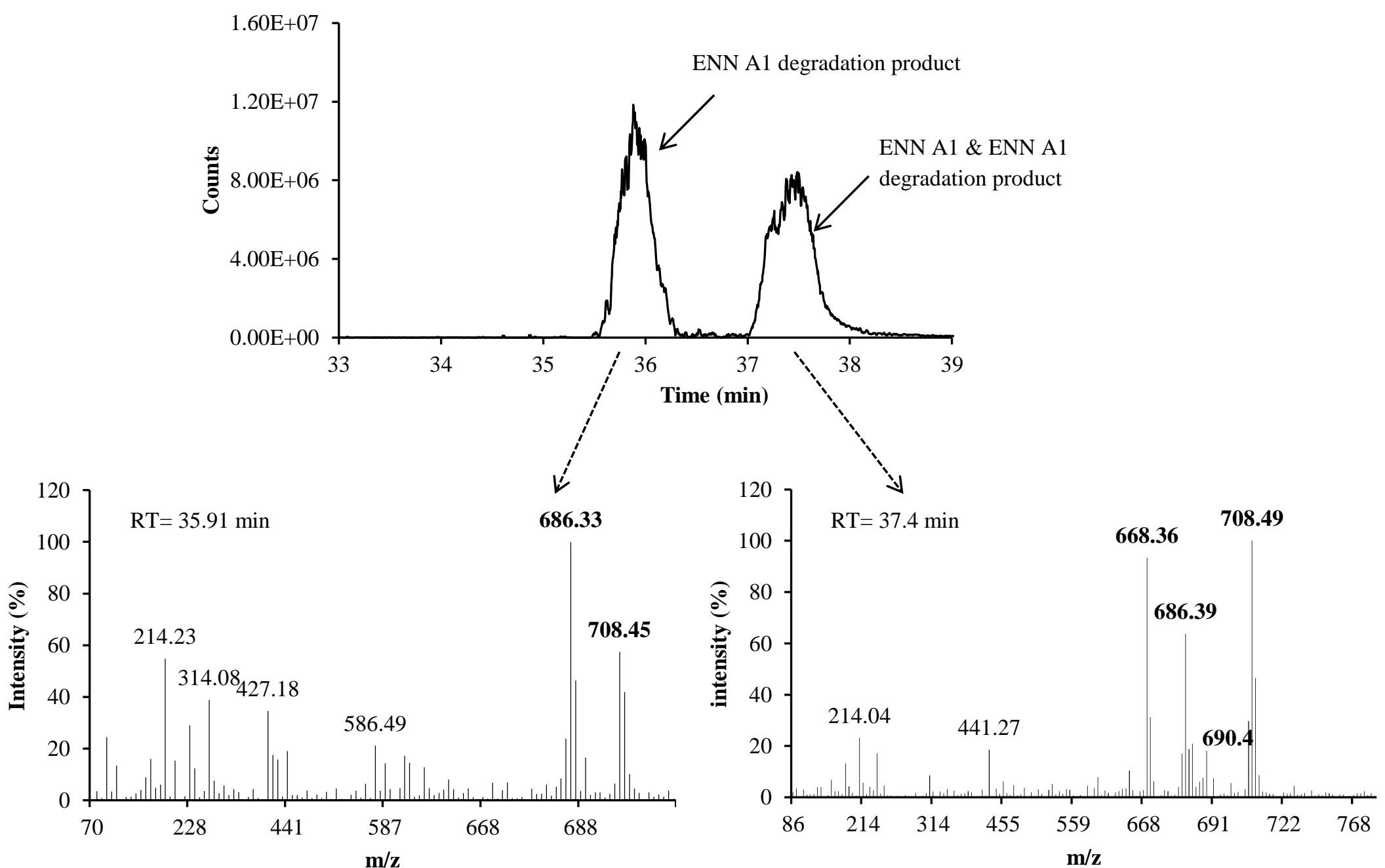

Figure 15: EIC and mass spectra of enniatin A1 degradation product by bacterium $\mathrm{K} 4$ with $[\mathrm{M}+\mathrm{H}]^{+} 686.33$ and 686.39 . 


\section{Fungus F18}
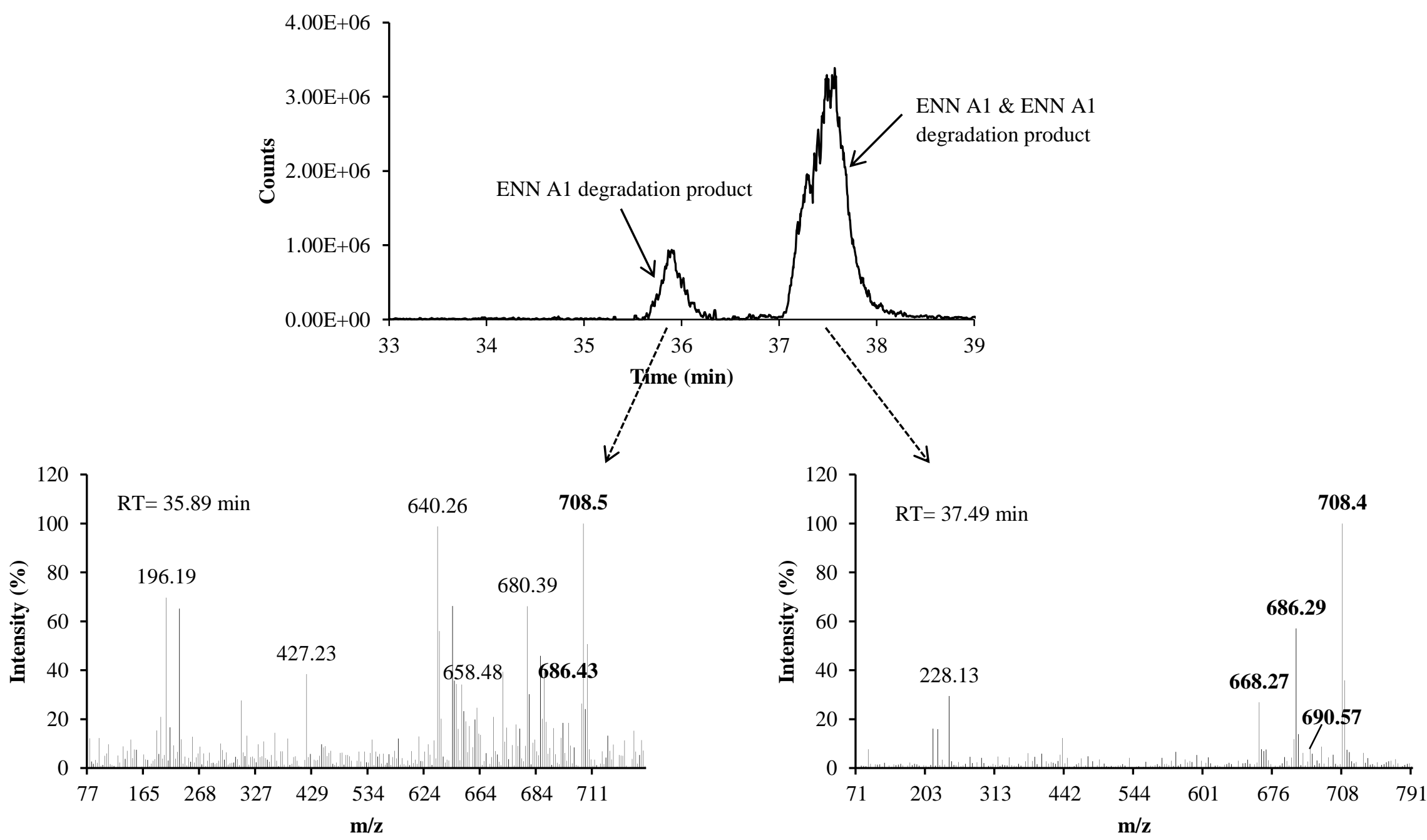

Figure 16: EIC and mass spectra of enniatin A1 degradation product by fungus F18 with $[\mathrm{M}+\mathrm{H}]^{+} 686.29$ or 686.43. 


\section{Fungus F20}

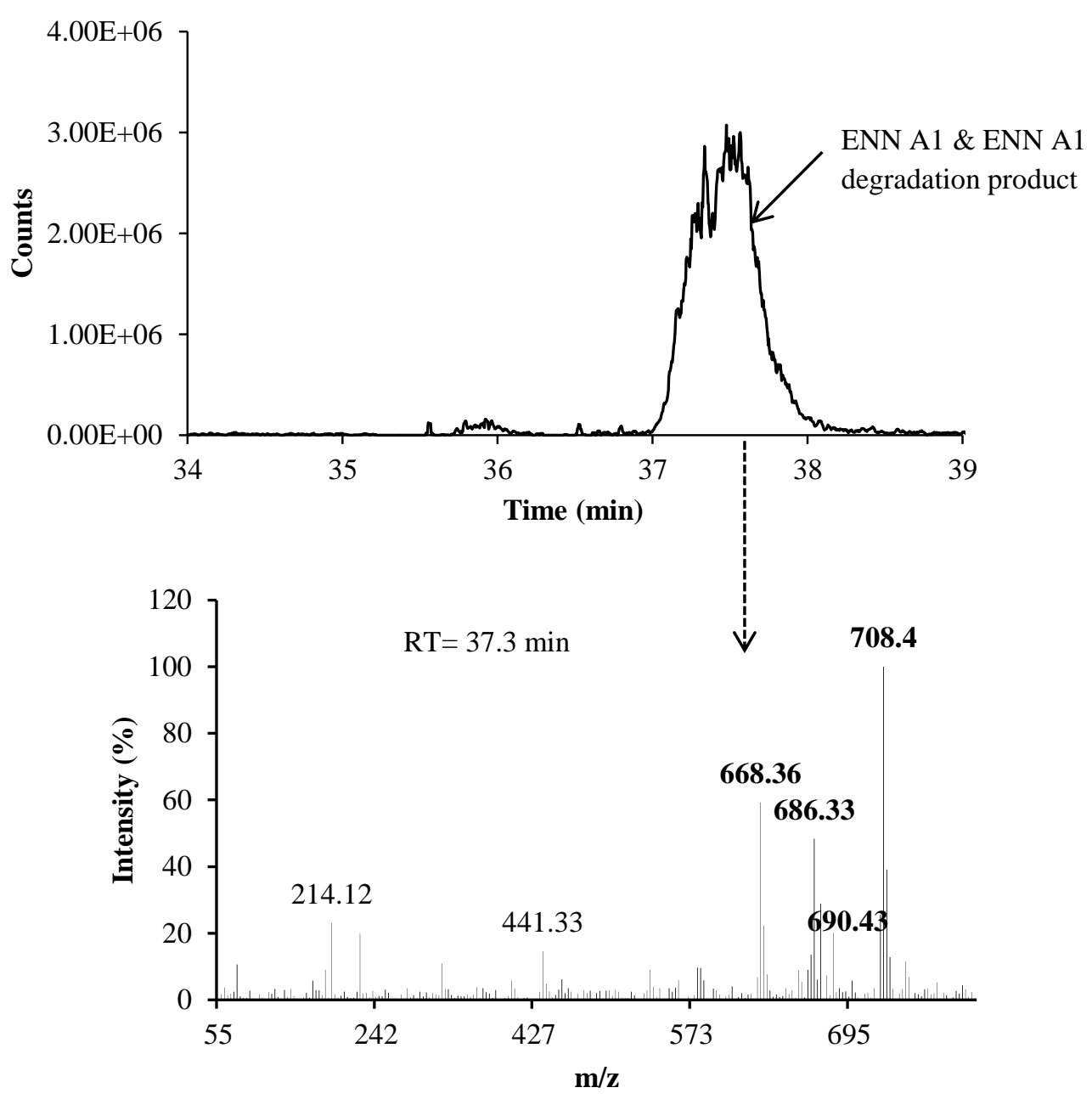

Figure 17: EIC and mass spectra of enniatin A1 degradation product by fungus F20 with $[\mathrm{M}+\mathrm{H}]^{+} 686.32$ or 686.33 . 


\subsubsection{Degradation product of enniatin A}

Fragments were found corresponding to the enniatin A degradation products with a protonated ion at $\mathrm{m} / \mathrm{z} 700.3$ and sodium adduct ion at $\mathrm{m} / \mathrm{z} 722.4$, the EIC and the mass spectra corresponding to the degradation products are presented in Figure 18, 19 and 20. For bacterium K4 (Figure 18), two peaks were observed eluting at $36.6 \mathrm{~min}$ and $38.2 \mathrm{~min}$ respectively and with the same mass spectrum. The second peak overlapped with enniatin A. Enniatin A was recognized by the presence of fragments with $[\mathrm{M}+\mathrm{H}]^{+}$and $[\mathrm{M}+\mathrm{Na}]^{+}$at 682.3 and 704.5 with a retention time of $38.4 \mathrm{~min}$. In the culture supernatant of the fungus F20, enniatin A degradation product was present as a single peak overlapping that of enniatin A on the EIC (Figure 20). Contrary to the bacterium K4 and fungus F20, no residual fragment corresponding to enniatin A was observed on the EIC after analysis with LC-MS for the fungus F18, the fragment of the enniatin A degradation product eluted at $38.2 \mathrm{~min}$ (Figure 19). For the 3 microorganisms tested, the sodium adduct ion of the enniatin A degradation product was dominant compared to the protonated ion. The molecular formula of the protonated ion corresponding to the enniatin A degradation product is $\mathrm{C}_{36} \mathrm{H} 66 \mathrm{~N} 3 \mathrm{O} 10$. 


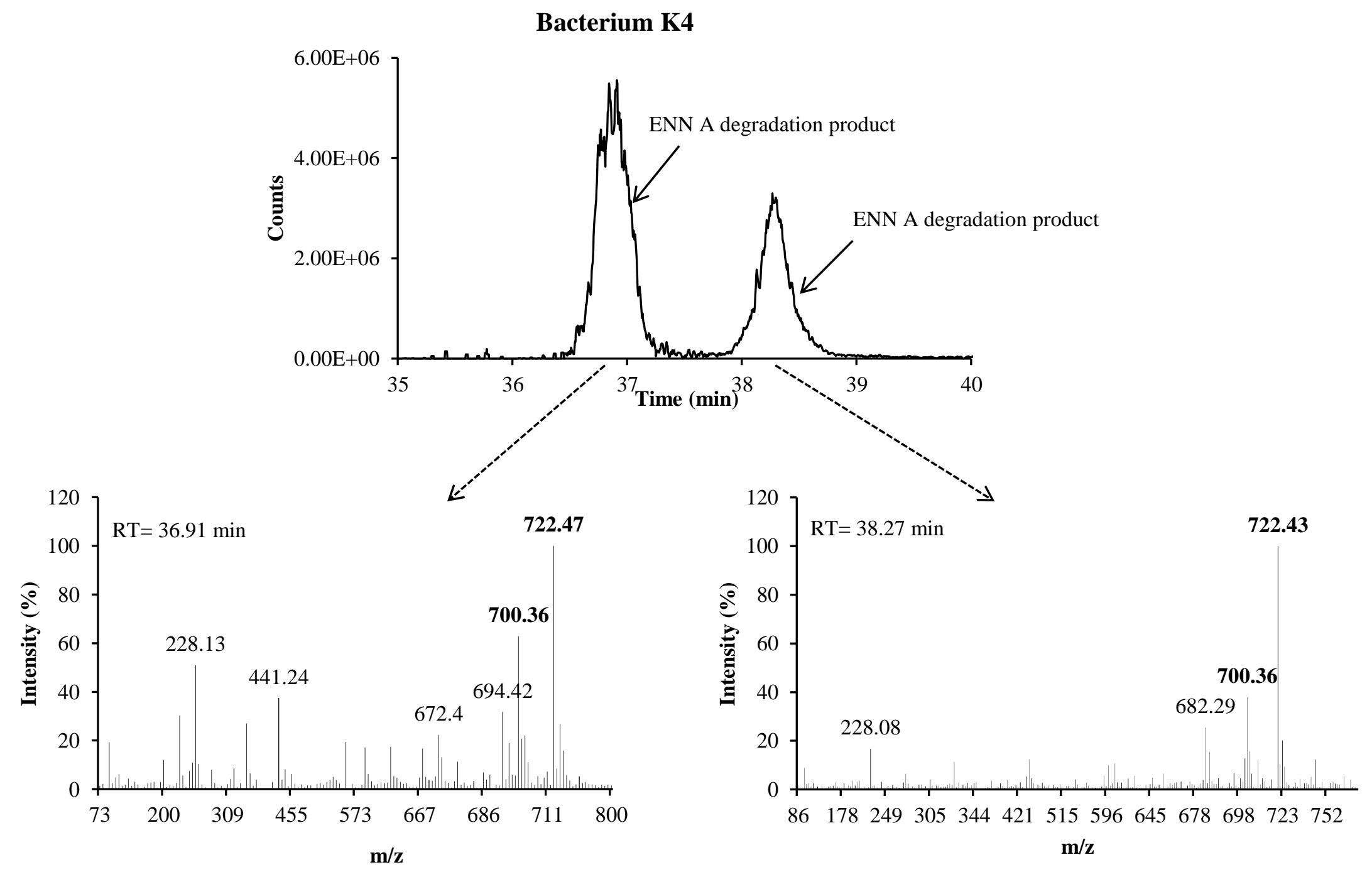

Figure 11: EIC and mass spectra of enniatin A degradation product by bacterium K4 with $[\mathrm{M}+\mathrm{H}]^{+} 700.36$. 
Fungus F18

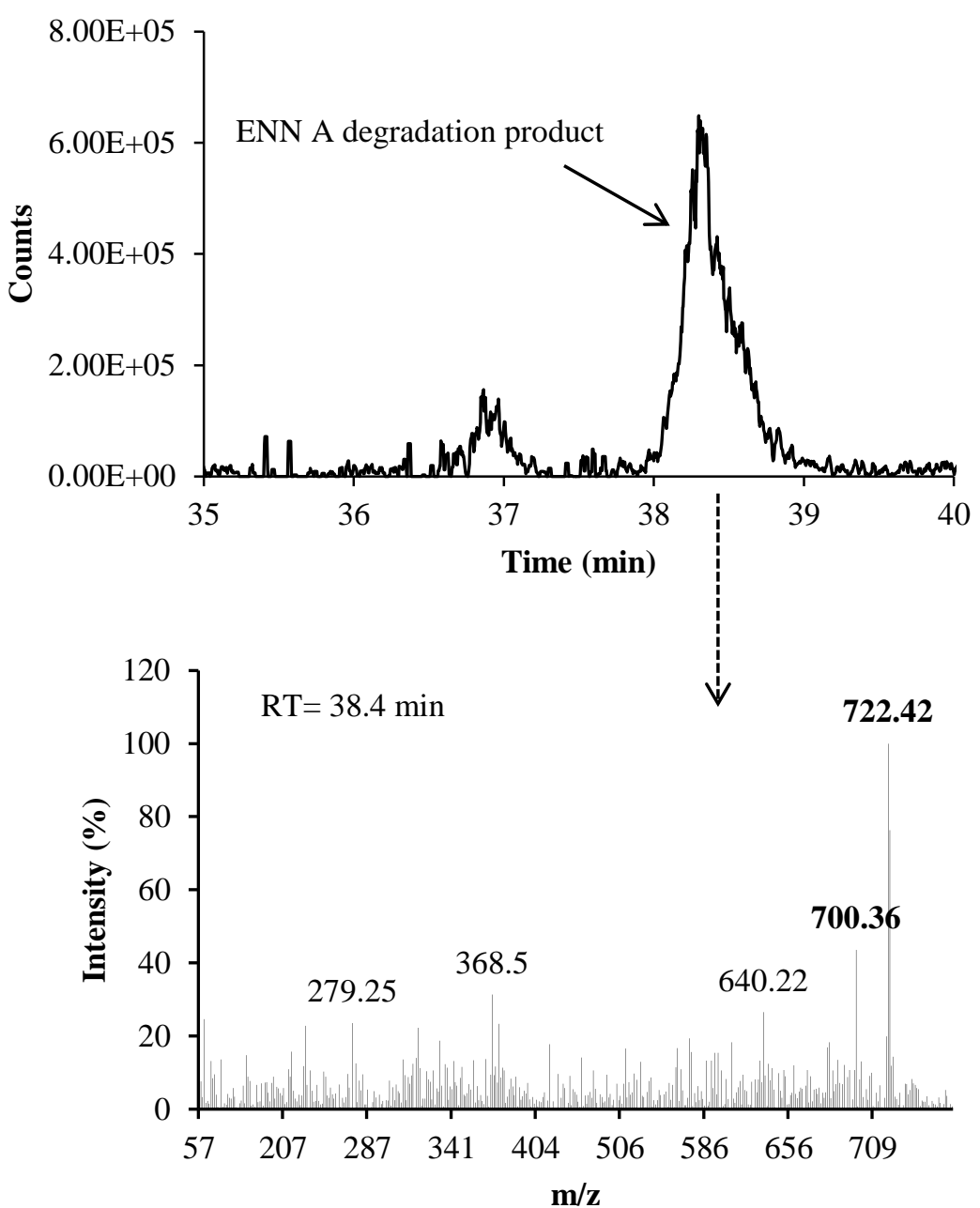

Fungus F20

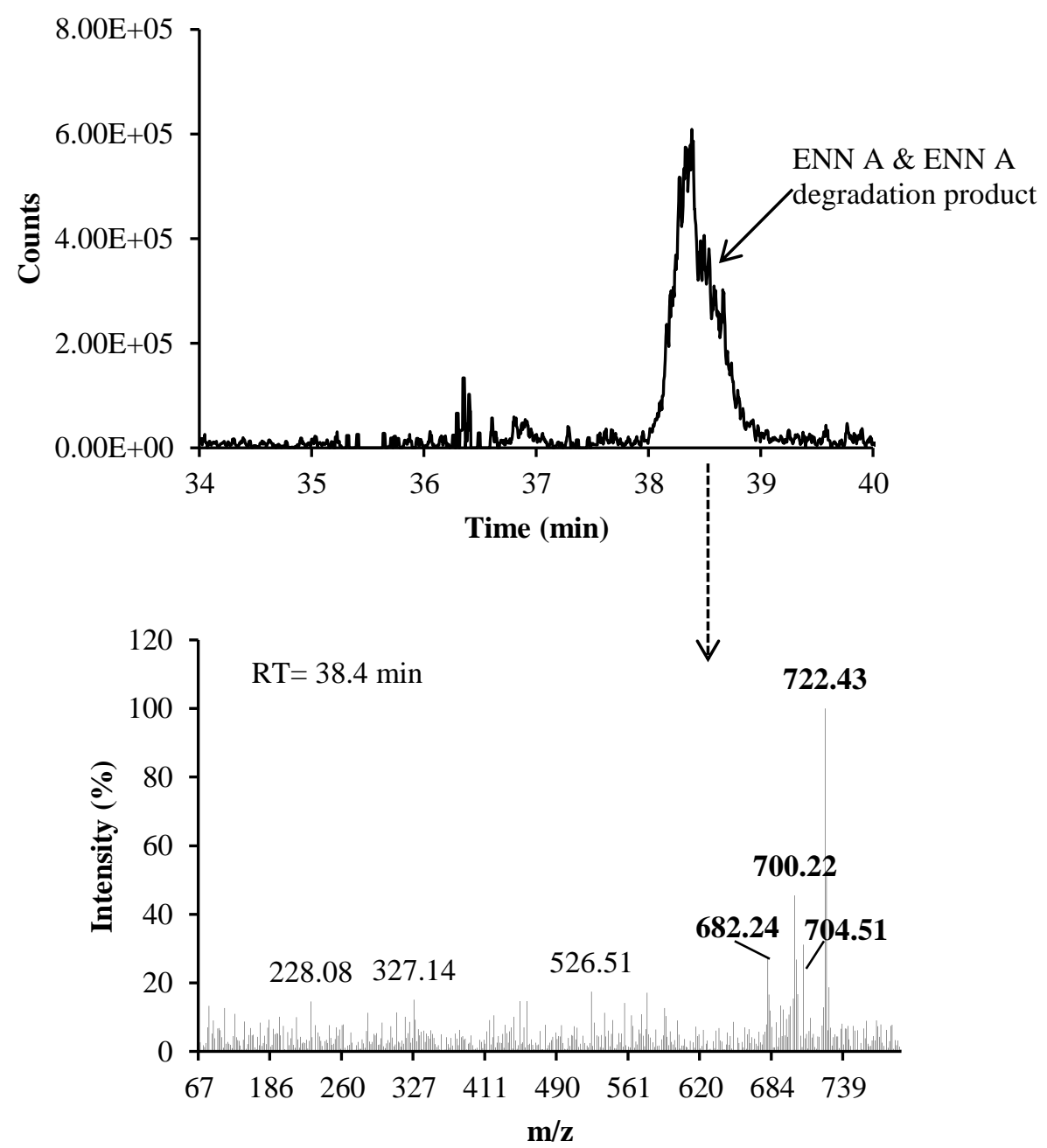

Figure 14: EIC and mass spectra of enniatin A degradation product by fungi F18 and F20 with $[\mathrm{M}+\mathrm{H}]^{+} 700.36$ or 700.22 . 


\section{Discussion}

\subsection{Selection of microorganisms from mixed cultures}

This section of the study was conducted in order to isolate some microorganisms capable of utilizing enniatin as unique carbon source, by incubating a mixed culture from diverse sources in a minimal medium containing enniatin (final concentration $1 \mathrm{mg} / \mathrm{mL}$ ). After observation of the wells of the microtiter plate under binocular microscopes, signs of growth of the microorganisms through the presence of mycelia or turbidity and colloidal solution were noted, the disappearance of enniatin crystals was also observed. Enniatins are a class of compounds which form crystals as shown in the previous chapter of this thesis and also shown by Burmeister and Plattner (1987), Strongman et al. (1988), Blais et al. (1991), Richter et al. (2014). The property of beauvericin to form crystals has been exploited by Duvick and Rood (2000) in the detoxification process of the mycotoxin beauvericin which is structurally similar to enniatin. Beauvericin was incubated with a mineral salt suspension medium and the disappearance of beauvericin crystals was monitored during the process. During our assay, although enniatin crystals disappeared in some wells, no microorganism was isolated. This may be because some microorganisms which are able to grow in the minimal medium containing enniatin as unique carbon source may not grow on the agar plates and the conditions used here, what might have prevented their isolation during these experiments.

\subsection{Sources of isolated microorganisms}

During our study, the strategy used to isolate enniatin-metabolizing microorganisms included first the isolation from mixed cultures of microorganisms able to utilize enniatin as unique carbon and energy source then the isolated microorganisms were screened for enniatin transformation by monitoring the enniatin disappearance and the appearance of new compounds in the culture media by HPLC-UV.

In the literature available, many materials have been used in the search of mycotoxin detoxifying microorganisms. In the case of the present study, the major elements providing bacteria with the ability to grow on media containing enniatin as a unique carbon source were soil, water, grains and nuts in a decreasing order. These results conform to other findings in the literature (Ikunaga et al., 2011; Yi et al., 2011; Tan et al., 2014) which confirms the richness of soil in terms of the microorganisms possessing mycotoxin degrading properties. 
The use of grains, nuts and cereal based products as sources of microorganisms for the detoxification of enniatin was based on the idea that, microorganisms having the ability to degrade enniatins might be present in the same compound from which high enniatin concentrations have been found. This was verified by the high number of microorganisms isolated from these sources. A typical example of this hypothesis is the use of wheat kernels infested with $F$. graminearum to isolate Nocardia globulera strains able to detoxify beauvericin (Duvick and Rood, 2000).

Water samples also provided several bacteria able to grow on media containing enniatin as a unique carbon source. Although no further activity was detected using HPLC in this study, water constitutes a suitable source of bacteria and yeast in terms of mycotoxin degradation. For example Zhu et al. (2015) demonstrated the ability of the yeast Rhodosporidium paludigenum isolated from South East China Sea to degrade patulin into a less toxic compound.

\subsection{HPLC-UV chromatograms and enniatin reduction in culture media}

In general a reduction of enniatin concentrations in the cultures of all isolated microorganisms was observed, but no new signal was observed for most microorganisms after analysis with HPLC-UV of those cultures. Völkl et al. (2004) have described an adsorption of deoxynivalenol into the cell wall of the microorganism; this was verified by lysing the cell wall with proteinase $\mathrm{K}$ and SDS. Although this was not tested in the present study, this might be a plausible explanation of the fact that enniatin concentrations were reduced in some cases but no new signal of metabolization of enniatin by the microorganism was observed.

The order of reduction of enniatin in the culture media of tested microorganisms was in general enniatin $\mathrm{A}, \mathrm{A} 1, \mathrm{~B} 1$ and $\mathrm{B}$ in a decreasing order, this might be due to the initial proportion of enniatin used (the mixture of enniatin contained about $77 \%$ enniatin $\mathrm{B}, 17 \%$ enniatin B1, 6\% enniatin A1 and $0.3 \%$ enniatin A); there is no other obvious explanation of this trend where enniatin A is utilized most compared to enniatin B.

The chromatograms of the samples containing the bacterium K4 or the fungi F18 and F20 showed different patterns compared to those of the control samples, also new peaks having a lower retention time were observed. After comparison of the HPLC-UV chromatograms of bacterium K4 and fungi F18 and F20 when incubated in medium containing enniatin and those when incubated in medium without enniatin, they were assumed to transform enniatins 
into new products. The new peaks suggested the presence in the culture media of new less apolar compounds resulting from the degradation of enniatins used by the microorganisms present in the media. Since the chromatogram pattern of bacterium K4 was identical when it was incubated either in MM or in MMGP, the degradation of enniatins by bacterium K4 seemed not to be affected by the composition of the media used and was independent of the presence of another carbon source such as glucose and tryptone present in the medium MMGP and absent in the medium MM. On the contrary, fungus F20 and F18 seemed to manifest a higher change when enniatins are present in the media when incubated in MMGP compared to MM, the presence of glucose and peptone in MMGP seemed to favor the ability of the fungi to transform enniatins. Due to the limitation in terms of precision of the HPLC$\mathrm{UV}$, no further information such as the possible compound corresponding to those new signals could be extracted from the results; therefore the samples were analyzed using HPLCMS.

\subsection{Identification of degradation products using HPLC-MS}

Experiments in this section were conducted to find the enniatin degradation products resulting from the action of the bacterium K4 and fungi F18 and F20 incubated in MMGP with enniatin A, A1, B1 and B using HPLC-MS and to deduce the degradation reactions and the possible formula of the products. The procedure to identify the degradation products during our study involved the analysis of the cultures of the supernatants of the microorganisms incubated in MMGP containing enniatin, compared to the microorganisms incubated in MMGP without enniatin. When new signals in the treated samples were found, the mass to charge was determined and the detoxification reaction was postulated.

Four degradation products were confirmed, resulting from the hydrolysis of enniatins B, B1, A1 and A. All the four degradation products found in the culture supernatant of bacterium K4 appeared as double peaks in the EIC, the two peaks possessed the identical mass spectrum. This led to the idea that the hydrolysis occurred at 2 different sites in ring of cyclic structure of enniatin, leading to two compounds having the same molecular mass and formula but different chemical structures. In EIC of the HPLC-MS analysis the samples of the fungus F18, the degradation products of enniatins B, B1 and A1 showed also two peaks having the same mass spectrum, while the degradation product of enniatin A was present as a single peak. As for the fungus F20, the degradation products of enniatins A and A1 were single peaks in the EIC while the ones of enniatins B and B1 were present as double peaks with the same mass 
spectra. This highlights the divergence of the action of the bacterium K4 and fungi F18 and F20 towards enniatin degradation.

Few references are available in the scientific literature concerning enniatin degradation by microorganisms and their degradation products. Roig et al. (2013) identified three enniatin degradation products after incubating several strains of Lactobacillus and Saccharomyces in the liquid medium De Man Rogosa Sharpe (MRS) and potato dextrose broth respectively for 48 hours. The degradation products were characterized using liquid chromatography coupled to the mass spectrometry detector in tandem (LC-MS/MS). They were found as enniatin B with the loss of a structural component represented by the hydroxyisovaleric acid, the enniatin B with the loss of two molecules of water and also of a methyl group and the enniatin B1 with the loss of a structural component as the valine unit. The degradation of enniatins B and B1 was reported by Meca et al. (2014). In this case, the degradation was done by a Bacillus subtilis strain incubated in tryptic soy broth for 48 hours, and then the bioactive compounds were analyzed using liquid chromatography coupled to mass spectrometry detector (LC-MS). The degradation products resulted from the loss of hydroxyisovaleric acid unit in the structure of enniatins B and B1.

The process of hydrolysis of enniatins demonstrated in this study is similar to the process of detoxification of the mycotoxin zearalenone (ZEN) mechanisms demonstrated by Kakeya et al. (2002), due to the cleavage of the lactone rings from the backbone and therefore transforming ZEN into a far less potent compound. 


\section{Conclusion}

The present study revealed the following points:

- In total 114 bacteria and 34 fungi isolated from various sources were able to grow on a minimal medium containing enniatins as unique carbon source.

- Soil, water, grains and nuts were the main sources of bacteria, while nuts and grains were the main sources of fungi able to grow on a minimal medium containing enniatins as unique carbon source.

- A reduction of enniatins was observed when the above mentioned microorganisms were incubated in MM and MMGP, the tendency of reduction varied depending on the microorganism and the media used. Enniatin reduction was higher in MMGP compared to MM for fungi, while bacteria showed a comparable rate of reduction in both media.

- Out of the 88 bacteria and 23 fungi tested as single culture for enniatin degradation, one bacterium and two fungi were able to metabolize enniatins when incubated in MMGP and/or MM leading to the formation of new compounds. Reduction of enniatins in the culture media of other microorganisms was presumably due to adsorption into the cell wall.

- The bacterium K4 and one fungus F18 able to degrade enniatins were isolated from a wheat field soil sample, while the other fungus F20 was isolated from hazel nut kernels.

- Four enniatin degradation products were identified using HPLC-MS.

- Enniatin degradation products had protonated ions with $\mathrm{m} / \mathrm{z}$ 658, 672, 686 and 700 for enniatin B, B1, A1 and A respectively. It was assumed that enniatin degradation products resulted from the hydrolysis of an ester or an amide bond in the cyclic ring of enniatins.

This is the first report which demonstrates the capacity of a bacterium K4 and fungi F18 and F20 to degrade enniatin B, B1, A1 and A forming four degradation products.

The deep characterization of the degradation compounds in a kinetics assay is ongoing using Ultra Performance Liquid Chromatography (UPLC). Further investigations will focus on the incubation of single enniatin variants with the detoxifying microorganisms, the monitoring of the degradation process under different parameters and the purification of the degradation compounds. The structure of the detoxification product should be confirmed using nuclear magnetic resonance (NMR). Moreover, the toxicological parameters of the purified degradation compounds should be evaluated. 


\section{Acknowledgement}

We acknowledge Dr. Kirstin Feussner (Department of Plant Biochemistry, Albrecht-vonHaller Institute for Plant Sciences, Georg-August University, Göttingen) who carried out the UPLC analysis for identification of enniatin degradation products used for a presentation at the $38^{\text {th }}$ mycotoxin workshop.

\section{References}

Bhatnagar, D., Lillehoj, E.B., Bennett,J.W. (1991): Biological detoxification of mycotoxins, In: Mycotoxins and animal foods, Smith, J.E., Henderson, R.S., ed. CRC Press, Boca Raton, FL, 816-826.

Blais, L. A., Apsimon, J. W., Blackwell, B.A, Greenhalgh, R., Miller, J. D. (1992): Isolation and characterization of enniatins from Fusarium avenaceum DAOM 196490, Canadian Journal of Chemistry, 70 (5):1281- 1287.

Braun, R. (1960): Ueber Wirkungsweise und Umwandlung der Fusarinsaeure, Phytopatologie Zeitschrift, 39: 196-241.

Burmeister, H.R., Plattner, R.D. (1987): Enniatin production by Fusarium tricinctum and its effect on germinating wheat seeds, Phytopathology, 77: 1483-1487.

ChemCalc (2013): A building block for tomorrow's chemical infrastructure. Patiny, Luc; Borel, Alain Journal of Chemical Information and Modeling. DOI: 10.1021/ci300563h.

Cheng, B.C., Wan, C.X., Yang, S.L., Yu, H.Y., Wei, H., Liu, J.S., Tian, W.H., Zeng, M. (2010): Detoxification of deoxynivalenol by Bacillus strains, Journal of Food Safety 30: 599614.

Ciegler, A., Lillehoj, E.B., Peterson, R.E., Hall, H.H. (1966): Microbial detoxification of aflatoxin, Applied Microbiology, 14: 934-939.

Duvick, J., Rood, T. A. (2000): Beauvericin detoxification methods using bacteria, US 6126934 A. 
EFSA panel on contaminants in the food chain (2014): Scientific opinion on the risks to human and animal health related to the presence of beauvericin and enniatins in food and feed, EFSA journal, 12(8): 3802.

Firáková, S., Turdíkova, M.S., Liptaj, T., Prónayova, N., Bezákova, Proksa, B. (2008): Enniatins produced by Fusarium dimerum, an endophytic fungal strain, Pharmazie, 63: 539541.

Gäumann, E., Naef-Roth, S., Kern, H. (1960): Zur phytotoxischen Wirksamkeit der Enniatine, Phytopathologie Zeitung, 40: 45-51.

Hackbart, H. C. S., Machado, A. R., Christ-Ribeiro, A., Prietto, L., Badiale-Furlong, E. (2014): Reduction of aflatoxins by Rhizopus oryzae and Trichoderma reesei, Mycotoxin Research, 30(3): 141-149.

Hult, K., Teiling, A., Gatenbeck, S. (1976): Degradation of ochratoxin A by a ruminant, Applied Environmental Microbiology, 32: 443-444.

Ikunaga, Y., Sato, I., Grond, S., NUmaziri, N., Yoshida, S., Yamaya, H. et al. (2011): Nocardioides sp. strain WSN05-2, isolated from a wheat field, degrades deoxynivalenol, producing the novel intermediate 3-epi- deoxynivalenol, Applied Microbiology, 89: 419- 427.

Jestoi, M., Rokka, M., Yli-Mattila, T., Parikka, P., Rizzo A., Peltonen, K. (2004): Presence and concentrations of the Fusarium-related mycotoxins beauvericin, enniatins and moniliformin in finnish grain samples, Food Additives and Contaminants, 21(8):794-802.

Kakeya, H., Takahashi-Ando, N., Kimura, M., Onose, R., Yamaguchi, I., Osada, H. (2002): Biotransformation of the mycotoxin zearalenone, to a non-oestrogenic compound by the fungal strain of Clonostachys sp. Bioscience, Biotechnology and Biochemistry, 66: 27232726.

Karlovsky, P. (1999): Biological Detoxification of Fungal Toxins and its Use in Plant Breeding, Feed and Food Production, Natural Toxins, 7: 1-23.

Mahnine, N, Meca, G., Elabidi, A., Fekhaoui, M., Saoiabi, A et al. (2011): Further data on the levels of emerging Fusarium mycotoxins enniatins (A, A1, B, B1), beauvericin and fusaproliferin in breakfast and infant cereals from Morocco, Food Chemistry, 124: 481-485. 
Meca, G., Roig, M., Ferrer, E., Mañes, J. (2014): Degradation of the bioactive compounds enniatins A, A1, B, B1 employing different strains of Bacillus subtilis, Journal of Food Processing and Technology, 5: 334.

Meca, G., Sospedra, I., Valero, M.A., Mañes, J., Front, G., Ruiz, M.J. (2011): Antibacterial activity of the enniatin B, produced by Fusarium tricinctum in liquid culture, and cytotoxic effects on Caco-2 cells, Toxicology Mechanisms and Methods, 21(7): 503-512.

Meyer, O. and Sch1egel, G. H. (1983): Biology of aerobic carbon monoxide- oxidizing bacteria, Annual Review of Microbiology, 37: 277-310.

Moffat, A.C. (1986): Clarke's isolation and Identification of Drugs, The pharmaceutical Press, London.

Monti, S.M., Fogliano, V., Logrieco, A., Ferracane, R., Ritieni, A. (2000): Simultaneous determination of beauvericin, enniatins and fusaproliferin by high performance liquid chromatography, Journal of Agricultural Food Chemistry, 48: 3317-3320.

Richter, L., Wanka, F., Boecker, S., Storm, D., Kurt, T., Vural, Ö., Süßmuth, R., Meyer, V. (2014): Engineering of Aspergillus niger for the production of secondary metabolites, Fungal Biology and Biotechnology, 1:4.

Roig, M., Meca, G., Ferrer, E., Manes, J. (2013): Reduction of the enniatins A, A1, B, B1 by an in vitro degradation employing different strains of probiotic bacteria: Identification of degradation products by LC- MS- LIT, Toxicon, 70: 44-53.

Sebastià, A., Meca, G., Soriano, J.M., Mañes, J. (2011): Antibacterial effects of enniatins J 1 and $\mathrm{J}_{3}$ on pathogenic and lactic acid bacteria, Brief Communication, Food and Chemical Toxicology, 49: 2710-2717.

Serrano, A.B., Meca, G., Font, G., Ferrer, E. (2013): Degradation study of enniatins by liquid chromatography-triple quadrupole linear ion trap mass spectrometry, Food Chemistry, 141: 4215-4225.

Shemyakin, M.M., Ovchinnikov, Y.A., Ivanov, N.T., Antonov, V.K., Vinogradova, E.I., Shkrob, A.M., Malenkov, G.G., Evstratov, A.V., Laine, I.A., Melnik, E.I., Ryabova, I.D. 
(1969): Cyclodepsipeptide as chemical tools for studying ionic transport through membranes, Journal of Membrane Biology, 1: 402-430.

Sørensen, J.L., Nielsen, K.F., Rasmussen, P.H., Thrane, U. (2008): Development of a LCMS/MS method for the analysis of enniatins and beauvericin in whole fresh and in ensiled maize samples, Journal of Agricultural and Food Chemistry, 56(21): 10439-43.

Strongman, D.B., Strunz, G.M., Giguère, P., YU, C.-M., Calhoun, L. (1988): Enniatins from Fusarium avenaceum isolated from balsam fir foliage and their toxicity to spruce budworm larvae, Choristoneura fumiferana (Clem.) (Lipidoptera: Tortricidae), Journal of Chemical Ecology, 14 (3): 753-764.

Tan, H., Hu, Y., He, J., Wu, L., Liao, F., Luo, B., et al. (2014): Zearalenone degradation by two Pseudomonas strains from soil, Mycotoxin Research, 30(4): 191-196.

Tartof, K.D. and Hobbs, C. A. (1987): Improved media for growing plasmid and cosmid clones, Bethesda Research Laboratory Focus, 9: 12-17.

Tolosa, J, Font, G. Mañes, J, Ferrer, E. (2013): Nuts and dried fruits: Natural occurrence of emerging Fusarium mycotoxins, Food control, 33: 215-220.

Uhlig, S., Ivanova, I. (2004): Determination of beauvericin and four other enniatins in grain by liquid chromatography-mass spectrometry. Journal of Chromatography A, 2: 173-178.

Völkl, A., Vogler, B., Schollenberger, M., Karlovsky, P. (2004): Microbial detoxification of mycotoxin deoxynivalenol, Journal of basic microbiology, 44 (2): 147- 156.

Yi, P.-J., Pai, C.-K., Liu, J.-R. (2011): Isolation and characterization of a Bacillus licheniformis strain capable of degrading zearalenone, World Journal of Microbiology and Biotechnology, 27: 1035-1043.

Yli-Mattila, T., Paavanen-Huhtala, S., Jestoi, M., Parikka, P., Hietaniemi, V., Gagkaeva, T., Sarlin,T., Haikara, A., Laaksonen, S. and Rizzo, A. (2008): Real-time PCR detection and quantification of Fusarium poae, F.graminearum, F. sporotrichioides and F. langsethiae in cereal grains in Finland and Russia, Archives of Phytopathology and Plant Protection, 41(4): 243-260. 
Zhu, R., Feussner, K., Wu, T., Yan, F., Karlovsky, P., Zhend, X. (2015): Detoxification of mycotoxin patulin by the yeast Rhodosporidium paludigenum, Food Chemistry, 179: 1-5.

Zinedine, A., Meca, G., Mañes, J., Font, G., (2011): Further data on the occurrence of Fusarium emerging mycotoxins enniatins (A, A1, B, B1), fusaproliferin and beauvericin in raw cereals commercialized in Morocco, Food control, 22: 1-5. 


\section{Appendix}
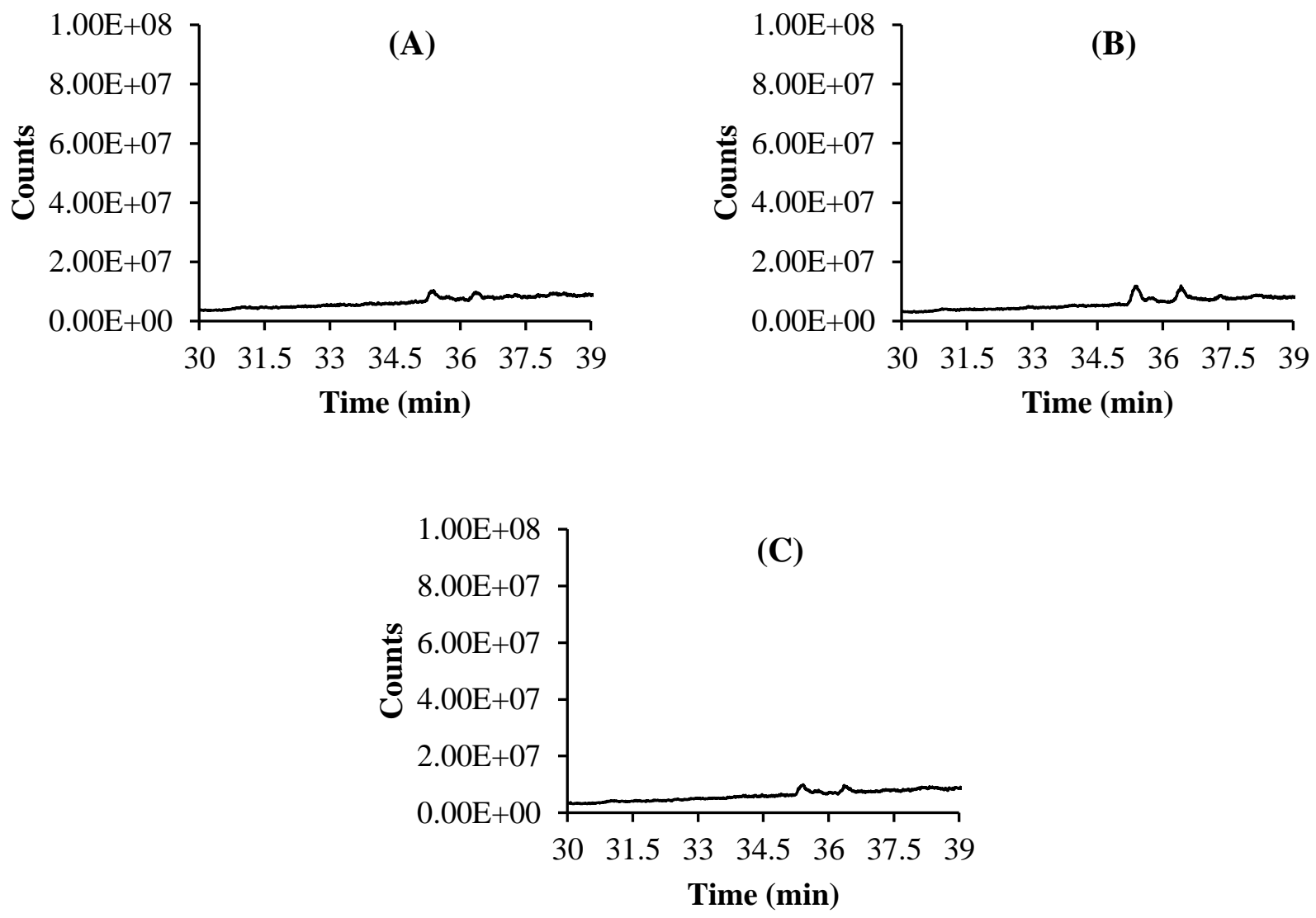

Figure i: Total ion chromatograms of bacterium K4 (A), fungus F18 (B) and fungus F20 (C) incubated in MMGP for 3 days (K4) and 5 days (F18, F20). 


\title{
Chapter 4
}

\section{Identification of a bacterium and two fungi capable of degrading enniatins}

\author{
Rosine G. Suchfort, Petr Karlovsky* \\ Molecular Phytopathology and Mycotoxin Research, Georg-August-University Göttingen, \\ Grisebachstr. 6, 37077 Göttingen, Germany \\ *Corresponding author: pkarlov@gwdg.de
}

\begin{abstract}
Within the framework of finding some microorganisms able to degrade enniatins as reported in the previous chapter, this study was conducted in order to identify pure unknown cultures of a bacterium and two fungi isolated previously based on their ability to degrade enniatins. The identification process was made initially based on the cultural, morphological and biochemical (for the bacterium) characteristics of the microorganisms. The species of the microorganisms were confirmed based on the 16S rRNA gene sequence for the bacterium and the sequences of the Internal Transcribed Spacer 1 (ITS1), 5.8S subunit of the ribosomal RNA and Internal Transcribed Spacer 1 (ITS2) regions for the fungi. The bacterium isolated from a soil sample collected in a wheat field was identified as Bacillus licheniformis, while the fungi isolated from Hazel nuts and a wheat field soil sample (same source as the bacterium) were identified as Clonostachys rosea and Acremonium strictum respectively.
\end{abstract}

Keywords: Enniatin, Bacillus licheniformis, Clonostachys rosea, Acremonium strictum, morphological and molecular characterization, ITS1, ITS2, 16S rRNA. 


\section{Introduction}

Bacteria, yeast and fungi have recently been used for degradation of mycotoxins by acting as biotransforming agents. These microorganisms are active in the degradation of particular mycotoxins or class of mycotoxins by transforming them into less toxic compounds (Boudergue et al., 2009). The isolation of microorganisms which have mycotoxin detoxification properties generally involves the use of enrichment culturing and/or screening strategies to obtain mixed or pure cultures (Karlovsky, 1999). Generally random testing of unknown cultures is used and the taxonomy of active microorganisms is made after detecting any activity. For instance strains of Nocardia globulera have shown activity in the detoxification of beauvericin -an enniatin structurally identical mycotoxin- both in vitro and in beauvericin contaminated corn, the bacteria were isolated from wheat kernel infested with F. graminearum (Duvick and Rood, 2000). According to several studies, soil is a source that has provided numerous microorganisms able to degrade mycotoxins. Bacterium Nocardioides sp. strain WSN05-2 isolated from a wheat field soil was able to degrade deoxynivalenol into an intermediate 3-epi- deoxynivalenol (Ikunaga et al., 2011). Four different soil types including sandy, loamy, gyttja and moldy soil were used as a source of microorganisms for the degradation of zearalenone (ZEN). In vitro degradation of ZEN was efficiently achieved by two Pseudomonas strains namely Pseudomonas alcaliphilia TH-C1 and Pseudomonas plecoglossicida TH-L1 (Tan et al., 2014). Likewise, Yi et al. (2011) isolated and characterized a Bacillus licheniformis strain able to degrade zearalenone out of 168 bacterial strains isolated from soil samples. In the three previously mentioned studies, the active bacteria were taxonomically characterized after discovering their detoxification properties, with the combination of biochemical and molecular techniques involving the sequence of the 16S rRNA gene.

On the other hand, some authors have tested taxonomically known microorganisms thought to degrade mycotoxins. Spores, mycelium and culture filtrate of Aspergillus niger strain FS10 removed ZEN in potato dextrose broth, with fluctuating efficiency of $89.56 \%, 43.10 \%$, and $68.16 \%$ respectively (Sun et al., 2014). Nine taxonomically known bacteria (Bifidobacterium longum, Bifidobacterium bifidum, Bifidobacterium breve, Bifidobacterium adolescentes, Lactobacillus rhamnosus, Lactobacillus casei-casei, Lactobacillus ruminis, Lactobacillus casei, Streptococcus thermofilus) and twenty-two strains of Saccharomyces cerevisiae showed a significant reduction of enniatins in vitro (in liquid medium of De Man Rogosa 
Sharpe or in potato dextrose broth) ranging from 5 to $99 \%$ (Roig et al., 2013). Beauvericin reduction in the range of 66 to $100 \%$ has been demonstrated in model solution and in corn flour treated with intracellular raw enzymes of four Saccharomyces cerevisiae (named LO9, YES, A34 and A17) (Meca et al., 2013). Moreover, Meca et al. (2014) tested six known Bacillus subtilis strains in tryptic soy broth contaminated with enniatins (A, A1, B and B1) and recorded a mean reduction of enniatin content of 64 to $99 \%$.

In the previous chapter of this thesis, a bacterium K4, two fungi F18 and F20 were isolated from wheat field soil (K4 and F18) and hazel nut (F20) samples, and showed positive results regarding the degradation of enniatins A, A1, B1 and B . In the present study, bacterium K4, fungi F18 and F20 were phenotypically and genotypically characterized.

For bacterium K4, more specifically we intended to identify:

- The morphological characteristics of the bacterium K4 including: colony size, form, elevation, margin/edges, color, pigment production, opacity, surface, consistency and odor.

- The biochemical characteristics of the bacterium including: Gram stain, catalase test (3\%, 15\%), starch hydrolysis, SIM (indole, motility and sulfide production), nutrient gelatin stab, citrate test and urease test, the bacterial growth at different temperatures and in different media (nutrient broth containing 6.5\% $\mathrm{NaCl}$, sabouraud dextrose agar, nutrient broth, starch agar, pectine agar and casein agar).

- The species of bacterium K4 through the sequence of the 16S rRNA gene.

For fungi F18 and F20 our specific objectives were to determine:

- The optimal growth temperature and $\mathrm{pH}$.

- The morphological characteristics of fungi after visual observation of factors such as colony margin, structure, surface, color (bottom and top), excretion into the medium and presence of oil droplets. And after microscopic observation of fungal structures such as conidia, macroconidia, hyphae, conidiophores, phialides, presence of chlamydospores, septae and clamps connections.

- The species of the fungi using the ITS1, 5.8S RNA ribosomal units and ITS2 regions of the DNA, and translation elongation factor 1-alpha (TEF-1 $\alpha$ ) gene sequence. 


\section{Material and methods}

\subsection{Identification of enniatin-detoxifying fungi}

\subsubsection{Optimal growth temperature and $\mathrm{pH}$}

To examine the optimal growth temperature of the isolated fungi, one agar plug $(5 \mathrm{~mm}$ diameter) of a 5 days old fungus was inoculated on potato dextrose agar (PDA, from Merck, Darmstadt, Germany) and the plates were incubated at $12^{\circ} \mathrm{C}, 16^{\circ} \mathrm{C}, 20^{\circ} \mathrm{C}, 24^{\circ} \mathrm{C}, 28^{\circ} \mathrm{C}$ and $32^{\circ} \mathrm{C}$ respectively during a period of 10 days. The four radial lengths were measured every 24 hours. Each experiment was done in three replicates.

In order to find out the most suitable growth $\mathrm{pH}$ of the isolated fungi, potato dextrose broth (PDB) was prepared and the $\mathrm{pH}$ adjusted to $2.4 ; 3 ; 3.6 ; 4.4 ; 5.6 ; 7 ; 7.5$; and 8 respectively. One agar plug of 5 days old fungi ( $5 \mathrm{~mm}$ diameter) was inoculated into each flask containing $25 \mathrm{~mL}$ autoclaved PDB. The flasks were incubated for 10 days (160 RPM, at optimal growth temperature of each fungus). After 10 days, the cultures were vacuum filtered and the $\mathrm{pH}$ values of the filtrates were recorded. Before filtration, filter papers were dried in an oven overnight at $70^{\circ} \mathrm{C}$, cooled down in a desiccator for 2 hours and weighed prior to filtration. The mycelium on the filter paper was dried in an oven for 24 hours at $70^{\circ} \mathrm{C}$, cooled down in a desiccator for 2 hours and weighed. The weight of mycelium was calculated by deducting the final weight of the mycelium on filter paper from the weight of the filter paper prior to filtration. Each experiment was performed in four replicates.

\subsubsection{Morphological characterization}

\section{Visual description of colony characteristics}

Fungal isolates were grown on PDA (Merck, Darmstadt, Germany) for 7 and 14 days respectively and visual characteristics including colony features (margin, structure, surface, color-bottom and top, excretion into the media and presence of oil droplets were recorded.

\section{Microscopic characteristics of the fungal isolates}

A modified Riddel's simple method of slide culturing (Riddell, 1950) was used for the in situ microscopic observation of the fungi F18 and F20. A glass petri dish containing a filter paper, a U-shaped glass rod and a glass microscope slide were autoclaved at $120^{\circ} \mathrm{C}$ for 20 minutes. 
A block of sabouraud agar of about $7 \times 7 \mathrm{~mm}$ was placed on the microscope slide and the four sides were aseptically inoculated with spores or mycelium fragments from a 7 days old fungus. A sterile cover-slip was placed on the upper surface of the agar block and the filter paper in the petri dish was moisturized with about $3 \mathrm{~mL}$ sterile tap water. The petri dish was incubated at $25^{\circ} \mathrm{C}$ for 48 and 72 hours. After incubation, a drop of lactophenol cotton blue was applied on a clean microscopic slide and the cover-slip was placed mold side down on the lactophenol cotton blue stain slide. The preparations were analyzed using oil immersion and a light microscope (Leica Leitz DMRB) equipped with a color camera (Leica DFC420).

\subsubsection{Molecular characterization of fungi isolates}

The molecular characterization of active fungi was performed using the ITS1 and ITS4 primers to amplify the ITS1 region, 5.8S ribosomal RNA subunit and ITS2 region of the DNA, as well as the amplification a $700 \mathrm{bp}$ region of the translation elongation factor 1-alpha (TEF- $1 \alpha$ ) gene sequence. The DNA of the fungi was extracted, amplified using polymerase chain reaction (PCR) and sequenced.

\section{DNA extraction}

Fungi were grown in PDB for 7 days $\left(120 \mathrm{RPM}, 25^{\circ} \mathrm{C}\right)$ and vacuum filtered. Fungal mycelium was freeze dried in a freeze drier (Christ, Beta 1-8) and stored at $-20^{\circ} \mathrm{C}$. DNA extraction was carried out according to CTAB method (Brandfass and Karlovsky, 2008).

\section{Gel electrophoresis}

In order to check the efficiency of DNA extraction and the purity of extracted DNA, a $3 \mu \mathrm{L}$ DNA sample were mixed with $2 \mu \mathrm{L}$ Blue juice and loaded on a $0.8 \%$ agarose gel and run for 1 hour at $60 \mathrm{~V}$. The gel was stained with ethidium bromide for 10 minutes, then distained in water for 10 minutes and visualized under UV light.

\section{Polymerase chain reaction}

\section{Elongation factors (EF)}

Primers pairs for elongation factor 1 EF1-forwards (5'-ATGGGTAAGGARGACAAGAC-3') and for elongation factor 2 EF2-reverse (5'-GGARGTACCAGTSATCATGTT-3') (O'Donnell et al., 1998) were used for PCR amplification of a part of the translation 
elongation factor $1-\alpha$ gene. The cycling conditions included $95^{\circ} \mathrm{C} 30 \mathrm{sec}, 30 \times 94^{\circ} \mathrm{C} 30 \mathrm{sec}$, $59^{\circ} \mathrm{C} 30 \mathrm{sec}, 72^{\circ} \mathrm{C} 1 \mathrm{~min}$, close loop $72^{\circ} \mathrm{C} 5 \mathrm{~min}$. The extracted DNA was diluted $1 / 100$. A final volume of $25 \mu \mathrm{L}$ was used for PCR and was composed of $10.75 \mu \mathrm{L}$ water, $5 \mu \mathrm{L} 5 \mathrm{x}$ reaction buffer (New England BioLabs), $0.2 \mu \mathrm{L} \mathrm{MgCl}_{2}$ (Bioline), $1.5 \mu \mathrm{L}$ dNTPs $150 \mu \mathrm{M}$ (Bioline, Luckenwalde, Germany), $0.75 \mu \mathrm{L}$ of each primer $0.3 \mu \mathrm{M}$ (Invitrogen by Thermo Fisher Scientific), $0.05 \mu \mathrm{L}$ of One Taq Hotstart DNA polymerase 0.25 U (New England BioLabs, Ipswich, MA, USA) and $6 \mu \mathrm{L}$ of DNA sample. A control PCR without DNA was set up to check for nonspecific amplification. The expected PCR products' size was $700 \mathrm{bp}$.

The checking of the PCR product was performed on agarose gel electrophoresis as described above. The PCR products were purified using isopropanol precipitation and $5 \mu \mathrm{L}$ of the PCR product (144 ng/ $\mu \mathrm{L}$ ) were sent to LCG Genomics GmbH (Ostendstr. 25. TGS Haus 8, 12459 Berlin, Germany) for sequencing.

\section{Internal transcribed spacer (ITS)}

A touch down program was used for the amplification of the internal transcribed spacer region with the primers pairs ITS1 (5'-TCCGTAGGTGAACCTGCGG-3') and ITS4 (5'TCCTCCGCTTATTGATATGC-3') (White et al., 1990). The cycling conditions included $94^{\circ} \mathrm{C} 30 \mathrm{sec}, 10 \times 94^{\circ} \mathrm{C} 30 \mathrm{sec}, 62^{\circ} \mathrm{C}-53^{\circ} \mathrm{C}-1^{\circ} \mathrm{C} /$ cycle $40 \mathrm{sec}, 72^{\circ} \mathrm{C} 1 \mathrm{~min}$, close loop $30 \times$ $94^{\circ} \mathrm{C} 30 \mathrm{sec}, 56^{\circ} \mathrm{C} 40 \mathrm{sec}, 72^{\circ} \mathrm{C} 1 \mathrm{~min}$, close loop $72^{\circ} \mathrm{C} 5 \mathrm{~min}$. The DNA was diluted $1 / 100$. A total volume of $25 \mu \mathrm{L}$ mixtures composed of $13.45 \mu \mathrm{L}$ water, $2.5 \mu \mathrm{L} 10 \mathrm{x}$ reaction buffer (New England BioLabs, Ipswich, MA, USA), $0.2 \mu \mathrm{L} \mathrm{MgCl}_{2}$ (Bioline), $1.5 \mu \mathrm{L}$ dNTPs $150 \mu \mathrm{M}$ (Bioline, Luckenwalde, Germany), $0.75 \mu \mathrm{L}$ of each primer $0.3 \mu \mathrm{M}$ (Invitrogen by Thermo Fisher Scientific), $0.05 \mu \mathrm{L}$ of DNA polymerase $0.25 \mathrm{U}$ (New England BioLabs, Ipswich, MA, USA) and $6 \mu \mathrm{L}$ of DNA sample was used for PCR. A control PCR without DNA was set up to check for nonspecific amplification. The expected PCR products' size was 600-750 bp.

Lambda DNA was digested with the restriction enzyme PstI and used as a size standard. For agarose gel electrophoresis of the PCR products, $2 \mu \mathrm{L}$ blue juice were mixed with $2 \mu \mathrm{L}$ digested lambda DNA (25 ng/mL). The PCR products were checked on agarose gel electrophoresis and purified using isopropanol precipitation and sent for sequencing as described above.

\section{Phylogenetic analysis}

Similarity searches in the sequences of the sequenced ITS1, 5.8S ribosomal subunit and ITS2 regions were done using BLAST in NCBI database (http://blast.ncbi.nlm.nih.gov/Blast.cgi). 
Alignments and phylogenic analysis of the above mentioned sequences were performed and phylogenetic trees were constructed using the software MEGA.7 (Kumar et al., 2015).

\subsubsection{Statistical analysis}

Significance of data on radial mycelium growth according to growth temperature and mycelium weight according to $\mathrm{pH}$ of the fungi F18 and F20 was analyzed using the software IBM SPSS for Macintosh version 22.0 (2013). Analysis of Variance (ANOVA) and post-hoc Tukey test in a completely randomized design were performed for the comparison of means of dependent variables and two tailed $\mathrm{P}$ values were considered as statistically significant on the level 0.05 .

\subsection{Identification of enniatin-detoxifying bacterium}

The identification of enniatin-degrading bacterium was done initially according to morphological examination and some biochemical characterization. Morphological parameters investigated included colony characteristics such as size, form, elevation, margin/edges, color, pigment production, opacity, surface, consistency and odor. Moreover, the biochemical characteristics including Gram stain, catalase test (3\%, 15\%), starch hydrolysis, SIM (indole, motility and sulfide production), nutrient gelatin stab, methyl red, citrate test and urease test were investigated. The bacterial growth at different temperatures and in different media (nutrient broth containing $6.5 \% \mathrm{NaCl}$, sabouraud dextrose agar, nutrient broth, starch agar, pectine agar and casein agar) was tested. A final step involving the molecular characterization of the microorganism was carried out involving DNA extraction, PCR and sequencing of the $16 \mathrm{~S}$ rRNA genes.

\subsubsection{Morphological characterization and optimal growing temperature of the bacterium K4}

The bacterium K4 was streaked onto nutrient agar plates using T-streaking method and grown for 24 hours at $25^{\circ} \mathrm{C}$. The features of the colonies such as size, form, elevation, margin/edges, color, pigment production, opacity, surface, consistency and odor were recorded.

To evaluate the optimal growth temperature in liquid medium, bacterium $\mathrm{K} 4$ was grown in full media (FM) broth at $25^{\circ} \mathrm{C}$ for 24 hours. The OD600 of the culture was measured and 
adjusted to 0.6 . A volume of $500 \mu \mathrm{L}$ of bacteria suspension was inoculated in $5 \mathrm{~mL}$ of tryptic soy broth and the tubes were incubated at $25^{\circ} \mathrm{C}, 28^{\circ} \mathrm{C}, 30^{\circ} \mathrm{C}, 35^{\circ} \mathrm{C}$ and $37^{\circ} \mathrm{C}$ respectively during a period of 24 hours. Each experiment was performed in 3 repetitions. After 24 hours, the bacterial cultures were diluted $1 / 2$ and the OD600 was measured.

A light smear of the bacterium $\mathrm{K} 4$ from a young isolated colony was inoculated onto nutrient agar containing $6.5 \% \mathrm{NaCl}$, nutrient broth, sabouraud dextrose agar, starch agar, pectin agar and casein agar. After 24 hours incubation at $30^{\circ} \mathrm{C}$, growth was recorded.

\subsubsection{Biochemical characterization of the bacterium $\mathrm{K} 4$}

\section{Gram Stain}

Gram Stain was performed according to Hucker's Modification of Gram's Stain method as described by Gephardt et al. (1981). Reagent crystal violet, Gram's Iodine, decolorizing agent and the counterstain were prepared as described by Gephardt et al. (1981).

\section{Catalase test}

The slide or drop catalase test was performed to detect the catalase production and activity according to Duke and Jarvis (1972), MacFaddin (2000) and Forbes et al. (2007).

\section{Starch Hydrolysis}

The starch agar media used were prepared according to Zimbro et al. (2009) and the method used for the starch hydrolysis test was the method described by Collin et al. (1995).

\section{Sulfide Indole Motility (SIM) test}

SIM test was performed using the SIM media (Difco, 1998; Green et al., 1951; Hiss, 1920). Motility test was carried out as described by Sulkin and Willett (1940), Difco (1998) and Green et al. (1951). Sulfur reduction test (Sulkin and Willett, 1940; Difco, 1998) and the indole test were performed according to Baron et al. (1990), Harley (2005) and MacFaddin (2000).

\section{Nutrient gelatin stab}

Gelatin stab test was performed as described by Difco Laboratories (2009). 


\section{Citrate test}

The procedure used was as described by Difco (1998), MacFaddin (2000), Harley (2005) and Reddy (2007). The Simmons citrate medium was prepared as described by Difco \& BBL Manual (2009).

\section{Urease test}

Urease test was conducted according to the Christensen's Method (Christensen, 1946).

\subsubsection{Molecular identification of bacterial isolate}

16S rRNA gene sequencing was performed for the molecular characterization of the active bacterium. The procedure encompasses three major steps: DNA extraction, PCR and sequencing. Then the phylogenic analysis was carried out.

\section{DNA extraction}

DNA extraction from enniatin detoxifying bacterium $\mathrm{K} 4$ was achieved according to a modified method of Ausubel et al., (1987). $1.8 \mathrm{~mL}$ of 24 hours bacterial culture on full media (FM) broth was put into a $2 \mathrm{~mL}$ sterile tube and centrifuged during 10 minutes at $20^{\circ} \mathrm{C}$ and 14000 RPM. The supernatant was carefully drawn off, the pellet was resuspended in $576 \mu \mathrm{L}$ TE-buffer and $30 \mu \mathrm{L}$ of $10 \%$ SDS were added followed by $3 \mu \mathrm{L}$ proteinase $\mathrm{K}(20 \mathrm{mg} / \mathrm{mL})$, the mixtures were incubated for 1 hour in a $37^{\circ} \mathrm{C}$ preheated water bath. $100 \mu \mathrm{L} \mathrm{NaCl} 5 \mathrm{M}$ and $80 \mu \mathrm{L} 10 \% \mathrm{CTAB}$ (in $1 \times \mathrm{TE}, 0.7 \mathrm{M} \mathrm{NaCl}$ ) were respectively added to the mixture and incubated in a $65^{\circ} \mathrm{C}$ preheated water bath for 10 minutes. After this incubation, $700 \mu \mathrm{L}$ of chloroform/isoamyl alcohol 24:1 (v/v) were added and the whole sample was thoroughly mixed and kept on ice for 10 minutes. The tubes were then centrifuged at 14000 RPM during 10 minutes in a $4^{\circ} \mathrm{C}$ precooled centrifuge. The aqueous viscous phase was recovered, and $30 \%$ PEG was added to a final concentration of $6.5 \%$ (divide volume of the aqueous phase by 3.6). The content of the tubes was cautiously mixed by tilting and left for precipitation for 20 30 minutes at room temperature. The samples were centrifuged for 10 minutes at $4^{\circ} \mathrm{C}$ and 14000 RPM. After centrifugation, the supernatant was discarded and the pellet washed twice with cold $\mathrm{EtOH}(70 \%)$ with the precaution not to loose the pellet. After the second washing, the rest of EtOH was spinned down and removed as much as possible using a pipette. The pellet was dried at $30^{\circ} \mathrm{C}$ in the SpeedVac (Eppendorf concentrator 5301, $1400 \mathrm{RPM}$ ) for 10 minutes and resuspended in $50 \mu \mathrm{L}$ of TE-buffer overnight. 


\section{Electrophoresis}

To check for the success of the DNA extraction step, $2 \mu \mathrm{L}$ DNA solution, $1 \mu \mathrm{L}$ and $2 \mu \mathrm{L}$ of the standard Lambda-DNA (25 $\mathrm{ng} / \mathrm{mL}$ Lambda DNA) were run on $0.8 \%$ agarose gel as described above.

\section{PCR}

Molecular identification of the bacterial isolate was achieved through the amplification partial sequence $16 \mathrm{~S}$ rRNA genes using the bacterial universal primers 27F (5'AGAGTTTGATCMTGGCTCAG) (Lane, 1991) and bacterial primer 1100R (5'AGGGTTGCGCTCGTTG) (Turner et al., 1999) with cycling conditions of $94^{\circ} \mathrm{C} 30 \mathrm{sec}, 10 \times$ $\left(94^{\circ} \mathrm{C} 30 \mathrm{sec} ; 62^{\circ} \mathrm{C}-53^{\circ} \mathrm{C}-1^{\circ} \mathrm{C} /\right.$ cycle $\left.40 \mathrm{~min} ; 72^{\circ} \mathrm{C} 1 \mathrm{~min}\right)$, close loop $30 \times\left(30 \mathrm{x} 94^{\circ} \mathrm{C} 30 \mathrm{sec}\right.$; $56^{\circ} \mathrm{C} 40 \mathrm{~min} ; 72^{\circ} \mathrm{C} 1 \mathrm{~min}$ ), close loop $72^{\circ} \mathrm{C}$ 5minutes. The DNA was diluted $1 / 100$ and $1 / 1000$. PCR was carried out with a total volume of $25 \mu \mathrm{L}$ composed of $18.45 \mu \mathrm{L}$ water, $2.5 \mu \mathrm{L} 10 \mathrm{x}$ reaction buffer (New England BioLabs, Ipswich, MA, USA), $1.5 \mu \mathrm{L}$ dNTPs $150 \mu \mathrm{M}$ (Bioline, Luckenwalde, Germany), $0.75 \mu \mathrm{L}$ of each primer $0.3 \mu \mathrm{M}$ (Invitrogen by Thermo Fisher Scientific), $0.05 \mu \mathrm{L}$ of Taq DNA polymerase $0.25 \mathrm{U}$ (New England BioLabs, Ipswich, MA, USA and $1 \mu \mathrm{L}$ of DNA sample. A control PCR without DNA was set up to check for nonspecific amplification. The expected PCR products' size was $1000 \mathrm{bp}$.

A marker of $1000 \mathrm{bp}$ (Forever $100 \mathrm{bp}$ Ladder Personalizer, Seegene) was used as a size standard. $2 \mu \mathrm{L}$ blue juice were mixed with $2 \mu \mathrm{L}$ marker $(25 \mathrm{ng} / \mathrm{mL})$. The PCR product was checked on a gel, prepared for sequencing and sequenced as described above.

\section{Phylogenetic analysis}

Similarity searches in the sequences of the sequenced 16S rRNA gene, alignments and phylogenic analysis were performed as described above.

\subsubsection{Statistical analysis}

Significance of OD600 data related to growth of bacterium K4 at different temperatures was analyzed using the software IBM SPSS for Macintosh version 22.0 (2013). Analysis of Variance (ANOVA) and post-hoc Tukey test in a completely randomized design were performed for the comparison of means of dependent variables, two tailed $\mathrm{P}$ values were considered as statistically significant on the level 0.05 . 


\section{Results}

\subsection{Identification of enniatin-detoxifying fungi}

\subsubsection{Optimal growth temperature and $\mathbf{p H}$}

In general, the growth of fungus F18 was slower compared to fungus F20 when grown on PDA (growth expressed as radial growth) or on PDB (growth expressed as dried mycelium weight) at various tested temperatures and $\mathrm{pH}$ values.

The fungus F20 grew slower at temperatures lower than $20^{\circ} \mathrm{C}$ and faster at temperatures above $24^{\circ} \mathrm{C}$. The fastest growth of fungus $\mathrm{F} 20$ was recorded at $28^{\circ} \mathrm{C}$, where the radial growth reached about $40 \mathrm{~mm}$ after 10 days and was significantly different $(\mathrm{P}<0.05)$ from other values, while the lowest growth was observed when the fungus was incubated at $12^{\circ} \mathrm{C}$ (Figure 1, A). For fungus F20, the optimal $\mathrm{pH}$ growth in PDB was between 3.6 and 8 where the values of the dried mycelium reached about $0.25 \mathrm{~g}$, while the lowest growth was observed in PDB pH 2.4 and a moderate growth at pH 3 (Figure 2, A).

When the fungus F18 was grown on PDA, a maximum radial growth of about $16 \mathrm{~mm}$ at $24^{\circ} \mathrm{C}$ was recorded after 10 days. The lowest radial growth was recorded when the fungi was incubated at $32^{\circ} \mathrm{C}$ (almost no growth) and $12^{\circ} \mathrm{C}$ (about $6 \mathrm{~mm}$ ) (Figure 1, B). No significant difference $(\mathrm{P}>0.05)$ between the values of the dried mycelium weight of fungus F18 was observed among the tested $\mathrm{pH}$ values. According to the results obtained, the fungus F18 can tolerate a wide range of $\mathrm{pH}$ in liquid media such as $\mathrm{PDB}$. However, the optimal $\mathrm{pH}$ growth in PDB was between 3 and 3.6, where the dried mycelium weight reached about $0.2 \mathrm{~g}$, while the lowest growth was observed at higher $\mathrm{pH}$ values from 5.6 to 8 in PDB (Figure 2, B). 

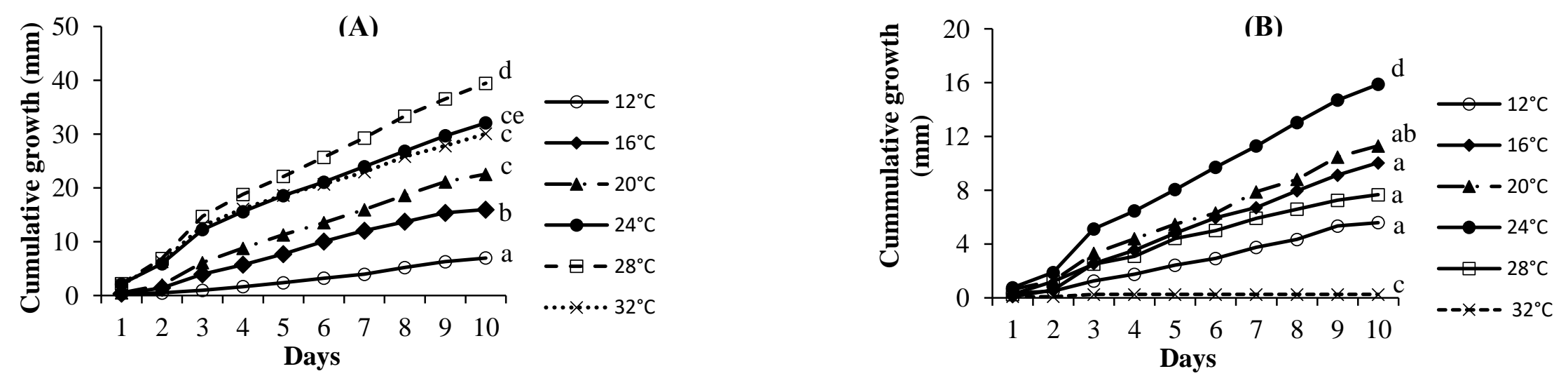

Figure 1: Mean values of the cumulative radial growth of fungi F20 (A) and F18 (B) grown on PDA plates at different temperatures.

(A), (B): $a, b, c, d$, e Lines (mean \pm Standard deviation) carrying the same letter represent values (after 10 days) statistically not different $P>0.05$, $n=3$.
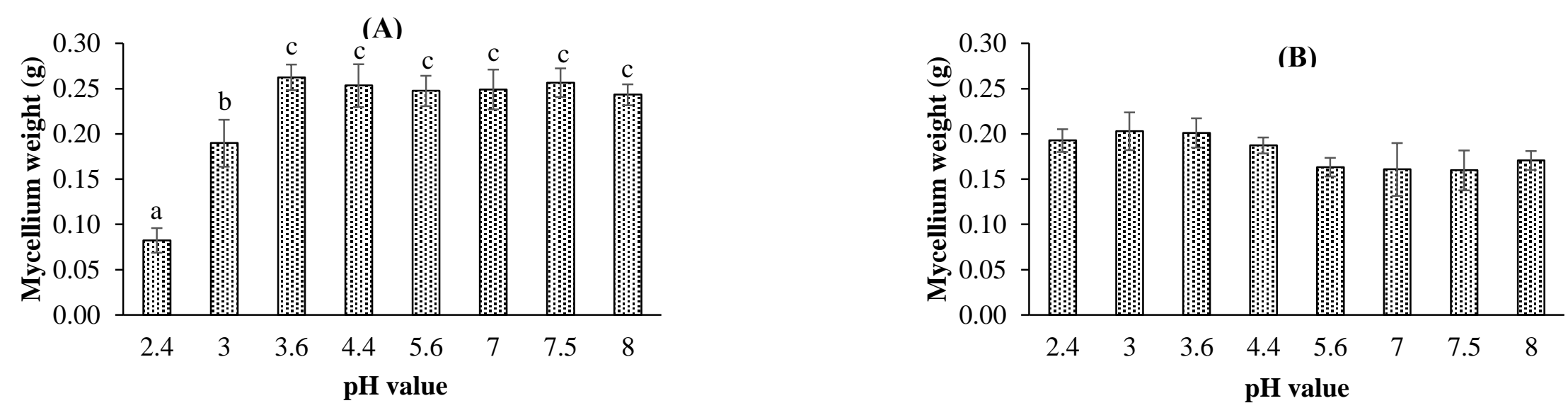

Figure 2: Mean values of the dried mycelium weight of fungi F20 (A) and F18 (B) after culture in PDB with different pH values for 10 days at $28^{\circ} \mathrm{C}$ for $\mathrm{F} 20$ and $24^{\circ} \mathrm{C}$ for $\mathrm{F} 18$. (A): a, b, c Histograms (mean \pm Standard deviation) carrying the same letter represent values statistically not different $P>0.05$. (B): No statistically difference among the values $P>0.05, n=4$. 


\subsubsection{Morphological characterization of fungal isolates}

\section{Visual description of the colony characteristics}

Morphological characteristics of both fungi F18 and F20 are recorded in Table 1. The colonies of fungus F20 were circular with irregular margin. The mycelium color was white on the top and cream on the reverse plate. When observed from the side, colonies presented abundant air mycelium. For fungus F18, the colonies were flat and slightly raised in the center. On the surface of the agar, the colonies were membrane-like and embedded in the agar (forming few lines from the center to the margin of the colony). The colonies were white in color, while the reverse plate was yellowish to orange in color. Fungi F20 and F18 grown on PDA for 14 days at $25^{\circ} \mathrm{C}$ are shown in the Figure 3.

Table 1: Description of the colony characteristics of fungal strains F18 and F20 after growth for 7 days on PDA at $24^{\circ} \mathrm{C}$ and $28^{\circ} \mathrm{C}$ for $\mathrm{F} 18$ and $\mathrm{F} 20$ respectively.

\begin{tabular}{lcc}
\hline Parameters & F 18 & F20 \\
\hline Culture medium, age, growth temperature & PDA, 7 days, $24^{\circ} \mathrm{C}$ & PDA, 7 days, $28^{\circ} \mathrm{C}$ \\
Growth rate* & 1.61 & 4.18 \\
Colony margin and structure & Circular and regular & Circular and irregular \\
Colony surface and color on agar surface & Smooth, orange & Smooth, white-cream \\
Colony color from the top & White & Cream \\
Colony color from underneath & Orange & Cream \\
Colony appearance from the side & Little aerial hyphae & Abundant aerial hyphae \\
Excretion into the media & No & No \\
Oil droplets and aerial mycelium floccose** & No & Yes \\
\hline
\end{tabular}

* Radial colony expansion in $\mathrm{mm} /$ day during 7 days

** After 14 culture days 

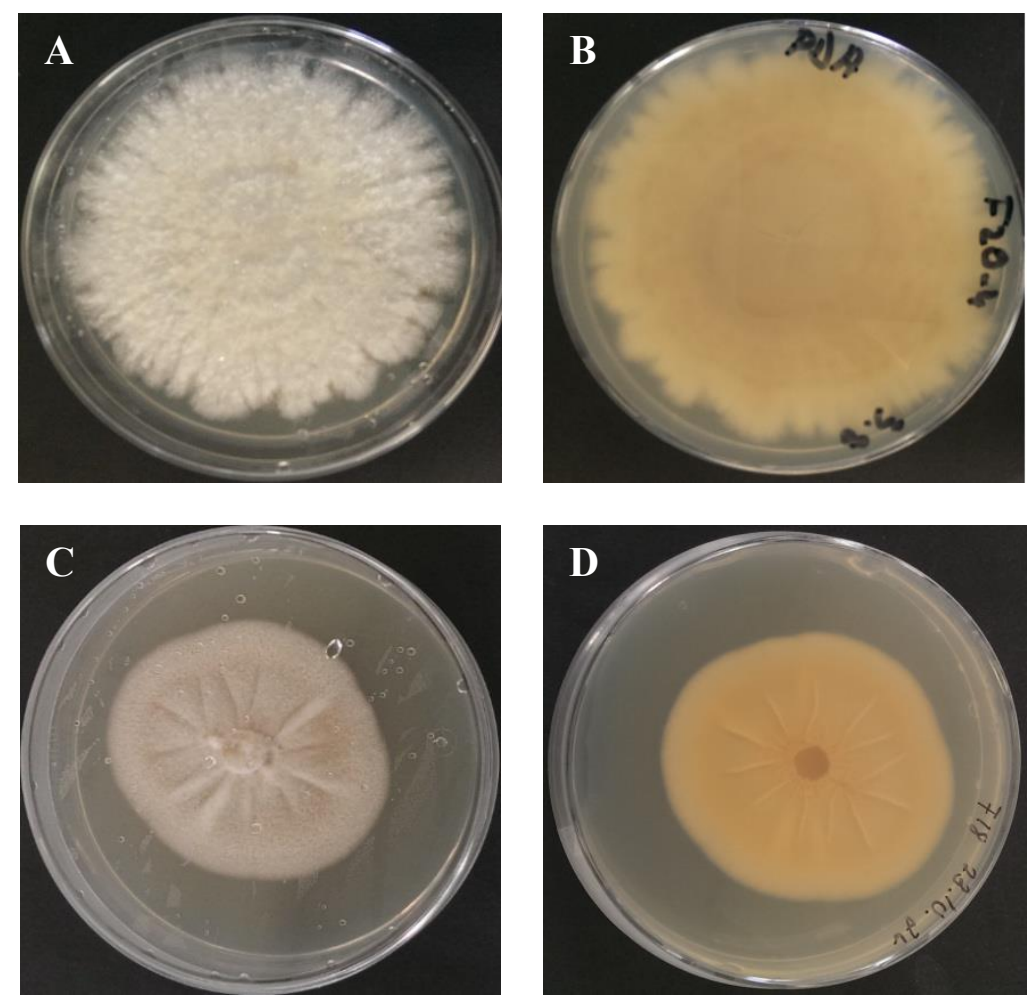

Figure 3: Colony of fungi F20 (A: top view; B: reverse plate view) and F18 (C: top view; D: reverse plate view) grown on PDA for 14 days at $25^{\circ} \mathrm{C}$.

\section{Microscopic description of the fungal isolates}

\section{Fungus F20}

Morphological features of fungus F20 under light microscope (100x) are shown in Figure 4. The conidia (Figure 4-A \& B) of the fungus F20 had variable sizes corresponding to different developmental stages and possess an oblong shape. The sizes are as follows: length: $5.2 \mu \mathrm{m}$ and width: $2.2 \mu \mathrm{m}$ for microconidia; length: $11.63 \mu \mathrm{m}$ and width: $3.7 \mu \mathrm{m}$ for macroconidia. Conidia were produced singly and presented pigmentation but no septae. The conidiophores (Figure 4-C \& D) were long and frequently branched. The phialides (Figure 4-C \& D) were medium sized, elongate-ampulliform in shape and possessed a basal septum. Moreover, the hyphae were pigmented and septate (Figure 4-E \& F). Branchings of hyphae to the conidiophores were regular. Clamp connections and chlamydospores were also observed. 

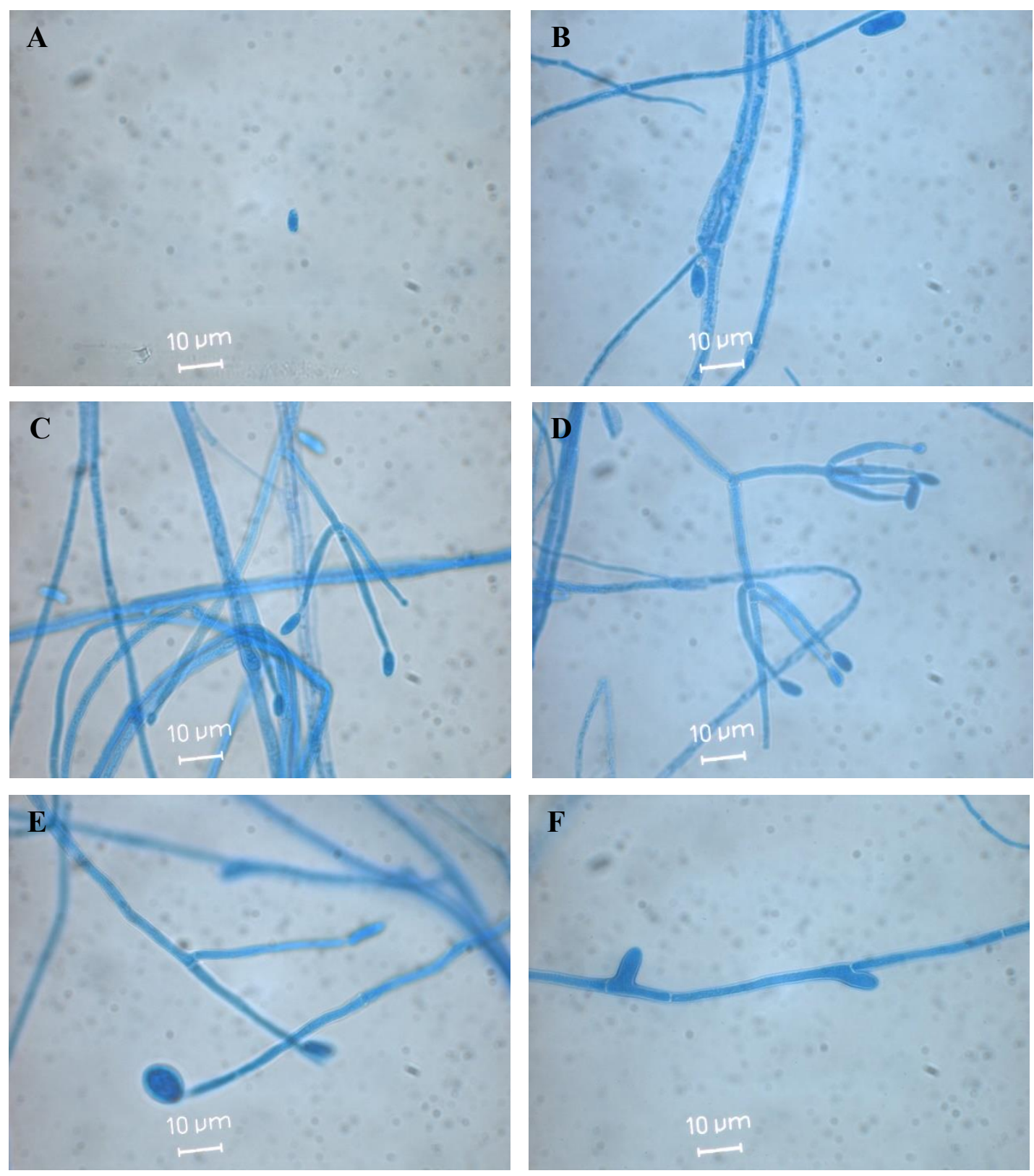

Figure 4: Microscopic structures of fungus F20 under light microscope (100x). (A) Conidia, (B) Macroconidia \& hyphae, (C) \& (D) Conidiophores, phialides, conidia \& hyphae, (E) Chlamydospore \& hyphae, (F) Clamps connection \& hyphae.

\section{Fungus F18}

Morphological features of fungus F18 under light microscope (100x) are shown in Figure 5. The conidia (Figure 5-A \& B) were fast developing during microscopic observation and have an oblong-elliptical shape. Their sizes were variable, length: $1.4 \mu \mathrm{m}$ and width $1.1 \mu \mathrm{m}$ for microconidia, length: $2.6 \mu \mathrm{m}-4.5 \mu \mathrm{m}$ and width: $1.1 \mu \mathrm{m}$ for intermediate conidia; length: 6.0 $\mu \mathrm{m}$ and width $1.6 \mu \mathrm{m}$ for macroconidia. Conidia were pigmented and aseptate. Fungus F18 
showed individual phialides that were long sized, elongated-ampulliform in shape; they were regularly branched to the hyphae and possessed a basal septae (Figure 5-C \& D). Hyphae (Figure 5-E \& F) of fungus F18 were pigmented and aseptate.
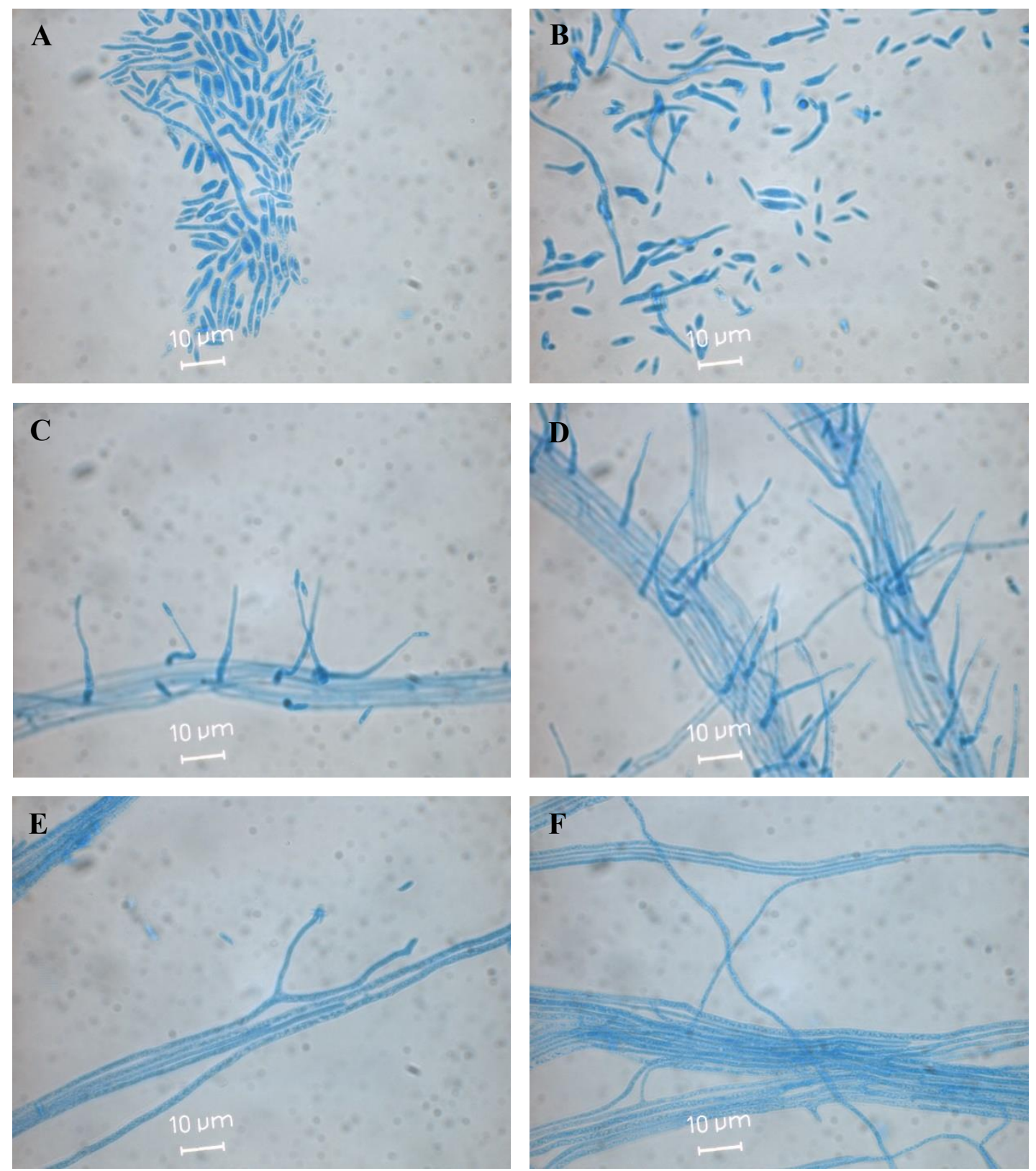

Figure 5: Microscopic structures of fungus F18 under light microscope (100x), (A) and (B) Micro- and macroconidia, (C) and (D) Conidiophores, phialides and hyphae, (E) and (F) Hyphae. 


\subsubsection{Molecular characterization of fungal isolates}

\section{Gel electrophoresis of extracted DNA and PCR products}

The agarose gel electrophoresis of total DNA extracted of fungi F18 and F20 is shown in Figure 6-A. With comparison of the sample to the lambda DNA (slot 1), a successful DNA extraction was achieved. The PCR amplification of the extracted DNA using primers EF1, EF2, ITS1 and ITS4 was successful as shown in Figure 6-B and as expected, fragments having about 600-750 base pairs were generated according to comparison with the molecular marker used (Lambda DNA digested with PstI). The products are indicated using an arrow in Figure 6-B.

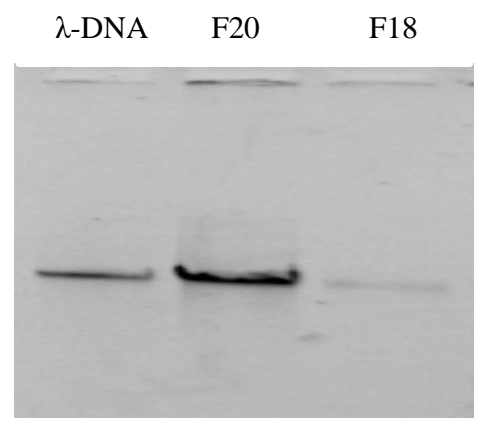

A

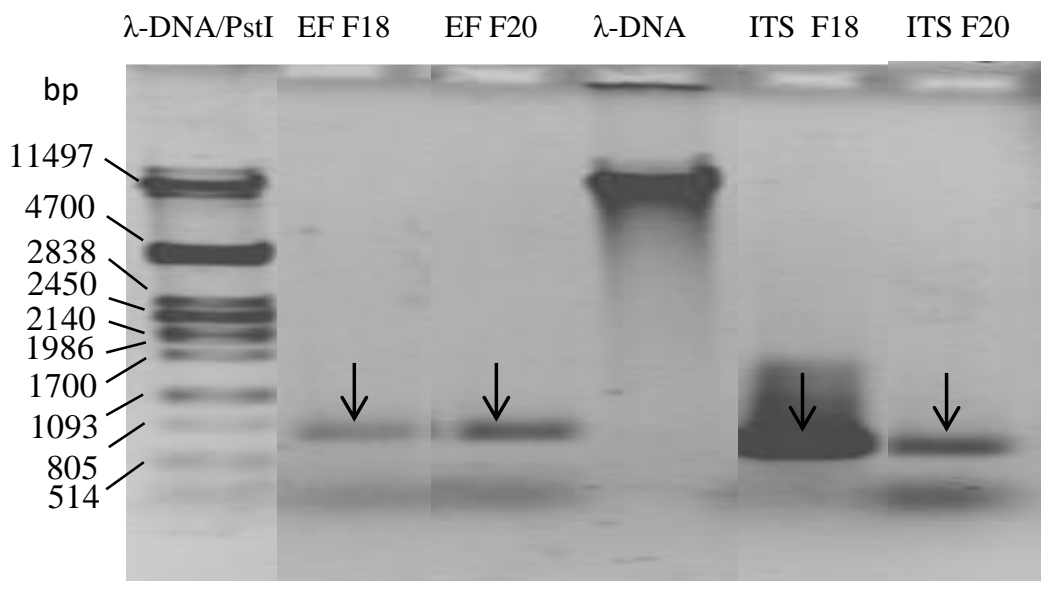

B

Figure 6: A: Gel electrophoresis of DNA extraction (from left to right: Lambda DNA, extracted DNA of fungus F20, extracted DNA of fungus F18); B: Gel electrophoresis of PCR products of EF1 \& 2 and ITS $1 \& 4$ (from left to right: molecular marker digested with PstI, PCR products using EF1 and EF2 for fungus F18, PCR products using EF1 and EF2 primers for fungus F20, Lambda DNA, PCR products using ITS1 and ITS4 primers for fungus F18 and fungus F20).

\section{Phylogenetic analysis of fungi F20 and F18}

Although the PCR amplification and DNA sequencing using the elongation factors 1 and 2 primers were successful, the blast of the sequenced regions did not give conclusive results concerning the molecular taxonomy of the microorganisms. Since a highly mutated area makes species classification possible, probably in this case there was not enough variation in the region of the DNA to distinguish the two species. So the sequence of the translation 
elongation factor 1-alpha (TEF-1 $\alpha$ ) gene was not suitable for the molecular identification of the two fungal species in this study.

The phylogenetic tree obtained using primers pairs ITS1 and ITS4 (including ITS1 and ITS2 regions, and 5.8S ribosomal RNA sequences of the DNA) of fungal strain F20 and Clonostachys species is shown in Figure 7. The fungal strain F20 revealed 100\% similarity with several C. rosea strains (BBA68698, R2-4, 10066) and Bionectria ochroleuca SBBEB. C. rosea was formerly known as Gliocladium roseum, teleomorph: Bionectria ochroleuca (Schroers et al., 1999).

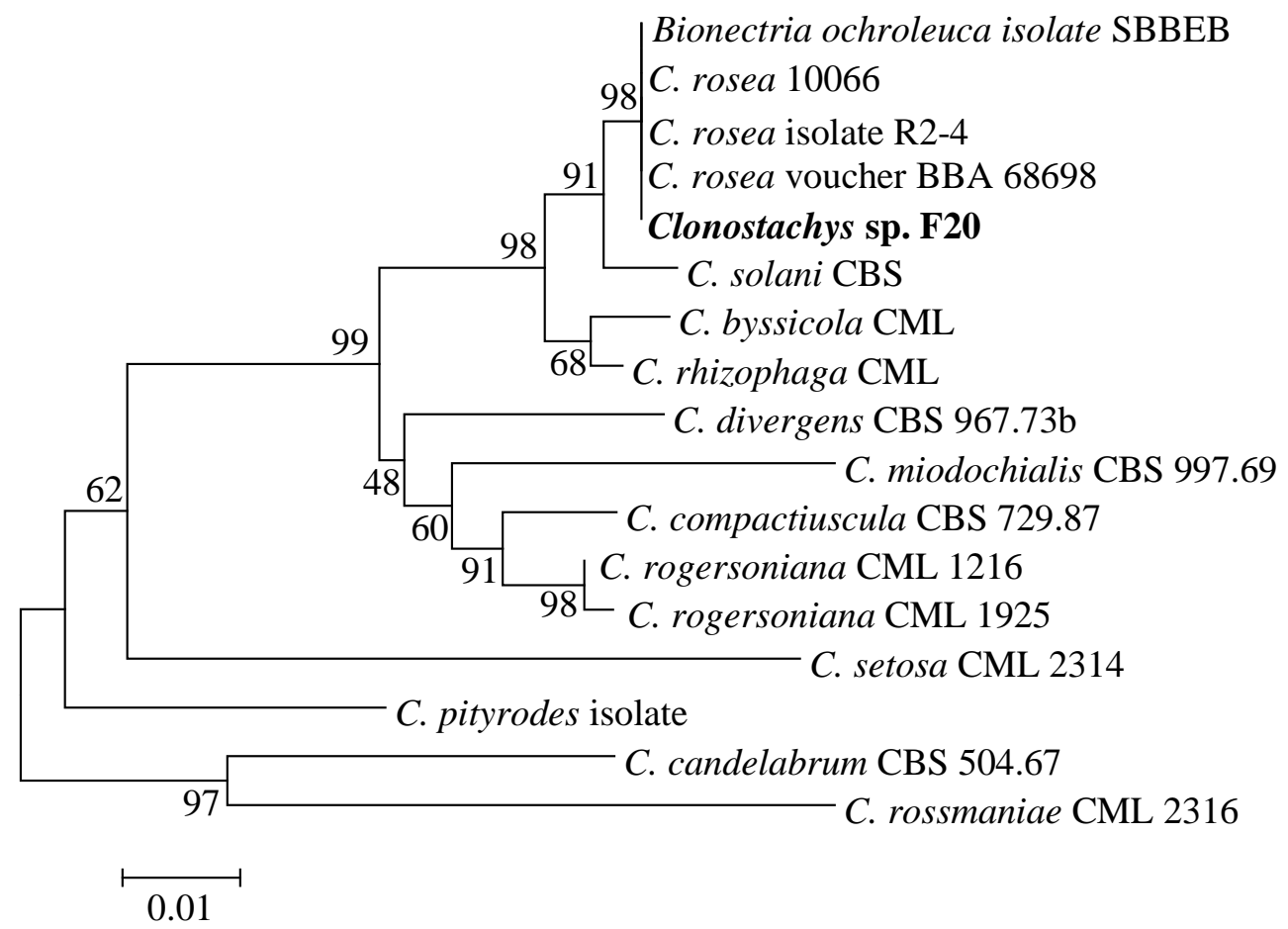

Figure 7: Neighbor-joining phylogenic tree based on the DNA sequences using ITS1 and ITS4 primers of fungus F20 and related fungi genera. At major nodes, bootstrap percentages for 1000 resamplings are shown. The scale bar represents 0.01 nucleotide substitutions per nucleotide position.

Clonostachys species include: Bionectria ochroleuca isolate SBBEB (GenBank accession number: EU484311.1), C. rosea 10066 (KC819614.1), C. rosea isolate R2-4 (KT876552.1), C. rosea voucher BBA 68698 (KT215192.1), C. solani strain CBS 228.74 (AF358243.1), C. byssicola strain CML 2404 (KC806271.1), C. rhizophaga strain CML 2312 (KC806275.1), C. divergens strain CBS 967.73b (AF210677.1), $C$. miodochialis strain CBS 997.69 (AF210674.1), C. compactiuscula strain CBS 729.87 (AF358242.1), C. rogersoniana strain CML 1216 (KC806287.1), C. rogersoniana strain CML 1925 (KC806292.1), C. setosa strain CML 2314 (KC806294.1), C. pityrodes isolate C40376SNA1CC1089 (JQ411387.1), C. candelabrum strain CBS 504.67 (AF210668.1), C. rossmaniae strain CML 2316 (KC806299.1).

According to the results of phenotypic and microscopic observations and the phylogenetic analysis based on the DNA sequences using ITS1 and ITS4 primers, the fungal strain F20 
showed 100\% similarities with strains Bionectria ochroleuca (former name of C. rosea), C. rosea 10066, C. rosea $\mathrm{R} 2-4$, C. rosea BBA 68698 .

Figure 8 below shows results of the phylogenetic analysis of DNA sequences of the 5.8S ribosomal RNA subunit and the ITS1 and ITS2 regions. Sequence comparison with other fungi of the same species showed high sequence identity. Fungal strains S. strictum NRRL 47828, NRRL 47825, T66M were grouped together with the fungus F18, therefore the fungus F18 was identified as Sarocladium strictum commonly known as Acremonium strictum.

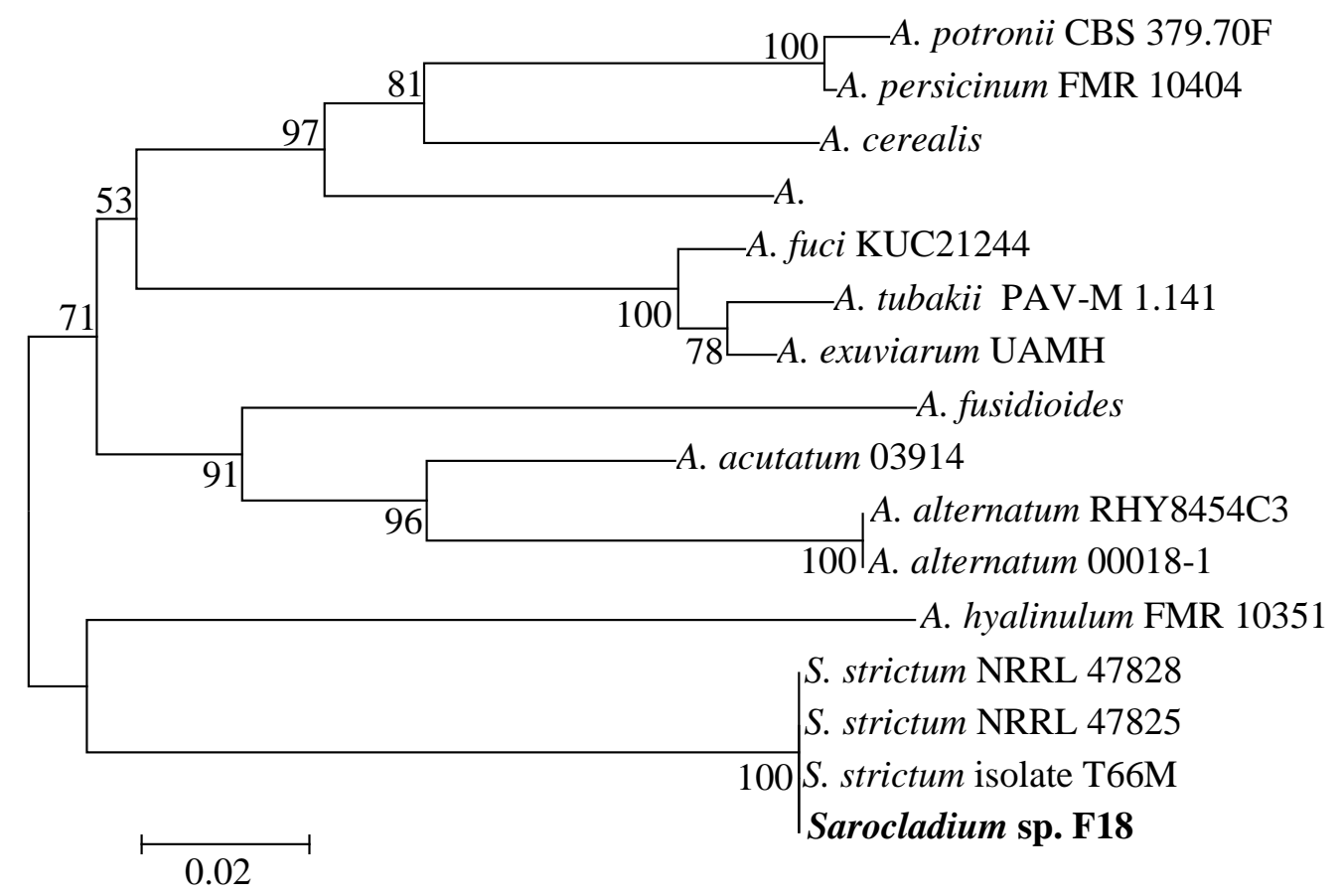

Figure 8: Neighbor-joining phylogenic tree based on the DNA sequences using ITS1 and ITS4 primers of fungus F18 and related fungi genera. At major nodes, bootstrap percentages for 1000 resamplings are shown. The scale bar represents 0.01 nucleotide substitutions per nucleotide position.

Acremonium species include: A. potronii CBS 379.70 (GenBank accession number: AY632655.1), A. persicinum FMR 10404 (F KP131529.1), A. cerealis HSAUP063188 (FJ914717.1), A. cyanophagus (DQ393594.1), A. fuci KUC21244 (KT207762.1) , A. tubakii isolate PAV-M 1.141 (KF915990.1), A. exuviarum UAMH 9995 (AY882946.1), A. fusidioides (FN706544.1), A. acutatum 03914 (KT878339.1), A. alternatum RHY8454C3 (KM268869.1), A. alternatum 00018-1 (KT192193.1), A. hyalinulum FMR 10351 (NR 131321.1), S. strictum NRRL 47828 (GU183127.1), S. strictum NRRL 47825 (GU183126.1), S. strictum isolate T66M (HM052811.1), Sarocladium sp. F18. 


\subsection{Identification of enniatin detoxifying bacterium}

\subsubsection{Morphological characterization and optimal growing temperature of the bacterium K4}

The colonies of bacterium K4 were rough with irregular margin; the overall evaluated characteristics are summarized in the Table 2 below. On nutrient agar, the colonies of bacterium K4 had a cream color (lighter as on FM agar). At the same temperature, growth of the studied bacterium was slower on nutrient agar than on FM agar. Figure 9 shows the bacterium $\mathrm{K} 4$ grown on nutrient agar and on FM agar.

Table 2: Phenotypic characteristics of the bacterium K4 observed on FM agar after 24 hours at $28^{\circ} \mathrm{C}$.

\begin{tabular}{lc}
\hline Colony characteristics & Bacterium K4 \\
\hline Size & Medium (variable) \\
Form & Irregular \\
Elevation & Flat \\
Margin/edge & Undulate \\
Color & Cream \\
Pigment production & No \\
Opacity & Opaque \\
Surface & Rough/ dull \\
Consistency & Buttery \\
Odor & Putrid \\
\hline
\end{tabular}
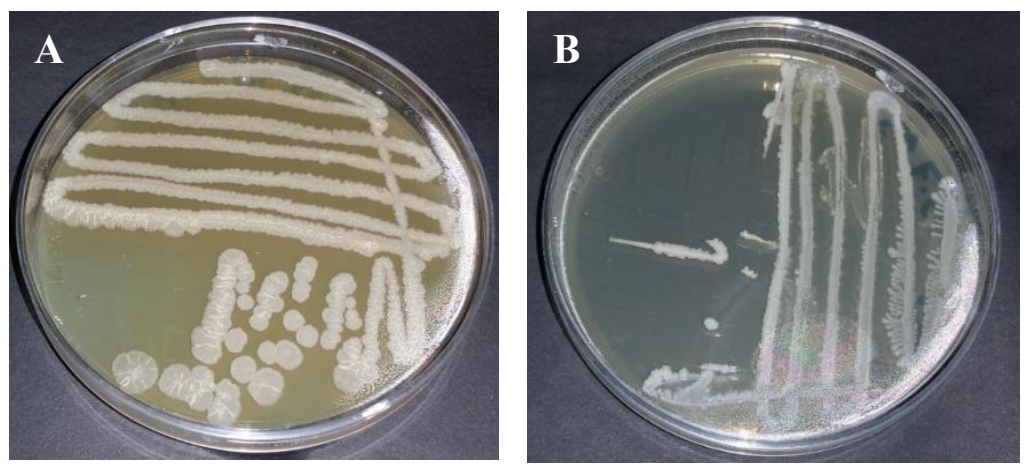

Figure 9: Bacterium K4 grown on FM agar pH 7.2 (A) and on nutrient agar (B) both for 24 hours at $28^{\circ} \mathrm{C}$. 
The optimal growth temperature of bacterium K4 in tryptic soy broth was between $28^{\circ} \mathrm{C}$ and $30^{\circ} \mathrm{C}$ (Figure 10). At those temperatures, the values of the OD600 were significantly different $(\mathrm{P}<0.05)$ from values obtained at 25,35 and $37^{\circ} \mathrm{C}$. The growth was expressed by the values of OD600 of $1 / 2$ diluted cultures after 24 hours culture. Bacterium K4 showed lowest growth at $25^{\circ} \mathrm{C}$, while its growth was moderate at $35^{\circ} \mathrm{C}$ in tryptic soy broth. In contrary to cultures at optimal growth temperatures, the cultures at $25^{\circ} \mathrm{C}$ or $37^{\circ} \mathrm{C}$ were clearer and thinner showing a low cell number.

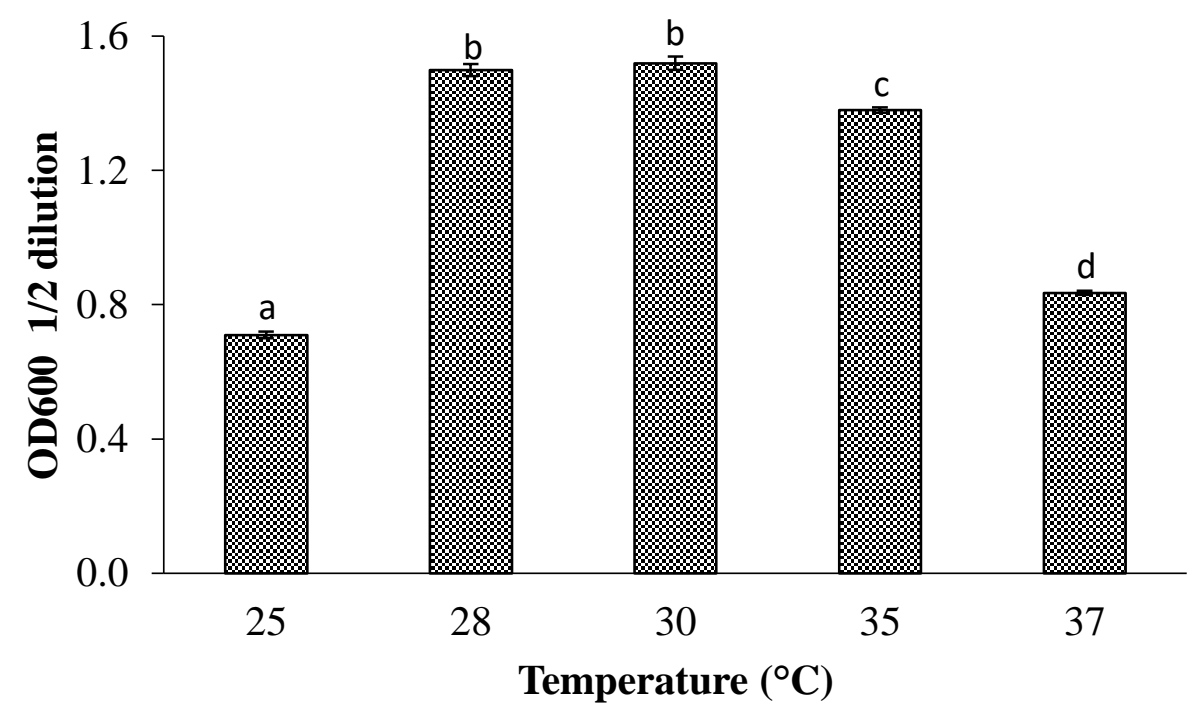

Figure 10: Optical density (OD600) of bacterium K4 grown in tryptic soy broth during 24 hours for the determination of optimal temperature growth of bacterium K4.

$a, b, c:$ Histograms (mean \pm Standard deviation) carrying the same letter represent values statistically not significant $P>0.05, n=3$.

\subsubsection{Biochemical characterization of the bacterium $\mathrm{K} 4$}

The physiological and biochemical characterization of the bacterium K4 (including Gram staining, starch hydrolysis, Sulfide-Indole-Motility, nutrient gelatin, citrate, urease and catalase tests, growth on different media) are summarized in Table 3. Bacterium K4 is a gram positive bacterium, the cells of bacterium K4 were rod-shaped (Length: $2.85 \pm 0.39 \mu \mathrm{m}$ and Width: $0.58 \pm 0.12 \mu \mathrm{m}$ ), arranged singly or in pairs (Figure 11). The features of K4 were consistent with the description of Bacillus sp. in Bergey's Manual of Systematic Bacteriology (Claus and Berkeley, 1986). 
Table 3: Biochemical characteristics of bacterium K4

\begin{tabular}{|c|c|c|}
\hline Biochemical tests & Bacterium K4 & \\
\hline Gram staining & + & \\
\hline Starch hydrolysis & + & $\therefore$ \\
\hline Sulfide & - & \\
\hline Indole & - & \\
\hline Motility & $+1-$ & $1=x$ \\
\hline Nutrient gelatin stab & - & \\
\hline Citrate & + & \\
\hline Urease & - & 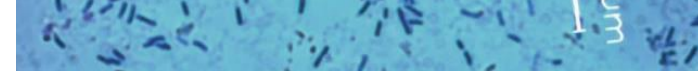 \\
\hline Catalase 3\% & + & ( \\
\hline Catalase $15 \%$ & + & Figure 11: Microscopic examination of \\
\hline Growth on & & bacterium K4, Rod-shaped cells \\
\hline $6.5 \% \mathrm{NaCl}$ & + & observed after Gram stain under light \\
\hline Sabouraud dextrose agar & + & microscope $(100 \times)$. \\
\hline Nutrient broth & + & \\
\hline Starch agar & + & \\
\hline Pectine agar & + & \\
\hline Casein agar & + & \\
\hline
\end{tabular}

\subsubsection{Molecular identification of bacterial isolate}

\section{Gel electrophoresis of extracted DNA and PCR product}

Figure 12-A shows the agarose gel electrophoresis of DNA extracted of bacterium K4. With comparison of the sample to the lambda DNA, a successful DNA extraction was achieved. The PCR amplification of the partial sequence of 16S rRNA gene resulted in a product of about $1000 \mathrm{bp}$ as expected (Figure 12-B). 


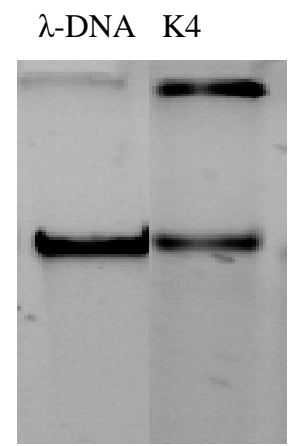

$\mathbf{A}$

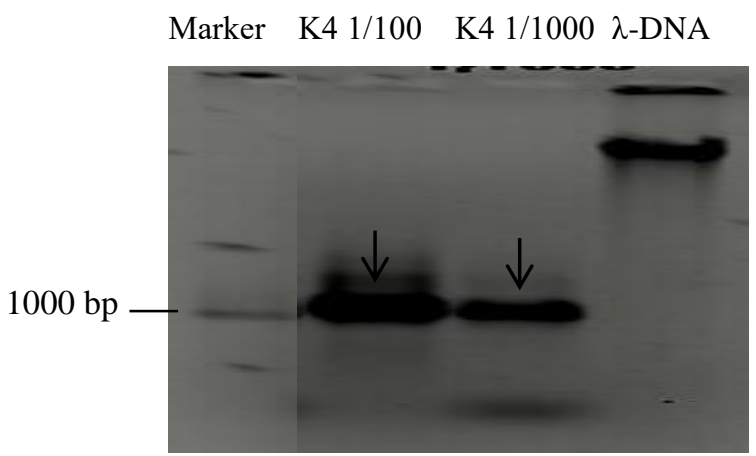

B

Figure 12: A: Gel electrophoresis of DNA extraction (from left to right: Lambda DNA, extracted DNA of bacterium K4); B: PCR products of the amplification of the 16S rRNA gene of bacterium K4 (from left to right: $1000 \mathrm{bp}$ marker, PCR product of the partial sequence of $16 \mathrm{~S}$ rRNA gene of bacterium K4 dilution 1/100; PCR product of the partial sequence of 16S rRNA gene of bacterium K4 dilution 1/1000; Lambda DNA).

\section{Phylogenetic analysis of bacterium K4}

The phylogenetic tree of the bacterium K4 and related bacillus species is shown in Figure 13, the bacterial strain K4 revealed 100\% identity with B. licheniformis strain CY-012, and very high similarity with B. licheniformis RPk and B. licheniformis SB 3131.

According to the results of phenotypic and microscopic observations, physiological and biochemical tests, and the phylogenetic analysis based on the partial sequence of the $16 \mathrm{~S}$ rRNA gene, the strain K4 was identified as B. licheniformis. 


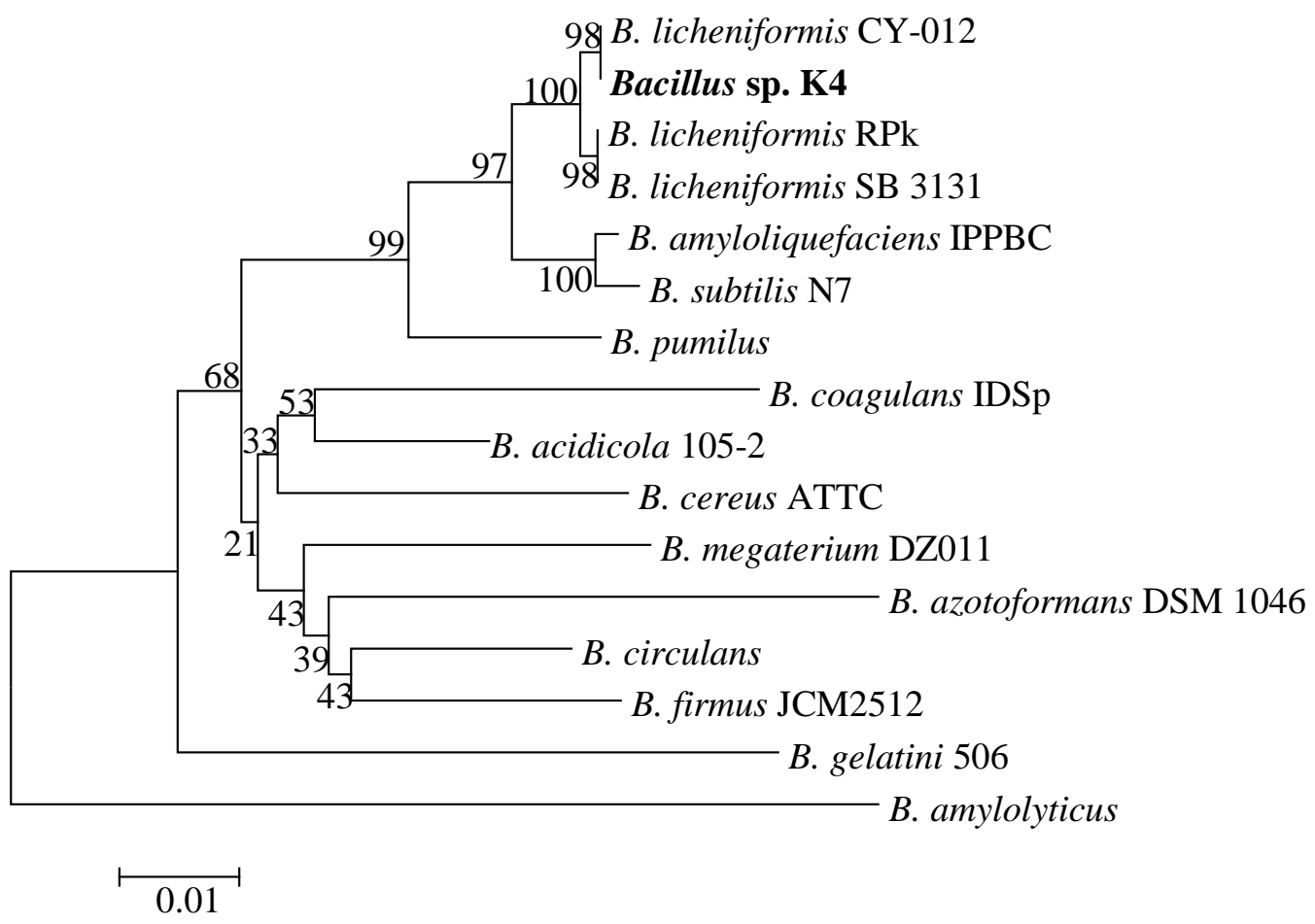

Figure 13: Neighbor-joining phylogenic tree based on the partial sequence of the 16S rRNA gene of bacterium K4 and related bacteria. At major nodes, bootstrap percentages for 1000 resamplings are shown. The scale bar represents 0.01 nucleotide substitutions per nucleotide position.

B. licheniformis CY-012 (GenBank accession number: KR349358.1), B. licheniformis RPk (EU445292.1) , B. licheniformis SB 3131 (GU191917.1), B. amyloliquefaciens IPPBC 10A (HQ2843041), B. subtilis N7 (JQ317780.1), B. pumilus ST277 (EU350371.1), B. cereus ATTC 14579 (NR_074540.1), B. coagulans IDSp (AF466695.1), B. acidicola 105-2 (NR_041942.1), B. megaterium DZ011 (QD408589.1), B. azotoformans DSM 1046 (NR 115576.1), B. circulans WZ12 (EF100968), B. firmus JCM2512 (NR_115581.1), B. gelatini 506 (DQ350818.1), B. amylolyticus (X60606.1). 


\section{Discussion}

This work was performed with the aim of identifying three microorganisms (one bacterium and two fungi) isolated as described in the previous chapter due to their property to degrade enniatins. The identification was made with the aid of morphological and molecular parameters of the microorganisms. Based on the results obtained during this study, the microorganisms capable of degrading enniatins previously labelled as K4, F20 and F18, it was revealed that K4, F20 and F18 were Bacillus licheniformis, Clonostachys rosea and Acremonium strictum respectively. The analysis of the translation elongation factor 1-alpha (TEF-1 $\alpha$ ) gene for the identification of both fungi did not give any conclusive results and therefore was not appropriate in this case. Since ribosomal genes are highly conserved, sequence variation between strains belonging to different species from distinct genera is less evident with rDNA gene sequence (Wayne et al., 1987).

\subsection{Bacterium K4: Bacillus licheniformis}

The 16S rDNA gene sequencing has allowed the identification of the bacterium K4 species. This technique is a great tool and by far the single most common molecular technique currently used for the purpose of bacterial species identification (Wilson, 1995). The technique of 16S rDNA gene sequencing for bacteria identification has been successfully used in several studies (Yi et al., 2011; Ikunaga et al., 2011; Tan et al., 2014). This technique relies on the sequence of the DNA that encodes for the 16S rRNA subunit and examines only a small portion of the microbial genome.

In the previous chapter, the ability of B. licheniformis to degrade enniatin A, A1 and B1 was demonstrated. This result joined an already published report on the effect of B. licheniformis in the reduction of the mycotoxin zearalenone (Yi et al., 2011). Although Yi et al. (2011) did not investigate the presence of degradation products, the adsorption of zearalenone by the bacterium cell wall was excluded due the absence of zearalenone in the B. licheniformis cell wall extract. Moreover, in a study carried out by Petchkongkaew et al. (2008), B. licheniformis was able to decrease the amount of aflatoxin B1 and ochratoxin A at a rate of $74 \%$ to $92.5 \%$ in liquid and solid media respectively. 
Regarding the use of microorganisms in food and feed, the character of not to produce harmful toxins is a prerequisite in order to avoid damaging health effects. In recent studies, the capacity of B. licheniformis strains CK1 and ATCC to produce enterotoxins was tested (Yi et al., 2011; Liu et al., 2013). It was found that both strains CK1 and ATCC are nonenterotoxin producing B. licheniformis. In addition, Liu et al. (2013) also showed that B. licheniformis CK1 is non-hemolytic and displayed high levels of extracellular xylanase, cellulase and proteinase activity.

Among the beneficial effects of $B$. licheniformis is the ability to produce proticin, a phosphorus-containing triene which has a strong activity against proteus bacteria (Präve et al., 1972, Katz and Demain, 1977), as well as against Mycobacterium tuberculosis (Callow et al., 1947).

According to the evidence of bio-transformation of enniatins A, A1 and B1 demonstrated in the previous chapter of this thesis, its potential use as a probiotic demonstrated previously and after further investigations concerning enterotoxins nonproduction by Bacillus licheniformis (in studies carried out by other authors), the possibility of applying bacterium K4 as a food and feed supplement for bio-detoxification of enniatins should be considered.

\subsection{Fungus F20: Clonostachys rosea}

Based on morphological features with comparison of those reported by Schroers (2001) and Toledo et al. (2006), the fungus F20 could belong to the genus Clonostachys. According to the results of phenotypic and microscopic observations and the phylogenetic analysis based on the DNA sequence using the ITS1 and ITS4 primers, the fungal strains F20 was identified as Clonostachys rosea.

Clonostachys rosea is a common soil fungus and mycoparasite (Schroers et al., 1999) from temperate and tropical regions (Schroers, 2001). The mycoparasitic effects of C. rosea have been demonstrated against several fungal plant pathogens such as Botrytis cinerea in strawberry (Cota et al., 2009), S. sclerotorium (Rodríguez et al., 2011) and F. graminearum (Kosawang et al., 2014). C. rosea has also been reported to be an entomopathogenic fungus of two leafhoppers pest namely Oncometopia tucumana and Sonesimia grossa (Toledo et al., 2006). 
The microbial activity of $C$. rosea on zearalenone (ZEN) was demonstrated several decades ago. El-Sharkawy and Abul-Hajj (1988) demonstrated an in vitro reduction of ZEN in 80-90\% yields by $G$. roseum (early name of $C$. rosea). The enzyme zearalenone lactonohydrolase catalyzing the reaction has been purified and cloned (Takahashi et al., 2002). Other authors demonstrated a ZEN dose-dependent induction of the zearalenone lactonohydrolase and suggested a specificity of the enzyme towards ZEN due to the absence of the induction enzyme expression by the known inducers of the Polyketide Synthase pathway (Kosawang et al., 2014). The degradation of ZEN by C. rosea as reported by Kakeya et al. (2002) involved the hydrolysis of the ester bond followed by a decarboxylation to produce 1-(3,5 dihydroxyphenyl)-10'-hydroxy-1'E-undecene-6'-one. The same authors demonstrated that the estrogenic activity of the degradation product did not show a potent estrogenic activity like that of ZEN and $17 \mathrm{~b}$-estradiol in the human breast cancer MCF-7 cell proliferation assay. Although $C$. rosea does not degrade fumonisins, it was shown that it suppresses the synthesis of fumonisnins by F. verticillioides in co cultures (Chatterjee et al., 2016).

The ability of $C$. rosea to degrade enniatins as described in our study highlights the importance of C. rosea for mitigation of toxins in foodstuffs.

\subsection{Fungus F18: Acremonium strictum}

Based on morphological features with comparison of those reported by Domsch et al., 2007, the fungus F18 could be classified as a member of the genus Acremonium. Further analysis of DNA sequences of 5.8S rRNA subunit, ITS 1 and ITS 2 regions followed by phylogenetic analysis helped to identify the fungal strain F18 as Acremonium strictum.

Acremonium strictum and other Acremonium species are not known to produce mycotoxins in food (Gams, 1971). A. strictum is a saprobe generally found in soil, plant debris, and rotting mushrooms (Guarro et al., 1997). Choi et al., (2008) reported the mycoparasitism of $A$. strictum against several fungi including Alternaria alternata, Bipolaris maydis, Magnaporthe grisea, Botrytis cinerea, Botrytis allii, Colletotrichum orbiculare, Fusarium oxysporum, Phytophthora capsici and Pythium ultimum. A previous study by Kim et al., (2002) attributed the mycoparasitic effect of $A$. strictum to verlamelin, an antifungal compound. This highlighted the possible use of $A$. strictum as a biological control agent for plant diseases caused by Botrytis cineria.

The production of cellulosic enzymes by Acremonium strictum was investigated by Goldbeck et al., (2013). CMCase, FPase, cellobiase and $\beta$-glucosidase activity were determined on 
several substrates including microcrystalline cellulose, carboxymethylcellulose and sugarcane bagasse. The results of the study suggested the use of $A$. strictum as a potential fungus for the production of new enzymes with potential biotechnological applications.

Acremostrictin has been isolated from liquid culture of $A$. strictum collected from choristida sponge off the coast of Korea. Acremostrictin exhibits a weak antibacterial activity against Micrococcus luteus, Salmonella typhimurium and Proteus vulgaris, as well as a moderate antioxidant activity leading to protection against oxidative stress induced cell death (Julianti et al., 2011).

In the scientific literature, few references are available regarding enniatin degrading bacteria. Nine bacteria of the gastrointestinal tract: $B b$. longum, $B b$. bifidum, Bb breve, Bb adolescents, Lb. rhamnosus, Lb. casei-casei, S. termofilus, Lb. ruminis, Lb. casei and 22 strains of $S$. cerevisiae were reported to significantly reduce enniatins in an in vitro fermentation system and three degradation products deriving from enniatin A, B and B1 were identified (Roig et al., 2013). The degradation of enniatins by six Bacillus subtilis strains (CECT 35, CECT 39, CECT 371, CECT 497, CECT 498, CECT 4522) was studied by Meca et al., (2014). All the tested strains were able to degrade enniatin A, A1, B and B1 in TSB. The mean reductions were $88.1 \% ; 83.1 \% ; 84.5 \%$ and $92.2 \%$ for enniatin $\mathrm{A}, \mathrm{A} 1, \mathrm{~B}$ and $\mathrm{B} 1$ respectively.

To the best of our knowledge and the thorough coverage of the available literature about the biological detoxification of enniatins, this is the first report of enniatin degradation by the microorganisms Bacillus licheniformis, Clonostachys rosea and Acremonium strictum. 


\section{Conclusion}

In conclusion:

- One enniatin-degrading bacterium (K4) and two enniatin-degrading fungi (F18 and F20) were phenotypically and genotypically characterized.

- Visual and microscopic observations enabled the genus identification of fungus F18 and fungus F20 as Acremonium and Clonostachys respectively.

- The amplification of the translation elongation factor 1-alpha (TEF-1 $\alpha$ ) gene sequence were not appropriate for the molecular identification of the two fungal species examined in this study.

- The sequence of 5.8S ribosomal subunit and the ITS1 and IT2 regions of the DNA enabled the identification of the fungi F18 and F20.

- Fungi F18 and F20 isolated respectively from wheat field soil (Göttingen) and hazel nut (Trader Joe's Haselnusskerne ganz, Märsch Importhandels-GmbH, Ulm) samples were identified as Acremonium strictum and Clonostachys rosea respectively.

- An initial characterization of the bacterium K4 using visual and microscopic observations, followed by some biochemical tests led to the classification of the bacterium K4 within the Bacillus genus.

- The analysis of the 16S rRNA gene sequence was required for the characterization of bacterium K4 species.

- The bacterium K4 isolated from a wheat field soil (Göttingen) was identified as Bacillus licheniformis. 


\section{Acknowledgements}

We acknowledge Dr. Athanassios Mavridis in the division of General Plant Pathology and Crop Protection of Georg-August-University Göttingen, for providing some bacteria strains used as positive or negative controls for the bacteria taxonomy tests. We thank Ruth Pilot for her involvement and collaboration during the molecular identification of enniatin detoxifying microorganisms.

\section{References}

Baron, E. and Finegold, S. (1990): Bailey and Scott's diagnostic microbiology, 8th ed., The Mosby Company, St. Louis, MO.

Boudergue, C., Burel, C., Dragacci, S., Favrot, M-C., Fremy, J-M. et al. (2009): Review of mycotoxin-detoxifying agents used as feed additives: mode of action, efficacy and feed/food safety, EFSA Supporting Publications, 6(9).

Brandfass, C. and Karlovsky, P. (2008): Upscaled CTAB-based DNA extraction and real-time PCR assays for Fusarium culmorum and Fusarium graminearum DNA in plant material with reduced sampling error, International Journal of Molecular Science, 9(11): 2306-2321.

Buchanan, R.E. and Gibbons, N.E. (1974): Bergey's manual of determinative bacteriology (Eighth edition), The Williams and Wilkins Co., Baltimore: 747-842.

Callow, R.K., Glover, R.E., D’Arcy Hart, P., Hills, G.M. (1947): Licheniformin, an antibiotic substance from Bacillus licheniformis, active against Mycobacterium tuberculosis, British Journal of Experimental Pathology, 28(6): 418-440.

Chatterjee, S., Kuang, Y., Splivallo, R., Chatterjee, P., and Karlovsky, P. (2016): Interactions among filamentous fungi Aspergillus niger, Fusarium verticillioides and Clonostachys rosea: fungal biomass, diversity of secreted metabolites and fumonisin production, BMC Microbiology, 16: 83.

Christensen, W.B., (1946): Urea decomposition as a means of differentiating Proteus and paracolon cultures from each other and from Salmonella and Shigella types, Journal of Bacteriology, 52:461-466. 
Claus, D. and Berkeley, R.C.W. (1986): Genus Bacillus Chon 1972. In Sneath PHA, Mair NS, Sharpe ME, Holt JG (eds) Bergey's manual of systematic bacteriology, vol 2. The Williams \& Wilkins Co., Baltimore: 1105-1139.

Collins, C.H., Lyne, P.M., Grange, J.M. (1995): Collins and Lyne's microbiological methods, Butterworth-Heinemann, Oxford, United Kingdom, $7^{\text {th }}$ ed, 117.

Cota, L.V., Maffia, L.A., Mizubuti, E.S.G. (2009): Biological control by Clonostachys rosea as a key component in the integrated management of strawberry gray mold, Biological Control, 50 (3): 222-230.

Difco \& BBL Manual (PDF) (2009): (2nd ed.). Sparks, Maryland: Difco Laboratories, Becton Dickinson and Company, 508. ISBN 0-9727207-1-5, Retrieved Jan 14, 2016.

Difco (1998): Difco manual, 11th ed. Difco Laboratories, Detroit, MI. Difco Laboratories (2009): Difco \& BBL manual: manual of microbiological culture media, 2nd ed,. Becton Dickinson and Company, Sparks, MD, 402-403.

Domsch, K.H., Gams, W., Anderson, T.-H. (2007): Compendium of soil fungi: 1-672.

Duke, P.B., Jarvis, J.D. (1972): The catalase test-a cautionary tale, Med Lab Technol, 29(2), 203-204.

Duvick, J., Rood, T. A. (2000): Beauvericin detoxification methods using bacteria, US 6126934 A.

El-Sharkawy, S., Abul-Hajj, Y.J. (1988): Microbial cleavage of zearalenone, Xenobiotica, 18(4): 365-371.

Forbes, B.A., Sahm, D.F., Weissfeld, A.S. (2007): Bailey and Scott's Diagnostic microbiology, 12th edition, Mosby Inc., St, Louis, ISBN: 9780323030656, Elsevier, 109-214.

Gams, W. (1971): Cephalosporium-artige Schimmelpilze (Hyphomycetes), G. Fischer, Stuttgart, 1-262.

Gephart, P., Murray, R.G. E., Costilow, R. N., Nester, E.W., Wood, W.A., Krieg, N.R., Phillips, G. B. (1981): Manual of Methods for General Bacteriology, ASM Press, Washington 
D.C. Gram. C.1884. Über die isolierte Färbung der Schizomyceten in Schnitt- und Trockenpräparaten, Fortschritte der Medizin, (2): 185-189.

Goldbeck, R., Ramos, M.M., Pereira, G.A., Maugeri-Filho, F. (2013): Cellulase production from a new strain Acremonium strictum isolated from the Brazilian Biome using different substrates, Bioresource Technology, 128: 797-803.

Green, R.A., Blum, E. F., DeCoro, C. T., Fairchild, R. B., Kaplan, M.T., Landau, J. T. and Sharp, T. S. (1951): Rapid methods for the detection of motility, Journal of Bacteriology, 62: 347.

Guarro, J., Gams, W., Puhhol, I., Gene, J. (1997): "Acremonium species: new emerging fungal opportunists-in vitro antifungal susceptibilities and review", Clinical Infectious Diseases, 25(5): 1222-1229.

Choi, G.J., Jin-Cheol, K., Kyoung, S.J., Kwang, Y.C., Heung, T.K. (2008): Mycoparasitism of Acremonium strictum BCP on Botrytis cinerea, the gray mold pathogen, Journal of Microbiololgy and Biotechnology, 18(1): 167-170

Harley, J. P. (2005): Laboratory exercises in microbiology, 6th ed. McGraw Hill, New York, NY.

Hiss, P. H. (1902): New and simple media for the differentiation of the colonies for typhoid, colon, and allied bacilli, Journal of Medical Research, 8:148-167.

Ikunaga, Y., Sato, I., Grond, S., NUmaziri, N., Yoshida, S., Yamaya, H. et al. (2011): Nocardioides sp. strain WSN05-2, isolated from a wheat field, degrades deoxynivalenol, producing the novel intermediate 3-epi-deoxynivalenol, Applied Microbiology, 89: 419-427.

Julianti, E., Oh, H., Jang, K.H., Lee, K.J., Lee, K.S., Oh, D.-C., Oh, K.-B. and Shin, J. (2011): Acremostrictin, a Highly Oxygenated Metabolite from the Marine Fungus Acremonium strictum, Journal of Natural Products, 74 (12): 2592-2594.

Kakeya, H., Takahashi-Ando, N., Kimura, M., Onose, R., Yamaguchi, I., Osada, H. (2002): Note Biotransformation of the Mycotoxin, Zearalenone, to a Non-estrogenic Compound by a 
Fungal Strain of Clonostachys sp., Bioscience Biotechnology Biochemnistry, 66 (12): 27232726.

Karlovsky, P. (1999): Biological Detoxification of Fungal Toxins and its Use in Plant Breeding, Feed and Food Production, Natural Toxins, 7: 1-23.

Katz, E. and Demain, A.L. (1977): Peptide antibiotics of Bacillus- chemistry, biogenesis and possible functions, Bacteriological Reviews, 41: 449-474.

Kim, J.-C., Choi, G. J., Kim, H.-J., Kim, H. T., Ahn, J. W. and Cho, K. Y. (2002): Verlamelin, an antifungal compound produced by a mycoparasite Acremonium strictum, Plant Pathology Journal, 18:102-105.

Klich, M.A. (2002): Identification of common Aspergillus species, Centraalbureau voor Schimmelcultures, Utrecht: The Netherlands.

Kosawang, C., Karlsson, M., Vélëz, H., Rasmussen, P.H., Collinge, D.B., Jensen, B., Jensen, D.F. (2014): Zearalenone detoxification by zearalenone hydrolase is important for the antagonistic ability of Clonostachys rosea against mycotoxigenic Fusarium graminearum, Fungal Biology, 118 (4): 364-373.

Kumar, S., Stecher, G. and Tamura, K. (2015): MEGA7: Molecular Evolutionary Genetics Analysis version 7.0, Molecular Biology and Evolution.

Lane, D.J. (1991): 16S/23S rRNA sequencing. In: Nucleic acid techniques in bacterial systematics. Stackebrandt, E. and Goodfellow, M., eds., John Wiley and Sons, New York, NY: 115-175.

Lennette, E.H., Balows, A., Hausler, W.J., Shadomy, H.J. (1985): Manual of clinical microbiology, American Society for Microbiology, Washington, DC. 4th ed: 918-919, 945.

Liu, R.-J., Yi, P.-J. (2013): Bacillus licheniformis and methods for detoxification of zearalenone, US 8404477 B2.

MacFaddin, J. F. (2000): Biochemical tests for identification of medical bacteria, 3rd ed. Lippincott Williams \& Wilkins, Philadelphia, PA. 
Meca, G., Ritieni, A., Zhou, T., Li, X.Z., Mañes, J. (2013): Degradation of the minor Fusarium mycotoxin beauvericin by intracellular enzymes of Saccharomyces cerevisiae, Food control, 33: 352-358.

Meca, G., Roig, M., Ferrer, E. and Mañes, J. (2014): Degradation of the bioactive compounds enniatins A, A1, B, B1 employing different strains of Bacillus subtilis, Journal of Food Processing and Technology, 5: 334.

Meca, G., Zhou, T., Li, X.Z., Ritieni, A., Mañes, J. (2013): Cyclohexadepsipeptide beauvericin degradation by different strains of Saccharomyces cerevisiae, Food and Chemical Toxicology, 59: 334-338.

O’Donnell, K., Kistler, H.C., Cigelnik, E., Ploetz, R.C. (1998): Multiple evolutionary origins of the fungus causing Panama disease of banana: concordant evidence from nuclear and mitochondrial gene genealogies, Proceedings of National Academy of Sciences, 95: 20442049.

Petchkongkaew, A., Taillandier, P., Gasaluck, P., Lebrihi, A. (2008): Isolation of Bacillus spp. from Thai fermented soybean (Thuanao): screening for aflatoxin B1 and ochratoxin A detoxification, Journal of Applied Microbiology, 104: 1495-1502.

Präve, P., Sukatsch, D., Vérte, L. (1972): Proticin, a new phosphorus-containing antibiotic. I Taxonomy, fermentation, isolation and biological properties, The Journal of Antibiotics, 25(1): 1-4.

Reddy, C. A. (ed.). (2007): Methods for general and molecular microbiology, 3rd ed. ASM Press, Washington, DC.

Riddell, R.W. (1950): Permanent stained mycological preparations obtained by slide culture, Mycologia, 42, 265-270.

Rodríguez, M.A., Cabrera, G., Gozzo, F.C., Eberlin, M.N., Godeas, A. (2011): Clonostachys rosea BAFC3874 as a Sclerotinia sclerotiorum antagonist: mechanisms involved and potential as a biocontrol agent, Journal of Applied Microbiology, 110(5): 1177-1186. 
Roig, M., Meca, G., Ferrer, E., Manes, J. (2013): Reduction of the enniatin A, A1, B, B1 by an in vitro degradation employing different strains of probiotic bacteria: Identification of degradation products by LC-MS-LIT, Toxicon, 70: 44-53.

Schroers, H.J. (2001): A monograph of Bionectria (Ascomycota, Hypocreales, Bionectriaceae) and its Clonostachys anamorphs, Studies in Mycology 46, 1-214.

Schroers, H.J., Samuels, G.J., Seifert, K.A. and Gams, W. (1999): Classification of the Mycoparasite Gliocladium roseum in Clonostachys as C. rosea, Its Relationship to Bionectria ochroleuca, and Notes on Other Gliocladium-like Fungi, Mycologia, 91 (2): 365-385.

SPSS: IBM Corp. Released 2013. IBM SPSS Statistics for Windows, Version 22.0. Armonk, NY: IBM Corp.

Sulkin, S. E. and Willett, J. E. (1940): A triple sugar-ferrous sulfate medium for use in identification of enteric organisms, Journal of Laboratory and Clinical Medicine, 25: 649-653.

Sun, X., He, X., Xue, K.S., Li, Y., Xu, D., Qian, H. (2014): Biological detoxification of zearalenone by Aspergillus niger strain FS10, Food and Chemical Toxicology, 72: 76-82.

Takahashi-Ando, N., Kimura, M., Kakeya, H., Osada, H., Yamaguchi, I. (2002): A novel lactonohydrolase responsible for the detoxification of zearalenone: enzyme purification and gene cloning, Biochemical Journal, 365 (1): 1-6.

Tan, H., Hu, Y., He, J., Wu, L., Liao, F., Luo, B., et al. (2014): Zearalenone degradation by two Pseudomonas strains from soil, Mycotoxin Research, 30: 191-196.

Toledo, A.V., Virla, E., Humber, R.A., Paradell, S.L., López Lastra, C.C. (2006): First record of Clonostachys rosea (Ascomycota: Hypocreales) as an entomopathogenic fungus of Oncometopia tucumana and Sonesimia grossa (Hemiptera: Cicadellidae) in Argentina, Journal of Invertebrate Pathology, 92: 7-10.

Turner, S., Pryer, K.M., Miao, V.P.W. and Palmer, J.D. (1999): Investigating deep phylogenetic relationships among cyanobacteria and plastids by small subunit rRNA sequence analysis, Journal of Eukaryotic Microbiology, 46: 327-338. 
White, T.J., Bruns, T., Lee, S., Taylor, J. (1990): Amplification and direct sequencing of fungal ribosomal RNA genes for phylogenetics. In: PCR Protocols: a guide to methods and applications, (Innis MA, Gelfand DH, Sninsky JJ, White TJ, eds), Academic Press, New York, USA: $315-322$.

Yi, P.-J., Pai, C.-K., Liu, J.-R. (2011): Isolation and characterization of a Bacillus licheniformis strain capable of degrading zearalenone, World journal of Microbiology and Biotechnology, 27(5): 1035-1043.

Zimbro, M.J., Power, D.A., Millwer, S.M., Wilson, G.E., Johnson, J.A. (2009): Difco and BBL manual: manual of biological culture media, Becton Dickinson and Co., Sparks, MD, 10th ed, 879-880. 


\section{Chapter 5}

\section{General discussion}

This study was conducted with the major objective of degrading enniatins (A, A1, B1 and B) into less toxic compounds by means of microorganisms (bacteria, yeast or fungi). The specific objectives consisted of purifying enniatins from fungal extract, then finding some microorganisms with the ability of breaking down enniatins into less toxic compounds and finally, identifying these active microorganisms. To achieve the first specific objective, we examined two Fusarium tricinctum strains for enniatins production on solid white bean medium, and then the best enniatins producing strain was used for the large scale production of enniatins on solid white bean medium using flash chromatography and recrystallization. During our study a short and efficient HPLC-DAD method was developed and validated for the detection of enniatins in the fungal extract. The results are comparable to mainly those of Wang et al. (2013) who optimized enniatin production using a F. tricinctum strain with the main focus on the type of medium used. According to these authors and the conditions applied during their study, the best harvesting times for enniatin production was between days 14 and day 18 when the production of enniatins A, A1, B and B1 peaked $1365 \mathrm{mg} / \mathrm{mL}$. During our study, the best harvest time for enniatin production was between day 24 and day 30. The enniatin B, B1 and B peaked at day 24 with $1228 \mathrm{mg} / \mathrm{L}$. Moreover, it was demonstrated, that $F$. tricinctum $\mathrm{O} 32$ produced about two times more enniatins than the strain DSM 23357. The slight observed differences could be due to the fermentation conditions that were different in both studies, but also to toxin variability and loss of toxin production by a Fusarium strain caused by mutations of the strain cultures stored for long period in fungal collections, as these may result in such differences (Moretti et al., 2007). After finding the best enniatin producer strain among the two tested ones, our aim was to purify a mixture of enniatins A, A1, B1 and B from the ethyl acetate crude extract using a combination of flash chromatography and recrystallization. Enniatins were confirmed using HPLC-MS and the purity was calculated after comparison with pure standards. The purified enniatin mixture contained about $77 \%$ of enniatin B, $17 \%$ of enniatin B1, $6 \%$ of enniatin A1 and $0.3 \%$ of enniatin A. The quantity of enniatins (A, A1, B, B1, B4, J1) purified from a solid corn medium fermented with $F$. tricinctum ITEM 9496 using a double purification with semipreparative LPLC and LC column process, produced quantities ranging from 30 to 300 
mg (Cuomo et al., 2013), whereas we obtained $3 \mathrm{~g}$ of enniatin mixture containing enniatin B, $\mathrm{B} 1$, A1 and A from $1 \mathrm{~kg}$ of white bean medium following flash chromatography and recrystallization. The combination of flash chromatography and recrystallization resulted in a higher yield of a mixture of enniatins A, A1, B1 and A compared to the data available in the literature using different fungal strains, extraction procedures, and purification techniques. No attempt was made to separate enniatin variants from each other, because during search for detoxifying microorganisms we wanted to have a scenario close to the natural conditions.

During last decades, much research has been conducted with the goal of decontaminating mycotoxin-contaminated food commodities and so as to reduce the health risk associated to high consumption of mycotoxins. Biodegradation is one of the techniques used for mycotoxin decontamination of feed or food; mycotoxins are removed under mild conditions, without harmful chemicals and significant losses in nutritive value and palatability of the decontaminated food and feed (Bata and Lásztity, 1999). Some examples of biological detoxification are reported in the literature. Enzymes isolated from the bacterium Sphingopyxis sp. MTA144 were demonstrated to detoxify fumonisin B1 (Heinl et al., 2010). The degradation of aflatoxin B1 by $F$. aurantiacum was studied by Line and Brackett (1995), it was found that aflatoxin degradation was independent of nutrients in the culture medium, suggesting a possibility of using this bacterium for aflatoxin detoxification in fermentation processes. Young et al., (2007) investigated the degradation of 12 trichothecenes by microorganisms isolated from chicken gut. Acethylation and deepoxydation were the two principal degradation pathways that were evidenced. The percentage of reduction detected ranged from $40-95 \%$.

The second part of this study involved the search of microorganisms able to degrade enniatins into less toxic compounds, we first isolated 114 bacteria and 34 fungi that were able to grow on media containing enniatins as the only carbon source. Then these strains were screened for enniatin degradation using HPLC. The results showed that two classes of microorganisms were isolated, one category which probably adsorb the mycotoxins and another category able to degrade the mycotoxin leading to the formation of new compounds. Microorganisms able to grow on medium containing the mycotoxin DON were proven to adsorb the mycotoxin instead of degrading the mycotoxin DON (Völkl et al., 2004). 1285 microbial cultures were tested and a mixed culture D107 was the only culture able to transform DON into new products mainly 3-keto-4-deoxynivalenol. No pure culture of the mixed culture D107 was 
able to consistently transform DON. In the present study, three pure cultures of two fungi and one bacterium were active regarding enniatin degradation. The culture supernatant of those microorganisms was analyzed using HPLC-MS. During our study, four enniatin degradation products were identified. Enniatin degradation products had protonated ions with $\mathrm{m} / \mathrm{z} 658$, 672, 686 and 700 for enniatin B, B1, A1 and A respectively, we assumed that these degradation products resulted from the hydrolysis of an ester (more likely) or an amide bond in the cyclic ring of enniatins. The degradation products obtained during the present study are different from those reported by Meca et al., (2014) who described two enniatins degradation products after the action of some bacillus strains on enniatins with loss of a hydroxyisovaleric acid residue in the structures of enniatin B and B1. Since the toxicity of enniatins is assumed to be derived from their ionophoric properties and from their action as uncouplers of oxidative phosphorylation (Shemyakin et al., 1969), enniatin degradation products demonstrated during the present study may be less toxic. Nevertheless, the degradation products must be purified and more studies must be made to test the toxicity of these compounds.

The taxonomy of the enniatins degrading microorganisms was performed and revealed their classification as Bacillus licheniformis, Clonostachys rosea and Acremonium strictum. This is the first report concerning the degradation of enniatins by the microbial cultures of Bacillus licheniformis, Clonostachys rosea and Acremonium strictum. Moreover this is the first report about enniatin degradation products generated from the hydrolysis of an ester bond (this is more likely because the hydrolysis of an ester bond requires less energy than that of an amide bond) as reported in the case of zearalenone. The enzyme zearalenone lactonohydrolase present in $C$. rosea cleaves the lactone rings from the backbone and transforms zearalenone to a far less potent compound (Kakeya et al., 2002). Although C. rosea does not degrade fumonisins, it was shown that it suppresses the synthesis of fumonisnins by $F$. verticillioides in co-cultures (Chatterjee et al., 2016). The ability of C. rosea to degrade enniatins as described in our study highlights the importance of $C$. rosea for mitigation of toxins in foodstuffs. We assumed that there is a hydrolase in the detoxifying microorganisms found in the present study capable of targeting the ester bond in the structure of enniatins.

B. licheniformis has also been demonstrated to degrade zearalenone in vitro, the idea of adsorption of the mycotoxin to the cell wall was excluded since an analysis of the bacterium cell wall was made after lysis (Yi et al., 2011). High capacity of secretion of the alkaline serine protease has made $B$. licheniformis one of the most important bacteria in industrial 
enzyme production (Schallmey et al., 2004). According to the evidence of bio-transformation of enniatins A, A1 and B1 demonstrated in this study, its potential use as a probiotic demonstrated previously and after further investigations concerning enterotoxins nonproduction by Bacillus licheniformis (Yi et al., 2011), the possibility of applying bacterium K4 as a food and feed supplement for bio-detoxification of enniatins should be considered.

Acremonium strictum which is not known to produce mycotoxins in food (Gams, 1971) is recognized for the production of compounds with biological activities such as cellulosic enzymes (Goldbeckt et al., 2013), acremostatin which exhibits a weak antibacterial activity against Micrococcus luteus, Salmonella typhimurium and Proteus vulgaris, as well as a moderate antioxidant activity leading to protection against oxidative stress induced cell death (Julianti et al., 2011). These findings suggest the use of $A$. strictum for the production of new enzymes with potential biotechnological applications.

Beauvericin, zearalenone and fumonisin detoxifying microorganisms Nocardia globulera (ATCC 55847) (Duvick and Rood, 2000), Rhodococcus erythropoli (ATCC 55851) (Duvick and Rood, 1998) and Exophiala spinifera (ATCC 74269) (Duvick* and Rood, 1998) originated from mouldy wheat, mouldy corn and field-grown kernels respectively. $B$. licheniformis and A. strictum were isolated from a soil sample originating from a wheat field, while C. rosea was isolated from Hazelnut sample. Since high enniatins concentration have been associated with cereal products such as wheat and with some nuts also, the hypothesis according which microorganisms present in the same habitat with some mycotoxins may possess or acquire through mutations the genes encoding for enzymes responsible for those mycotoxins degradation may be true.

More studies focusing on the purification of degradation compounds, the degradation mechanisms, the gene and enzymes involved in the mechanism must be carried out. Moreover a study of toxicity of the degradation products must be performed. 


\section{References}

Bata, Á. and Lásztity, R. (1999): Detoxification of mycotoxin-contaminated food and feed by microorganisms, Trends in Food Science and Technology, 10: 223-228.

Chatterjee, S., Kuang, Y., Splivallo, R., Chatterjee, P., and Karlovsky, P. (2016): Interactions among filamentous fungi Aspergillus niger, Fusarium verticillioides and Clonostachys rosea: fungal biomass, diversity of secreted metabolites and fumonisin production, BMC Microbiology, 16:83.

Cuomo, V., Randazzo, A., Meca, G., Moretti, A., Cascone, A., Eriksson, O., Novellino, E., Ritieni, A. (2013): Production of enniatin A, A1, B, B1, B4, J1 by Fusarium tricinctum in solid corn culture: structural analysis and effect on mitochondrial respiration, Food chemistry, 140: 784-793.

Duvick, J., Rood, T.A. (1998): Zearalenone detoxification composition and methods, US 5846812 A.

Duvick, J., Rood, T. A. (2000): Beauvericin detoxification methods using bacteria, US 6126934 A.

Duvick*, J., Rood, T., Wang, X. (1998): Fumonisin detoxification enzymes, US 5716820 A.

Gams, W. (1971): Cephalosporium-artige Schimmelpilze (Hyphomycetes), G. Fischer, Stuttgart, 1-262.

Goldbeck, R., Ramos, M.M., Pereira, G.A., Maugeri-Filho, F. (2013): Cellulase production from a new strain Acremonium strictum isolated from the Brazilian Biome using different substrates, Bioresource Technology, 128:797-803.

Heinl, S., Hartinger,D., Thamhesl, M., Vekiru, E., Krska, R. et al. (2010): Degradation of fumonisin B1 by the consecutive action of two bacterial enzymes, Journal of Biotechnology, 145: $120-129$.

Julianti, E., Oh, H., Jang, K.H., Lee, K.J., Lee, K.S., Oh, D.-C., Oh, K.-B. and Shin, J. (2011): Acremostrictin, a highly oxygenated metabolite from the Marine Fungus Acremonium strictum, Journal of Natural Products, 74 (12): 2592-2594. 
Kakeya, H., Takahashi-Ando, N., Kimura, M., Onose, R., Yamaguchi, I., Osada, H. (2002): Biotransformation of the mycotoxin zearalenone, to a non-oestrogenic compound by the fungal strain of Clonostachys sp. Bioscience, Biotechnology and Biochemistry, 66:27232726.

Line, J.E., Brackett, R.E. (1995): Role of toxin concentration and second carbon source in microbial transformation of aflatoxin B1 by Flavobacterium aurantiacum, Journal of Food Protection, 58:1042-1044.

Meca, G., Roig, M., Ferrer, E., Mañes, J. (2014): Degradation of the bioactive compounds enniatins A, A1, B, B1 employing different strains of Bacillus subtilis, Journal of Food Processing and Technology, 5:334.

Moretti, A., Mulè, G., Ritieni, A., Logrieco, A. (2007): Further data on the production of beauvericin, enniatins and fusaproliferin and toxicity to Artemia salina by Fusarium species of Gibberella fujikuroi species complex, International Journal of Food Microbiology, 118:158-163.

Schallmey, M., Singh, A., Ward, O.P. (2004): Developments in the use of Bacillus species for industrial production, Canadian Journal of Microbiology, 50(1): 1-17.

Shemyakin, M.M., Ovchinnikov, Y.A., Ivanov, N.T., Antonov, V.K., Vinogradova et al. (1969): Cyclodepsipeptide as chemical tools for studying ionic transport through membranes, Journal of Membrane Biology, 1: 402-430.

Völkl, A., Vogler, B., Schollenberger, M., Karlovsky, P. (2004): Microbial detoxification of mycotoxin deoxynivalenol, Journal of Basic Microbiology, 44(2): 147-56.

Wang, Z., Lin, X., Yu, M., Huang, Y., Qian, X., Shen, Y. (2011): Wine contamination by mycotoxin enniatin B from Fusarium tricinctum (Corda) Sacc., Journal of Food, Agriculture and Environment, 9: 182-185.

Yi, P.-J., Pai, C.-K., Liu, J.-R. (2011): Isolation and characterization of a Bacillus licheniformis strain capable of degrading zearalenone, World journal of Microbiology and Biotechnology, 27(5): 1035-1043.

Young, J.C., Zhou, T., Yu, H., Zhu, H., Gong, J. (2007): Degradation of trichothecene mycotoxins by chicken intestinal microbes, Food and chemical toxicology, 45: 136-143. 


\section{Summary}

Mycotoxins are secondary metabolites produced by fungi. Enniatins are a group of cyclohexadepsipeptides made of three hydroxyisovaleric acid molecules and alternating amino acids mostly valine, leucine and isoleucine. Enniatins have been associated with mainly cereal and cereal-based products, but also with nuts and some dried fruits. There is no evidence that high concentrations of enniatins in food commodities are not harmful for human and animal health in the long term. Therefore, methods to reduce enniatin content in food by transforming them into less toxic compounds are of crucial importance. Decontamination strategies for mycotoxin contaminated food or feed involve physical and chemical treatments which are to some extent successful but also pose some disadvantages like limited efficacy and possible losses of the sensorial and nutritive value. Therefore, intensive work has been done in the field of biodegradation of mycotoxins in food. Within that framework, the main objective of this study was to find microorganisms (bacteria, yeast or fungi) able to degrade enniatins into less toxic compounds. The strategy used involved a random search of such microorganisms followed by their classification.

Due to the high quantity of mycotoxins that are involved in such studies resulting in great expense, the first objective of this study was to purify enniatins from fungal extract. Therefore two strains of $F$. tricinctum (DSM 23357 and O32) were tested for enniatin production in solid white bean medium. Enniatins were extracted every 3 days. A short method for the detection and quantification of enniatins $\mathrm{A}, \mathrm{A} 1, \mathrm{~B}$ and $\mathrm{B} 1$ in the fungal crude extracts was established and validated using HPLC-DAD. For both studied strains, enniatin concentrations increased until 24 days with $614 \mathrm{~g} / \mathrm{kg}$ and $479 \mathrm{~g} / \mathrm{kg}$ for $F$. tricinctum O32 and DSM 23357 respectively. Throughout the 30 cultures days $F$. tricinctum O32 produced about two times more enniatins than the strain DSM 23357. As a result F. tricinctum $\mathrm{O} 32$ was used for the large scale production of enniatins over 30 days on solid white bean medium. Enniatin in the fungal crude extract were separated using flash chromatography and recrystallization. $3.18 \mathrm{~g}$ enniatins mixture/kg of white bean medium with a purity of $96.25 \%$ and containing about $77 \%$ enniatin B, $17 \%$ enniatin B1, $6 \%$ enniatin A1 and $0.3 \%$ enniatin A was obtained. 
The second part of this study involved the search for microorganisms having the ability to degrade enniatins. For this purpose, microorganisms were first isolated from several sources (grains, nuts, water, soil, cereal based-products, juice) based on their ability to grow on a minimal medium (MM) containing enniatin as the unique carbon source. Wash fluid from several materials were mixed with MM containing enniatins (final concentration $1 \mathrm{mg} / \mathrm{ml}$ ) and incubated for 7 days at $20^{\circ} \mathrm{C}$. The cultures were diluted 25-times in $\mathrm{MM}$ with enniatins (final concentration $1 \mathrm{mg} / \mathrm{ml}$ ) and incubated for further 21 days at $20^{\circ} \mathrm{C}$. In total 114 bacteria or yeast-like microorganisms and 34 fungi were isolated. The major sources of bacteria were water, grains and nuts from which 38, 35, 20 and 7 bacteria were isolated respectively. Whereas the majority of fungi originated from nuts (16), grains (10) and soil (3) samples. 20 bacteria and 10 fungi were not able to be cultivated and after a second selection using single culture in MM containing (final concentration $1 \mathrm{mg} / \mathrm{ml}$ ), 6 bacteria and 1 fungus did not grow anymore. Then 88 bacteria (and yeast-like microorganisms) and 23 fungi were screened for their property to break down enniatins into new compounds using HPLC-DAD (as described previously) in MM and in a minimal medium containing a limited amount of glucose and tryptone (MMGP). The results showed that enniatin concentrations were reduced in all the tested samples (enniatin A was mostly reduced than enniatin B). The HPLC-UV chromatograms of a bacterium (K4) and two fungi (F18 and F20) produced new signals compared to those of the controls. K4 and F18 originated from wheat field soil sample, whereas F20 was isolated form hazelnuts sample. These samples were further analyzed using LC-MS, differential metabolic profiling (Noise reduction, chromatogram alignment, normalization) and the mass of enniatins products were deduced and confirmed using extracted ions chromatograms and the corresponding mass spectra. Four enniatin products were identified using this technique; they were probably the products of hydrolysis of either an ester or an amide bond in the ring structure of enniatins, leading to the opening of the ring and therefore probably producing less toxic compounds. The molecular masses of the protonated ions of enniatins degradation products were 658, 672, 686 and 700 for enniatin $\mathrm{B}$, B1, A1 and A respectively.

Finally the identification of the pure cultures responsible for enniatin degradation was carried out using phenotypic (visual and microscopic), biochemical (for the bacterium only, gram test, catalase, sulfide-indole-motility, starch hydrolysis, citrate, urease tests...) and molecular characteristics of the microorganisms. The bacterium was preliminary identified as Bacillus sp. and the sequence of the 16S rRNA gene analysis enabled the species identification of the 
bacterium K4 as Bacillus licheniformis. Based on morphological characteristics (visual and microscopic observations, spore size, conidia, conidiophores, presence or absence of septae...), fungi F18 and F20 were identified as Acremonium sp. and Clonostachys sp. respectively. The sequence of the ITS1, 5.8S RNA subunit and ITS2 of the DNA revealed that F18 and F20 were Acremonium strictum and Clonostachys rosea. 


\section{Acknowledgements}

I would like to sincerely thank my supervisor Prof. Dr. Petr Karlovsky for having confidence in me and giving me the opportunity to work in his group, for providing me with this interesting subject. I thank him for giving me the scientific guidance and motivation I required. I am grateful for the experience I have gained through working in his group during the last three years.

I acknowledge Prof. Dr. Tapani Yli-Mattila and PD Dr. Franz Hadacek as members of my $\mathrm{PhD}$ defense committee.

I would like to express my deepest thank to Dr. Katharina Pfohl for introducing me the HPLC and LC-MS equipment, for her advice in the case of trouble-shooting and also for her advice, tips or suggestions concerning laboratory experiments throughout my laboratory work. My gratitude goes to Dr. Kirstin Feussner (Department of Plant Biochemistry, Albrecht-vonHaller Institute for Plant Sciences, Georg-August-University, Göttingen) who carried out the UPLC analysis for identification of enniatins degradation products used for a presentation at the $38^{\text {th }}$ mycotoxin workshop.

My gratitude goes to Ruth Pilot and Heike Rollwage for their advice, constant and prompt technical support. I thank Ruth Pilot particularly for her involvement and collaboration during the molecular identification of enniatin detoxifying microorganisms. Apart from this, I thank Ruth Pilot and Heike Rollwage very much for checking my thesis.

I am thankful to Dr. Athanassios Mavridis for providing me some bacteria strains used as positive or negative controls for the bacteria taxonomy and Evelin Vorbeck for her assistance in using the light microscope in the division of general plant pathology and crop protection of Georg-August-University Göttingen.

My gratitude goes to all the administrative staff of molecular phytopathology and mycotoxin research department for a good collaboration and support, especially to Mr. Thomas Oesterreich, Mr. Siegfried Opolka and Mrs. Evelin Kistner. 
I am very grateful to the apprentices Christian Kolodziel, Britta Engelhardt, Jonas Waterott for their technical assistance. I had a great honor to share the time with our PhD students: Ruiyu Zhu who encouraged me a lot during the first months, Yi Kuang who introduced me into the use of the flash chromatography device, Raana Dastjerdi, Mohammed Sherif, Olaf Henke, Pervin Akter, Rasoul Abousaeedi, Riyan Anggriawan, Mohammad Alhussein, Albatol Alsarrag, Vincent Ayugi, Zana Jamal Kareem, Yang Xu, Ling Su, Maria Vinas for the nice environment and collaboration in the same office, for the delicious international specialties and conversations about other cultures, habits and norms we used to share. I have learned a lot from you and you all made my moments more enjoyable during my $\mathrm{PhD}$.

I am thankful to my family in Cameroon and in Germany for the diverse support, love and motivation they have given me during the last years and my friends in Germany and abroad for scientific discussions but also for all the fun, "extra $\mathrm{PhD}$ " discussions and leisure we shared together.

My deepest thank goes to my husband Andreas for being at my side.

Thank you! 


\section{Curriculum vitae}

\section{Personal information}

Name

E-Mail

\section{Work experience}

$10 / 2011-03 / 2012$

08/2006-08/2008

01/2003-12/2003

\section{Academic education}

$02 / 2013$ to date

$10 / 2008-10 / 2011$

10/2003-10/2006

10/1999-10/2003
Rosine Ghislaine Suchfort (born Fotso)

r.fotso@stud.uni-goettingen.de
Research assistant at University of Hohenheim in Stuttgart, Institut für Lebensmittelwissenschaft und Biotechnologie, Lebensmittel Tierischer Herkunft (150e).

Research assistant and scientific staff at University of Dschang in Cameroon, Nutrition and food chemistry.

Laboratory assistant in the production department at CAMLAIT (Cameroonian Society of Dairy Products) in Yaoundé (Cameroon).
International $\mathrm{PhD}$ Program for Agricultural Sciences in Göttingen (IPAG) at Georg-August-University Göttingen, Germany.

Master program „Organic Food Chain Management“, Faculty of Agricultural Sciences, University of Hohenheim (Stuttgart, Germany). Main emphasis: Food chemistry. Final degree: M.Sc Organic Food Chain Management (new name: Organic Agriculture and Food systems).

Master program Biochemistry, Faculty of natural Sciences, University of Dschang (Cameroon). Main emphasis: Nutrition, food science and quality. Final degree: M.Sc. Biochemistry.

Bachelor Biochemie, Faculty of natural Sciences, University of Dschang (Cameroon). Final degree: B.Sc. Biochemistry. 


\section{Publications}

Suchfort, R. G., Karlovsky, P. (2016): Degradation of enniatins by Clonostachys rosea, Acremonium strictum and Bacillus licheniformis, $38^{\text {th }}$ Mycotoxin workshop Berlin 2016, Lecture, p 39.

Suchfort, R. G., Karlovsky, P. (2016): Purification of enniatins from F. tricinctum cultures in solid white bean medium, $38^{\text {th }}$ Mycotoxin workshop Berlin 2016, Poster, p 137.

Teguia, A., Telefo, P. B., Fotso, R. G. (2007): Growth performances, organ development and blood parameters of rats fed graded levels of steeped and cooked taro tuber (Colocasia esculenta var. esculenta) meal, Livestock Research for Rural Development, 19(6): 76.

Fotso, R.G., Teguia, A., Telefo, P.B. (2006): Effects of some processing methods on oxalate content and nutrient composition of taro tuber (Colocasia esculenta var esculenta) flour as a potential energy source for animal feeding Bulletin of Animal Health and Production in Africa, 54(4): 75-78. 


\section{Declarations}

1. I, hereby, declare that this Ph.D. dissertation has not been presented to any other examining body either in its present or a similar form.

Furthermore, I also affirm that I have not applied for a Ph.D. at any other higher school of education.

Gottingen,

2. I, hereby, solemnly declare that this dissertation was undertaken independently and without any unauthorized aid.

Gottingen, 